University of Louisville

ThinkIR: The University of Louisville's Institutional Repository

Electronic Theses and Dissertations

8-2008

\title{
Computational analysis of expressed sequence tags for understanding gene regulation.
}

\author{
I. Elizabeth Cha 1973- \\ University of Louisville
}

Follow this and additional works at: https://ir.library.louisville.edu/etd

\section{Recommended Citation}

Cha, I. Elizabeth 1973-, "Computational analysis of expressed sequence tags for understanding gene regulation." (2008). Electronic Theses and Dissertations. Paper 227.

https://doi.org/10.18297/etd/227

This Doctoral Dissertation is brought to you for free and open access by ThinkIR: The University of Louisville's Institutional Repository. It has been accepted for inclusion in Electronic Theses and Dissertations by an authorized administrator of ThinkIR: The University of Louisville's Institutional Repository. This title appears here courtesy of the author, who has retained all other copyrights. For more information, please contact thinkir@louisville.edu. 


\title{
COMPUTATIONAL ANALYSIS OF EXPRESSED SEQUENCE TAGS FOR UNDERSTANDING GENE REGULATION
}

\author{
By \\ I. Elizabeth Cha \\ B.S., Chemistry, Chung Yuan Christian University, Taiwan, R.O.C., 1995 \\ M.S., Computer Engineering and Computer Science, University of Louisville, 2002
}

\begin{abstract}
A Dissertation
Submitted to the Faculty of the

Graduate School of the University of Louisville in Partial Fulfillment of the Requirements

for the Degree of
\end{abstract}

Doctor of Philosophy

Department of Computer Engineering and Computer Science

University of Louisville

Louisville, Kentucky

August 2008 
Copyright 2008 by I. Elizabeth Cha

All rights reserved 


\title{
COMPUTATIONAL ANALYSIS OF EXPRESSED SEQUENCE TAGS FOR UNDERSTANDING GENE REGULATION
}

\author{
By \\ I. Elizabeth Cha \\ B.S., Chemistry, Chung Yuan Christian University, Taiwan, R.O.C., 1995 \\ M.S., Computer Engineering and Computer Science, University of Louisville, 2002 \\ A Dissertation Approved on
}

July 30,2008

by the following Dissertation Committee:

Dr. Eric C. Rouchka, CECS Department, Dissertation Director

Dr. Dar-jen Chang, CECS Department

Dr. Nigel G. F. Cooper, ASNB Department

Dr. Adel S. Elmaghraby, CECS Department

Dr. Ming Ouyang, CECS Department 


\section{DEDICATION}

This dissertation is dedicated to my parents

Mr. Shihgene Cha

and

Mrs. Hsiujen Chen Cha

who have given me invaluable educational opportunities and unconditional love. 


\section{ACKNOWLEDGEMENTS}

It is a pleasure to finish this dissertation because it is proof of the work that I have done these past few years during my doctoral training. For this I would like to thank my advisor Dr. Eric C. Rouchka. His knowledge in Bioinformatics is so comprehensive that he has answered many of my questions and corrected my misunderstanding in the field. His patience in research has served as a testimony for me on how to be a researcher in the future. Words of encouragement always came at the right time to push me to continue working on my research. He really knew the right timing to needle me about moving on in my research!!!

I would like to thank Dr. Dar-jen Chang, who helped me to survive the first semester in Computer Science when I returned to school for my master's in the Fall of 2000. His breadth of research has taken me into different fields of Computer Science.

I also appreciate Dr. Adel S. Elmaghraby, the department chair for Computer Engineering and Computer Science (CECS) and my mentor in the future professor program from Fall 2003 to Spring 2004. His broad knowledge in Computer Science opened my eyes to a different world. Without his support, I would not have been able to enter the Computer Science and Engineering (CSE) program as a substitute for a Speed school scholarship fellow who did not receive a visa to study in United States in Spring 2003. This allowed me to enter the field of research and opened the possibility of becoming a professor rather than just a teacher in my future career. 
I would like to thank Dr. Nigel G. F. Cooper for his financial support from Kentucky Biomedical Research Infrastructure Network (KBRIN). His support enabled me to concentrate on my research without worrying about money after my school fellowship ended in 2004. He also provided access to his laboratory so I was able to observe and participate research.

I would like to thank Dr. Ming Ouyang for being on my committee. Although he joined the CECS department recently, he came in at the perfect timing to participate in the committee and helped me with his experience and knowledge.

I am thankful for Christopher Whitaker and Xiaohong Li for their support and work in the wet lab to finish the evaluation for the computational prediction in alternative splicing events in rat PDE4 sequences. In addition, I appreciate Stephanie Dearing, Michelle Padgett, and Bethany Coates for their work in KRBIN. Moreover, Nate Johnson and Ed Birchler for their assistance with the technical service of KyBRIN cluster, Ron Lile, who named Patience as my middle name, and his technical assistants from the CECS department for computer support, Tim and Amy Hardin for their friendship, Christy Bogard, Joe Morris and Xiaohui Cui from the Bioinformatics Research Laboratory for their research assistance and friendships, Carlos Rojas for his help in programming and his friendship.

I am deeply grateful to my uncle Stanley Jsa and my aunt Shoutong Jsa who have greatly supported me since I came to Louisville. Their unconditional love and financial support have helped me through both my master's and Ph.D. Although I am far away from home, Stanley and Shoutong always open their door for me so I can feel like I'm at home. In addition, Uncle Stan was the one who encouraged me to study abroad and 
actually applied for me to attend the graduate school at the University of Louisville. Without his push, I would not be here today.

There are many people to whom I would also like to extend thanks: Sarah Cecil, Amy Filippini, Karen Whitlock, Melanie Helfrich, Jessica Ferris, Selina Jones, Megan Jacobs, Rich Gimmel, Juan and Vickiee Villafãne, Jan Howell, Sarah Oliver, Katherine Nally, Kathleen Clarkson, Marty Thomas, Greg McCarty, Elizabeth Nicholson, Mark Walden, Ward and Anita Mowery, Clair and Patti Morgan, Frances Jones, Rich and Brittani Hoyer, Pauline Chiu, Rhonda Graham, friends from Southeast Christian Church and Lyndon Christian Church, and family for their encouragement and prayers. I thank the Card Talk members in the Toastmasters Club International University of Louisville Chapter for helping me practice my speech and presentation. I am also grateful for Sandy Chiu and Matt Bader, Bin Cao and Jun $\mathrm{Wu}$ for their friendships since I moved to Louisville, Kentucky. I am especially thankful for Mary Ann Nestmann, for the time she took to review my "Incomplete English" writing. Her comments and suggestions helped me to express my work in standard English grammar.

In addition, I would like to thank Candace "Weiwei" Huang and Michael Lee and their son Anson. Weiwei especially shared all my happiness, sadness, and discomfort during the close-to-six-year-long doctoral training. She spent a lot of time listening to my complaining and crying about many things, from school work to personal situation. The Lee family opened their home and family to me so I would have a place to visit during the semester breaks and enjoy being an instant aunt for their son Anson.

Finally, I want to thank my parents, Shihgene and Hsiujen Cha. Their prayers and support kept me going in my research and study. Without their love and dedication, I 
would not have made it. Although we have to be apart during my study in the United States, they spent time and money so we could stay connected by email and phone. I also thank my brother Samuel for his support and encouragement. Sam is working on his Ph.D. in history at National Taiwan University and currently serves in the military in order to fulfill his citizenship requirement. Sam, although you entered the Ph.D. program earlier than me, since you had to stop temporarily for the military service, I am sorry, but I will graduate earlier.

One last person I need to mention is Dr. Gwong-chien Sun. He was the one who suggested that I apply for the CSE Ph.D. program in the CECS department at the University of Louisville instead of going to another university when I could not find a job and struggled with whether to stay in the U.S. or go home after I received my master's degree. Regrettably, he was not able to see me finish my Ph.D. since he passed away in March, just over two months after I started the doctoral training in Spring 2003.

The research projects in current BLAST software comparison, computational predictions of alternative splicing patterns in rat PDE4 sequences and pseudogene detections and characterization were supported by the NIH-NCRR Grant P20RR16481 (Nigel G. F. Cooper, PI) and NIH-NIEHS Grant P30ES014443 (Kenneth S. Ramos, PI). I will quote a few verses from the English Standard Version of the Bible as an acknowledgement to the One who has enabled me to stay in the $\mathrm{Ph} . \mathrm{D}$. program.

“... He will surely be gracious to you at the sound of your cry. As soon as He hears it, He answers you. And though the Lord give you the bread of adversity and the water of affliction, yet your Teacher will not hide Himself anymore, but your eyes shall 
see your Teacher. And your ears shall hear a word behind you, saying "This is the way, walk in it," when you turn to the right or when you turn to the left.” Isaiah 30:19b 21 Today is July $4^{\text {th }}, 2008$ and I have finished writing the acknowledgement of my dissertation in Louisville, Kentucky, United States of America. 


\section{ABSTRACT \\ COMPUTATIONAL ANALYSIS OF EXPRESSED SEQUENCE TAGS \\ FOR UNDERSTANDING GENE REGULATION \\ I. Elizabeth Cha}

July 30,2008

High-throughput sequencing has provided a myriad of genetic data for thousands of organisms. Computational analysis of one data type, expressed sequence tags (ESTs) yields insight into gene expression, alternative splicing, tissue specificity gene functionality and the detection and differentiation of pseudogenes. Two computational methods have been developed to analyze alternative splicing events and to detect and characterize pseudogenes using ESTs.

A case study of rat phosphodiesterase 4 (PDE4) genes yielded more than twentyfive previously unreported isoforms. These were experimentally verified through wet lab collaboration and found to be tissue specific. In addition, thirteen cytochrome-like gene and pseudogene sequences from the human genome were analyzed for pseudogene properties. Of the thirteen sequences, one was identified as the actual cytochrome gene, two were found to be non-cytochrome-related sequences, and eight were determined to be pseudogenes. The remaining two sequences were identified to be duplicates.

As a precursor to applying the two new methods, the efficiency of three BLAST algorithms (NCBI BLAST, WU BLAST and mpiBLAST) were examined for comparing 
large numbers of short sequences (ESTs) to fewer large sequences (genomic regions). In general, WU BLAST was found to be the most efficient sequence comparison tool.

These approaches illustrate the power of ESTs in understanding gene expression. Efficient computational analysis of ESTs (such as the two tools described) will be vital to understanding the complexity of gene expression as more high-throughput EST data is made available via advances in molecular sequencing technologies, such as the current next-generation approaches. 


\section{TABLE OF CONTENTS}

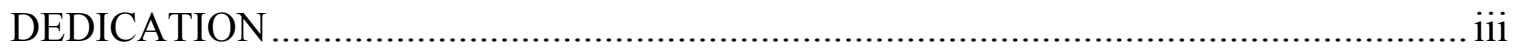

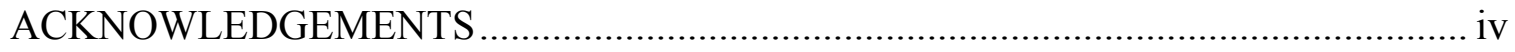

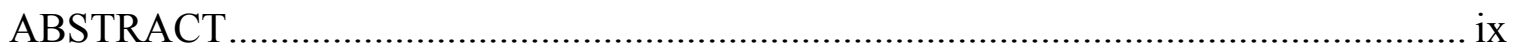

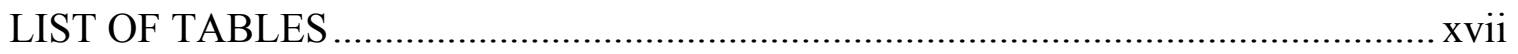

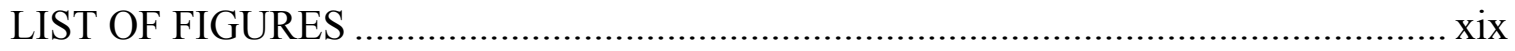

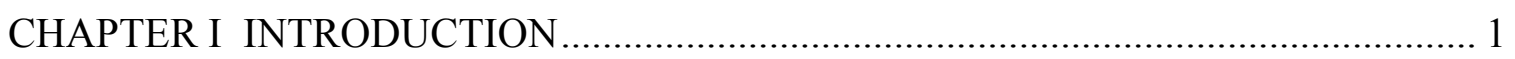

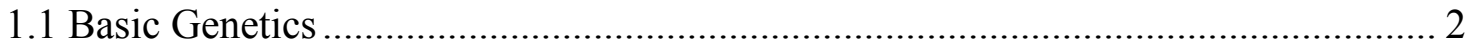

1.2 DNA

1.3 RNA …

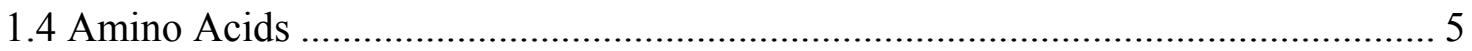

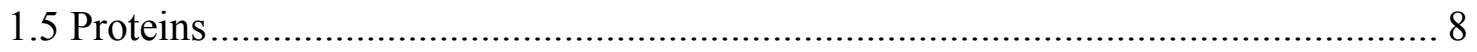

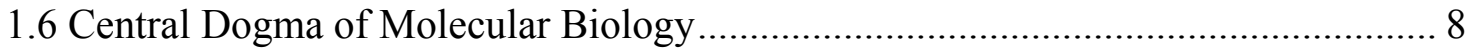

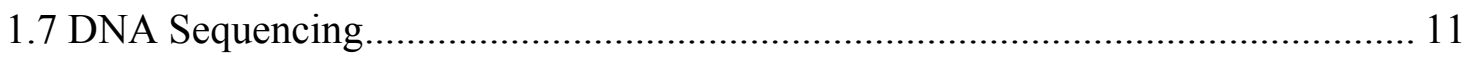

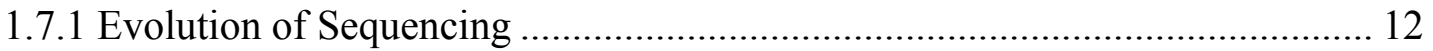

1.7.2 Expressed Sequence Tags...................................................................... 15

1.7.3 Human Genome Project (HGP) ................................................................... 16

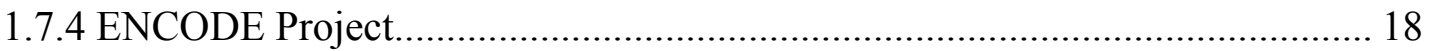

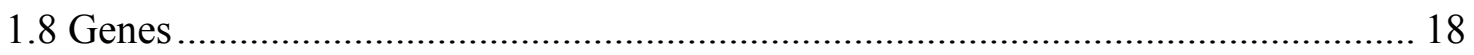

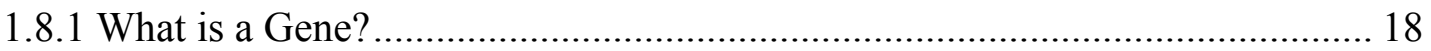

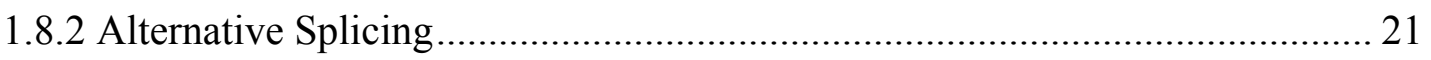




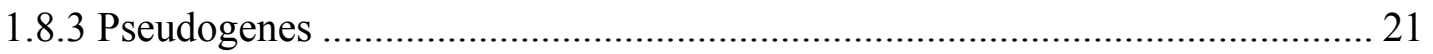

1.9 Bioinformatics and Computational Biology …………..................................... 22

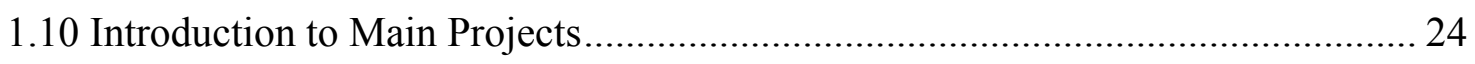

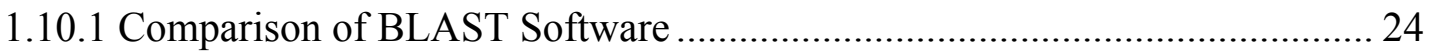

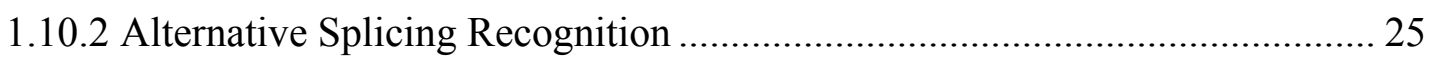

1.10.3 Pseudogene Detection and Characterization ................................................ 26

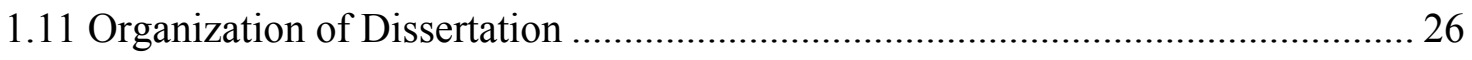

CHAPTER II DATA SOURCES AND DATA PREPROCESSING ............................... 28

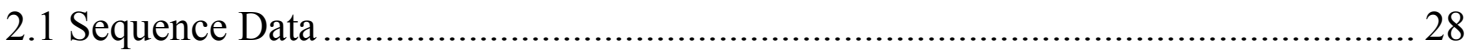

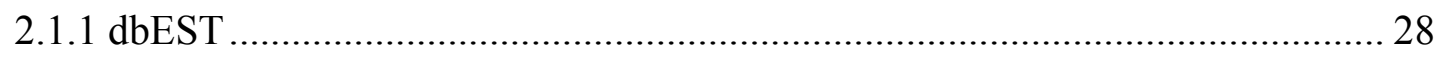

2.1.2 Reference Sequence (RefSeq) ……............................................................ 30

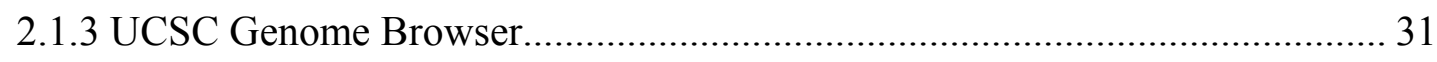

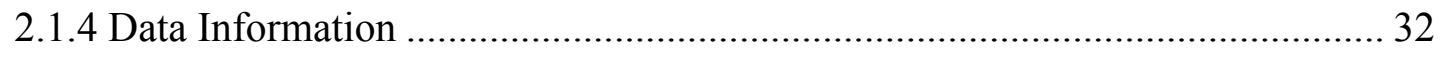

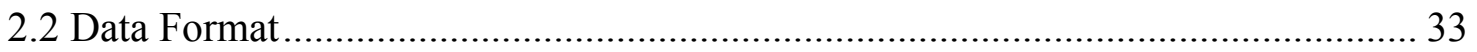

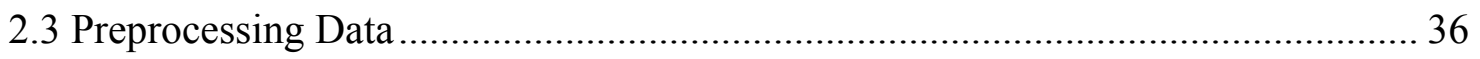

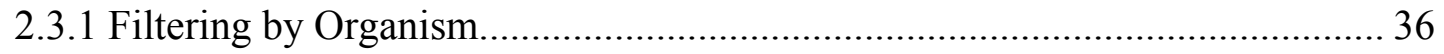

2.3.2 Masking Repetitive Elements...................................................................... 37

2.3.3 Filtering Low Complexity Regions .............................................................. 37

CHAPTER III COMPUTATIONAL SEQUENCE ALIGNMENT .................................. 39

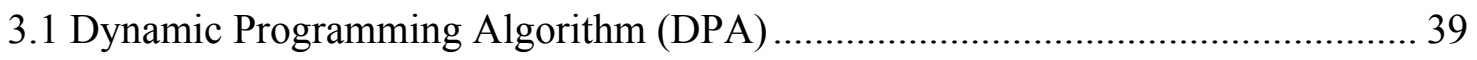

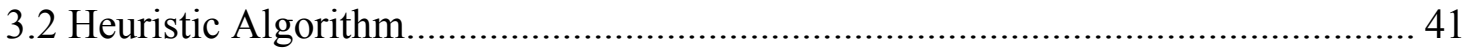

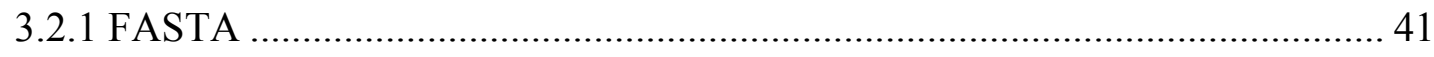

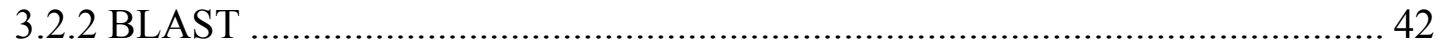




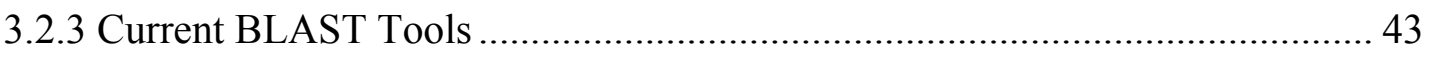

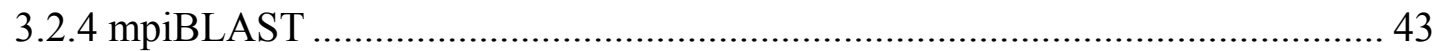

3.3 EST to Genome Alignment Algorithms ................................................................. 44

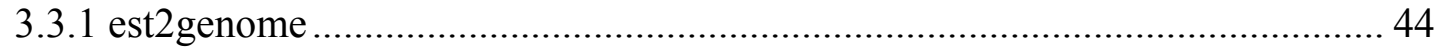

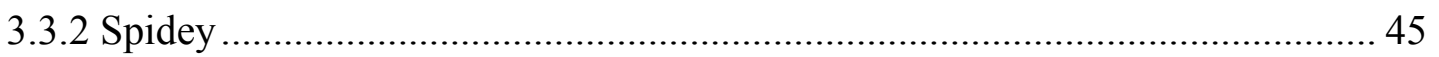

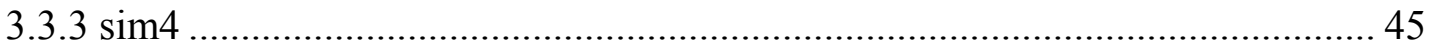

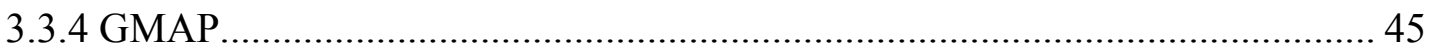

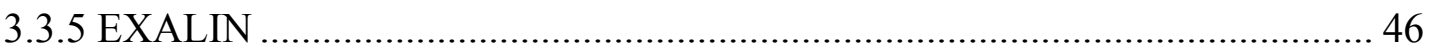

3.4 Case Study in Comparison of Current BLAST Software ……………………...... 46

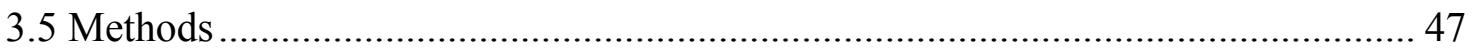

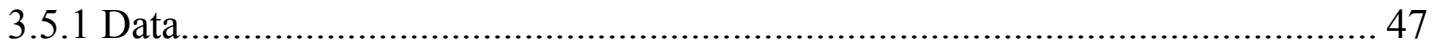

3.5.2 Types of Sequence Searches ...................................................................... 50

3.5.3 System and Software .................................................................................. 51

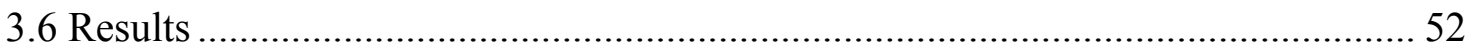

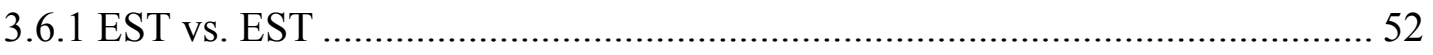

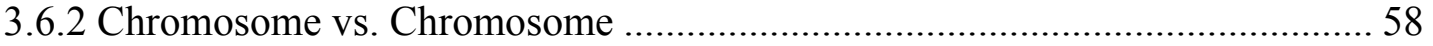

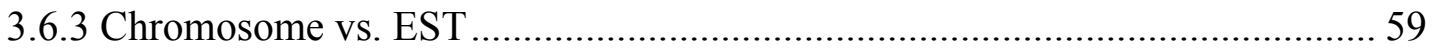

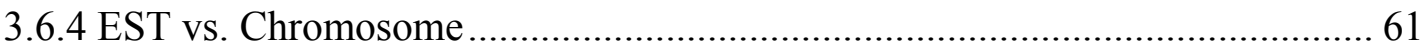

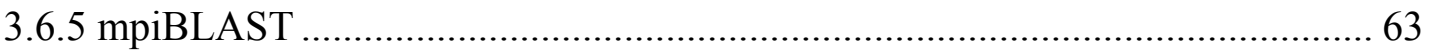

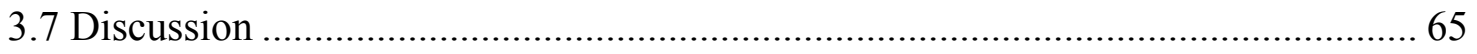

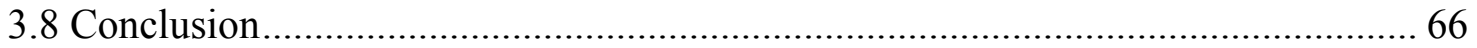

CHAPTER IV COMPUTATIONAL METHOD FOR ALTERNATIVE SPLICE

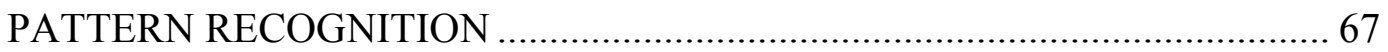




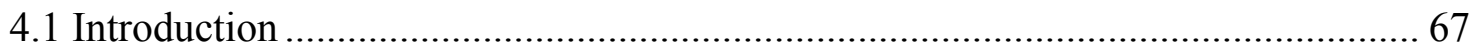

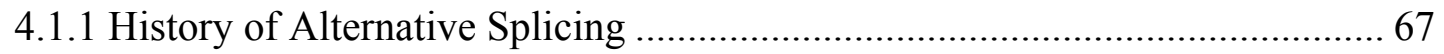

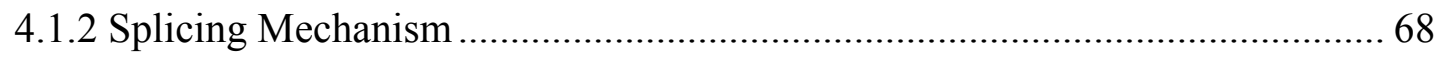

4.1.3 Alternative Splicing Modes ........................................................................... 70

4.1.4 Characteristics of Alternative Splicing........................................................ 72

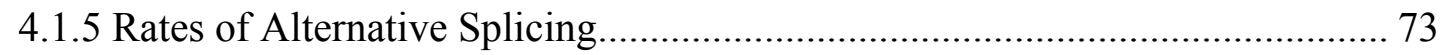

4.2 Research in Alternative Splicing Detection .......................................................... 73

4.2.1 Automatic Methods for Detecting Alternative Splicing.................................. 73

4.2.2 Research of Automatic Applications in Alternative Splicing Detection.......... 74

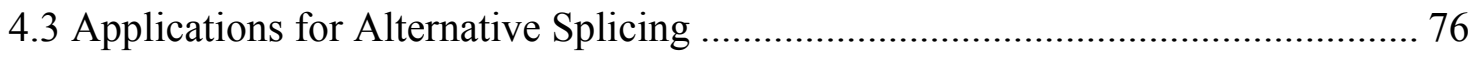

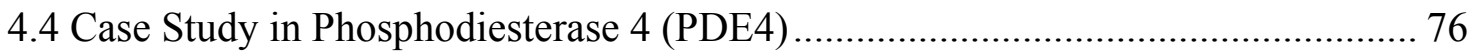

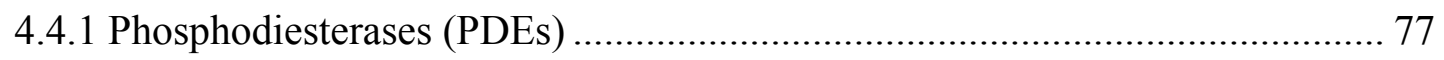

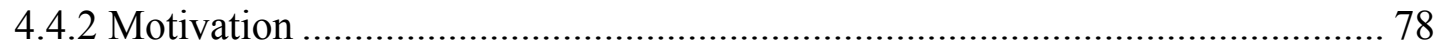

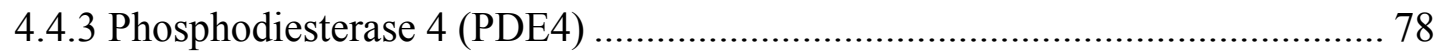

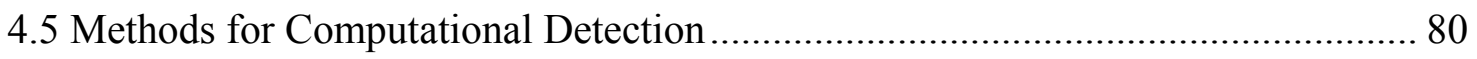

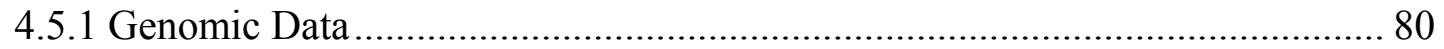

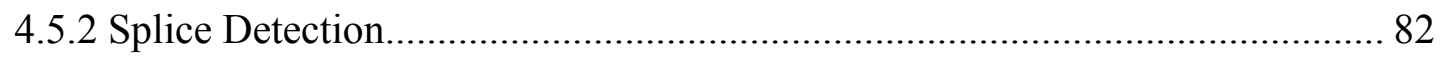

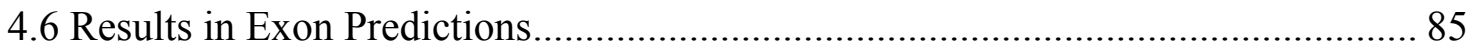

4.6.1 Spliced Pattern Observation ..................................................................... 90

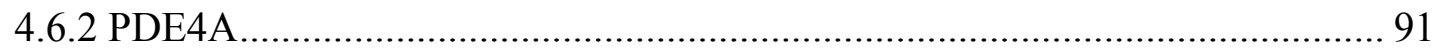

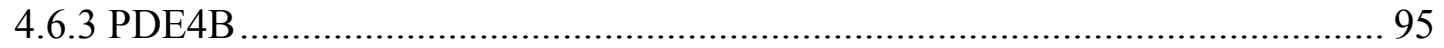

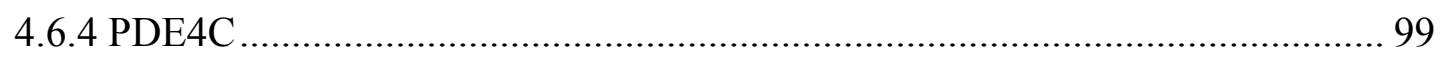

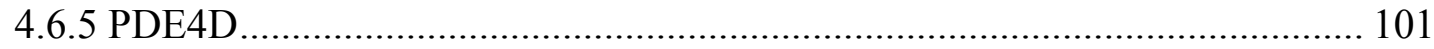




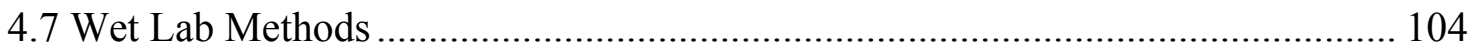

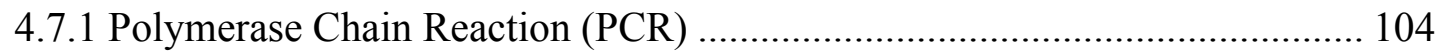

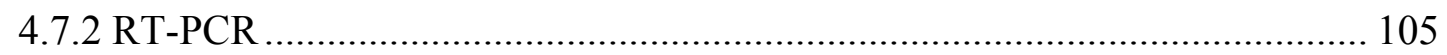

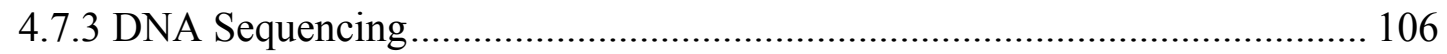

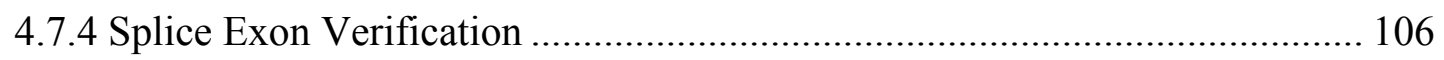

4.8 Results in Verified Predicted Exons................................................................... 107

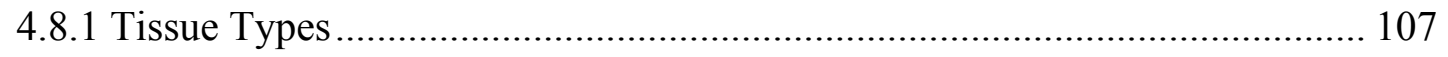

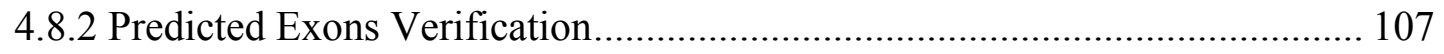

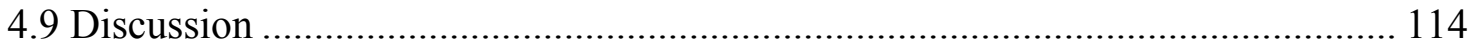

CHAPTER V COMPUTATIONAL METHOD FOR PSEUDOGENE DETECTION

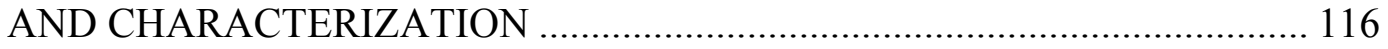

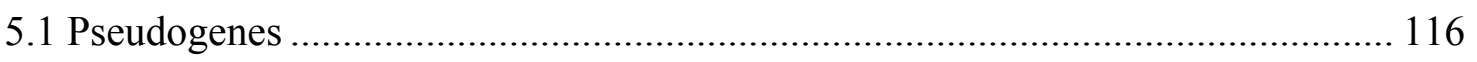

5.1.1 Processed Pseudogenes ............................................................................ 117

5.1.2 Unprocessed Pseudogenes.......................................................................... 118

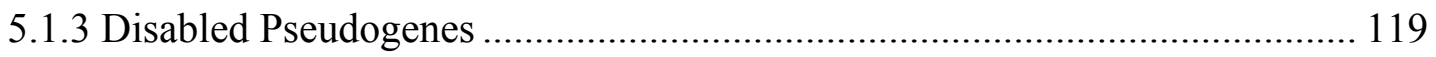

5.1.4 Functional Pseudogenes ............................................................................... 119

5.1.5 Motivations for Pseudogene Detection and Characterization ........................ 120

5.2 Current Automatic Pseudogene Detecting Methods ........................................... 120

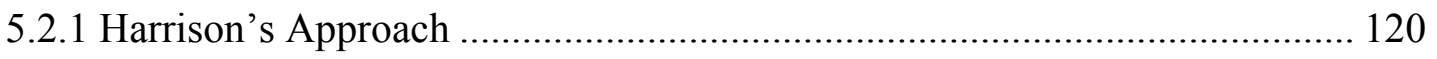

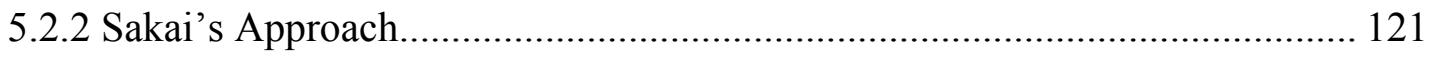

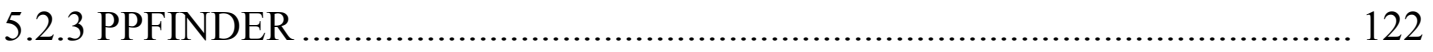

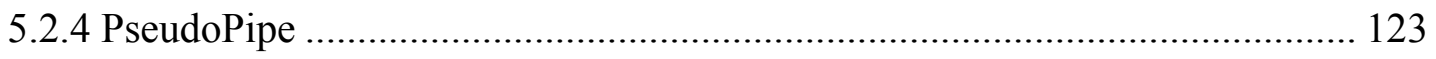

5.2.5 Pseudogenes Analysis in ENCODE Project.................................................... 124 
5.3 Characteristics for Pseudogene Detection.......................................................... 125

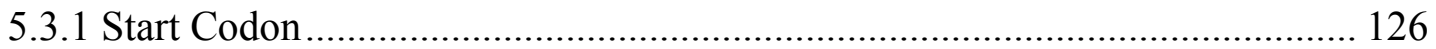

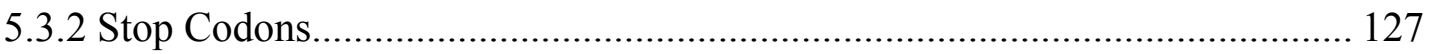

5.3.3 Poly-A Signals and Poly-A Tails ............................................................... 128

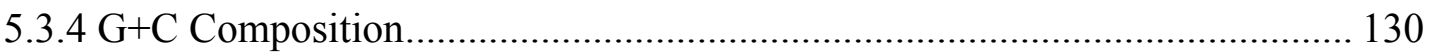

5.3.5 Similar, Synonymous vs. Nonsynonymous Mutations................................... 130

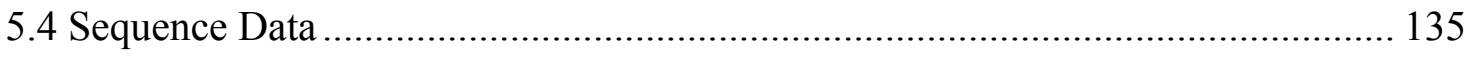

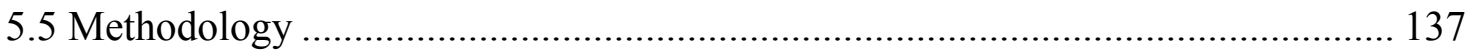

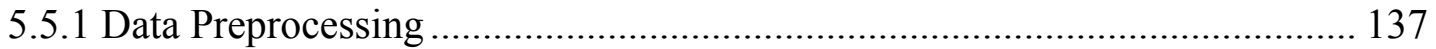

5.5.2 ESTs to Genome Alignment.......................................................................... 137

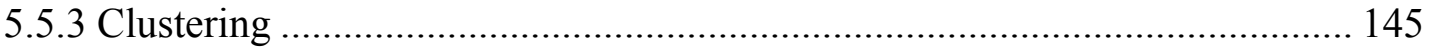

5.5.4 Pseudogene Detection and Characterization ................................................. 146

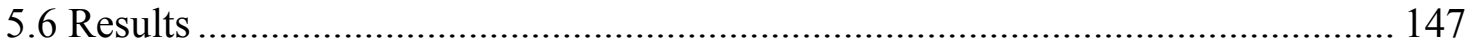

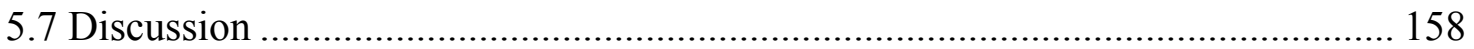

CHAPTER VI CONCLUSIONS AND DIRECTIONS FOR FUTURE RESEARCH .. 161

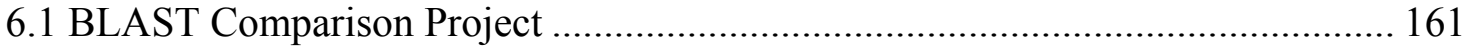

6.2 Rat Phosphodiesterase 4 (PDE4) Project ........................................................... 162

6.3 Computational Method of Detecting Alternative Splicing Events....................... 163

6.4 Computational Method for Pseudogene Detection and Characterization ............. 163

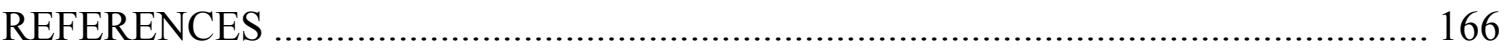

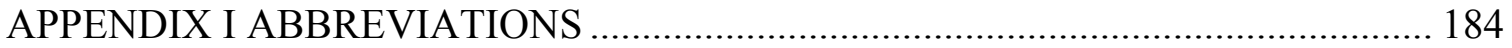

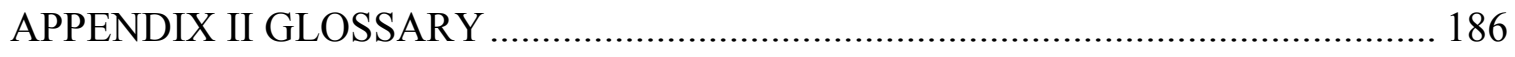

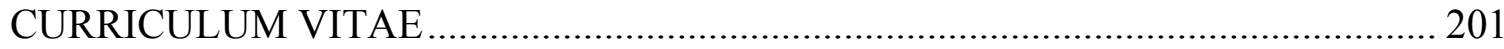




\section{LIST OF TABLES}

Table 1.1: IUPAC-IUB Nucleic Acid and Amino Acid Codes ....................................... 3

Table 1.2: The Genetic Code (RNA to Amino Acids)................................................... 6

Table 1.3: Structure and Classification of the 20 Common Amino Acids ....................... 7

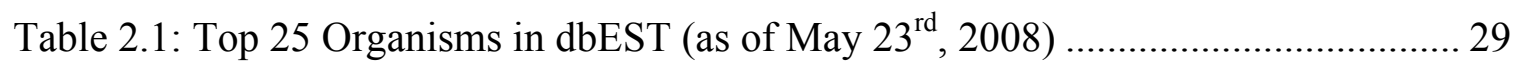

Table 2.2: Statistical Information of RefSeq Sequences (as of May $\left.9^{\text {th }}, 2008\right) \ldots \ldots \ldots \ldots . . . . .31$

Table 2.3: Available Sequence and Annotation Downloads in UCSC Genome Browser 32

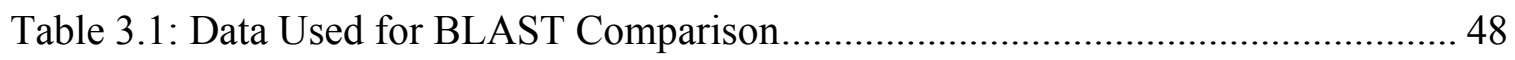

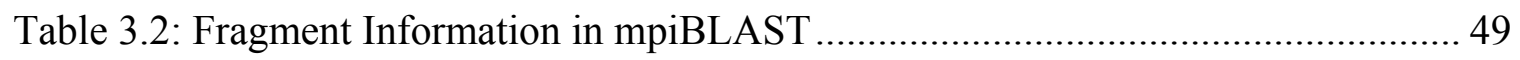

Table 3.3 ncbiBLAST Performs Best when the Number of Sequences in the Database is

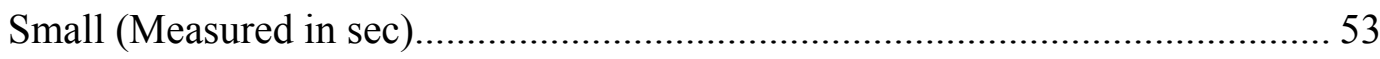

Table 3.4: wuBLAST Performs Best when the Number of Sequences in the Databases is

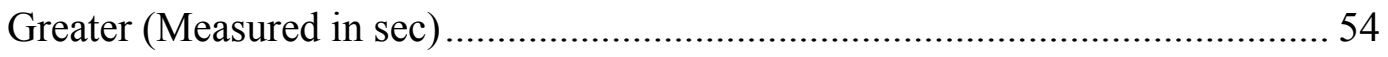

Table 4.1: Rat PDE4 Family mRNA Sequence Information...................................... 81

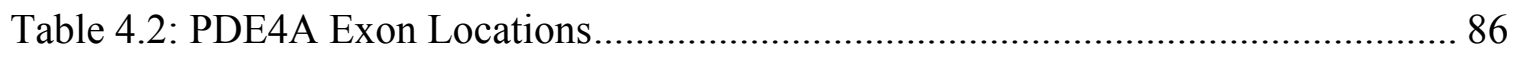

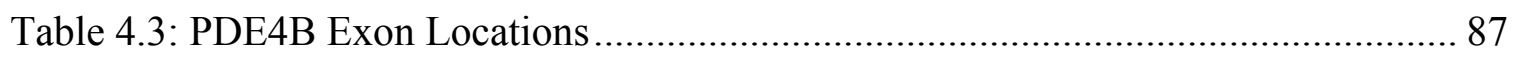

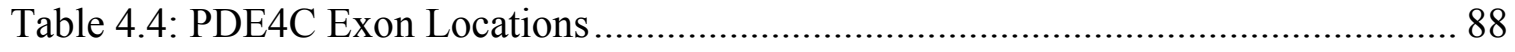

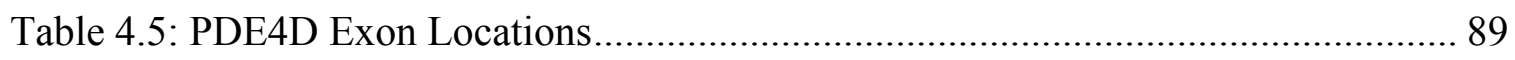

Table 4.6: Single Exon Splicing Patterns and Tissue Types for PDE4A ....................... 92

Table 4.7: Multiple Exon Splicing Patterns and Tissue Types for PDE4A.................... 93

Table 4.8: Single Exon Splicing Patterns and Tissue Types for PDE4B........................ 96 
Table 4.9: Multiple Exon Splicing Patterns and Tissue Types for PDE4B .................... 97

Table 4.10: Splicing Patterns and Tissue Types for PDE4C …................................ 100

Table 4.11: Splicing Patterns and Tissue Types for PDE4D ..................................... 102

Table 4.12: PCR Primer Pairs for Selected Predicted Exons.................................... 108

Table 4.13: Sequencing Results (Primer 14-1 in PDE4A) ......................................... 111

Table 4.14: Sequencing Results (Primer 15-1 in PDE4B).......................................... 112

Table 4.15: Sequencing Results (Primer 23-1 in PDE4B)........................................ 113

Table 5.1: Start Codon Alternative Patterns and Their Scores ................................... 127

Table 5.2: Stop Codon Alternative Patterns and Their Scores ................................... 128

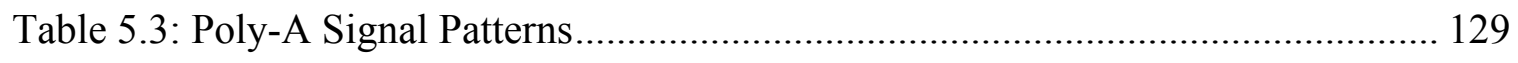

Table 5.4: The Genetic Code (RNA to Amino Acids).............................................. 132

Table 5.5: Percentage of $\mathrm{N}$ and $\mathrm{G}+\mathrm{C}$ Composition in Sequences ............................... 148

Table 5.6: Frequency of Start Codon Patterns ........................................................ 149

Table 5.7: Frequency of Stop Codon Patterns ................................................... 150

Table 5.7: Frequency of Stop Codon Patterns (Continued) ....................................... 151

Table 5.8: Frequency of Poly-A Signal Patterns ................................................. 152

Table 5.9: Poly-A Site Information for Each Sequence ......................................... 153

Table 5.10: Counts of Similar Synonymous Mutations, Synonymous Mutations, Nonsynonymous Mutations, and Insertion for Each Sequence ....................... 155

Table 5.11: Score for Pseudogene Candidate Sequences ........................................ 157 


\section{LIST OF FIGURES}

Figure 1.1: A diagram of a sugar-ring molecule with carbons labeled numerically.......... 4

Figure 1.2: Double strand DNA sequences................................................................ 4

Figure 1.3: Central Dogma Of Molecular Biology ................................................... 8

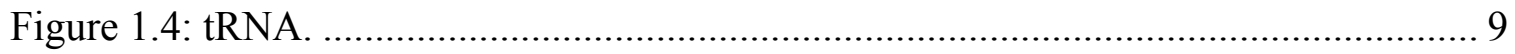

Figure 1.5: Sequence ladder by radioactive sequencing compared to fluorescent peaks. 12

Figure 1.6: An overview of how ESTs are constructed.......................................... 16

Figure 1.7: Two types of pseudogenes: processed pseudogenes and unprocessed

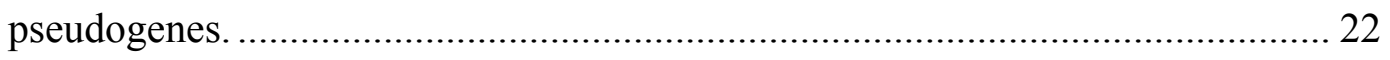

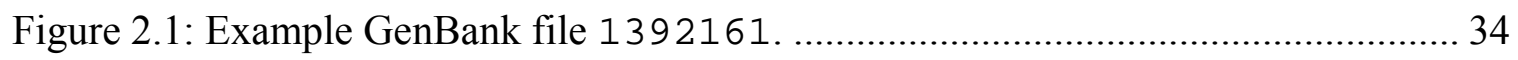

Figure 2.2: Example FASTA format 1392161................................................. 35

Figure 3.1: Cumulative results of wuBLAST and ncbiBLAST in EST vs. EST

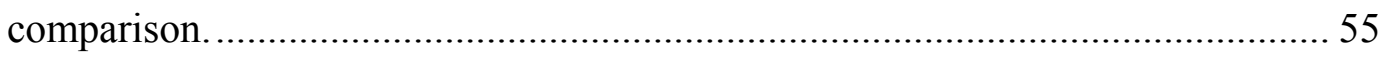

Figure 3.2: est166 target database vs. est136 and est147 query sequences. ................... 57

Figure 3.3: est166 target database vs. est241 and est176 query sequences....................5 57

Figure 3.4: Cumulative results of wuBLAST and ncbiBLAST using chromosome vs.

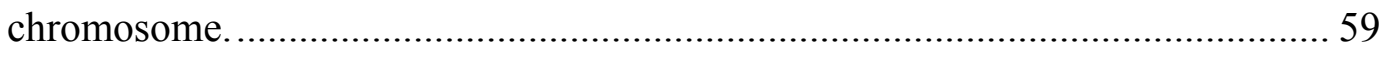

Figure 3.5: chr22 target database vs. est136 and est147 query sequences. .................... 60

Figure 3.6: chr1 target database vs. est136 and est147 query sequences. ....................... 60

Figure 3.7: Cumulative results of wuBLAST and ncbiBLAST for EST vs. chromosome. 
Figure 3.8: est166 target database vs. est136 query sequence in mpiBLAST. 64

Figure 3.9: chr1 target database vs. est136 query sequence in mpiBLAST. 64

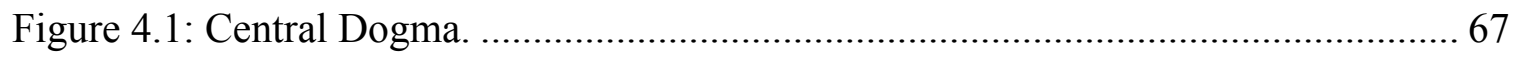

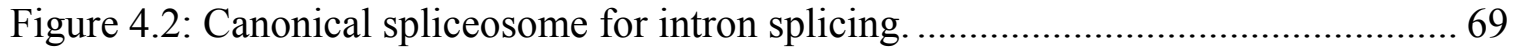

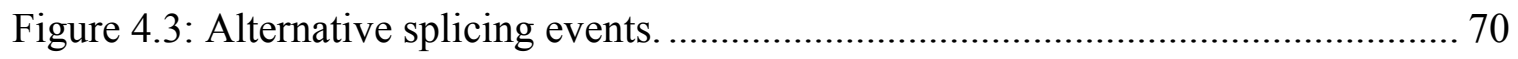

Figure 4.4: General scheme of cyclic 3', 5' nucleotide metabolism.............................. 77

Figure 4.5: PDE4 isoform diversity: long, short, super-short and dead-short................ 79

Figure 4.6: Process flow chart in computational detecting section. ............................. 83

Figure 4.7: Tissue distribution of ESTs for each of the four PDE4 families.................. 90

Figure 4.8: PDE4A isoforms, EST patterns and sequenced regions............................. 94

Figure 4.9: PDE4B isoforms, multiple EST patterns and sequence hits. ....................... 98

Figure 4.10: PDE4C isoforms, multiple EST patterns and sequence hits. .................... 100

Figure 4.11: PDE4D isoforms, multiple EST patterns and sequence hits (chromosome 2).

Figure 4.12: PDE4D isoforms, multiple EST patterns and sequence hits (chromosome 2

RANDOM). 104

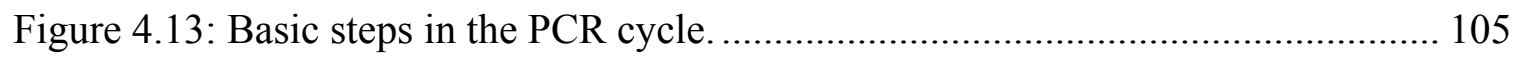

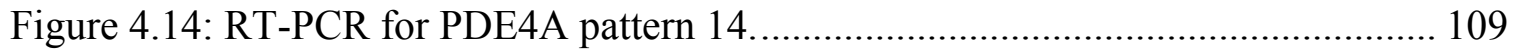

Figure 4.15: RT-PCR for PDE4B patterns 15 and 23.......................................... 109

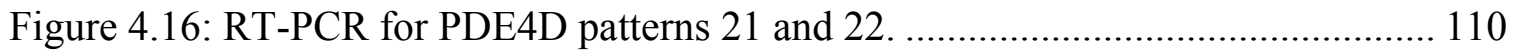

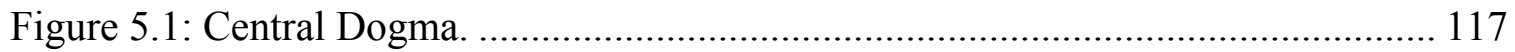

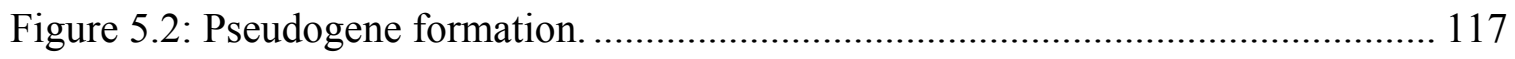

Figure 5.3: Flow chart of Sakai et al. method for processed pseudogene detection....... 122 
Figure 5.4: Mutations occurring in two homologous sequences.

Figure 5.5: Comparison between ancestral sequence and descendant sequence (1)..... 132

Figure 5.6: Comparison between ancestral sequence and descendant sequence (2)..... 133

Figure 5.7: Similar synonymous mutation vs. nonsynonymous mutation..................... 134

Figure 5.8: Process for pseudogene detection................................................... 136

Figure 5.9: Sample blastn result for GI 5555714........................................... 138

Figure 5.10: Flow chart for filtering blastn results. .......................................... 140

Figure 5.11: Sample blastn result GI no. 18288550...................................... 142

Figure 5.12: Sample EXALIN result for GI 18288550......................................... 142

Figure 5.13: Sample EXALIN result for GI 18288550..................................... 143

Figure 5.14: Flow chart for removing EXALIN redundant records........................... 144

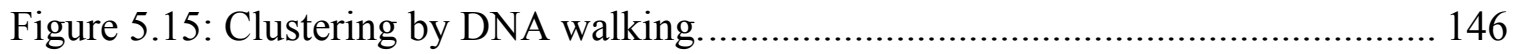




\section{CHAPTER I}

\section{INTRODUCTION}

Since the laws of inheritance were described by the Augustinian monk Gregor Mendel in 1866 (1-4), the genomic basis for heredity has been a topic of interest. The discovery of nucleic acids by Friedrich Miescher (5) in 1869 moved the study of genetics to a molecular level. Fueled by the basic 'truth' of the Central Dogma of Molecular Biology (6), molecular biologists have worked towards understanding the complex relationship between deoxyribonucleic acids (DNA), ribonucleic acids (RNA) and amino acids as they create proteins providing structure, function and regulation to a biological system.

The possibility of decoding the genomic DNA for a species through DNA sequencing has led to the ability to glimpse at the genetic blueprint for an individual. The vast amount of genomic data resulting from projects such as the human genome project provides a treasure chest of data waiting to be explored. This has led to the emergence of the field of Bioinformatics to provide computational means for handling and mining such data. The focus of this dissertation is to provide Bioinformatics' approach to understanding the complexity involved in gene regulation. In order to adequately address the problem at hand, the rest of this chapter is devoted to a background on basic genetics, molecular biology, DNA sequencing, genes, and bioinformatics, as well as a brief introduction into three interwoven projects dealing with gene regulation. 


\subsection{Basic Genetics}

The field of genetics traces its roots to 1866 with the published observations of the Augustinian monk Gregor Mendel (1-4). Mendel performed pea plant experiments to study the inheritance of genetic traits, such as tall vs. dwarf plants, wrinkled vs. round peas, violet vs. white flowers, etc. He concluded that the characteristics of living organisms are passed from generation to generation through replication.

Several major discoveries since have advanced the study of genetics at a molecular level. In 1869, nucleic acids were isolated and studied by Friedrich Miescher (5). In 1944, Avery, MacLeod and McCarty determined that DNA is the carrier of hereditary information $(7 ; 8)$ through four nucleic acid bases: adenine $(A)$, cytosine $(\mathrm{C})$, guanine $(\mathrm{G})$ and thymine $(\mathrm{T})$. This tetranucleotide hypothesis was verified in the late 1940s and early 1950s by Erwin Chargaff and his colleagues $(9 ; 10)$. In 1953, Watson and Crick discovered the structure of a double helix for DNA (11). These discoveries opened the door to many more advances in genetics over the past 60 years in the field of molecular biology, the branch of life science which studies cells at a molecular level, including nucleic acids, amino acids, proteins and fundamental biochemical reactions within a cell.

\subsection{DNA}

Deoxyribonucleic acid, or DNA, is composed of four nucleic acid bases adenine $(A)$, cytosine $(C)$, guanine $(\mathrm{G})$ and thymine $(\mathrm{T})$ - which are arranged in different ways to form sequences. The International Union of Pure and Applied Chemistry (IUPAC) and the International Union of Biochemistry (IUB) published a code in 1985 for 
representing DNA sequences (12) (Table 1.1). A single letter signifies a single nucleotide or nucleotide alternative, as shown in columns one and two.

Table 1.1: IUPAC-IUB Nucleic Acid and Amino Acid Codes

\begin{tabular}{|c|c|c|c|c|}
\hline \multicolumn{2}{|r|}{ Nucleotide Acid Codes } & \multicolumn{3}{|c|}{ Amino Acid Codes } \\
\hline Code & Base & Full name & $\begin{array}{c}\text { 3-letter } \\
\text { abbreviation }\end{array}$ & $\begin{array}{c}\text { 1-letter } \\
\text { abbreviation }\end{array}$ \\
\hline A & Adenine & Glycine & GLY & $\mathrm{G}$ \\
\hline $\mathrm{C}$ & Cytosine & Alanine & ALA & A \\
\hline G & Guanine & Valine & VAL & V \\
\hline $\mathrm{T}$ & $\begin{array}{l}\text { Thymine in DNA; Uracil in } \\
\text { RNA }\end{array}$ & Leucine & LEU & $\mathrm{L}$ \\
\hline M & $\mathrm{A}$ or $\mathrm{C}$ & Isoleucine & ILE & I \\
\hline $\mathrm{R}$ & $A$ or $G$ & Phenylalanine & PHE & $\mathrm{F}$ \\
\hline $\mathrm{W}$ & $\mathrm{A}$ or $\mathrm{T}$ & Proline & PRO & $\mathrm{P}$ \\
\hline $\mathrm{S}$ & $\mathrm{C}$ or $\mathrm{G}$ & Serine & SER & $\mathrm{S}$ \\
\hline $\mathrm{Y}$ & $\mathrm{C}$ or $\mathrm{T}$ & Threonine & THR & $\mathrm{T}$ \\
\hline K & $\mathrm{G}$ or $\mathrm{T}$ & Cysteine & CYS & $\mathrm{C}$ \\
\hline V & A or $\mathrm{C}$ or $\mathrm{G}$; not $\mathrm{T}$ & Methionine & MET & M \\
\hline $\mathrm{H}$ & $\mathrm{A}$ or $\mathrm{C}$ or $\mathrm{T}$; not $\mathrm{G}$ & Tryptophan & TRP & $\mathrm{W}$ \\
\hline $\mathrm{D}$ & $\mathrm{A}$ or $\mathrm{G}$ or $\mathrm{T}$; not $\mathrm{C}$ & Tyrosine & TYR & $\mathrm{Y}$ \\
\hline B & $\mathrm{C}$ or $\mathrm{G}$ or $\mathrm{T}$; not $\mathrm{A}$ & Asparagine & ASN & $\mathrm{N}$ \\
\hline \multirow[t]{6}{*}{$\mathrm{N}$} & $\mathrm{A}$ or $\mathrm{C}$ or $\mathrm{G}$ or $\mathrm{T}$ & Glutamine & GLN & Q \\
\hline & & Aspartic acid & ASP & $\mathrm{D}$ \\
\hline & & Glutamic acid & GLU & $\mathrm{E}$ \\
\hline & & Lysine & LYS & K \\
\hline & & Arginine & ARG & $\mathrm{R}$ \\
\hline & & Histidine & HIS & $\mathrm{H}$ \\
\hline
\end{tabular}

As Watson and Crick revealed (11), DNA is structured as a double helix, where two strands are held together and rotated around a horizontal axis. These two strands are "anti-parallel," with one strand running from a 5' to 3' direction and the other strand 
running from 3' to 5', where 3' and 5' represent structural properties in nucleic acids ${ }^{1}$. The two strands are held together by the energy of hydrogen bonds in complementary sequences where an A always pairs with a $T$, and a $C$ always pairs with a $G$ (and viceversa, see Figure 1.2).<smiles>O[14CH2][14CH](O)[14CH2]O</smiles>

Figure 1.1: A diagram of a sugar-ring molecule with carbons labeled numerically.

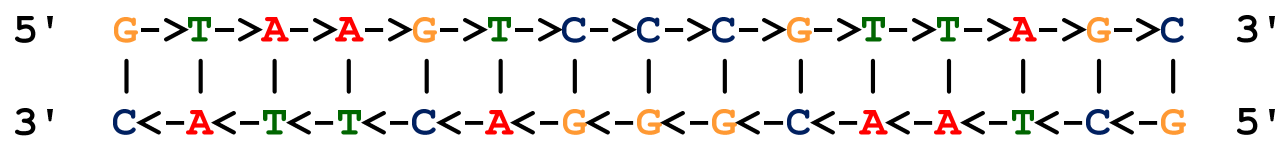

Figure 1.2: Double strand DNA sequences.

\subsection{RNA}

RNA stands for ribonucleic acid. Three of the four DNA bases are the same in RNA, with the exception that thymine (T) is substituted by uracil (U). Unlike DNA, RNA can leave the nucleus of a cell to perform functions and is normally a single-stranded molecule. There are three major, functionally-distinct varieties of RNA: messenger RNA (mRNA), ribosomal RNA (rRNA) and transfer RNA (tRNA). mRNA is involved in the

\footnotetext{
${ }^{1}$ Directionality in molecular biology refers to the end-to-end chemical orientation of a single strand of nucleic acid. The chemical convention of naming carbon atoms in the nucleotide sugar-ring numerically gives rise to a 5' end and a 3' end (usually pronounce "five prime end" and "three prime end"). Therefore, the 5' end designates the end of the DNA or RNA strand that has the fifth carbon in the sugar-ring of the (deoxy)ribose at its terminus. The 3' end has the third carbon in the sugar-ring as its terminus (Figure 1.1).
} 
transmission of DNA information. rRNA functions in the synthesis process of producing proteins. tRNA enables another part of protein synthesis by bringing together chains of amino acids (13). There are some other types of RNAs, such as RNA interference (RNAi), microRNA (miRNA) and silencing RNA (siRNA). They will be discussed in Section 1.6, Central Dogma.

\subsection{Amino Acids}

Amino acids are the basic elements of proteins. They form short polymer chains called peptides or longer sequences called polypeptides. In an mRNA sequence, every three nucleic acids form a $\operatorname{codon}^{2}$ that describes one amino acid. Since there are four nucleic acids, A, C, G and T, a codon can have up to $4 \times 4 \times 4$ or 64 different combinations, yet there are only twenty common amino acids. In 1962, Marshall Nirenberg (14-16) uncovered the genetic code, which allows for multiple codons to code for the same amino acid as shown in Table 1.2. During the translation process, a sequence begins with the initiator codon AUG and ends with any of the three terminator codons: UAA, UAG or UGA.

\footnotetext{
${ }^{2} \mathrm{~A}$ codon is a sequence of three adjacent nucleotides constituting the genetic code that determines the insertion of a specific amino acid in a polypeptide chain during protein synthesis or gives the signal to start or stop protein synthesis.
} 
Table 1.2: The Genetic Code (RNA to Amino Acids)

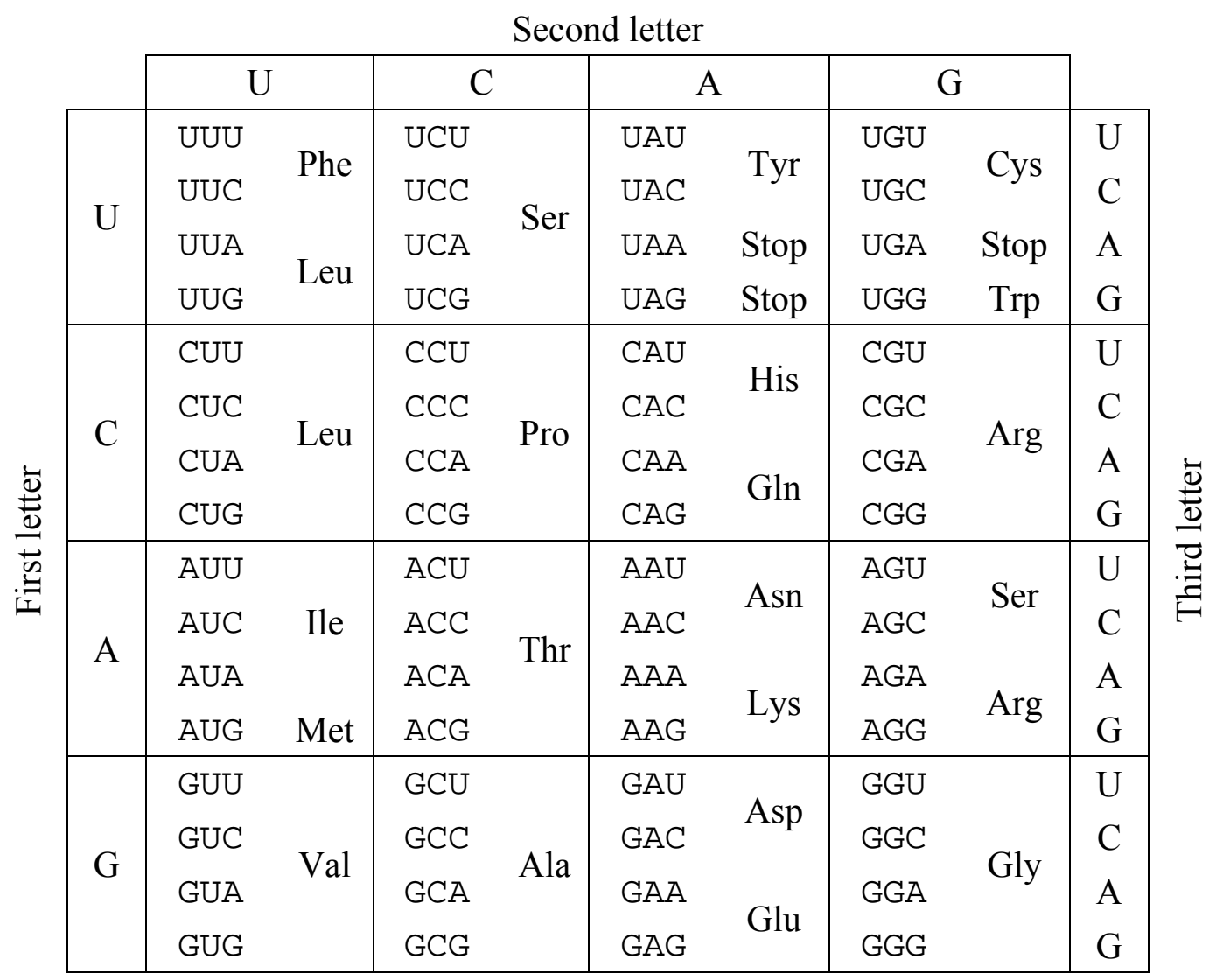


...CH-CO-NH... is the common chemical composition of all twenty amino acids, where $\mathrm{C}$ stands for carbon, $\mathrm{H}$ for hydrogen, $\mathrm{O}$ for oxygen and $\mathrm{N}$ for nitrogen. Attached to each $\mathrm{CH}$ is attached a small group of atoms - often called $\mathrm{R}$ by chemists, where $\mathrm{R}$ is a side-chain in the molecule. The side-chains provide for differentiation for the common twenty amino acids are listed in Table 1.3.

Table 1.3: Structure and Classification of the 20 Common Amino Acids

\begin{tabular}{|c|c|c|c|}
\hline One Letter & $\begin{array}{l}\text { Three } \\
\text { Letters }\end{array}$ & Name & Linear Structure \\
\hline$A$ & Ala & Alanine & $\mathrm{CH} 3-\mathrm{CH}(\mathrm{NH} 2)-\mathrm{COOH}$ \\
\hline $\mathrm{C}$ & Cys & Cysteine & $\mathrm{HS}-\mathrm{CH} 2-\mathrm{CH}(\mathrm{NH} 2)-\mathrm{COOH}$ \\
\hline $\mathrm{D}$ & Asp & Aspartic acid & $\mathrm{HOOC}-\mathrm{CH} 2-\mathrm{CH}(\mathrm{NH} 2)-\mathrm{COOH}$ \\
\hline $\mathrm{E}$ & Glu & Glutamic acid & $\mathrm{HOOC}-(\mathrm{CH} 2) 2-\mathrm{CH}(\mathrm{NH} 2)-\mathrm{COOH}$ \\
\hline $\mathrm{F}$ & Phe & Phenylalanine & $\mathrm{Ph}-\mathrm{CH} 2-\mathrm{CH}(\mathrm{NH} 2)-\mathrm{COOH}$ \\
\hline G & Gly & Glycine & $\mathrm{NH} 2-\mathrm{CH} 2-\mathrm{COOH}$ \\
\hline $\mathrm{H}$ & His & Histidine & $\mathrm{NH}-\mathrm{CH}=\mathrm{N}-\mathrm{CH}=\mathrm{C}-\mathrm{CH} 2-\mathrm{CH}(\mathrm{NH} 2)-\mathrm{COOH}$ \\
\hline 1 & Ile & Isoleucine & $\mathrm{CH} 3-\mathrm{CH} 2-\mathrm{CH}(\mathrm{CH} 3)-\mathrm{CH}(\mathrm{NH} 2)-\mathrm{COOH}$ \\
\hline $\mathrm{K}$ & Lys & Lysine & $\mathrm{H} 2 \mathrm{~N}-(\mathrm{CH} 2) 4-\mathrm{CH}(\mathrm{NH} 2)-\mathrm{COOH}$ \\
\hline L & Leu & Leucine & $(\mathrm{CH} 3) 2-\mathrm{CH}-\mathrm{CH} 2-\mathrm{CH}(\mathrm{NH} 2)-\mathrm{COOH}$ \\
\hline M & Met & Methionine & $\mathrm{CH} 3-\mathrm{S}-(\mathrm{CH} 2) 2-\mathrm{CH}(\mathrm{NH} 2)-\mathrm{COOH}$ \\
\hline $\mathrm{N}$ & Asn & Asparagine & $\mathrm{H} 2 \mathrm{~N}-\mathrm{CO}-\mathrm{CH} 2-\mathrm{CH}(\mathrm{NH} 2)-\mathrm{COOH}$ \\
\hline$P$ & Pro & Proline & $\mathrm{NH}-(\mathrm{CH} 2) 3-\mathrm{CH}-\mathrm{COOH}$ \\
\hline Q & Gln & Glutamine & $\mathrm{H} 2 \mathrm{~N}-\mathrm{CO}-(\mathrm{CH} 2) 2-\mathrm{CH}(\mathrm{NH} 2)-\mathrm{COOH}$ \\
\hline $\mathrm{R}$ & $\operatorname{Arg}$ & Arginine & $\begin{array}{l}\mathrm{HN}=\mathrm{C}(\mathrm{NH} 2)-\mathrm{NH}-(\mathrm{CH} 2) 3-\mathrm{CH}(\mathrm{NH} 2)- \\
\mathrm{COOH}\end{array}$ \\
\hline$S$ & Ser & Serine & $\mathrm{HO}-\mathrm{CH} 2-\mathrm{CH}(\mathrm{NH} 2)-\mathrm{COOH}$ \\
\hline $\mathrm{T}$ & Thr & Threonine & $\mathrm{CH} 3-\mathrm{CH}(\mathrm{OH})-\mathrm{CH}(\mathrm{NH} 2)-\mathrm{COOH}$ \\
\hline $\mathrm{V}$ & Val & Valine & $(\mathrm{CH} 3) 2-\mathrm{CH}-\mathrm{CH}(\mathrm{NH} 2)-\mathrm{COOH}$ \\
\hline W & Trp & Tryptophan & $\mathrm{Ph}-\mathrm{NH}-\mathrm{CH}=\mathrm{C}-\mathrm{CH} 2-\mathrm{CH}(\mathrm{NH} 2)-\mathrm{COOH}$ \\
\hline Y & Tyr & Tyrosine & $\mathrm{HO}-\mathrm{Ph}-\mathrm{CH} 2-\mathrm{CH}(\mathrm{NH} 2)-\mathrm{COOH}$ \\
\hline
\end{tabular}

Abbreviations: C, carbon; H, hydrogen; O, oxygen; N, nitrogen; S, sulfur;

$\mathrm{Ph}$, phenyl group or phenyl ring 


\subsection{Proteins}

Proteins are linear polymers built from amino acids. They carry out all of a cell's activities. Proteins serve different roles in a cell. As enzymes, proteins can accelerate the rate of metabolic reactions. As hormones, proteins perform regulatory functions inside a cell, such as activating genes. As membrane receptors and transporters, proteins determine what types of substances enter or leave the cell and give instructions on how a cell should react. There are many additional functions provided by proteins (17).

The specific amino acid sequence forming a protein gives the cell its unique property. Proteins may work alone in a cell or they can also work together to achieve a particular function. Proteins will often associate to form stable complexes in the cell.

\subsection{Central Dogma of Molecular Biology}

The journey from genes to proteins involves several steps in the Central Dogma of Molecular Biology. In transcription, a DNA gene is copied to a primary RNA transcript. During the splicing process, exons (coding regions) join together and introns (non-coding regions) are discarded, turning the primary RNA transcript into messenger RNA (mRNA). Later, through the process of translation, mRNA is transformed into a protein sequence. This Central Dogma was first described by Francis Crick in 1958 (6).

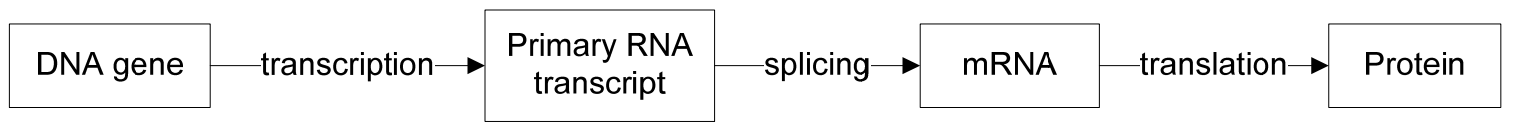

Figure 1.3: Central Dogma Of Molecular Biology. 
RNA is synthesized in the nucleus of cells. When a promoter ${ }^{3}$ is recognized, RNA polymerase ${ }^{4}$ binds to it, catalyzes the insertion to open the DNA double helix strands, and starts to make an RNA transcript. The process will not stop until the enzyme traverses the entire gene and meets a termination signal. Then the transcribed RNA molecule is released from the DNA.

The initial RNA transcript passes through an excision and rejoining process, called splicing. The introns are removed and the exons are recombined together, creating mRNA. This mRNA associates with the ribosome and presents a series of codons that will only match to specific amino acids. Each amino acid is attached to a tRNA molecule. The tRNA with three consecutive ribonucleotides that complement the codon will be paired with the section of mRNA (See Figure 1.4). The amino acids combine to form protein $(18 ; 19)$.

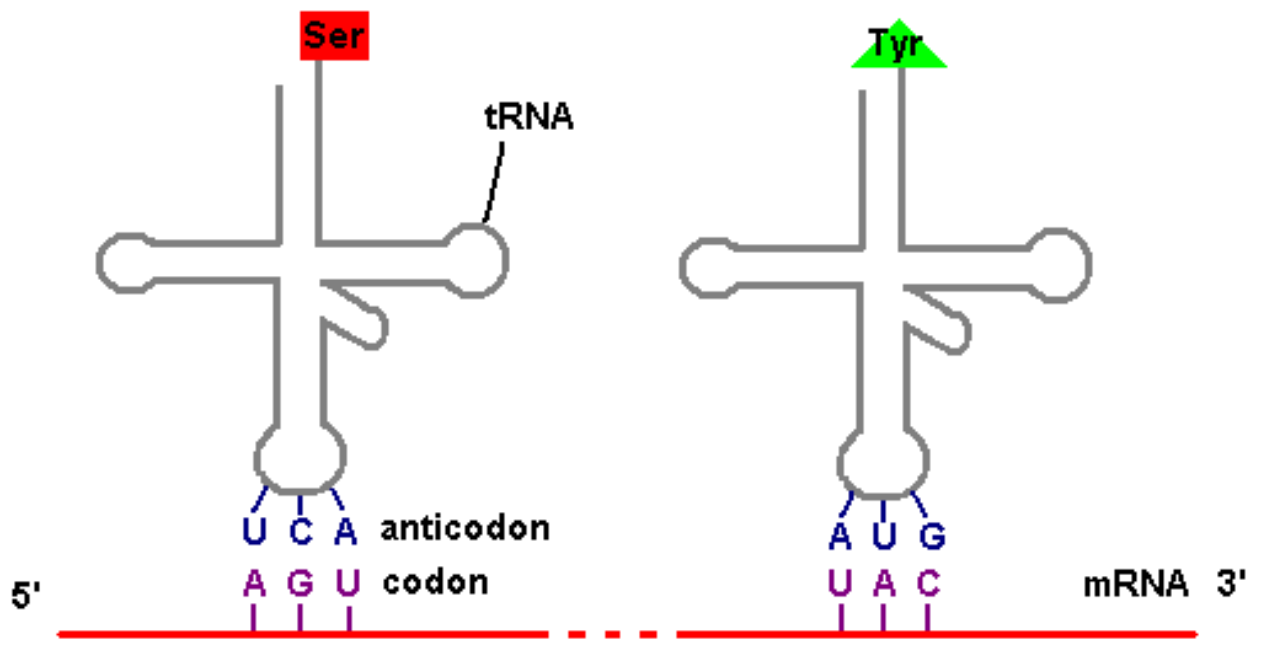

Figure 1.4: tRNA.

\footnotetext{
${ }^{3}$ A promoter is a sequence of DNA that specifies the sites of transcription initiation for an RNA polymerase.

${ }^{4}$ A polymerase is an enzyme that can synthesize new DNA strands using a DNA template while several such enzymes exist.
} 
The concept of the Central Dogma has been challenged by a series of discoveries revealing the complexity of gene expression. The first challenge came in 1970 with the discovery of reverse transcription. Reverse transcription allows a double stranded DNA molecule to be curated from a single strand RNA template. At first, reverse transcription sequences was found mostly in viruses. But after large-scale sequencing techniques were established, close to half of the sequences in the human genome were found to be retroelements and their remnants (20).

In 1988, the discovery of RNA interference (RNAi) by Andrew Fire and Craig Mello solved the problem of retroelement recognition (21-26). They found that it is common for animals and plants to utilize enzymatic machinery in order to silence translation. Short RNAs (22-25 base pairs in length), called small interfering RNA (siRNA), can traverse intracellular spaces to enter cells and trigger degeneration of homologous RNAs before they are translated to proteins. Both RNAi and siRNA are microRNA (miRNA).

In addition, prions, the microscopic protein particles similar to viruses but lacking nucleic acids, may be the simplest exception to the Central Dogma. Prions have been thought to be the infectious agent responsible for scrapie and certain other degenerative diseases of the nervous system, such as Creutzfeldt-Jakob and mad cow disease.

The mystery of non-coding RNA is still a hot topic. RNAi is the most recent RNA-based phenomenon to catch biologists' attention. There may be more secrets unveiled to challenge the traditional view of a Central Dogma in the near future (20). 


\subsection{DNA Sequencing}

DNA sequencing is the method used to determine the order of the nucleotide bases, A, C, G and T, in a DNA oligonucleotide ${ }^{5}$. The methods were first developed in 1977 with the publication of two approaches. One was developed by Frederick Sanger (27) which became known as Sanger sequencing. It is based on enzymatic synthesis from a single-strand DNA template with chain termination using four dideoxynucleotides $(\text { ddNTPs })^{6}$ as chain terminators - terminator $\mathrm{A}$, terminator $\mathrm{C}$, terminator $\mathrm{G}$, and terminator T. Another method was designed by Maxam and Gilbert (28) that involved chemical degradation of end-radio-labeled DNA fragments. Both methods depended on four-lane, high-resolution polyacrylamide gel electrophoresis ${ }^{7}$ to separate the labeled fragments so the sequence can be read in a staggered ladder-like platform. The Sanger method allows for the DNA to be combined in a single experiment due to the unique dyes identifying each nucleotide. Therefore, it became the major DNA sequencing method (32).

In Sanger sequencing, the ddNTP lacks a 3'OH group. Thus, DNA polymerases cannot extend the template copy chain beyond the incorporated ddNTP. In other words, ddATP terminates chains at every A (adenine) occurrence in the sequences. The other three ddNTPs, ddCTP, ddGTP, and ddTTP, work in the same way for their respective bases. They are placed in a test tube with a DNA fragment and DNA polymerase. The strands then placed on a gel to run electrophoresis. Since the ddNTP is phosphorescently

\footnotetext{
${ }^{5}$ An oligonucleotide (or oligo) is a short polymer segment of RNA or DNA, length ranges from two to twenty bases.

${ }^{6}$ ddNTP, which stands for dideoxynucleotide, represents one of the four portions: ddATP, ddCTP, ddGTP and ddTTP.

${ }^{7}$ Electrophoresis is a method of separating substances, especially proteins, and analyzing molecular structure based on the rate of movement of each component in a colloidal suspension while under the influence of an electric field. This method was introduced by Arne Tiselius in 1937 for separating proteins in solution (29-31).
} 
labeled, the locations where the chain terminators were incorporated for one of the four particular nucleotide, is known and the DNA sequence can be determined based on the order of incorporated ddNTPs (32).

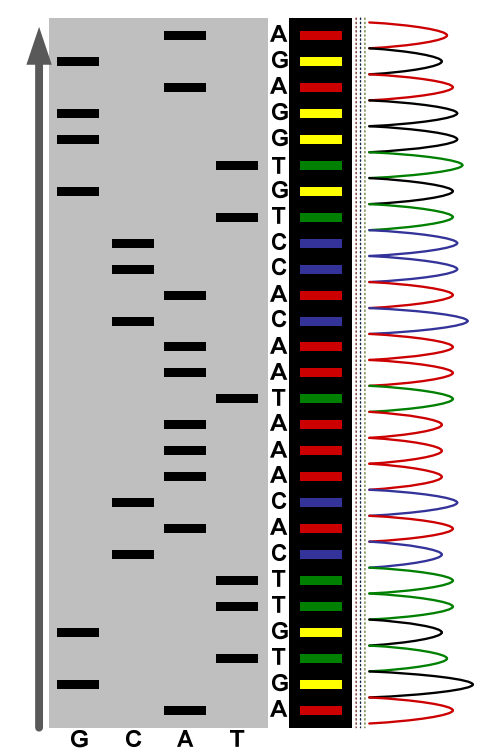

Figure 1.5: Sequence ladder by radioactive sequencing compared to fluorescent peaks.

\subsubsection{Evolution of Sequencing}

The demand for DNA sequence information has never been greater, especially with the popularity of the Human Genome Project (Section 1.7.3). However, the Sanger sequencing method is costly, time consuming and labor intensive. Therefore, several emerging technologies have tried to improve Sanger's method to deliver fast and affordable genome sequencing (33). These methods include traditional electrophoretic approaches (slab gel electrophoresis, capillary electrophoresis, and microfabricated capillary arrays) and nonelectrophoretic methods (pyrosequencing, massively parallel signature sequencing, and sequencing by MLDI-TOF mass spectrometry) (34). 
Acrylamide slab gel electrophoresis uses radioactive $\left({ }^{32} \mathrm{P}\right)$ labels and autoradiograms for the de novo sequencing of DNA fragments. The fluorescently labeled DNA fragments are loaded into acrylamide gel. An electric current to causes the fragments migrating through the gel to separate by the weight of the nucleic acids. Then a detection system scans these radioactive labeled bands and reconstructs the original nucleotide sequences. This method can process up to 96 samples in a 9- to 11-hour run and can read sequences ranging in length from 650 to 750 bases (34).

The capillary array electrophoresis (CAE) method applies the same process as slab gels, except CAE adds a single sample in each capillary to avoid tracking problems. In addition, CAE allows more rapid heat dissipation. Therefore, the CAE method accommodates higher operating voltages and produces a faster run time. The CAE method can run 128 capillaries at one time and provide readings 500 bases long (34).

Microfabricated capillary arrays tried to perform the sample preparation, thermal cycling and electrophoretic separation on a single chip. This method etches a very large number of capillaries onto a single wafer at a low cost for parallel operation. In addition, this method allows the instrument designer to control the size of the injected DNA plug. It can handle 16-channel devices at the same time and produce average read lengths over 450 bases (34).

In the 1980s and 1990s researchers started to work on finding nonelectrophoretic separation methods for sequencing. Pyrosequencing monitors the concentration of pyrophosphate (PPi) during real-time DNA synthesis. First, it anneals an oligonucleotide primer to a single-stranded DNA template in the presence of DNA polymerase. Then, the first of four deoxynucleotide triphosphates (for example, dATP) is added to the reaction 
mixture. When dATP encounters the right complement in the target sequence (adenine), the DNA polymerase releases PPi because the nucleotide reacts to the $3^{\prime}$ end of the nascent strand. The concentration of PPi in the system is monitored using enzymatic reactions. Next, dATP is removed and another deoxynucleotide triphosphate is added to repeat the process. Each of the other three deoxynucleotide triphosphates (dCTP, dGTP and dTTP) will release PPi in the presence of their respective nucleic acids (34).

Massively parallel signature sequencing (MPSS) is another sequencing strategy. This method examines cDNA libraries by combining the highly parallel nature of expression microarrays with the robust statistical digital output from gel-based sequencing. The process uses 32-mer tags to compose of strings of eight 4-mer "words". Each bead carries approximately 100,000 cDNA copies of a unique mRNA. These microbeads are sorted by a fluorescence-activated cell sorter. Then they are delivered to a micromachined flow cell to form a planar. Close to one million cDNA-coated beads can be analyzed in a single flow cell using conventional fluorescence microscopy (34).

Another method for sequencing DNA is matrix-assisted laser desorption ionization time-of-flight mass spectrometry (MALDI-TOF-MS). It extends concepts from mass spectrometry used to detect macromolecules. The DNA sample is treated with a short pulse of ultra-violet (UV) laser that is absorbed by the 3-hydroxypicolic acid to form ionized DNA fragments. These ions are extracted by a high-voltage pulse and accelerated in an electric field. A mass spectrum is generated and peaks identifying the weights for the ionized DNA fragments are evaluated by comparing them to the pre-built internal standards (34). 
One idea for the next-generation of sequencing is to create a factory-like sequencing center. This would involve automated capillary electrophoresis and analyzing the sequences in parallel (35).

Next-generation sequencing may also improve the length of reads. Generally speaking, sequencing-by-synthesis technology provides reads 400 bases long. Advances in combining the emulsion polymerase chain reaction (PCR) with pyrosequencing for the bias-free amplification of single DNA molecules will eliminate the competition of multiple templates with limited DNA polymerases. This process could be performed in parallel by generating a visual signal for processing by a computer system (35).

These improvements could reduce cost and allow assessment at functional levels. As a result, they would facilitate individual genome sequencing for personalized treatment (34-36).

\subsubsection{Expressed Sequence Tags}

The mRNA molecule is not stable outside of the cell. In order to preserve genetic information, mRNA can be reverse transcribed into complementary DNA (cDNA). The cDNA usually contains an oligo(dT) primer $^{8}$. This end of the cDNA derives from the polyadenyl (poly-A) tail ${ }^{9}$ at the end of the mRNA. The resulting cDNA is cloned into a vector. Expressed sequence tags (ESTs) are segments of cDNA that have been sequenced. Sequence of cDNA can be identified from either the beginning portion (which is called a 5' EST) or the end portion (which is called a 3' EST) $(18 ; 19 ; 37-39)$.

\footnotetext{
${ }^{8}$ A primer is a segment of DNA or RNA that is complementary to a given DNA sequence and that is needed to initiate replication by DNA polymerase.

${ }^{9}$ A polyadenyl (poly-A) tail is a sequence of 50 to 250 adenine (A) residues that are added to the 3' end of an mRNA sequence after being cleaved. This reaction is catalyzed by polyadenylate polymerase.
} 
An EST, which is usually 300 to 600 nucleotides long, represents a segment of a cDNA from a corresponding mRNA. It helps to identify unknown genes expressed in a given cell and can be used to map their positions within a genome.

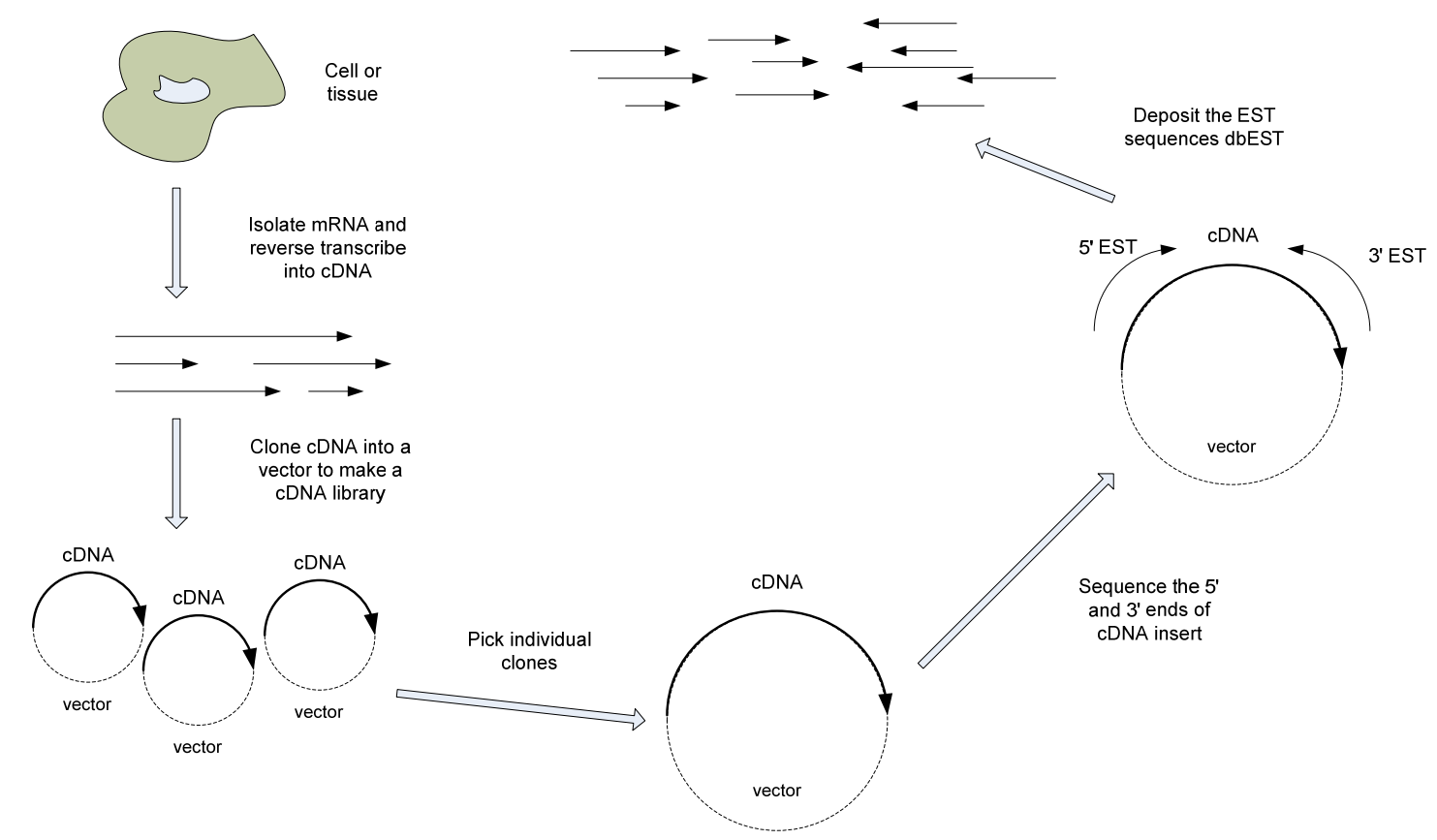

Figure 1.6: An overview of how ESTs are constructed.

\subsubsection{Human Genome Project (HGP)}

The push to map the human genome was initiated by a 1988 report from the National Academy of Science (NAS) Committee which maintained that the human genome could be sequenced in 15 years, plus an additional two years to fill in gaps and correct mistakes. The project was submitted to Congress where it was approved and received $\$ 3$ billion in funding. It began in 1990 (40).

The goals of the HGP were to "identify all the approximately 20,000-25,000 genes in human DNA, determine the sequences of the 3 billion chemical base pairs that make 
up human DNA, store this information in databases, improve tools for data analysis, transfer related technologies to the private sector, and address the ethical, legal, and social issues (ELSI) that may arise from the project" $(40 ; 41)$.

In February 2001, the work of the HGP and Celera published the first draft of the human genome (41-44). In 2003, 95\% of the gene-containing part of the human sequence was mapped to $99.99 \%$ accuracy.

After the human genome was available, researchers started to think about what could be done with this information. One option is to design personalized medicine for more effective treatment. In order to design an individualized medicine, however, researchers must be able to sequence an individual genome. On June $1^{\text {st }}, 2007,454$ Life Science Corporation in collaboration with scientists at the Human Genome Sequencing Center at Baylor College of Medicine (45), announced that they had sequenced James Watson's personal genome. Later at the end of the same month, the Cold Spring Harbor Laboratory (CSHL) announced that an online sequence browser for Watson's genome was available (46). Watson, who was the CSHL Chancellor and is currently serves as an advisor for the newly-formed Allen Institute for Brain Science ${ }^{10}$, said, "I am putting my genome sequence on line to encourage the development of an era of personalized medicine, in which information contained in our genomes can be used to identify and prevent disease and to create individualized medical therapies" $(45 ; 46)$.

\footnotetext{
${ }^{10}$ Allen Institute for Brain Science, located in Seattle, Washington, was founded in 2003 by philanthropists Paul G. Allen and Jody Allen Patton as a nonprofit corporation (501(c) (3)) and medical research organization. (http://www.alleninstitute.org/content/about_the_institute.htm)
} 


\subsubsection{ENCODE Project}

The ENCyclopedia of DNA Elements (ENCODE) project was initiated in September 2003. It brought several dozen laboratories together to identify the functional elements in the human genome. Two components - a pilot phase and a technology development phase - were proposed when the project started.

The aim of the pilot phase was to evaluate a variety of different methods for use in later stages. A portion of the genome equal to about $1 \%(30 \mathrm{MB})$ was carefully chosen and analyzed using new and existing methods. Transcripts and gene models, proteinbinding sites, epigenetic marks, promoters and enhancers, and DNA replication regions were the fundamental items involved in the research. The conclusions of this pilot project were published in June 2007 (47-56).

The technology development phase promoted several new technologies and protocols to generate high throughput data on functional elements for use in the ENCODE project. A third phase the production phase began in September 2007. A Data Coordination Center at the University of California at Santa Cruz (UCSC) was created to track, store and display the ENCODE data. In addition, a Data Analysis Center was established. All data generated by the ENCODE participants is released by the Data Coordination Center (49).

\subsection{Genes}

\subsubsection{What is a Gene?}

Genes are pieces of DNA that provide the fundamental physical and functional information of heredity. Through the processes of transcription and translation, the DNA 
of a gene controls the manufacturing of specific proteins for regular usage throughout all cells and organ systems. Each sequence, appearing in a particular location of a particular chromosome, encodes a unique function.

Genes include coding regions (exons) and non-coding regions (introns). Coding regions contain information used to specify amino acids in the synthesis of proteins. Noncoding regions do not. Genes are spliced to have alternative combinations of exons to produce various proteins in specific organs $(57 ; 58)$. This allows cells in different organs to serve different functions.

In 2007, Gerstein et al.(51) published a review of the changing definition of genes from the 1860s to the early 2000s, just before the ENCODE project (Section 1.3.4). Between the 1860s-1900s, each gene was treated as a discrete unit of heredity, based on the concept developed by Gregor Mendel in 1866 (1-4). The word 'gene' was introduced by Wilhelm Johannsen in 1909, by combining two Greek words, genesis (meaning 'birth') and geno (meaning 'origin'). Mendel's work was rediscovered by Correns, von Tschermak-Seysenegg and de Vries in 1900 (59-61).

In the $1910 \mathrm{~s}$, the gene became defined as a distinct locus ${ }^{11}$. Thomas Hunt Morgan demonstrated that genes are carried on chromosomes and are the mechanical basis of heredity. Hunt and his students (62) represented genes using a linear model from studying mutation in fruit flies (Drosophila melanogaster). The ability of genes to cross-over to each other was proportional to the distance that separated them. Mutations and recombination were two methodologies that early geneticists proposed, "so the gene was essentially a locus whose size was determined by' mutations that inactivated a trait of interest and by the size of the recombining regions."

\footnotetext{
${ }^{11}$ A locus is a position that a given gene occupies on a chromosome.
} 
In the 1940s, the gene was seen as a blueprint for a protein. This gave rise to the statement "one gene, one enzyme" and soon evolved to "one gene, one polypeptide." Hence, in the biochemical pathway, genes provided the information behind the individual molecules.

In the 1950s, after Muller published an observation of mutations caused by Xrays, the gene was described as a physical molecule. Later, in the mid 1950s, Hershey and Chase found from their study of bacteria that inheritance came from DNA and not protein. After Watson and Crick announced the three-dimensional structure of DNA, the gene was explained as transcribed code in the 1960s. Crick further presented a hypothesis of gene expression from nucleic acid to protein, which soon became known as the "Central Dogma" (Section 1.6). Molecular biology developed rapidly during this period of time.

Between the 1970s-1980s, Sanger developed sequencing and cloning technology (63). In parallel, computational tools were developed to utilize algorithms in order to identify genes based on sequential characteristics. Therefore, the gene became defined as an open reading frame $(\mathrm{ORF})$ sequence pattern.

After the HGP was announced, a gene became further defined as "a DNA segment that contributes to phenotype/function. In the absence of demonstrated function a gene may be characterized by sequence, transcription or homology" according to the Human Genome Nomenclature Organization (64).

After the ENCODE pilot project (Section 1.7.4), the definition suggested by the ENCODE consortium was concisely summarized as "the gene is a union of genomic sequences encoding a coherent set of potentially overlapping functional products" (51). 


\subsubsection{Alternative Splicing}

Alternative splicing is process of mRNA transcription through which the coding regions for a eukaryotic gene, called exons, are extracted and connected to produce alternative mRNA molecules. The event leads to a number of distinguishable proteins for a single transcriptional region. The resulting mRNA molecules, known as isoforms or splice variants, are becoming increasingly valued as an important biological method of producing complexity and diversity.

There are different kinds of alternative splicing: alternative promoter selection, alternative polyadenylation sites, exon skipping, exon retention, exon extension, exon truncation and intron retention. Alternative splicing is discussed in greater detail in Chapter 4.

\subsubsection{Pseudogenes}

A pseudogene is an inactive sequence of genomic DNA that shares sequence homology to a functional gene. In 1985, Vanin suggested "that pseudogenes (should) be used only to describe sequences found to be both related and defective" (65). Because of the high percentage of similarity to an existing decoded gene, pseudogenes are considered as evolutionary relatives to normally functioning genes.

Pseudogenes are created in two fundamentally different ways: retrotransposition and duplication of genomic DNA. Pseudogenes resulting from retrotransposition are called processed pseudogenes and pseudogenes resulting from DNA duplication are known as duplicated pseudogenes. Figure 1.7 provides a basic illustration of how 
processed and unprocessed pseudogenes are produced. Pseudogene detection and characterization are research interests of this dissertation discussed in Chapter 5.

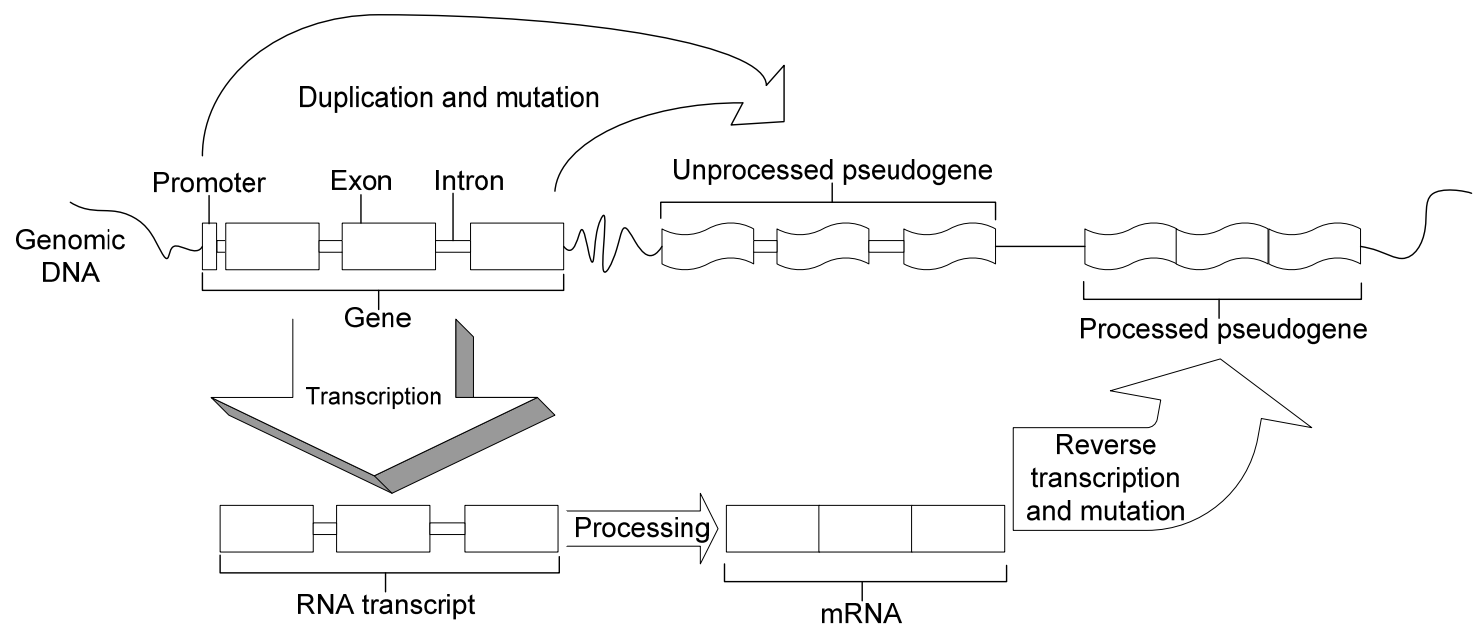

Figure 1.7: Two types of pseudogenes: processed pseudogenes and unprocessed pseudogenes.

\subsection{Bioinformatics and Computational Biology}

Bioinformatics is a compound word combining "bio" and "informatics." As the word suggests, this field of research is related to both biology and information. A related term, "Computational Biology," invokes the idea of using computers to perform calculations for biology.

From the perspective of computer science, biological data can be formalized into an alphabetic containing letters, such as A, C, G and T for DNA; A, C, G and U for RNA; or 20 letters for amino acids. This alphabet can then be used to produce "words" for sequences of DNA, RNA, or amino acids (66).

Bioinformatics and Computational Biology refer to applications of computers and computational techniques to deal with the storage and analysis of biological data (57). They use tools to implement algorithms into software to retrieve and analyze the 
biological data and manage data storage. Compared to other sciences, Bioinformatics and Computational Biology are relatively young disciplines.

In earlier years, "Bioinformatics" was used more frequently in Europe and the United Kingdom. Research in Bioinformatics centered on data management. Computational Biology, on the other hand, which was used more often in the United States, referred mostly to algorithm development. In recent years, the boundary between Bioinformatics and Computational Biology has become less clearly defined. Both fields now work on managing data and constructing algorithms to analyze it. Therefore, the research directions of these two fields are merging.

Bioinformatics and Computational Biology involve the disciplines of Chemistry, Biochemistry, Molecular Biology, Genetics, Genomics, Molecular Evolution, Computer Science, Statistics and Mathematics. Input from these disciplines allows the results of Bioinformatics to be interpreted in many different ways. Research in Computational Biology often includes system biology.

Major research efforts in Bioinformatics and Computational Biology involve sequence alignment, gene annotation, genome assembly, protein structure alignment, protein structure prediction, prediction of gene expression, protein-protein interactions, the modeling of evolution and analysis of the mutations in cancer. In summary, research efforts have progressed from sequence alignment to finding sequence similarity, verifying gene annotation, observing protein structure, and then even further to analyzing mutations and modeling the evolution of genes.

Luscombe, Greenbaum, and Gerstein (67) proposed that "the aims of bioinformatics are three-fold." The first aim is to provide the simplest way for researchers 
to access and submit their produced work. The second aim is to "develop tools and resources that aid in the analysis of data." The last aim is to use these established tools to analyze the data and give "results in a biologically meaningful manner."

\subsection{Introduction to Main Projects}

This dissertation utilizes techniques from Computational Biology and Bioinformatics for gene recognition. The following sections briefly describe the main projects for this research.

\subsubsection{Comparison of BLAST Software}

The computational power required to search exponentially growing databases, such as GenBank (68), has increased dramatically. Several tools were developed in the past few years for rapid DNA and protein sequence comparison. One of the most widely used sequence alignment tools, known as BLAST (Basic Local Alignment Search Tool), was released in 1990 by the researchers at the National Center for Biotechnology Information (NCBI) (69). In 1995, one of the original authors of BLAST began development of the algorithm at Washington University (WU), independent of the NCBI effort. In the same year, the first release of WU BLAST (version 1.4) was released. Since the original development, a number of changes have been incorporated (70). In order to evaluate the effectiveness of BLAST for sequence comparison at a nucleotide level, a study was performed of three current versions of BLAST software: NCBI BLAST, WU BLAST and mpiBLAST. 
In general, WU BLAST was found to be most efficient when the database and query sequences are unknown. NCBI BLAST appeared to work best when the database contains a small number of sequences, while mpiBLAST showed the power of database distribution when the number of bases per target database is large. The optimal number of compute nodes in mpiBLAST varies depending upon the database, yet in the cases studied, remained surprisingly low. A detailed description of the project will be discussed in Chapter 3, Sections 3.4 to 3.8.

\subsubsection{Alternative Splicing Recognition}

Studies in alternative splicing have found that a gene may produce more than one protein in order to fulfill different requirements and needs in cells. One gene with a large number of known splice variants is phosphodiesterase 4 (PDE4).

PDE4 is an enzyme that has been targeted for inflammatory disorders, including asthma, chronic obstructive pulmonary disease (COPD), and central nervous system (CNS) injury/disease. PDE4 has a large number of potential isoforms with apparent tissue and functional specificity. Potential roles of the different PDE4 isoforms within the CNS and other tissue types were studied using all four known rat PDE4 paralogs (PDE4A, PDE4B, PDE4C and PDE4D).

In order to see how ESTs can be used to detect novel isoforms and tissue specificity with alternative splicing for PDE4, a computational method to align rat ESTs to the corresponding chromosomal and mRNA sequences was developed. The splice pattern and tissue type for each EST was examined in order to gain more insight into 
PDE4 isoform tissue specificity. Further discussion will be provided in Chapter 4, Sections 4.4 to 4.9 .

\subsubsection{Pseudogene Detection and Characterization}

As genes provide the heredity information for an organism, pseudogenes are regions sharing sequence homology to the functional genes yet are unable to operate as genes. Pseudogenes can create difficulties in detecting genes in silico. A method to uncover pseudogenes and to differentiate between functional genes and pseudogenes is desired. Chapter 5 discusses an algorithm which was designed and implemented to detect and differentiate pseudogene.

\subsection{Organization of Dissertation}

The focus of this dissertation is to design and implement algorithms to analyze data computationally and the use of ESTs through three different but inter-related projects - comparison of current BLAST software in nucleotide sequences, alternative splice pattern recognition, and pseudogene detection and characterization. Chapter 2 introduces the necessary data components. Chapter 3 reviews the preprocessing methods for sequence alignment and examines the comparison of current BLAST software. Chapter 4 discusses the computational method for alternative splice pattern recognition and gives a case study in discovering rat PDE4 sequences. Chapter 5 details the methodology of pseudogene detection and characterization and provides a scoring scheme of distinguish genes and pseudogenes. Chapter 6 connects the three projects 
discussed in Chapters 3 to 5 to draw conclusions regarding computational analysis for understanding gene regulation and provide ideas for extending the research in the future. 


\section{CHAPTER II}

\section{DATA SOURCES AND DATA PREPROCESSING}

Since the invention of automated genome sequencers, a large amount of biological sequence data has been made available to the public. GenBank (68) was established in 1982 by the National Institutes of Health (NIH) to help advance scientific discovery worldwide. According to a news released from NIH, dated April 3, 2008, this comprehensive database has collected sequences from more than 240,000 organisms. The number of base pairs in GenBank doubles about every 19 months. Recently, the public collection of DNA and RNA sequences has reached approximately 110 million sequences and 200 billion base pairs (71). This data is maintained and exchanged on a daily basis under the International Nucleotide Sequence Database Collaboration (INSDC), which includes GenBank at the National Center for Biotechnology Information (NCBI), European Molecular Biology Laboratory (EMBL) and the DNA Data Bank of Japan (DDBJ). Every two months, a new version of GenBank is released. The three main sources of data for the three projects discussed in dissertation are ESTs, mRNA and genomic sequences from the GenBank and University California Santa Cruz (UCSC).

\subsection{Sequence Data}

\subsection{1 dbEST}

dbEST is division of GenBank that collects ESTs submitted from around the world. It contains sequence data and its annotation information from numerous organisms 
(72). Newly discovered ESTs are submitted to dbEST daily. About every two weeks, dbEST releases an update on the number of entries. In addition, approximately every two months, GenBank releases a new version of flat format files which allow researchers to download the newest data to their workstations. Table 2.1 provides the top 25 organisms in GenBank/dbEST released on May 30, 2008

(http://www.ncbi.nlm.nih.gov/dbEST/dbEST_summary.html). The total number of public entries in this release was $52,858,766$. The human genome had the largest number of entries for all organisms (15.40\%).

Table 2.1: Top 25 Organisms in dbEST (as of May 30, 2008)

\begin{tabular}{lr}
\hline Organism & \# of entries \\
\hline Homo sapiens (human) & $8,137,747$ \\
Mus musculus + domesticus (mouse) & $4,850,258$ \\
Arabidopsis thaliana (thale cress) & $1,526,133$ \\
Bos taurus (cattle) & $1,517,053$ \\
Sus scrofa (pig) & $1,476,357$ \\
Zea mays (maize) & $1,462,614$ \\
Danio rerio (zebrafish) & $1,379,829$ \\
Xenopus tropicalis (western clawed frog) & $1,271,375$ \\
Oryza sativa (rice) & $1,220,876$ \\
Triticum aestivum (wheat) & $1,051,465$ \\
Ciona intestinalis (sea squirt) & 996,088 \\
Rattus norvegicus + sp. (rat) & 895,894 \\
Xenopus laevis (African clawed frog) & 677,784 \\
Oryzias latipes (Japanese medaka) & 609,699 \\
Gallus gallus (chicken) & 599,383 \\
Drosophila melanogaster (fruit fly) & 573,212 \\
Brassica napus (oilseed rape) & 567,177 \\
Hordeum vulgare + subsp. vulgare (barley) & 478,734 \\
Salmo salar (Atlantic salmon) & 433,337 \\
Glycine max (soybean) & 394,370 \\
Canis familiaris (dog) & 365,909 \\
Vitis vinifera (wine grape) & 352,984 \\
Branchiostoma floridae (Florida lancelet) & 346,107 \\
Pinus taeda (loblolly pine) & 334,502 \\
Physcomitrella patens subsp. Patens & 328,628 \\
\hline
\end{tabular}




\subsubsection{Reference Sequence (RefSeq)}

The Reference Sequence (RefSeq) database (73-76) is a collection of annotated DNA, RNA and protein sequences from major organisms, including plasmids, organelles, viruses, archaea, bacteria, and eukaryotes. RefSeq contains data extracted from GenBank, and involves varying levels of computational and experimental verification from authoritative groups. Each RefSeq provides non-redundant information on genomic location, sequence and text annotation as well as genome annotation ${ }^{12}$, gene characterization, mutation analysis, expression studies and polymorphic discovery. In addition, the database supports sequence standards for genomes and transcripts or proteins, providing a foundation for unambiguous comparison of functional information (76).

RefSeq is updated daily with new records added to the collection, sequence updates, and annotation changes. The May 2008 RefSeq collection (Release 29, dated 59-2008, http://www.ncbi.nlm.nih.gov/RefSeq/) was comprised of sequences from over 5,000 distinct taxonomies, ranging from viruses to bacteria to eukaryotes. It included whole chromosomes, organelles, plasmids, viruses, transcripts and more than 5,400,000 proteins. Table 2.2 shows the number of species in each sub-directory and the counts of accessions and length per type of molecule.

\footnotetext{
${ }^{12}$ Genome annotation is a process of assigning biological information to sequences. The process is based on two main steps: identifying elements on the genome and adding the biological information to the elements. An annotation is a note added by way of commentary and explanation. Once a genome is sequenced, it needs to be annotated and clarified.
} 
Table 2.2: Statistical Information of RefSeq Sequences (as of May 9, 2008)

\begin{tabular}{|c|c|c|c|c|}
\hline Species & \# of organisms & & Accessions & $\mathrm{bp} /$ residues \\
\hline complete & 5,168 & genomic & $1,510,537$ & $102,394,850,748$ \\
\hline fungi & 99 & RNA & $1,382,728$ & $2,276,250,402$ \\
\hline invertebrate & 353 & protein & $5,482,876$ & $1,870,214,220$ \\
\hline microbial & 1,494 & \multirow{9}{*}{\multicolumn{3}{|c|}{ Abbreviations: \#, number; bp, base pair }} \\
\hline mitochondrion & 1,384 & & & \\
\hline plant & 126 & & & \\
\hline plasmid & 634 & & & \\
\hline plastid & 131 & & & \\
\hline protozoa & 87 & & & \\
\hline vertebrate (mammalian) & 247 & & & \\
\hline vertebrate (other) & 664 & & & \\
\hline viral & 2,060 & & & \\
\hline
\end{tabular}

\subsubsection{UCSC Genome Browser}

The University of California Santa Cruz (UCSC, http://genome.ucsc.edu) has created a variety of tools, including a graphical tool for viewing a specified region of a genome and a collection of aligned annotations (77-81). The database for the UCSC Genome Browser provides integrated sequence and annotation data for a large collection of vertebrate and model organism genomes, including human, mouse, rat, and fruit fly (Table 2.3). Each sequence in the UCSC Genome Brower database includes assembly data, comparative genome annotations, and mRNA, EST and RefSeq gene alignments from GenBank. The browser is updated whenever a revised genome is released. Beginning in 2006, UCSC publishes an annual article with information on the latest database, available tools for viewing and comparing genes and new features (77-81). 
Table 2.3: Available Sequence and Annotation Downloads in UCSC Genome

\section{Browser}

\begin{tabular}{l|l}
\hline $\begin{array}{l}\text { Verterbrates } \\
\text { (Complete } \\
\text { Annotation Sets) }\end{array}$ & $\begin{array}{l}\text { human, cat, chicken, chimpanzee, cow, dog, fugu, guinea pig, } \\
\text { horse, lamprey, marmoset, medaka, mouse, opossum, } \\
\text { orangutan, platypus, rat, rhesus, stickleback, tetraodon, } X . \\
\text { tropicalis (western clawed frog), zebrafish }\end{array}$ \\
\hline $\begin{array}{l}\text { Verterbrates } \\
\text { (Sequences } \\
\text { downloads only) }\end{array}$ & $\begin{array}{l}\text { armadillo, bushbaby, elephant, european hedgehog, rabbit, } \\
\text { shrew, tenrec, tree shrew }\end{array}$ \\
\hline Deuterostomes & $\begin{array}{l}\text { C. } \text { intestinalis (sea squirt), lancelet, S. purpuratus (purple } \\
\text { urchin) }\end{array}$ \\
\hline Insects & $\begin{array}{l}\text { A. gambiae (African malaria mosquito), A. mellifera (western } \\
\text { honey bee), D. ananassae, D. erecta, D. grimshawi, D. } \\
\text { melanogaster (fruit fly), D. mojavensis, D. persimilis, D. } \\
\text { pseudoobscura, D. sechellia, D. simulans, D. virilis, D. } \\
\text { willistoni, } D . \text { yakuba, T. castaneum (red flour beetle) }\end{array}$ \\
\hline Nematodes & $\begin{array}{l}\text { C. brenneri (small nematode), C. briggsae (small nematode), } \\
\text { C. elegans (nematode), C. remanei (worm), P. pacificus } \\
\text { (roundworm) }\end{array}$ \\
\hline Other genomes & $\begin{array}{l}\text { S. cerevisiae (baker's yeast), SARS } \\
\text { 13 }\end{array}$ \\
\hline
\end{tabular}

\subsubsection{Data Information}

For this study, EST sequences were downloaded from GenBank/dbEST (72) at NCBI. The latest downloadable version was NCBI-GenBank Flat File Release 165.0 dated 4-15-2008 (http://www.ncbi.nlm.nih.gov/dbEST/). The mRNA sequences were downloaded from RefSeq (76) at NCBI. The latest release was NCBI Reference Sequence (RefSeq) Database Release 29 dated 5-4-2008

(http://www.ncbi.nlm.nih.gov/RefSeq/). It contained 5,168 organisms and 8,376,141 entries, including 1,510,537 genomic sequences (102,394,850,748 bases), 1,382,728

\footnotetext{
${ }^{13}$ SARS, or severe acute respiratory syndrome, is a severe form of pneumonia which appeared in outbreaks in 2003. It is transmitted especially by contact with infectious material (as respiratory droplets or body fluids), and is characterized by fever, headache, body aches, a dry cough, hypoxia and usually pneumonia.
} 
RNAs $(2,276,250,402$ bases), and 5,482,876 protein sequences $(1,870,214,220$ bases). Human and rat genomes were downloaded from the UCSC Genome Browser (http://genome.ucsc.edu/) (79). The latest release for the human genome was hg18 in 52004. The latest version of the rat genome was rn4 dated 11-2004.

\subsection{Data Format}

Sequences retrieved from the NCBI website can be displayed in several formats. These include ASN.1 (abstract syntax notation one) (82), FASTA (83), XML (extensible markup language) (84), and GenBank Flat File (85). Researchers can choose the specific format in order to receive the information they need. The two most common formats are GenBank and FASTA.

When a sequence is submitted to NCBI, it is recorded in the GenBank Flat File Format $(85 ; 86)$. The basic information for this format includes locus, definition, accession, version, keywords, source (a sub-category includes the organism), reference (sub-categories include authors, title, journal, medline, and pubmed), comment, features (sub-categories include source, coding region (CDS), gene), base count, and origin. Figure 2.1 shows a representative GenBank file for GenInfo Identifier (GI) 1392161.

SOURCE and LOCUS can be used to determine from which organism the sequences are derived and the size of the sequences. For a detailed description of each item involved in a GenBank file format, please refer to $(85 ; 86)$. 


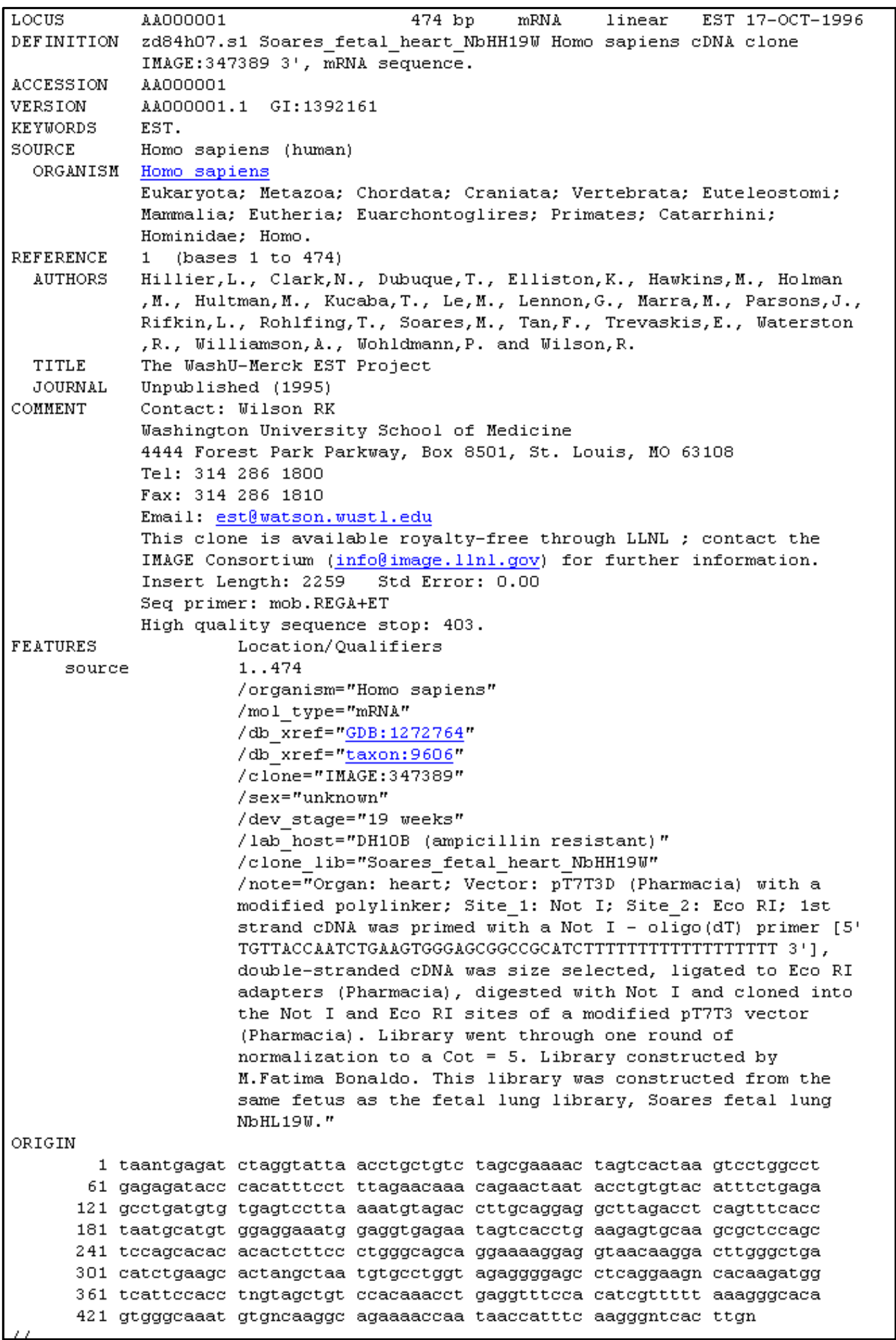

Figure 2.1: Example GenBank file 1392161. 
A sequence in FASTA data format (83) begins with a greater-than symbol '>', followed by single line description. In strict FASTA format, the data includes the GI number, locus name, source organism, gene name/protein name, and molecular type. The symbol ' $\mid$ ' is used to separate each piece information. The next few lines are sequence data, the combination of A, C, G and T nucleic acids or twenty different amino acids for that GI number. An example of the FASTA format for GI number 1392161 is shown in Figure 2.2. In a text file containing more than one sequence, the greater-than '>' symbol can be used to determine the beginning of the next sequence.

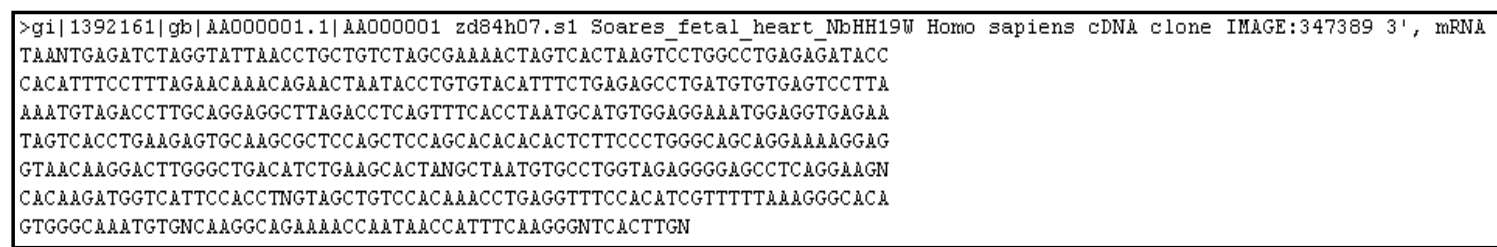

Figure 2.2: Example FASTA format 1392161.

GenBank and FASTA file formats both provided the necessary information for this research project. However, FASTA files are much smaller than files in GenBank format due to the exclusion of various annotations. Once the appropriate data was downloaded, it was preprocessed for use in each project. Section 2.3 covers the necessary steps in data preprocessing. 


\subsection{Preprocessing Data}

After the appropriate data was downloaded, it needed to be preprocessed. This involved filtering sequences by organism, masking repetitive elements and locating low complexity regions.

\subsubsection{Filtering by Organism}

Sequences in dbEST are submitted by researchers all over the world and organized into files according to the time they were received. Therefore, several different organisms may be represented in each of the dbEST files (release 165.0 included 713 files). Since the project examined ESTs on an organism-by-organism basis, the sequences had to be reorganized by organism before they could be analyzed.

Each sequence from the downloaded files was stored in GenBank flat file format (85). To re-organize the sequences by organism, the SOURCE field, which shows the scientific name of the organism, was used. A Perl script was written in order to read through the SOURCE field and assigned sequences to files according to organism.

A GenBank flat file format contains more annotation than is necessary for the purpose of sequence comparison. To align and compare two sequences, only the identifier and sequence data are needed. Therefore, a program called gb2fasta in the WU BLAST software package was used to convert the sequences into FASTA data format (83). After this conversion, the size of a sequence file was greatly condensed by removing annotations. 


\subsubsection{Masking Repetitive Elements}

Raw ESTs which have been organized by organisms are not ready for sequence mapping and clustering. Repetitive elements are sequences that appear more than once in a genome. Most genomes contain repetitive elements. For instance, over $40 \%$ of the human genome is composed of repetitive elements $(42 ; 44)$. The presence of such sequences can severely lengthen the search time. Thus, these regions should be removed or masked from consideration within raw EST files.

RepeatMasker is a software package that was designed to screen DNA sequences for repeats $(87 ; 88)$. RepeatMasker compares input sequences to a library of repetitive elements including interspersed and internal repeats. For this study, repetitive elements were masked by hard masking, which replaces each of the nucleotides in a repetitive region with 'N'. Soft masking can also be used to replace the DNA characters with their lower case equivalent. The computational time for sequence comparisons is decreased after the repetitive elements are masked (57).

\subsubsection{Filtering Low Complexity Regions}

Low complexity regions are those "regions of biased composition including homopolymeric runs, short-period repeats, and more subtle overrepresentation of one or a few residues" (89). They occur in both nucleotide and protein sequences. A low complexity sequence is a sequence with a biased nucleotide or amino acid composition that differs significantly from the general composition observed in either the sequences or a dataset of sequences. 
Masking these regions leaves biologically relevant regions available for matching and increases computational speed. The DUST (70) and SEG (70;90) programs are designed for this purpose. DUST is used especially for masking or filtering lowcomplexity regions in nucleic acids and SEG targets in amino acids. The low-complexity nucleic acid regions are masked with N's and amino acid regions are masked with X's. 


\section{CHAPTER III}

\section{COMPUTATIONAL SEQUENCE ALIGNMENT}

There are many methods available for finding the percentage of similarity between two sequences. The two main types of alignment are global and local. Global alignment refers to aligning the entire length of two sequences. Local alignment looks for significant similarities in smaller sections of the sequences.

An exhaustive algorithm will search through all possible alignments to find the best match between two sequences. However, such an approach requires intensive computation. Even if the sequences are only 10 bases long each, there are $O\left(2^{10} \times 2^{10}\right)=$ $O\left(2^{10+10}\right)=O\left(2^{20}\right)=1,048,576 \sim 1 \mathrm{M}$ comparisons. Therefore, more efficient approaches are needed and used.

\subsection{Dynamic Programming Algorithm (DPA)}

Dynamic programming is a method of solving the problems when the solutions can be determined recursively by building upon known solutions of a subspace. The term dynamic programming was first used by Richard Bellman in the 1940s (91). This type of problem has the properties of overlapping subproblems and optimal substructure. A famous example is finding the $n$th member of the Fibonacci sequence (See Equation 3.1). Later results are based on previous calculation.

$$
f i b(n)=\left\{\begin{array}{ll}
0 & \text {, if } n=0 \\
1 & \text {, if } n=1 \\
f i b(n-1)+f i b(n-2) & \text {, if } n \geq 2
\end{array} \quad\right. \text { (Equation 3.1) }
$$


Dynamic programming can be used in sequence alignment by combining solutions from aligning sequence prefixes which are computed once and saved in a matrix (92). There are three steps in a basic DPA: matrix initialization, matrix scoring fill and traceback. A DPA can locate the minimal matching or mismatching bases and edit the distance between two strings by inserting or deleting gaps. DPA has an $O(m \times n)$ time and space complexity where $m$ and $n$ are the size of the strings.

Global alignment requires an alignment of one entire string $X$ to a second entire string Y. Gaps may be inserted in either or both sequences to achieve the best result. The Needleman-Wunsch algorithm, which was proposed in 1970 by Needleman and Wunsch, is the most famous algorithm used to create a global alignment. It was the first instance of dynamic programming being applied to biological sequence comparison and it is guaranteed to find the alignment with the maximum score, given a scoring scheme (93).

Local alignment finds matching pairs of segments, each a part of a longer sequence, which have significant similarity. The Smith-Waterman algorithm, created in 1981, is one of the most famous algorithms used in local alignment (94).

In 1983, Ukkonen suggested a method to reduce computational complexity by adding an additional matrix in order to restrict the search space in the main matrix to a linear approach (95). In 1986, Myers used this approach and reduced the time to $O(n d)$ time in the worst case and $O\left(n+d^{2}\right)$ on average, where $n$ is the length of the longer string of the two strings and $d$ is the Hamming distance ${ }^{14}$ between the sequences. In addition, a penalty cost is included to deal with the gaps between two sequences $(94 ; 96)$.

\footnotetext{
${ }^{14}$ If two sequences of length $N$ differ from each other at $n$ sites, then the proportion of differences, $n / N$, is referred as the Hamming distance or the degree of divergence.
} 


\subsection{Heuristic Algorithm}

DPA will produce the optimal alignment for sequence comparisons. However, DPA is still computationally complex in terms of space and time. Therefore, a heuristic algorithm which "can make an approximation of the best solution without exhaustively considering every possible outcome (89)" like FASTA and BLAST, can reduce time and space complexity in calculation.

\subsubsection{FASTA}

FASTA stands for FAST-ALL. Developed by Pearson and Lipman, it combines local searching with a heuristic algorithm (97) to search a database for sequence homology. FASTA compares protein or nucleotide sequences to report the best matches of sequences for local alignment.

The FASTA program first generates a lookup table for matching sequence patterns of amino acids or nucleotides in a query sequence to sequences in a database without gaps. A parameter ktup is used, with a word size of 3-6 for nucleotide sequences and 1-2 for amino acid sequences, to screen the database for the pattern and insert matches into the lookup table. A scoring matrix is then used to determine the ten best aligned regions. Third, gaps are inserted between the high-scoring regions to join them together. Finally, a Smith-Waterman algorithm is used to align the highest scoring sequences. The optimal alignments of the query sequences to the best database matches will be printed as output (98-100). 


\subsubsection{BLAST}

BLAST stands for Basic Local Alignment Search Tool. It is the most widely used software package for rapid DNA and protein sequence comparison. It can be used to detect high scoring areas of local similarity between a query sequence and a target database of sequences.

BLAST searches sequences one-by-one in the database. If the current sequence in the database shares similarity to the query, BLAST returns the result to the user. Otherwise, BLAST will search the next sequence in the database.

The initial filter of the BLAST algorithm searches for seed sequences of a particular length, 11 bases for NCBI nucleotide-nucleotide BLAST, with 100\% conservation between the target and query sequences. Initial hits of seed sequences are then extended to check for larger regions of similarity. Through these searches, BLAST makes it possible to look for sequence homology within sequences by performing database searches, motif searches, and gene identification for both DNA and protein sequences.

There are two versions of BLAST used widely in research. One is free, the version produced by the National Center of Biotechnology Information (NCBI), called NCBI BLAST. The other requires an academic license agreement. This is the version created by Washington University (WU) in Saint Louis, Missouri, called WU BLAST $(69 ; 70)$. 


\subsubsection{Current BLAST Tools}

The BLAST software was first published in 1990 by researchers at the National Center of Biotechnology Information (NCBI). In the nearly 18 years since its original development, a number of changes have been made. As mentioned previously, the most widely used versions of BLAST are NCBI BLAST and WU BLAST. Both versions of BLAST offer a suite of programs. These programs perform nucleotide-nucleotide comparison (blastn), protein-protein comparison (blastp), nucleotide-protein comparison (tblastn), and translated nucleotide-nucleotide comparison (tblastx).

In addition, the NCBI suite offers two programs for looking at patterns gathered from multiple alignments of sequence conservation as seeds into sequence alignment. These two programs are position specific iterated BLAST (PSI-BLAST) and pattern hit initiated BLAST (PHI-BLAST) (101;102).

Speed is a problem in searching large quantities of DNA and protein sequences, even with heuristic algorithms such as BLAST. However, BLAST has qualities that lend it to being parallelizable.

\subsection{4 mpiBLAST}

An open source of parallelization of BLAST was developed as mpiBLAST by the

Los Alamos National Laboratory and released at the High Performance Computing Revolution 2003. This tool attempts to reduce disk I/O in a single machine and parallelize NCBI BLAST on PC clusters.

mpiBLAST splits a large database into smaller fragments and performs the master-worker scheme of job running. Through the technique of message passing 
interface (MPI) $(103 ; 104)$, mpiBLAST sends out sequence database fragments to different nodes. Each node individually performs the computation of NCBI BLAST on the query sequence and assigned fragment of original database. When all requested nodes finish their sub-tasks, the master node combines the results and send it back to the job sender $(105 ; 106)$.

\subsection{EST to Genome Alignment Algorithms}

Several programs align ESTs to genome sequences. Each of them has its own purpose. For example, sim4 is designed to quickly align a cDNA sequence to its parent sequence with high accuracy (107) while Spidey aligns cDNA sequences without considering the length of introns (108). Compared to more commonly used tools like BLAST, these programs incorporate more specific goals of distinguishing the boundaries of exons and introns.

Specific nucleotide pairs, such as the canonical CT/AG and GT/AG (109-113), are usually found at exon/intron boundaries. est2genome, Spidey, sim4, GMAP and EXALIN can help to find the positions of exon/intron boundaries, for fine tuning the alignments.

\subsection{1 est2genome}

est2genome is a tool used to predict genes by finding sequence homology. It implements a linear space dynamic programming algorithm to compare spliced sequences with their genomic counterparts on both the forward strands and reverse complement. est2genome will report a list of the exons and introns and indicate the predicted gene transcription (114). 


\subsubsection{Spidey}

Spidey was designed in 2001 to accomplish two main goals: "finding good alignments regardless of intron size" and "avoiding getting confused by nearby pseudogenes and paralogs" (108). It uses BLAST and a DPA type dot-plotting method (115) called DotView to align a single genomic sequence to a group of mRNA sequences. The hits resulting from these local alignment tools are sorted and assigned into different windows. Another round of BLAST is performed with the entire mRNA sequences in each window. A summary of the alignment is reported.

\subsection{3 $\operatorname{sim} 4$}

The program sim4 aligns a cDNA sequence with another genomic sequence rapidly and accurately (107;116). It starts with a BLAST-like computation to find highscoring pairs (HSPs) of aligned regions between two sequences. Next, sim4 uses DPA to choose a set of HSPs which might represent a gene in increasing order. sim4 allows for introns in the calculation, but the introns must be distinguishable in the results. Therefore, sim4 determines the boundaries of exons and computes the alignments (107).

\subsubsection{GMAP}

GMAP stands for Genomic Mapping and Alignment Program. It aligns mRNA and EST sequences to genomic sequences. Before processing GMAP, the data is entered into an indexed database file. Then GMAP finds and reports the location of an EST, aligns the EST to genomic sequences, and provides an integer interval tree to store the look up information associated with a given cDNA's position in the genome. It can map 
and align a cDNA over multiple genomic sequences at the same time. However, GMAP does not provide statistical analysis of alignments and therefore users cannot filter results based on statistical significance (117).

\subsubsection{EXALIN}

EXALIN, which was released in 2006, compares sequences one by one. Its approach is based on a novel method for searching spliced alignment, comparing sequence similarity with that obtained from position specific scoring matrix (PSSM) splice site models. In order to maximize the power of discrimination in sequence alignment and comparison, the scoring systems are chosen based on genomes. Currently, the scoring matrices and splice site models for human, mouse, rice and Caenorhabditis elegans are available. Dynamic programming was implemented in the tool to find the optimal computational result. Although dynamic programming returns the best result in alignment, it is slow in computation. Therefore, input output from blastn as an optional choice in EXALIN would help speed up the comparison (118).

\subsection{Case Study in Comparison of Current BLAST Software}

Sections 3.2.2 to 3.2.4 described how BLAST works. NCBI BLAST, WU BLAST and mpiBLAST all have a level of parallelization built in. The default setting of NCBI BLAST is to run on a one processor using multithreads. WU BLAST runs two threads ${ }^{15}$ at the same time in one processor machine. mpiBLAST requires at least two nodes, but can run on an unlimited number depending on the number of processors available.

\footnotetext{
${ }^{15} \mathrm{~A}$ thread is a portion of a program that can run independently of and concurrently with other portions of the program.
} 
Theoretically, searching for sequence similarities in a parallel fashion on multiple machines should be faster than using a single processor on the same data set. With this pre-assumption, mpiBLAST should be the fastest of the three BLAST programs.

As discussed in Section 1.10, ESTs are used to detect novel isoforms and tissue specificity for alternative splicing events. In addition, ESTs are used to identify the possible locations of pseudogenes in chromosomal sequences. All of these processes start with alignment of ESTs to the genome. Therefore, a study was conducted to compare the efficiency of each version of BLAST software: ncbiBLAST, wuBLAST and mpiBLAST. Finding the most efficient BLAST algorithm would speed up the initial alignment of ESTs to genome and reduce the computational time of the following projects.

\subsection{Methods}

\subsubsection{Data}

Comparisons were made by focusing on human EST and genomic data. Thirteen representative human EST sequence files and five representative chromosome sequence files were chosen (Table 3.1). Each sequence file contained data for multiple sequences. These files were chosen based on the total number of sequences in the file, the total number of nucleotide bases in the file and the average sequence length to ensure that they were representative snapshots of the entire human EST and genomic data set. 
Table 3.1: Data Used for BLAST Comparison

\begin{tabular}{llrrr}
\hline Nequence & File Size & $\begin{array}{c}\text { No. of } \\
\text { Sequences }\end{array}$ & \multicolumn{1}{c}{$\begin{array}{c}\text { No. of } \\
\text { bases }\end{array}$} \\
\hline Genome & chr14 & $88,935,052$ & 1 & $87,191,216$ \\
& chr22 & $35,039,241$ & 11 & $34,352,072$ \\
& chr17 & $80,964,851$ & 30 & $79,346,966$ \\
& chr9 & $120,146,880$ & 60 & $117,790,386$ \\
chr1 & $231,366,553$ & 91 & $226,828,929$ \\
\hline EST & est283 & 3,350 & 7 & 2,593 \\
& est277 & 7,259 & 15 & 5,781 \\
& est136 & 300,995 & 521 & 210,054 \\
& est147 & 724,214 & 1,085 & 563,737 \\
& est149 & $1,603,440$ & 2,433 & $1,244,897$ \\
est126 & $2,466,032$ & 3,031 & $2,053,889$ \\
est241 & $3,312,648$ & 4,991 & $2,502,256$ \\
est176 & $7,002,704$ & 10,149 & $5,274,323$ \\
& est113 & $19,434,153$ & 20,347 & $16,714,000$ \\
est270 & $16,447,012$ & 24,764 & $12,934,924$ \\
est1 & $19,969,430$ & 36,800 & $14,021,420$ \\
est91 & $33,883,614$ & 50,522 & $27,683,871$ \\
est166 & $33,064,286$ & 71,748 & $25,130,960$ \\
\hline
\end{tabular}

Abbreviations: chr, chromosome; No., number.

Chr1 represents chromosome 1; est1 represents dbEST file 1.

Table adapted from Table 1 in (119).

EST sequence data was downloaded using the GenBank Flat File Release 141.0, dated 4-15-2004 (72). The resulting 305 dbEST files were parsed to include only those with Homo sapiens as the organism. After the human ESTs were identified, they were masked for repetitive elements using RepeatMasker $(87 ; 88)$.

The repeat masked human goldenpath genomic assembly hg16-Jul2003 ${ }^{16}$, dated 7-2003, was downloaded for use from UCSC Genome Browser (http://genome.ucsc.edu)

\footnotetext{
${ }^{16}$ The genomic sequences in goldenpath genomic assembly hg16-Jul2003 version were split in pieces in most chromosome in 2003 because the sequences were still under debugging process. In addition, the technique to save contents in a text file has its upper limit in file size. The latest version of hg18-Mar2006 has merged most contigs from the same chromosome into one complete sequence. Those pieces that are not clear defined are placed in the random sequence. Therefore, for most chromosomes in the Human Genome would have up to two sequences in file.
} 
(77-81). Each contig ${ }^{17}$ for a particular chromosome was placed into a file based on its cytogenetic position as an individual sequence entry. Blastable databases for each chromosome were created using the appropriate tool (xdformat for wuBLAST; formatdb for ncbiBLAST; and mpiformatdb for mpiBLAST) $(92 ; 106)$.

For mpiBLAST, the database was also segmented into fragments using the command mpiformatdb. Each fragment might not contain the same number of sequences, but should be nearly equal in overall size. In order to determine the number of fragments to use in mpiBLAST, three different sequences, chr22, chr1 and est166 were examined. The number of fragments, and therefore nodes used, was determined according to the resulting fragment size. The results are shown in Table 3.2.

Table 3.2: Fragment Information in mpiBLAST

\begin{tabular}{c|cc}
\hline sequence & No. of fragments & size per fragment \\
\hline chr22 & 9 & $\sim 1 \mathrm{MB}$ \\
& 8 & $\sim 2 \mathrm{MB}$ \\
& 5 & $\sim 4 \mathrm{MB}$ \\
& 3 & $\sim 9 \mathrm{MB}$ \\
& 1 & $\sim 35 \mathrm{MB}$ \\
\hline chr1 & 44 & $\sim 2 \mathrm{MB}$ \\
& 30 & $\sim 5 \mathrm{MB}$ \\
& 25 & $\sim 8 \mathrm{MB}$ \\
& 20 & $\sim 11 \mathrm{MB}$ \\
& 15 & $\sim 19 \mathrm{MB}$ \\
& 10 & $\sim 19 \mathrm{MB}$ \\
& 1 & $\sim 227 \mathrm{MB}$ \\
\hline est166 & 26 & $\sim 1 \mathrm{MB}$ \\
& 13 & $\sim 2 \mathrm{MB}$ \\
& 7 & $\sim 4 \mathrm{MB}$ \\
& 1 & $\sim 26 \mathrm{MB}$ \\
\hline
\end{tabular}

Abbreviations: chr, chromosome; No., number Table adapted from Table 2 in (119).

\footnotetext{
${ }^{17}$ A contig is a continuous sequence of DNA created by the assembly of overlapping sequenced fragments of a chromosome. It is also a group clones representing overlapping regions of a genome.
} 


\subsubsection{Types of Sequence Searches}

The factors affecting speed are the size of the database, the size of the query, the number of database sequences and the number of query sequences. Types of sequences searches can be:

- A single, relative short sequence (such as a single EST) vs. a larger database;

- A larger database of smaller sequences (with different lengths) vs. a large database of smaller sequences;

- A large database of smaller sequences vs. a large database of large sequences;

- A large database of large sequences vs. a large database of larger sequences.

In order to determine the efficiency of the various versions of BLAST, the representative EST and genomic data were used in four separate comparisons between different target database (T) and query sequences $(\mathrm{Q})$ : EST (T) vs. EST (Q); chromosome (T) vs. chromosome (Q); chromosome (T) vs. EST (Q); and EST (T) vs. chromosome (Q).

EST vs. EST compared the computational efficiency when sequences in both the database and query are short. Chromosome vs. chromosome tested for situations when both query sequences and target databases are large sequences. Compared to an EST sequence, which contains only 300-600 bp on average, the number of nucleotide bases in a chromosome contig is much larger. However the number of sequences in each chromosome file is smaller, ranging from one sequence (chr14) to 91 sequences (chr1) for the representative sequences. Therefore, in chromosome vs. EST, the longer (in base length) sequences, or ESTs, were used as a database and the smaller sequences were used as the query. In the fourth comparison, we reversed the database and query sequences as 
EST vs. chromosome in order to determine if the order of comparison had an impact on computational results.

\subsubsection{System and Software}

The comparisons were based on the current version of the appropriate BLAST software available at the time of analysis. ncbiBLAST 2.2.9 (release date 5/12/2004) was downloaded from NCBI (http://www.ncbi.nlm.nih.gov/BLAST/). For wuBLAST, an academically licensed version 2.0MP WashU (release date 8/22/2004) was downloaded from Washington University BLAST Archives (http://blast.wustl.edu). mpiBLAST 1.2.1 (release date 2/6/2004) was downloaded from mpiBLAST ${ }^{18}$.

In order to test the parallel versions, a 16-node (32 CPU) Microway Athlon MP GigaCluster ${ }^{\mathrm{TM}}$ with an additional master node was used. Each node on the cluster has a dual AMD Athlon ${ }^{\mathrm{TM}}$ MP 2400+ 384KB cache, 512 MB DDR, 266 MHz ECC/REG low profile, plus a 40GB hard drive. Red Hat Linux version 7.3 (120) with MPICH $(103 ; 104)$, Portable Batch System (PBS) (121), and Microway Cluster Management Software were installed. wuBLAST, ncbiBLAST and mpiBLAST were run on three different systems. Each job was sent and managed by PBS.

wuBLAST and ncbiBLAST were also compared using two identical PCs. These PCs were equipped with a dual AMD Athlon ${ }^{\mathrm{TM}}$ MP processor $2800+512 \mathrm{~KB}$ cache. These machines also had a 640KB system RAM, a 2048 Extended RAM and a 120GB mass drive as a storage system. The operating system was Suse Linux version 9.1 (122), 2.6.4-52-smp i686 machine, and i386 hardware platform.

\footnotetext{
${ }^{18}$ The mpiBLAST website used for this study was hosted by the Los Alamos National Laboratory (http://mpiblast.lanl.gov) at the time the project was developed. Since then two of mpiBLAST's main authors moved to Virginia Tech, the mpiBLAST website has been changed to http://www.mpiblast.org/.
} 


\subsection{Results}

\subsubsection{EST vs. EST}

The first series of comparisons involved short length sequence database versus short length sequence queries. All thirteen representative EST sequence files (Table 3.1) were placed into individual blastable databases. Each database was aligned to the thirteen representative EST sequences files one at a time.

The test revealed that when the number of sequences in the database is small, ncbiBLAST seems to perform marginally better than wuBLAST (Table 3.3). The results indicated a delay of up to $\sim 690 \mathrm{sec}(\sim 11.5 \mathrm{~min})$ for wuBLAST on the data used.

However, as the number of sequences in the database increased, the processing time for wuBLAST tended to be smaller in comparison to ncbiBLAST (Table 3.4). This is especially evident in the four comparisons of the last two sequences, est91 vs. est91, est91 vs. est166, est166 vs. est91, and est166 vs. est166, where it took wuBLAST 3,699 7,702 seconds (61.2 128 minutes) to complete the work while it took ncbiBLAST 8,557 14,312 seconds (142.6 238.5 minutes), or approximately twice as long, to do the same job.

It took more time complete each task for est270, which contained 24,764 sequences, especially using wuBLAST. When est270 served as a query, it required more time than the other three longer sequences: est1, est91, and est166. When est270 was used as both the query and database, it took 12,822 seconds (237 minutes) to perform the comparison. This made the cumulative time to complete the whole series higher than any other series in Figure 3.1. 
Table 3.3 ncbiBLAST Performs Best when the Number of Sequences in the Database is Small (Measured in sec)

\begin{tabular}{|c|c|c|c|c|c|c|c|c|c|c|c|c|c|c|c|}
\hline \multirow[b]{3}{*}{ query } & \multirow{3}{*}{$\begin{array}{c}\text { \# of } \\
\text { sequence }\end{array}$} & \multicolumn{14}{|c|}{ database } \\
\hline & & \multicolumn{2}{|c|}{ est283 } & \multicolumn{2}{|c|}{ est277 } & \multicolumn{2}{|c|}{ est136 } & \multicolumn{2}{|c|}{ est147 } & \multicolumn{2}{|c|}{ est149 } & \multicolumn{2}{|c|}{ est126 } & \multicolumn{2}{|c|}{ est241 } \\
\hline & & wu & ncbi & wu & ncbi & wu & ncbi & wu & ncbi & wu & ncbi & wu & ncbi & wu & ncbi \\
\hline est283 & 7 & 0 & 0 & 0 & 0 & 0 & 0 & 0 & 0 & 0 & 0 & 0 & 0 & 0 & 0 \\
\hline est277 & 15 & 0 & 0 & 0 & 0 & 0 & 0 & 0 & 0 & 0 & 0 & 0 & 0 & 0 & 1 \\
\hline est136 & 521 & 10 & 5 & 10 & 5 & 15 & 10 & 16 & 11 & 18 & 14 & 22 & 14 & 21 & 18 \\
\hline est147 & 1,085 & 21 & 10 & 21 & 12 & 27 & 20 & 35 & 28 & 41 & 47 & 41 & 35 & 52 & 70 \\
\hline est149 & 2,433 & 47 & 23 & 47 & 25 & 59 & 44 & 71 & 62 & 98 & 118 & 88 & 77 & 120 & 175 \\
\hline est126 & 3,031 & 59 & 30 & 60 & 33 & 77 & 58 & 82 & 67 & 104 & 87 & 132 & 115 & 124 & 135 \\
\hline est241 & 4,991 & 96 & 48 & 97 & 53 & 116 & 91 & 142 & 127 & 199 & 232 & 176 & 162 & 244 & 364 \\
\hline est176 & 10,149 & 197 & 98 & 197 & 108 & 262 & 189 & 299 & 236 & 387 & 402 & 414 & 321 & 460 & 573 \\
\hline est113 & 20,347 & 395 & 212 & 402 & 226 & 492 & 384 & 535 & 425 & 629 & 505 & 771 & 686 & 725 & 742 \\
\hline est270 & 24,764 & 472 & 241 & 485 & 259 & 749 & 436 & 758 & 496 & 965 & 691 & 1430 & 769 & 1302 & 947 \\
\hline est1 & 36,800 & 693 & 344 & 698 & 369 & 834 & 615 & 910 & 709 & 1071 & 975 & 1238 & 1014 & 1341 & 1325 \\
\hline est91 & 50,522 & 959 & 495 & 970 & 527 & 1242 & 888 & 1253 & 1025 & 1435 & 1263 & 1697 & 1544 & 1820 & 1777 \\
\hline est166 & 71,748 & 1338 & 670 & 1394 & 707 & 1587 & 1149 & 1690 & 1372 & 1897 & 1760 & 2189 & 2057 & 2312 & 2373 \\
\hline
\end{tabular}


Table 3.4: wuBLAST Performs Best when the Number of Sequences in the Databases is Greater (Measured in sec)

\begin{tabular}{|c|c|c|c|c|c|c|c|c|c|c|c|c|c|}
\hline \multirow[b]{3}{*}{ query } & \multirow{3}{*}{$\begin{array}{c}\text { \# of } \\
\text { sequence }\end{array}$} & \multicolumn{12}{|c|}{ database } \\
\hline & & \multicolumn{2}{|c|}{ est176 } & \multicolumn{2}{|c|}{ est113 } & \multicolumn{2}{|c|}{ est270 } & \multicolumn{2}{|c|}{ est1 } & \multicolumn{2}{|c|}{ est91 } & \multicolumn{2}{|c|}{ est166 } \\
\hline & & wu & ncbi & wu & ncbi & wu & ncbi & wu & ncbi & wu & ncbi & wu & ncbi \\
\hline est283 & 7 & 1 & 1 & 1 & 1 & 1 & 1 & 1 & 1 & 1 & 2 & 1 & 2 \\
\hline est277 & 15 & 1 & 1 & 1 & 1 & 1 & 1 & 1 & 2 & 2 & 2 & 2 & 3 \\
\hline est136 & 521 & 43 & 28 & 60 & 43 & 175 & 44 & 66 & 50 & 88 & 74 & 63 & 74 \\
\hline est147 & 1,085 & 79 & 100 & 99 & 109 & 190 & 114 & 122 & 124 & 163 & 187 & 137 & 186 \\
\hline est149 & 2,433 & 168 & 242 & 212 & 234 & 385 & 252 & 247 & 277 & 337 & 417 & 295 & 406 \\
\hline est126 & 3,031 & 226 & 194 & 378 & 357 & 749 & 366 & 368 & 349 & 584 & 590 & 435 & 585 \\
\hline est241 & 4,991 & 329 & 496 & 430 & 488 & 756 & 526 & 502 & 574 & 676 & 860 & 601 & 847 \\
\hline est176 & 10,149 & 890 & 1021 & 1158 & 1014 & 3160 & 1067 & 1251 & 1136 & 1793 & 1749 & 1364 & 1754 \\
\hline est113 & 20,347 & 1207 & 1101 & 2860 & 2789 & 4064 & 2314 & 1980 & 2148 & 3729 & 4240 & 2642 & 3836 \\
\hline est270 & 24,764 & 3475 & 1451 & 3565 & 2834 & 12822 & 3384 & 5114 & 2614 & 6911 & 4214 & 4341 & 4236 \\
\hline est1 & 36,800 & 2078 & 2003 & 2785 & 3114 & 5307 & 3237 & 3446 & 3720 & 4203 & 5424 & 3781 & 5443 \\
\hline est91 & 50,522 & 2800 & 2607 & 4722 & 5021 & 7227 & 4846 & 4343 & 4928 & 3669 & 9882 & 5772 & 8557 \\
\hline est166 & 71,748 & 3109 & 3653 & 4857 & 6319 & 6978 & 6362 & 5316 & 6613 & 7727 & 11210 & 7702 & 14312 \\
\hline
\end{tabular}




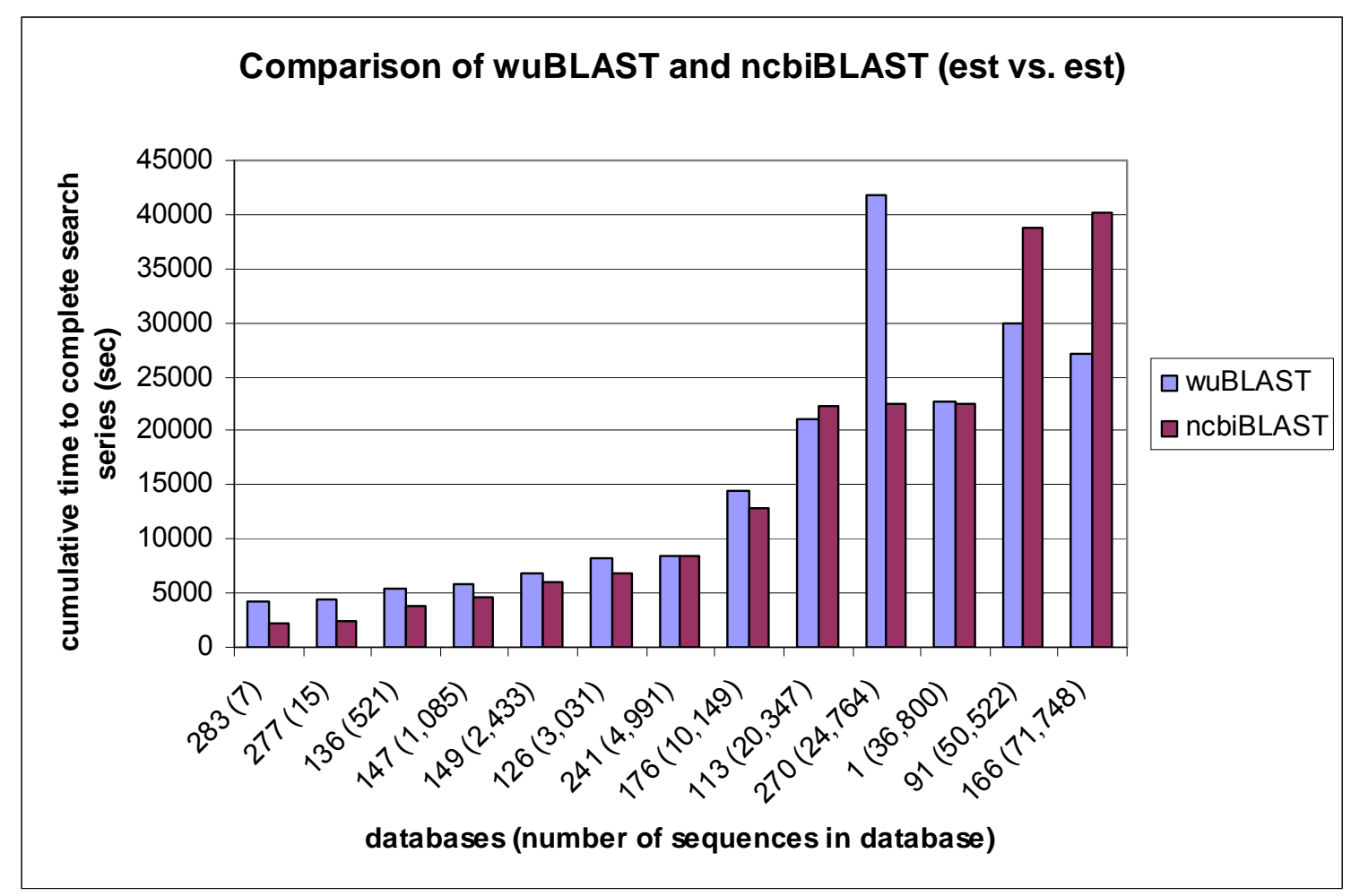

Figure 3.1: Cumulative results of wuBLAST and ncbiBLAST in EST vs. EST comparison.

(283: est283; 277: est277; and so on) Image adapted from Figure 2 in (119).

After scanning the sequences in the est270 file, one possible reason that it took longer to complete, with the longest time occurring in comparisons of est270 with itself in wuBLAST, is that there are poly-A tail sequences in the file. This kind of sequence can waste a lot of computation time. If a low-complexity filter, like DUST (70) or SEG (70;90), was utilized, this type of sequence could be masked or skipped, allowing the program to proceed to the next comparison. However, when the test was run, both wuBLAST and ncbiBLAST were operating by the default setting. In ncbiBLAST, lowcomplexity filter DUST is turned on by default for the blastn program and SEG is turned on by default for the blastp program. But in wuBLAST, users must add an additional command of "filter=dust" to activate the low-complexity filter. This 
would explain why wuBLAST took significantly more time than ncbiBLAST to process the est 270 vs. est 270 comparison. The wuBLAST program was attempting to compare the low-complexity sequences.

To run an mpiBLAST search, the minimum size of a database fragment is $1 \mathrm{MB}$. Therefore, the chosen EST sequence files under $1 \mathrm{MB}$ could not be tested in mpiBLAST because they could not be segmented into more than one database fragment.

The results for mpiBLAST using an EST vs. EST comparison are shown using est166 as the database, and four of the representative EST sequence files as the query (est136, est147, est241 and est176). All mpiBLAST searches were tested using 1-15 nodes, excluding the master node, due to the upper limitation of having 16 nodes on the system. The results indicated that as the number of nodes used to complete the search increase, the total search time decreased (Figures 3.2 and 3.3). However, mpiBLAST did not appear to be more efficient at searching for sequence similarities. 


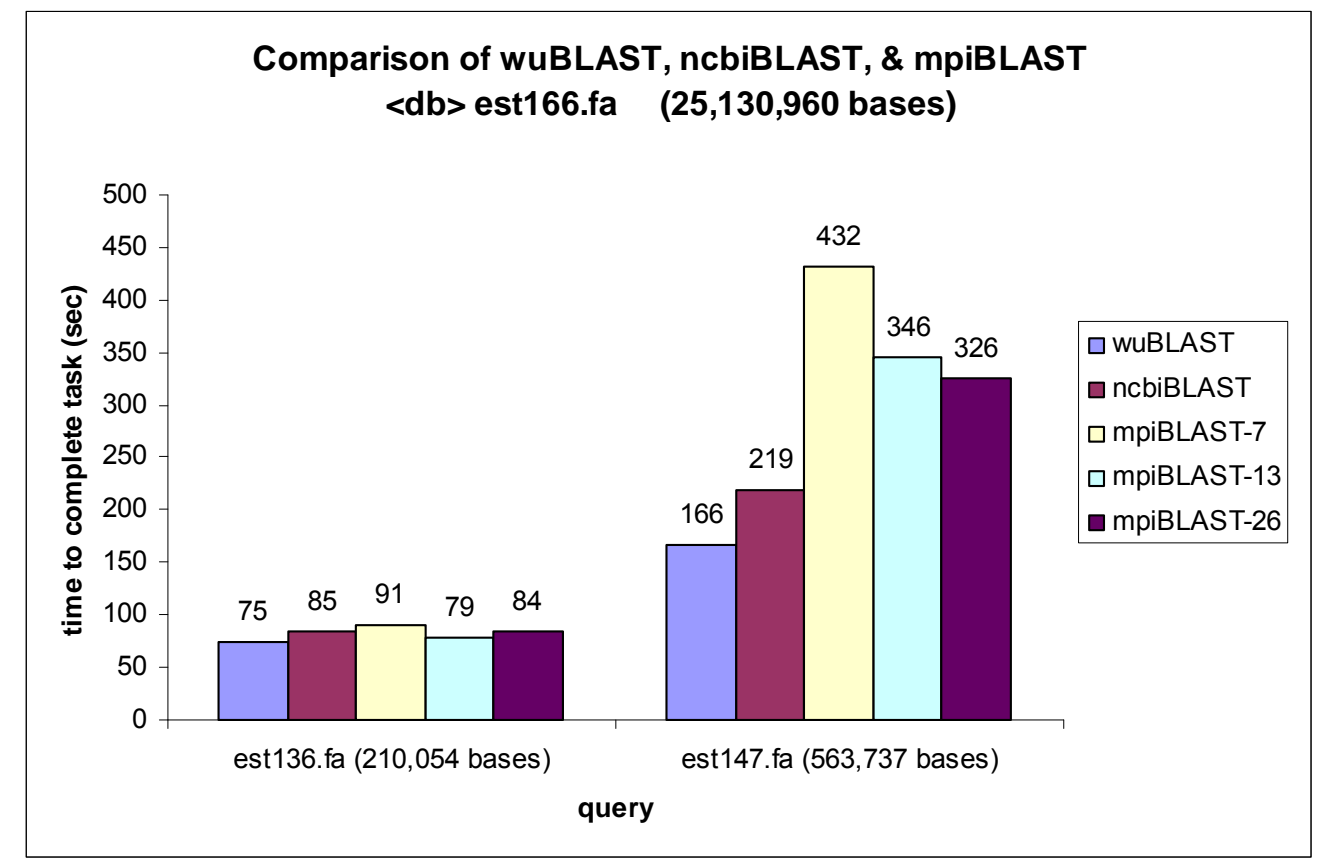

Figure 3.2: est166 target database vs. est136 and est147 query sequences. Image adapted from Figure $3 \mathrm{a}$ in (119).

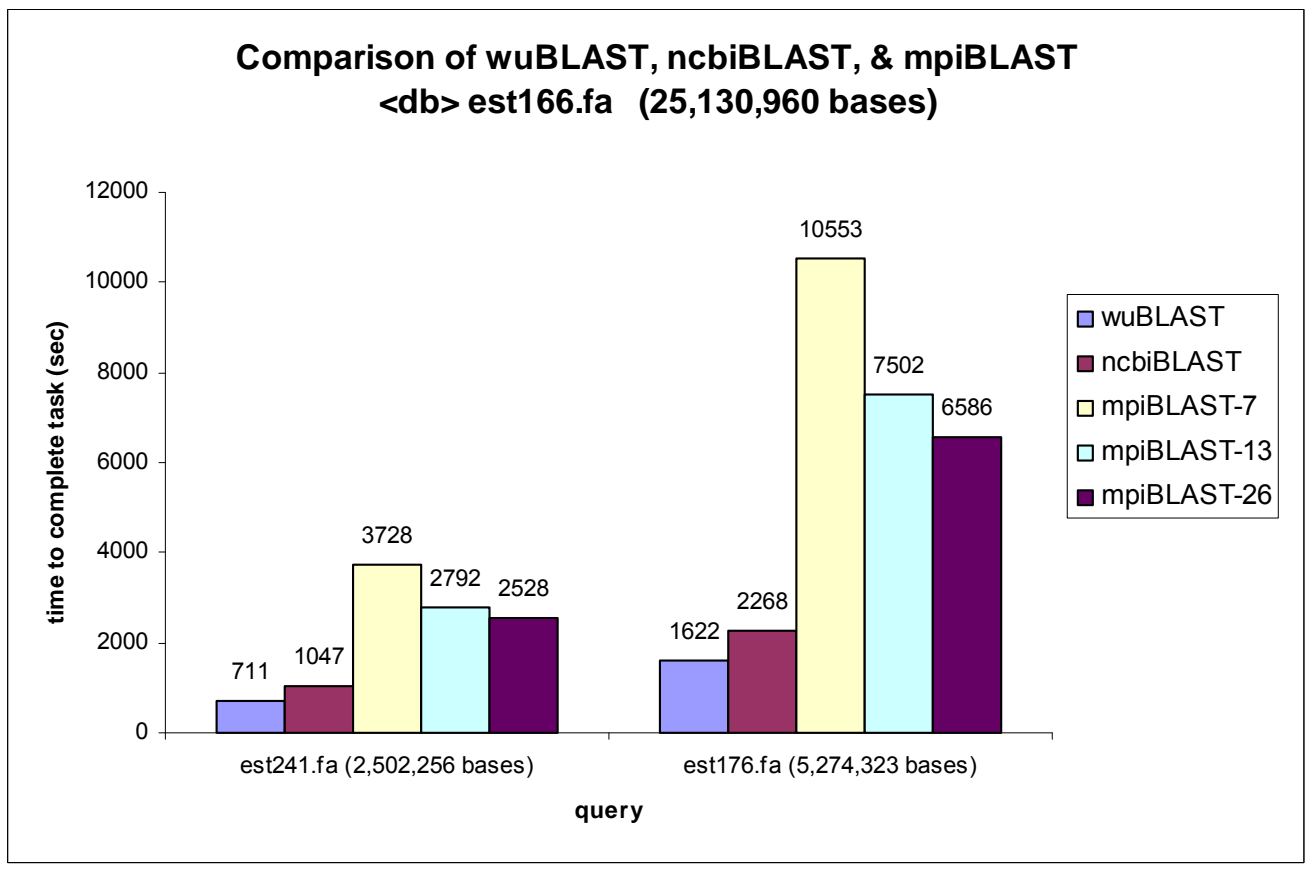

Figure 3.3: est166 target database vs. est241 and est176 query sequences. Image adapted from Figure $3 \mathrm{~b}$ in (119). 


\subsubsection{Chromosome vs. Chromosome}

It is possible the performance would vary drastically when both the target database and query contain a large number of bases per sequence due to a high memory load and increased disk I/O. In order to test this hypothesis, all five chromosome contig files were placed into individual blastable databases. Each database was compared to the five representative chromosome sequences separately.

Twenty-five comparisons were generated and the four cumulative results are presented in Figure 3.4. As the number of nucleotide bases, or size of the sequences increased, the cumulative time for both wuBLAST and ncbiBLAST to complete the series increased proportionally. In general, ncbiBLAST took 1.7 2.8 times longer to complete the series of work for each chromosome than wuBLAST. The cumulative time to complete the whole series of comparisons for chr1 as the database in wuBLAST was 53,301 seconds ( $\sim 14.8$ hours) (result not shown). It took almost a month to perform the same series of comparisons in ncbiBLAST. The reason for the tremendous increase in time in ncbiBLAST for chr1 is unknown, but could be related to a heavy memory load caused by the large number of sequences (91) and bases $(226,828,929)$. 


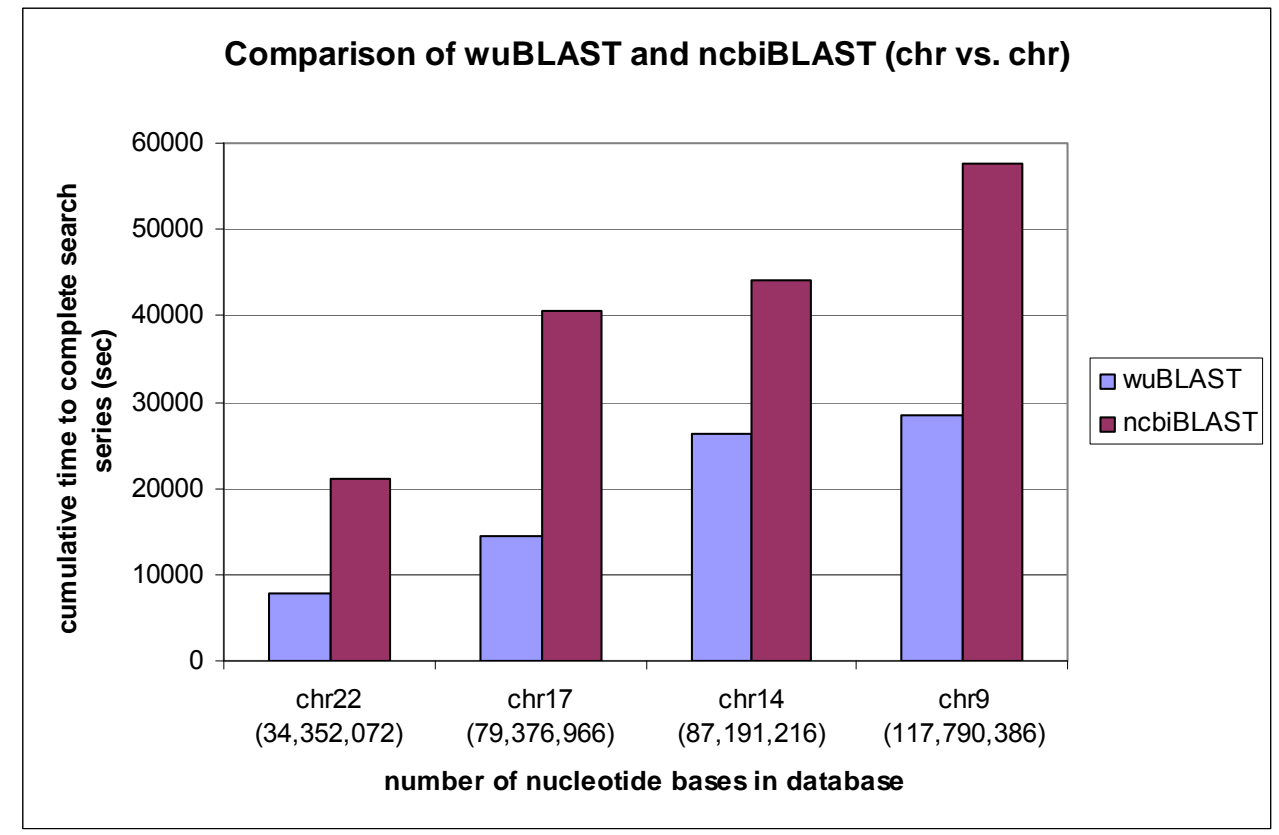

Figure 3.4: Cumulative results of wuBLAST and ncbiBLAST using chromosome vs. chromosome.

Image adapted from Figure 7 in (119).

\subsubsection{Chromosome vs. EST}

Comparing chromosome to EST uses larger sequences as target databases and smaller sequences as queries. The gain in efficiency of running mpiBLAST instead of ncbiBLAST appeared after using chromosome sequences as the database. The results for mpiBLAST were derived by using chr22 and chr1 as databases and two representative EST sequences, est136 and est147, as the queries. The results from wuBLAST, ncbiBLAST and mpiBLAST are shown in Figures 3.5 and 3.6, with a variable number of nodes used for mpiBLAST (as listed in the figure legend). 


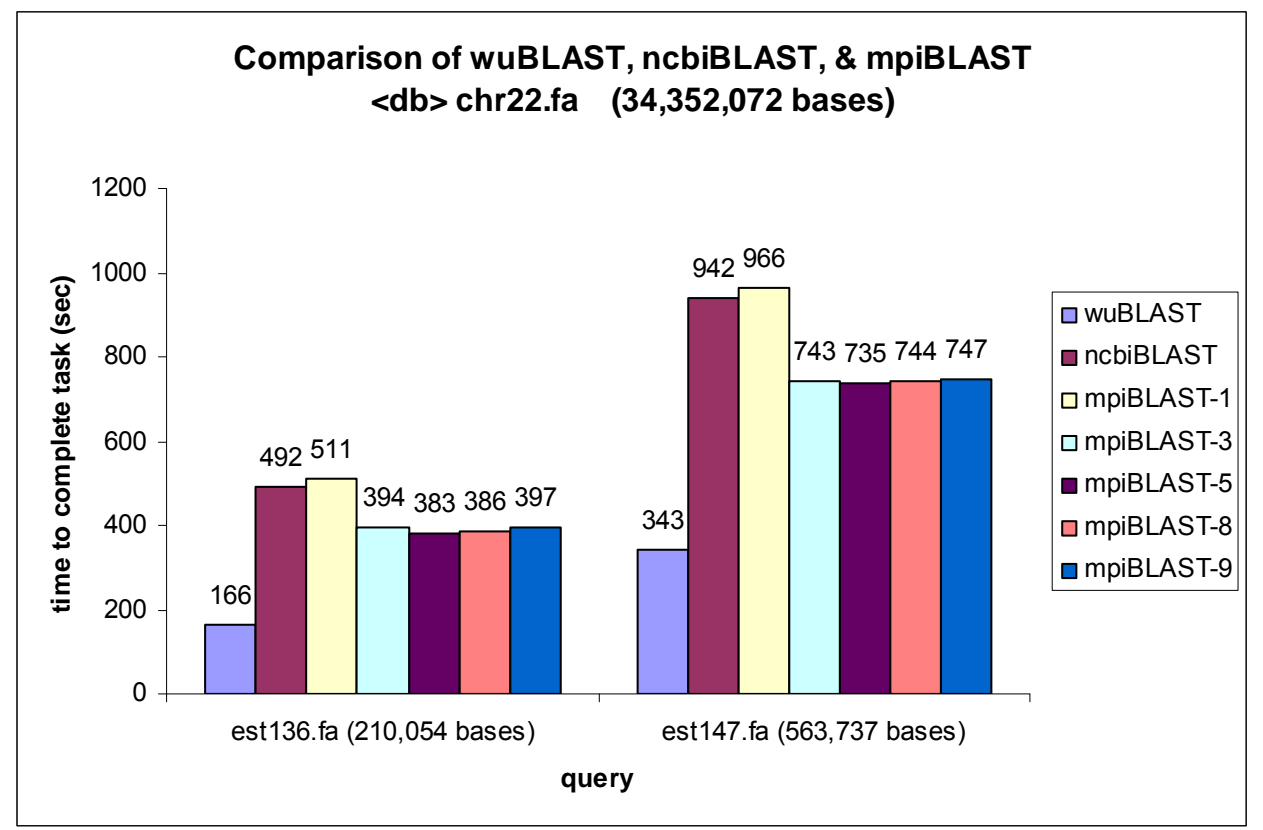

Figure 3.5: chr22 target database vs. est136 and est147 query sequences. Image adapted from Figure 6a in (119).

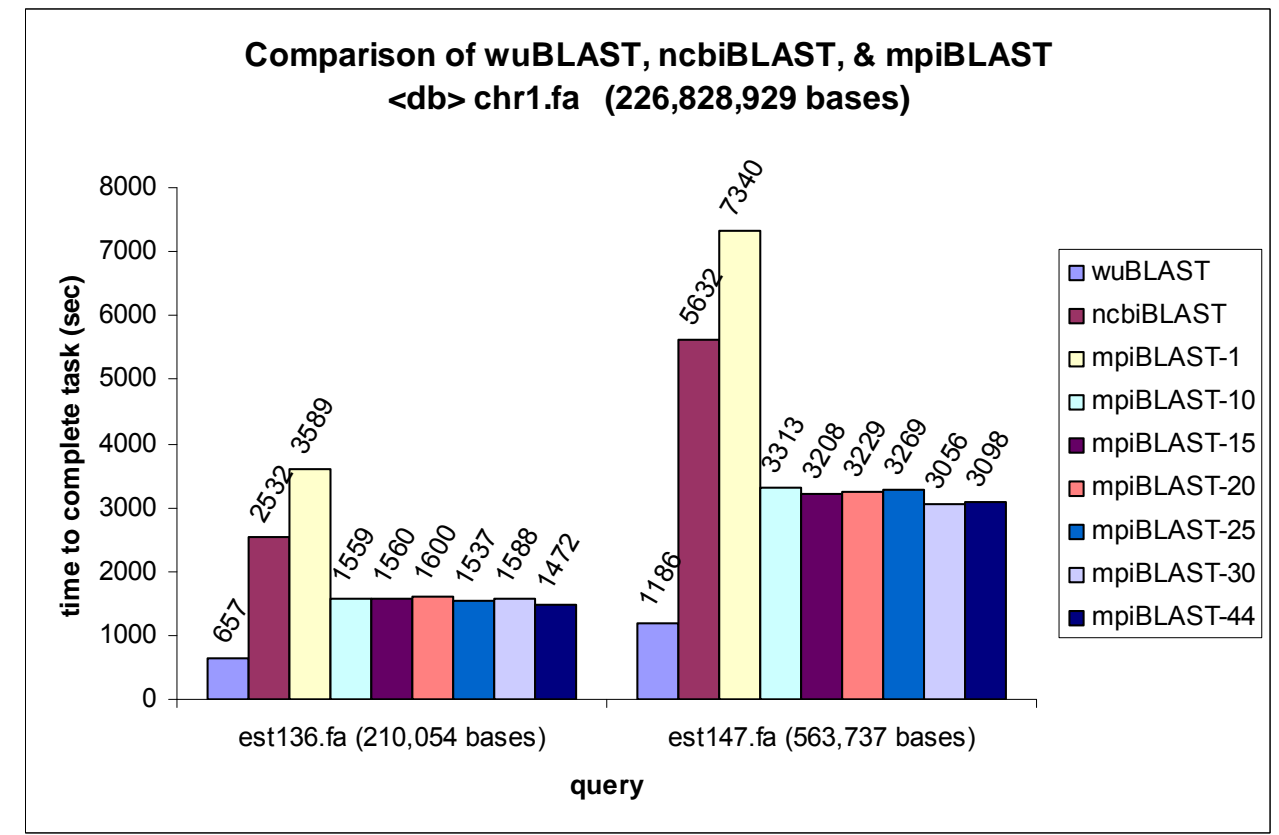

Figure 3.6: chr1 target database vs. est136 and est147 query sequences. Image adapted from Figure $6 \mathrm{~b}$ in (119). 
While mpiBLAST ran on multiple nodes in the cluster, wuBLAST and ncbiBLAST ran on a single node in the same cluster. The database is divided into $1,3,5,8$, and 9 segments for chr22 and 1,10,15,20, 25, 30 and 44 segments for chr1 (Table 3.2). These numbers are listed as mpiBLAST-number_of_segments. The number of nodes used to complete the task is listed in the parentheses.

wuBLAST worked the best. The speed of mpiBLAST increased when the

database was divided into more than 1 segment. However, if the database had only one segment, mpiBLAST performed slightly worse than ncbiBLAST due to the time it took for the master-worker scheme to deliver commands. It is worth noting that communication overhead for mpiBLAST has a significant impact on the database for searches with one segment.

\subsubsection{EST vs. Chromosome}

Comparisons using smaller sequences for the database and larger sequences as queries are unlikely in real world situations. However, in order to determine if the order in which these comparisons are made makes a difference on software performance, EST vs. chromosome was studied.

As in EST vs. EST, all thirteen representative EST sequence files were placed into individual blastable databases. Each database was searched for similarity to the five representative chromosome sequence files consecutively.

Each database search was performed as a series of five queries. Figure 3.7 shows the comparison between wuBLAST and ncbiBLAST as the cumulative time to finish each series of searches. 


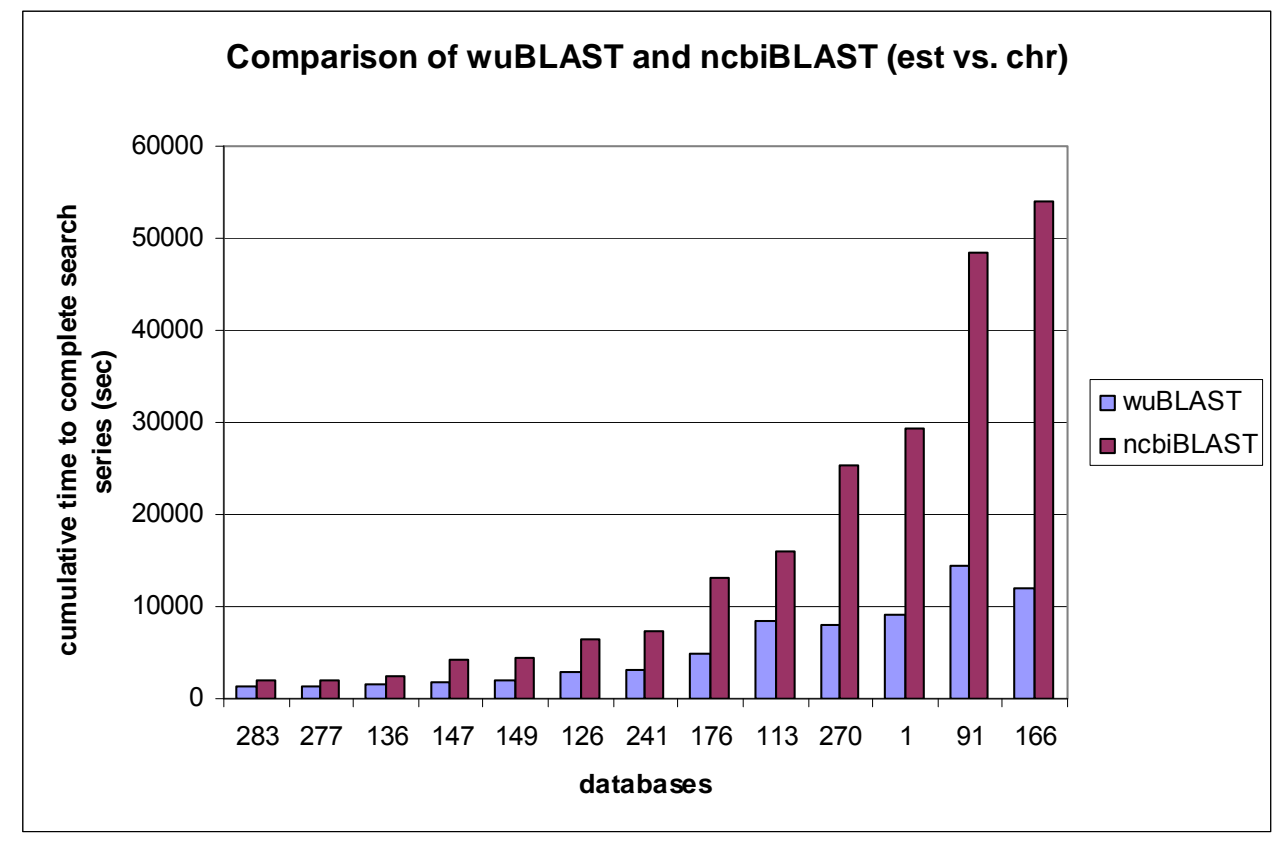

Figure 3.7: Cumulative results of wuBLAST and ncbiBLAST for EST vs. chromosome.

(283: est283; 277: est277; and so on) Image adapted from Figure 4 in (119).

Generally speaking, wuBLAST performed better than ncbiBLAST. ncbiBLAST took 1.6 4.5 times longer than wuBLAST to accomplish each series of comparisons.

The cumulative time taken by ncbiBLAST increased proportionally to the number of sequences in the database. This was not the case for wuBLAST. For instance, est 270 took less time for wuBLAST to process than est113. According to Table 3.1, the reason might be that est270 has more sequences $(24,764)$ but bases $(12,934,924)$ than est113 (20,347 sequences, $16,714,000 \mathrm{bp})$. Therefore the average number of bases per sequences is smaller. In another example, est166 was processed faster than est91. 


\subsection{5 mpiBLAST}

Figure 3.8 shows the results obtained by using different numbers of database fragments segmented for mpiBLAST with est166 as the database and est136 as the query. Theoretically, the relationship between the number of nodes and the speed of computation should be linear, as long as the number of fragments does not exceed the number of available processors. For example, for the thirteen fragments in Figure 3.8, the result of running on two nodes should be half of the result of running on one node. Likewise the result of running on three nodes should be one third of the result of running on one node, and so on. Thus, running thirteen nodes should produce the best result because there are thirteen fragments. However, as Figure 3.8 illustrates, dividing the database into seven fragments and running on three to four nodes provided the best result. If the database was divided into either thirteen or 26 fragments, the best results were obtained by running on six to seven nodes. For a single database fragment, the number of nodes did not make difference, as Figure 3.9 illustrates.

One notable exception is that the time for 26 fragments dropped when thirteen nodes were used in cluster. $26 \div 13=2$. The reason might be that equal number of fragments were routed to the worker nodes so that each node ran balanced jobs. Moreover, when the chromosome was used as the database, the optimal number changed (Figure 3.9). 


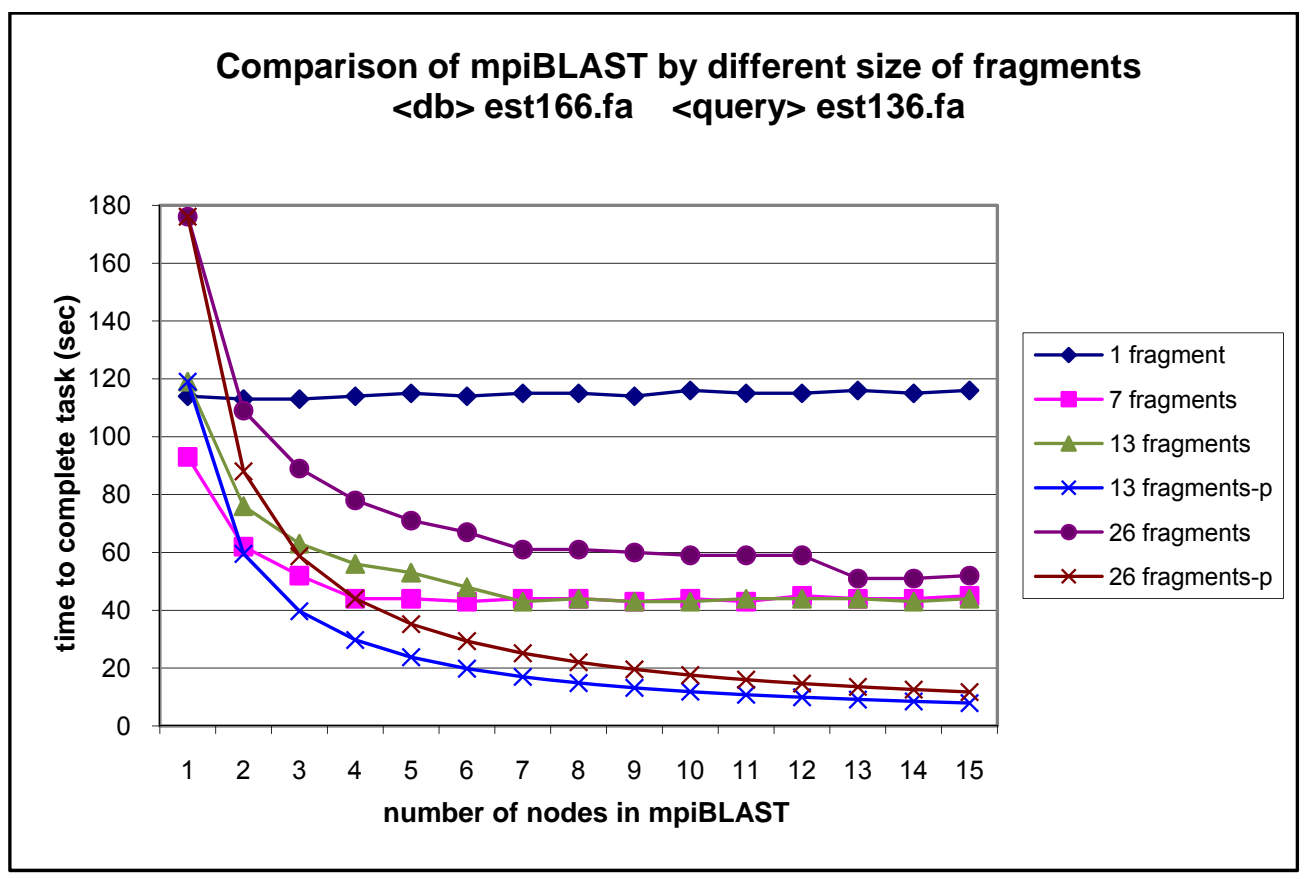

Figure 3.8: est166 target database vs. est136 query sequence in mpiBLAST. Image adapted from Figure 8a in (119).

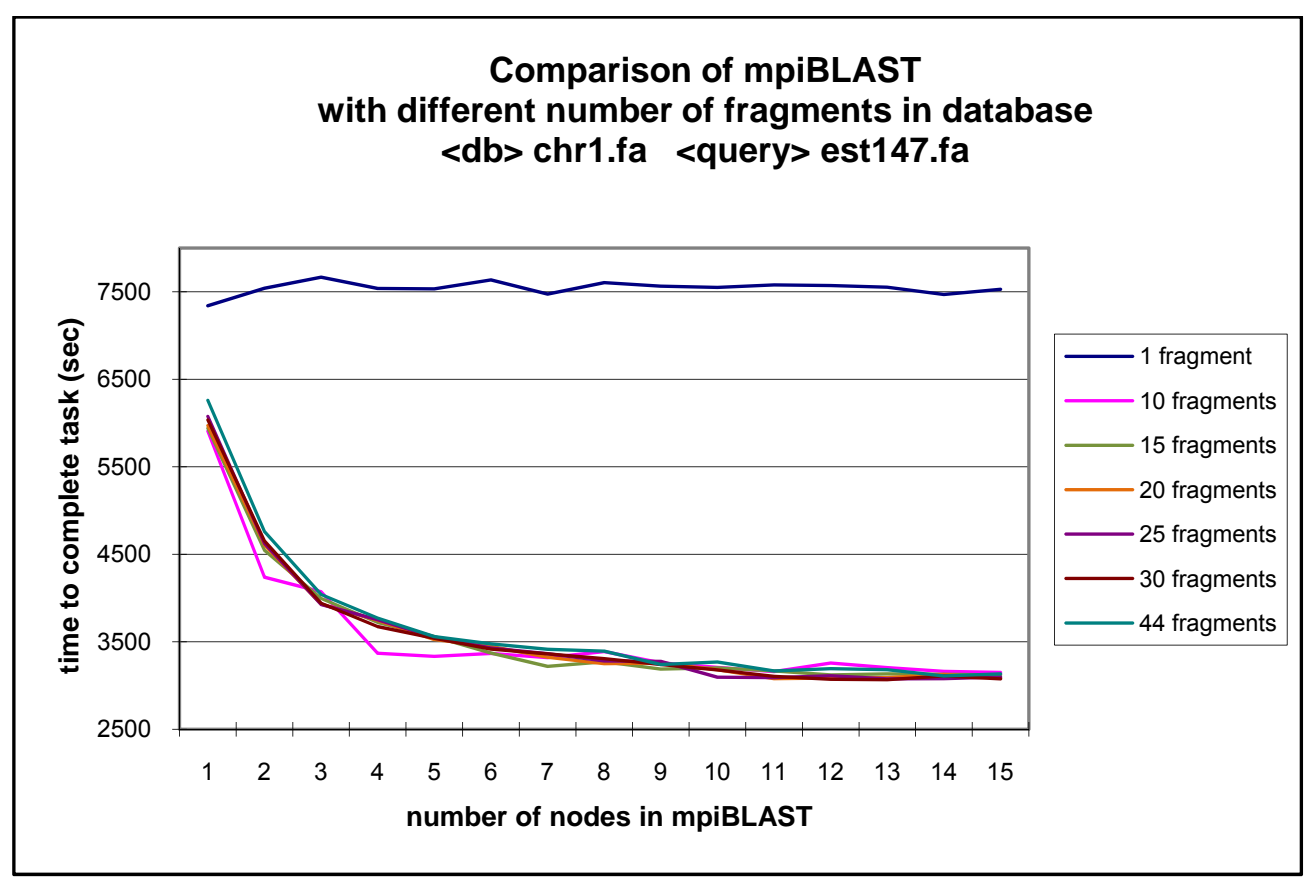

Figure 3.9: chr1 target database vs. est136 query sequence in mpiBLAST. Image adapted from Figure $8 \mathrm{~b}$ in (119). 


\subsection{Discussion}

As mentioned in Section 3.6.1, the comparisons were made using the default settings for the BLAST tools. In general, wuBLAST worked the best, if we do not consider the number of bases in a sequence and the size of the database. What causes wuBLAST to run faster is difficult to examine because it is not open source.

For biologists, ncbiBLAST is considered standard because it was the first of the BLAST tools developed for sequence comparison. However, this study suggests wuBLAST is worth the extra licensing fees and installation effort.

The average number of bases per sequence for each chromosome was much larger than the average number of bases per sequence for the ESTs. Hence, if both the size of a chromosome fragment and the size of an EST fragment was $\sim 1 \mathrm{MB}$, the chromosome fragment would contain fewer sequences with a larger average size than the EST fragment.

blastn compares sequences one by one between the database file and the queries. Fewer similarities between the database and query will result in a smaller time requirement for the comparison.

mpiBLAST attempts to decrease disk I/O. It performs better than ncbiBLAST because the content of each fragment can easily fit into cached memory. It avoids the allocation problem of large contents in memory causing more disk I/O. However, sending the database fragments to different nodes to complete the whole task required time for communication between the master node and slave/worker nodes. Figures 3.5, 3.6, 3.8 and 3.9 indicate where potential bottlenecks occurred. Another consideration is network 
flow. A network bottleneck could be a problem if lots of data needed to be transported at the same time.

In addition, breaking the database into pieces could affect the E-value. It would not be the same as the E-value calculated at once in any singular BLAST. Moreover, mpiBLAST itself does not change any I/O method to speed up its calculation. It first passes the database fragments to the slave/worker nodes and calls ncbiBLAST to run in parallel on those worker nodes.

\subsection{Conclusion}

To help identify any organism's transcriptome or transcribed portion of the genome, clustering a large number of sequences is necessary. It requires not only thousands of comparisons, but rather searching billions of records. Therefore, finding the best technique to compare sequences in parallel becomes paramount.

Based on the results, wuBLAST generally performs the best of the three tools using the default settings. However when dealing with a smaller number of sequences in the database, ncbiBLAST performs better. mpiBLAST decreases search time when the number of sequences in a fragment is small but the number of nucleotide bases is large. The user can choose a suitable BLAST based on the size of the data and the number of sequences. Note that these results are based on nucleotide sequences. There could be a different result in comparison of amino acids/protein sequences. 


\section{CHAPTER IV}

\section{COMPUTATIONAL METHOD FOR ALTERNATIVE SPLICE PATTERN RECOGNITION}

\subsection{Introduction}

\subsubsection{History of Alternative Splicing}

As discussed in Section 1.6, a DNA sequence is translated into a fully-functioning protein through transcription, pre-mRNA processing, translation and protein folding (Figure 4.1). During this process, the cellular machinery may splice out an exon or retain an intron in whole or in part before producing the final copy of mRNA transcript, resulting in the synthesis of a greater variety of proteins. This is called alternative splicing. Exons of the primary gene transcript are separated and then reconnected to produce an alternative ribonucleotide arrangement.

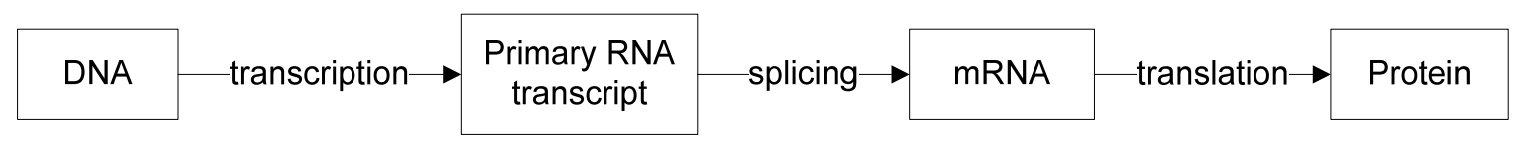

Figure 4.1: Central Dogma.

The mechanisms for alternative splicing were first uncovered during the late 1970s and early 1980s. In 1977, Phillip A. Sharp of the Massachusetts Institute of Technology (MIT) and Richard J. Roberts of New England Biolabs discovered that in the primary RNA transcripts, the non-coding regions - introns - were discarded for the final 
version of mRNA $(123 ; 124)$. Sharp and Roberts received a joint Nobel Prize in Medicine in 1993 "for their discoveries of split genes." In 1980, Randolph Wall of University of California Los Angeles (UCLA) showed that this basic view of pre-mRNA splicing, in which the introns are always discarded and all exons are always included in the mRNA, does not invariably hold true. In fact, the cellular machinery can "decide" to splice an exon or to leave an intron in the final mRNA transcript (125). In 1984, Tom Maniatis, Michael Green and their colleagues at Harvard University developed a test-tube procedure to reveal the molecular machinery performing the cutting of introns and pasting together of exons (126-128).

\subsubsection{Splicing Mechanism}

The biological mechanism which controls splicing was first described in 1984 as a splice machine now commonly known as a spliceosome (126). The spliceosome is a complex of five small nuclear uridine rich $\left(\operatorname{snRNA}^{19}\right)$ molecules labeled U1, U2, U4, U5 and U6 along with protein factors. Spliceosomes recognize exon/intron boundaries through a 5' donor site, a 3' acceptor site, a branch point and a polypyrimidine tract.

The canonical donor site recognized by the spliceosome complex is marked by the nucleotide GT, while the canonical 3' acceptor site is AG. There are other patterns confirmed within mammalian genomes including GC/AG, AT/AC, GT/GG and TT/AG $(129 ; 130)$. The canonical pattern GT/AG is predicted to occur $99.24 \%$ of the time. GC/AG occurs $0.69 \%$, AT/AC occurs $0.05 \%$, and the other patterns account for the remaining for $0.02 \%$ of all splice sites in mammals $(129 ; 130)$. The pattern AT/AC has

\footnotetext{
${ }^{19}$ A snRNA is a small RNA molecule that functions in the nucleus by guiding the assembly of macromolecular complexes on the target RNA to allow site-specific modifications or processing reactions to occur.
} 
been shown to result from a separate spliceosome complex involving U11 and U12 snRNA.

When canonical splicing begins, the U1 snRNA binds to the 5' end of the splice site and recruits several protein factors to form a commitment complex (131-133). This creates a branch site in which exons can attach at the donor's G nucleotide through a phosphodiester linkage. As part of the process, the U2 snRNA binds to the branch site. Afterwards, the U4-U5-U6 complex arrives and U6 replaces the U1. U1 and U4 disassociate from the complex. U2 and U6 link with the lariat intron that gets removed. U5 aids in merging the two adjoining exons together. Figure 4.2 shows the diagram of a canonical spliceosome.

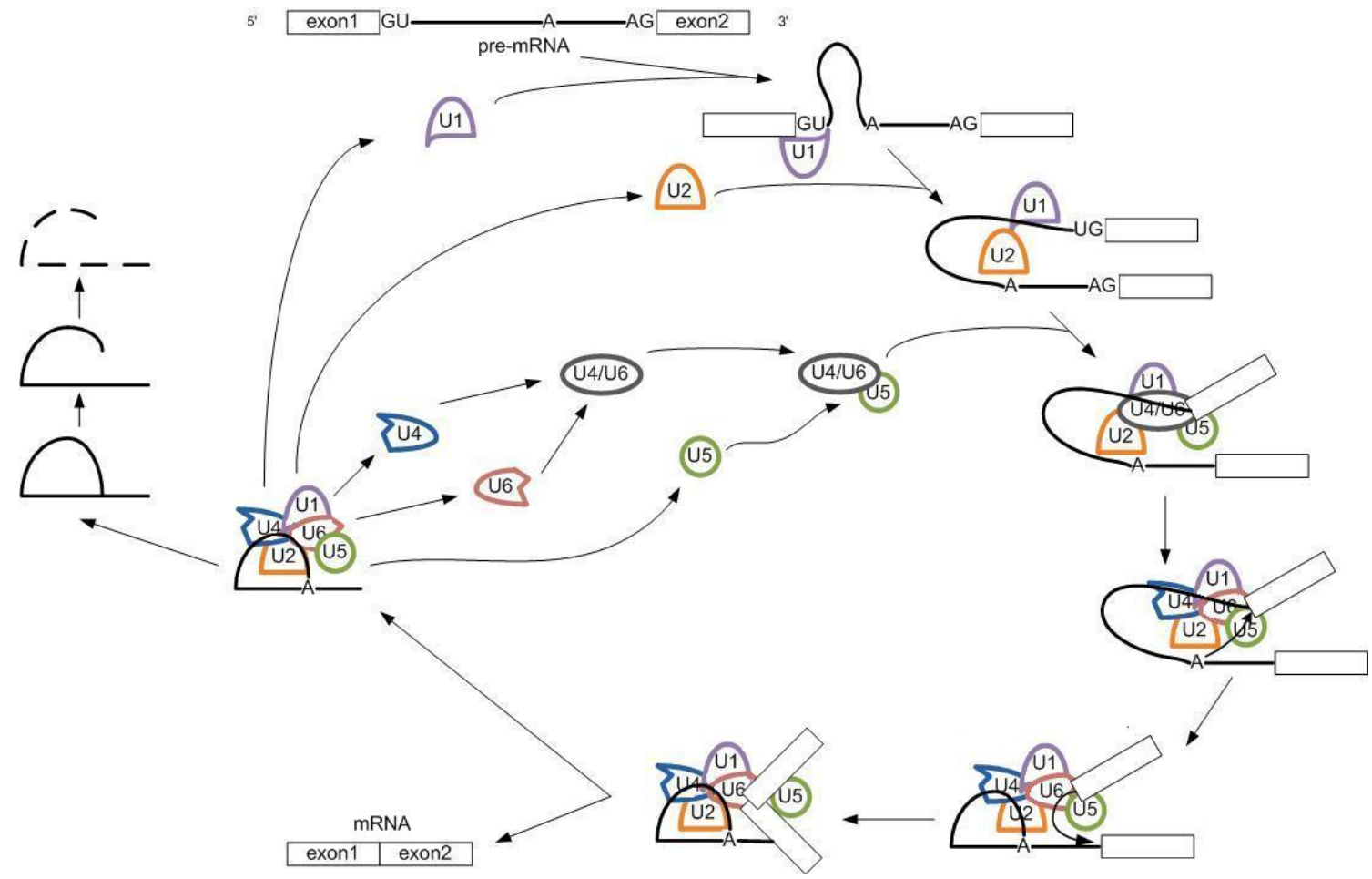

Figure 4.2: Canonical spliceosome for intron splicing. 


\subsubsection{Alternative Splicing Modes}

There are a number of alternative splicing events, including: alternative promoter selection, alternative polyadenylation sites, exon skipping, exon retention, exon extension, exon truncation and intron retention (Figure 4.3). Each of these is discussed in detail.

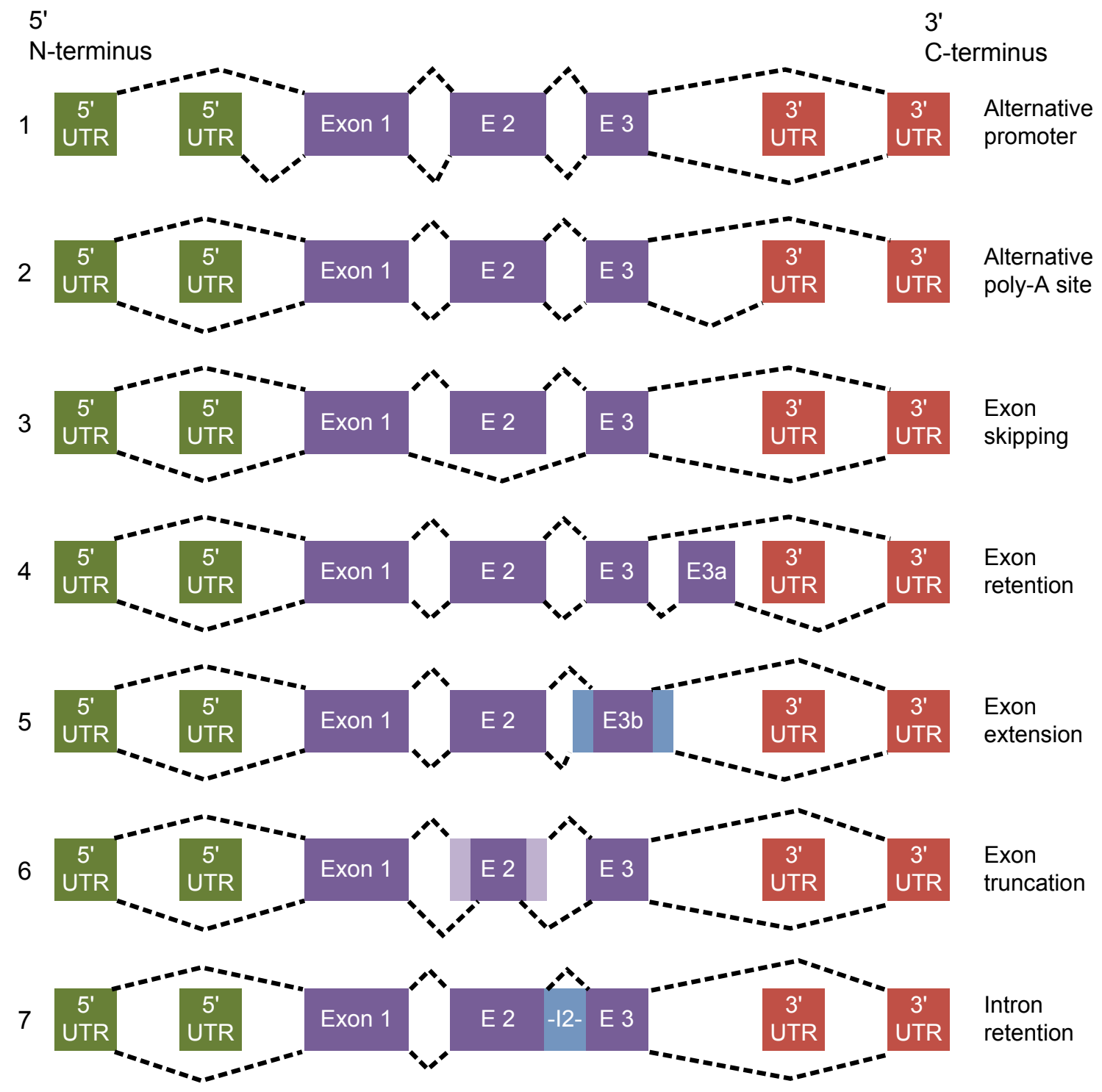

Figure 4.3: Alternative splicing events. 


\section{Alternative promoter selection}

In alternative promoter selection, alternative aminyl ends are produced in the processed mRNA by alternatively selecting promoter regions. These regions occur in the 5' untranslated region (UTR) of the mRNA. Such events do not alter the final protein product. This is the only method that can produce an alternative N-terminus domain in proteins. Different sets of promoters can splice certain sets of other exons.

\section{Alternative polyadenylation sites}

In some cases, the 3' UTR of the processed mRNA is alternatively spliced producing alternative polyadenylation sites. Once again, these events will not alter the translated protein product.

\section{Exon skipping}

This method of alternative splicing works by choosing certain exons to remove from the final processed mRNA. As a result, each exon can be thought of as a "cassette" that can potentially be removed. Exon skipping results in an altered translated proteins (134-136). This is the most commonly found version of alternative splicing in mammals.

\section{Exon retention}

In exon retention, additional exons located in the place that is normally an intronic region, are added to the final mRNA sequences, resulting in a different translated protein (137). 


\section{Exon extension}

In exon extension, sequences are added around a cassette exon at either the 5' or 3' ends. This results in a slightly modified mRNA sequence and produces a different protein $(134 ; 135 ; 138)$.

\section{Exon truncation}

Exon truncation occurs when a cassette exon is not fully added to an mRNA sequence. The 5' end, 3' end or both ends of the exon are removed, resulting an altered translated protein (137).

\section{Intron retention}

This method of alternative splicing occurs when an intron is retained in the final mRNA transcript $(134 ; 135 ; 138)$. As long as an expressible stop codon is not located in the intron, an altered translated protein is produced which is still functional. Intron retention is commonly found in plants and in lower multicellular organisms.

\subsubsection{Characteristics of Alternative Splicing}

Alternative splicing results in transcriptome/proteome complexity and gene function diversity. It is also highly relevant to human diseases. For instance, the breast cancer gene BRCA2 is a typical example of an exon skipping event (139). In addition, alternative splicing alters important protein features, such as phosphorylation, glycosylation, and transmembrane helices (140). 


\subsubsection{Rates of Alternative Splicing}

Initially, alternative splicing was thought to be an anomaly (125). In the early 1990s, it was predicted that about 5\% of all human genes provided alternatively spliced isoforms (124). By the late 1990s, researchers concluded that alternative splicing is much more prevalent than previously thought, with the potential for $50 \%$ of human genes to undergo alternative splicing $(141 ; 142)$. In recent studies, it has been found that up to $74 \%$ of human genes are alternatively spliced (143).

The average mammalian gene has between seven and eight exons (42). While this number seems small, this provides the possibility of over two hundred different cassette exon patterns. The largest number of known isoforms for a single coding region is the Drosophila DScam gene which has 38,016 distinct forms (144).

The ability of human genes to produce a number of different proteins helps explain the diversity among organisms that still have a relatively small percentage of variation in DNA. Among humans the similarity is measured to be around $99.5-99.8 \%$. This leaves a small difference of only $0.2-0.5 \%$. Yet this difference results in up to 15 million different nucleotides in humans (145).

\subsection{Research in Alternative Splicing Detection}

\subsubsection{Automatic Methods for Detecting Alternative Splicing}

Several research groups have used computational methods to analyze alternative splicing in the past decade. Some of them have studied ESTs through sequence alignment. Others have used different data sources, such as microarray data. 
Automatic methods which use ESTs to detect splicing variants generally go through several steps. First, mRNA sequences are aligned to genomic contigs ${ }^{20}$. Then, available ESTs are aligned to contigs. Next, sequences are clustered according to the genomic region where they are located. Finally, the alternative splices are defined as conflicting, contiguous, transitive or gapped. Most methods search for splice variants in a single organism. Other methods focus on detecting alternative splicing for a specific genome, such as the human or mouse genome. There are some methods that analyze splice variants by comparative analysis across species $(140 ; 142 ; 146)$.

There are other forms of analysis which do not use EST sequences as a reference for identifying alternative splicing. Researchers can also search for a set of possible exons and splice sites among all exon concatenations for the highest homology to a protein domain. Other methods analyze microarray data to monitor splicing events based on known spliced variants (147). The tiling microarray uses probes ${ }^{21}$ that cover the entire genome or contigs of the genome in an unbiased fashion $(148 ; 149)$. One method uses Pfam $^{22}$ information when clustering alternative splicing (150). Another process incorporates annotation integration resources (151).

\subsubsection{Research of Automatic Applications in Alternative Splicing Detection}

In the past decade, several papers have described different approaches for the automatic detection of alternative splicing. In 1999, Moronov used The Institute for

\footnotetext{
${ }^{20}$ A contig is a continuous sequence of DNA created by the assembly of overlapping fragments of a chromosome. The term also refers to a group of clones representing overlapping regions of a genome. ${ }^{21}$ A probe is a single-stranded DNA molecule used in laboratory experiments to detect the presence of complementary sequence among a mixture of other single-stranded DNA molecules.

${ }^{22} \mathrm{Pfam}$ (http://pfam.sanger.ac.uk) is a large collection of multiple sequence alignments and hidden Markov models covering many common protein domains and families. For each Pfam family, users are able to check for multiple alignments, view protein domain architectures, examine species distribution, connect to other databases, and study known protein structures.
} 
Genomic Research $\left(\mathrm{TIGR}^{23}\right)$ Human Gene Index as a reference for clustering the results after aligning ESTs to genomic DNA. The partial gene structures generated by spliced alignment were merged whenever they shared consecutive splicing sites spanning an intron. The superstructures corresponded to all possible gene structures for which each complete exon was supported by at least one of the alignments (142).

In 2000, Brett and his colleagues aligned ESTs to mRNA to identify insertions and deletions. Through analyzing the data, they identified alternative splicing genes (141). In the same year, Croft and his team created an intron database based on GenBank release 111. They identified ESTs that matched region previously only designated as intronic. With further examination, they found many unrecognized alternatively spliced exons, especially those in regions conserved between species (152).

In 2001, Kan and his colleagues aligned ESTs and genomic sequences to create transcripts for gene prediction. They elucidated gene structure as a complex problem that incorporated the splice junctions, gene boundaries, and alternative splicing. In addition, they analyzed shared regions of alternative splicing between the human and mouse genomes (146). In the same year, Modrek et al. aligned ESTs to genomic sequences using UniGene cluster information to detect alternative splices $(137 ; 153)$.

Lee and his group displayed alternative splicing graphically in 2003. Users can choose a cluster annotation and view splice variants within the same cluster (154). Johnson et al. further created a survey of exon junction microarrays based on known data (147).

\footnotetext{
${ }^{23}$ TIGR was a non-profit genomics research institute founded in 1992 by Craig Venter in Rockville, Maryland. It is now part of the J. Craig Venter Institute (http://www.tigr.org).
} 
In 2004, Sorek's group distinguished alternative splices from constitutive exons (155). The next year, Hiller et al. used Pfam information to analyze alternative splicing events (150). Florea incorporated annotation integration resources for analysis in alternative splicing (151). Chen and his colleagues compared data across human, mouse and rat genomes the following year (140). Castrignano et al. established a database resource called ASPicDB for alternative splicing analysis in 2008. They updated their database based on NCBI Entrez gene and UniGene databases (156).

\subsection{Applications for Alternative Splicing}

Research in alternative splicing helps to identify genes and define the functional roles of genes. It also serves to analyze the complexity of alternative splices, particularly on the proteome. Moreover, it reveals the regulation of alternative splice forms across the whole genome and recognizes different terms of genes. Alternative splices can help to differentiate tissue specificities. Comparative genomics studies the evolution of alternative splicing. Recently, alternative splicing events have been studied in relation to diseases.

\subsection{Case Study in Phosphodiesterase 4 (PDE4)}

In order to determine how ESTs can be used to detect isoforms and tissue specificity with alternative splicing, a case study of PDE4 in the rat genome was conducted. This was a joint work with Mr. Christopher M. Whitaker from Dr. Nigel G. F. Cooper's laboratory in the Department of Anatomical Sciences and Neurobiology at the University of Louisville. 


\subsubsection{Phosphodiesterases (PDEs)}

Phosphodiesterases (PDEs) are enzymes which act as regulators for signal transduction. Currently, there are 11 known families of PDEs. Each family includes three to four genes and each gene has three to eleven splice variants. PDEs degrade cyclic adenosine monophosphate (cAMP) and cyclic guanosine monophosphate (cGMP). Some PDEs, such as PDE4, PDE7 and PDE8, degrade cAMP only. Some of them degrade cGMP only, including PDE5, PDE6, and PDE9. The rest of the PDEs - PDE1, PDE2, PDE3, PDE10, and PDE11 - degrade both cAMP and cGMP (Figure 4.4).

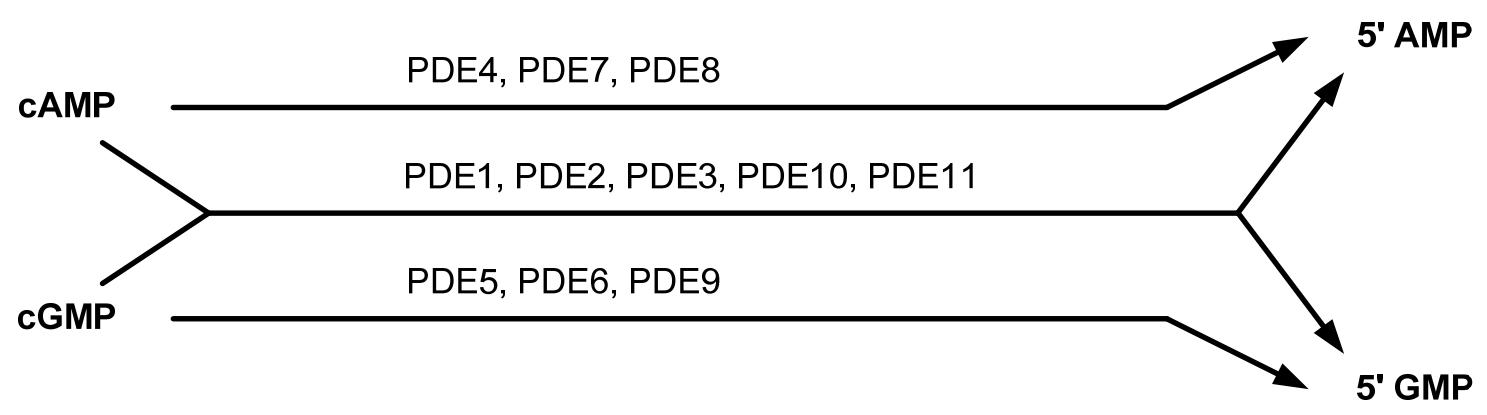

Figure 4.4: General scheme of cyclic 3', 5' nucleotide metabolism.

Image modified from Figure 1 in (157). Abbreviations are: cAMP, cyclic adenosine monophosphate; cGMP, cyclic guanosine monophosphate; PDE1-PDE11, types (gene families) of PDE isozymes. The lines reflect the catalytic capacity of the pathway.

In general, all the PDE isoforms contain a catalytic site. PDEs are differentiated based on the N-terminus regions. The PDE4 family represents the largest PDE family, constituted by four genes (PDE4A, PDE4B, PDE4C and PDE4D). Moreover, PDE4 expresses in all tissues. 


\subsubsection{Motivation}

Phosphodiesterase 4 (PDE4) is an enzyme that has been targeted for inflammatory disorders, including asthma and chronic obstructive pulmonary disease (COPD), as well as central nervous system (CNS) injury/disease. PDE4 has a large number of potential isoforms with apparent tissue and functional specificity. Potential roles of the different PDE4 isoforms within the CNS and other tissue types were studied using four known rat PDE4 paralogs (PDE4A, PDE4B, PDE4C and PDE4D) and their known isoforms. A computational method was designed to align rat ESTs to the corresponding chromosomal and mRNA sequences. The splice pattern and tissue type for each EST was examined in order to gain more insight into PDE4 isoform tissue specificity.

\subsubsection{Phosphodiesterase 4 (PDE4)}

PDE4, the most abundant PDE within the CNS (158), hydrolyzes cAMP (159) and selective inhibition by the drug rolipram (160). Recent studies have implicated PDE4 in many injury/disease models mostly through its role in inflammation (161) and have linked the enzyme to asthma, COPD and spinal code injury (162-164), making it important for study. Some researchers have even shown that PDE4D is found in the blood of African American females who have had strokes (165).

In the human genome, the PDE4 family contains four known genes (PDE4A-D). Each gene has about three to eleven splice variants, or isoforms. Each isoform is defined by its unique $\mathrm{N}$-terminus region. All have two to three conserved regions depending on the isoforms. These splice variants can be grouped into either long or short isoforms. 
Currently, there are four PDE4 isoform classes in the human genome. They are long, short, super-short and dead-short isoforms (See Figure 4.5). Long isoforms have upstream conserved region 1 (UCR1) and 2 (UCR2) domains. Short isoforms do not contain UCR1. Super-short isoforms have an N-terminally truncated UCR2. The deadshort isoforms lack UCR1 and UCR2 and have an inactive catalytic unit that is both Nand C- terminally truncated.
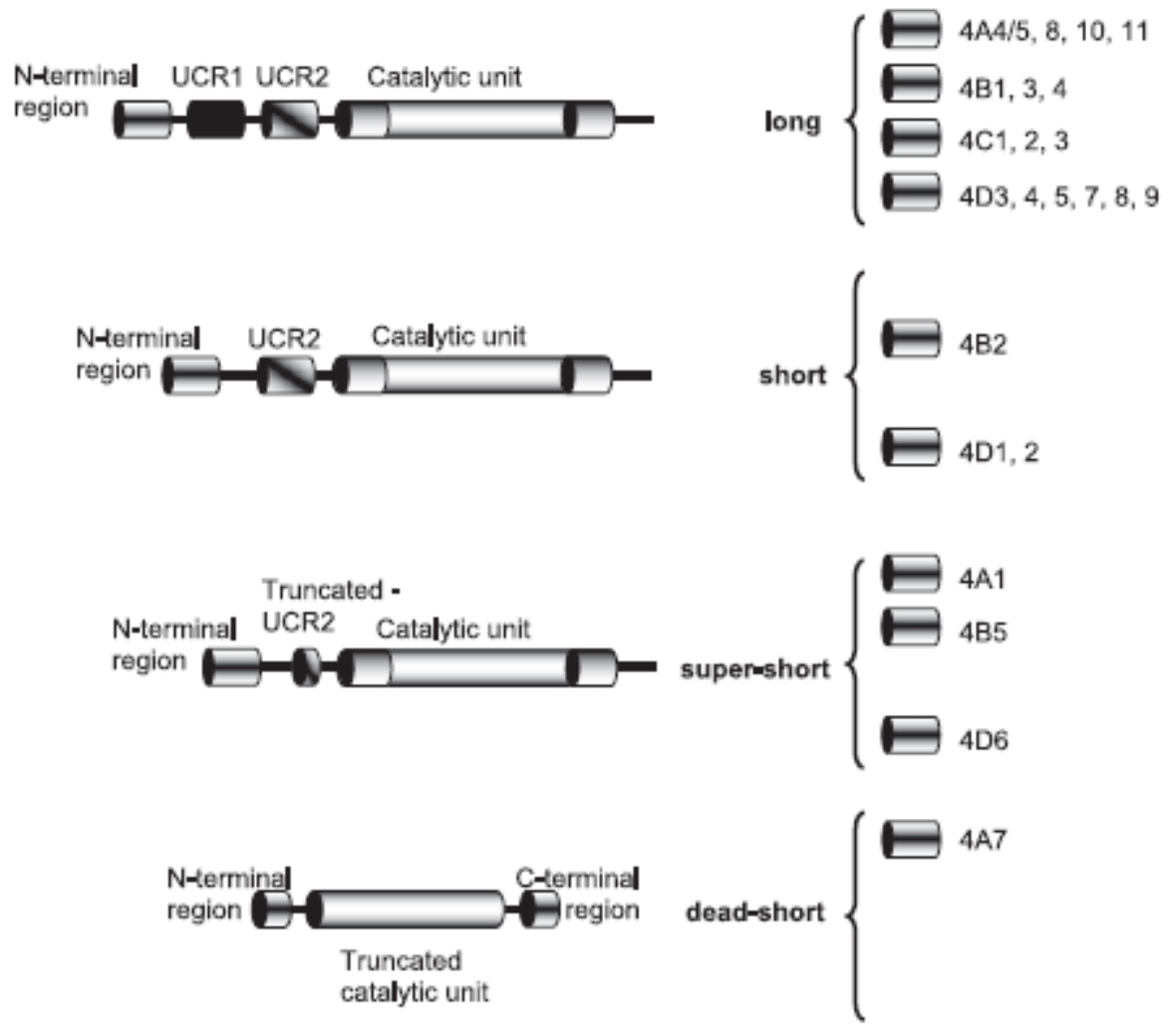

Figure 4.5: PDE4 isoform diversity: long, short, super-short and dead-short. Image adopted from Figure 2 in (166) and used by permission of author. 


\subsection{Methods for Computational Detection}

A project for detecting PDE4 splice variants in the rat genome was designed and implemented in silico in order to discover how PDE4 is spliced and determine tissue specificity of the splice variants

\subsubsection{Genomic Data}

Curated mRNA sequences for the rat PDE4A, PDE4B, PDE4C and PDE4D genes and known isoforms were downloaded from NCBI/RefSeq (73-76). Release 18, dated 717-2006 (http://www.ncbi.nlm.nih.gov/RefSeq/). The size of the sequences ranged from 1,000 to 3,000 base pairs. GenBank ids for each of these sequences are provided in Table 4.1. Each of the mRNAs were positioned within the UCSC rn4 goldenpath assembly of the rat genome (81) using WU blastn (70;167) and BLAT (168).

PDE4A localized to chromosome 8; PDE4B to chromosome 5; PDE4C to chromosome 16; and PDE4D to chromosome 2 (Table 4.1). The corresponding repeatmasked genomic regions were downloaded from the UCSC rn4 goldenpath assembly (7781) (http://genome.ucsc.edu/), dated 11-2004 including 30,000 bases flanking the 5' region of the first hit, and 30,000 bases flanking the 3' end of the final hit. 
Table 4.1: Rat PDE4 Family mRNA Sequence Information

\begin{tabular}{|c|c|c|c|c|c|c|}
\hline Gene & Variant & $\begin{array}{c}\text { GenBank } \\
\text { Accession }\end{array}$ & $\begin{array}{l}\text { GenBank } \\
\text { Identifier }\end{array}$ & $\begin{array}{l}\text { Gene } \\
\text { Length } \\
\text { (bp) }\end{array}$ & Chromosome & $\begin{array}{c}\text { Chromosomal } \\
\text { Length (bp) }\end{array}$ \\
\hline \multirow[t]{10}{*}{ PDE4A } & RefSeq & NM_013101.3 & 9681345 & 2,914 & chr8 & 68,640 \\
\hline & 1 & L36467.1 & 703258 & 2,778 & & \\
\hline & 2 & L27057.1 & 3334904 & 2,914 & & \\
\hline & 3 & AF110461.2 & 12057232 & 2,315 & & \\
\hline & 4 & L27062.1 & 436013 & 2,433 & & \\
\hline & 5 & M26715.1 & 203982 & 2,202 & & \\
\hline & 6 & M26716.1 & 203984 & 2,106 & & \\
\hline & 7 & M26717.1 & 203986 & 2,071 & & \\
\hline & 8 & M25348.1 & 206131 & 1,077 & & \\
\hline & 9 & M28411.1 & 206053 & 1,077 & & \\
\hline \multirow[t]{9}{*}{ PDE4B } & RefSeq & NM_017031.2 & 19923679 & 3,133 & chr5 & 573,258 \\
\hline & 1 & AF202732.1 & 16930142 & 3,022 & & \\
\hline & 2 & U95748.1 & 2352051 & 3,133 & & \\
\hline & 3 & ВС085704.1 & 55716044 & 2,511 & & \\
\hline & 4 & AF202733.1 & 16930144 & 2,433 & & \\
\hline & 5 & J04563.1 & 203967 & 2,158 & & \\
\hline & 6 & L27058.1 & 950096 & 2,647 & & \\
\hline & 7 & M25350.1 & 206126 & 1,080 & & \\
\hline & 8 & M28413.1 & 206055 & 1,080 & & \\
\hline \multirow[t]{3}{*}{ PDE4C } & & XM_214325.4 & 19220990 & 2,140 & chr16 & 23,713 \\
\hline & 1 & L27061.1 & 436011 & 1,764 & & \\
\hline & 2 & M25347.1 & 206129 & 1,077 & & \\
\hline \multirow[t]{12}{*}{ PDE4D } & RefSeq & NM_017032.1 & 8393931 & 2,077 & chr2 & $* 1,118,153$ \\
\hline & 1 & AF536979.1 & 22901889 & 2,348 & & \\
\hline & 2 & AF031373.1 & 2625099 & 2,470 & & \\
\hline & 3 & EF102484.1 & 118500411 & 2,285 & & \\
\hline & 4 & U09457.1 & 2564961 & 2,077 & & \\
\hline & 5 & AY388961.1 & 37256003 & 2,157 & & \\
\hline & 6 & EF121818.1 & 118772243 & 2,122 & & \\
\hline & 7 & L27059.1 & 436007 & 2,142 & & \\
\hline & 8 & L27060.1 & 3334454 & 2,834 & & \\
\hline & 9 & U09455.1 & 517501 & 1,963 & & \\
\hline & 10 & U09456.1 & 517416 & 1,821 & & \\
\hline & 11 & AF536974.1 & 22901879 & 1,756 & & \\
\hline
\end{tabular}

Abbreviations: bp, base pair; chr, chromosome.

*Mapping of the rat PDE4D gene indicates sequence assembly errors within chromosome 2. The total region covered includes 1,114,915 bp of chromosome 2, $154 \mathrm{bp}$ of chromosome 18 random fragments, and 3,084 bp of chromosome 2 random fragments. 


\subsubsection{Splice Detection}

Expressed sequence tags (ESTs) were downloaded from GenBank/dbEST release 154.0, dated 6-15-2006 (http://www.ncbi.nlm.nih.gov/dbEST/) for the purpose of understanding how the sequences were spliced for each of the four PDE4 genes. PDE4

mRNA sequences were downloaded from NCBI/RefSeq release 17, dated 5-5-2006 (http://www.ncbi.nlm.nih.gov/RefSeq/). Each EST passed through five steps in the detection of splice patterns: data preprocessing, sequence comparison, fine tuning, data analysis and pattern recognition. Figure 4.6 illustrates this process.

\section{Step 1: Data Preprocessing}

EST sequences were parsed to include only those from the species Rattus norvegicus. Raw EST and PDE4 mRNA sequences were masked for repetitive elements using RepeatMasker $(87 ; 88)$. Low complexity regions for the resulting rat ESTs and mRNAs were then masked using the DUST (70), SEG (70;90) and XNU (70) filters available from the wuBLAST software $(70 ; 167)$.

\section{Step 2: Sequence Comparison}

The four PDE4 mRNA sequences were aligned to their corresponding genomic regions using sim4 (107), which determines the most likely intron/exon boundaries of the mRNAs by taking into account known donor/acceptor sites. An initial alignment of the ESTs to the genomic regions was performed using WU blastn. 


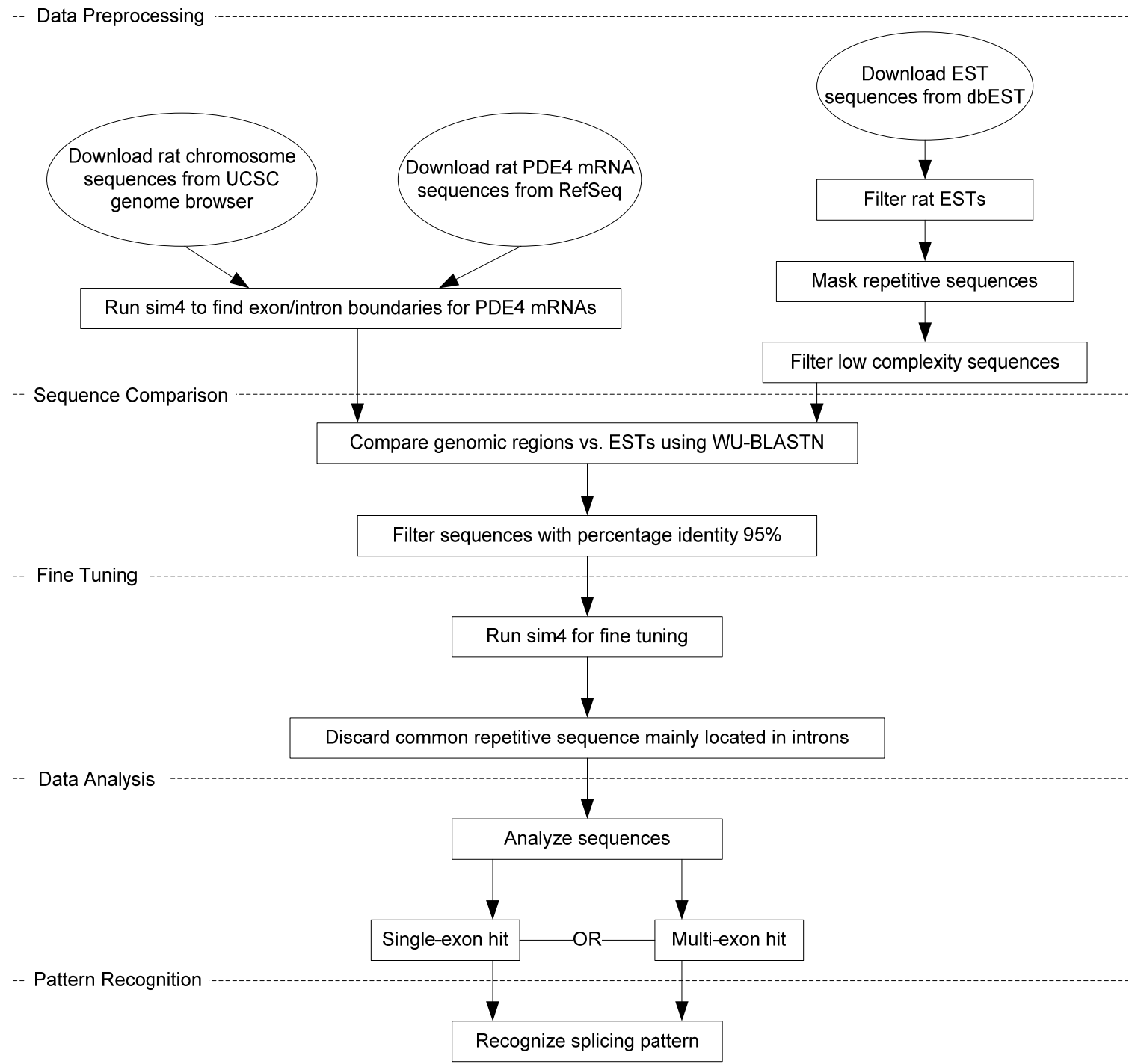

Figure 4.6: Process flow chart in computational detecting section. 


\section{Step 3: Fine Tuning}

EST sequences with less than $95 \%$ of percentage identity were filtered out, since these were not likely to be their true genomic locations. Remaining ESTs were realigned to the genome using sim4 (107), which returns the position where each EST appears in a chromosomal segment in accordance with known intron/exon boundary properties. The sim4 results were parsed to determine if the ESTs matched one contiguous region or if they spanned multiple regions, or exons. Each hit was therefore categorized as a singleexon hit or a multi-exon hit. Single-exon hits with a length less than $100 \mathrm{bp}$ were further filtered based on the number of hits in the region. If more than five ESTs hit the same region, all of ESTs for that region were discarded, since they most likely resulted from a previously uncharacterized repetitive element.

\section{Step 4: Data Analysis}

The ESTs remaining from Step 3 were divided into single-exon and multi-exon hit groups according to the gene to which they correspond: PDE4A, PDE4B, PDE4C or PDE4D. Each EST accession was searched against dbEST and UniGene (169). to determine the tissue type from which the EST was extracted, if known.

\section{Step 5: Pattern Recognition}

EST sequences that mapped to the same genomic location were placed in similar clusters. Overlapping patterns were examined for the maximum coverage length for each intron and exon. Each individual EST was examined for the pattern of exon and/or intron inclusion for the purpose of determining alternative splicing events, as well as possibly 
determining previously undetected exonic regions. Single-exon hits were further condensed, since it was unknown if these were spurious, or actual hits to exonic regions.

\subsection{Results in Exon Predictions}

Predicted exon locations for each of the four known PDE4 classes were determined based on the sim4 (107) and BLAT (168) alignments of the RefSeq and alternatively spliced mRNA sequences to the genomic regions. PDE4A splits into thirtythree regions (exons) on chromosome 8 (Table 4.2), with a noticeable gap between patterns twelve and eighteen. Examination of the genomic assembly for this region indicates an unsequenced gap that should be closed with future sequencing and assembly. Regions of the RefSeq and nine known isoforms corresponding to this gapped region were multiply aligned using clustalW (170) to produce five aligned regions where a gap exists in the genome assembly. PDE4B maps to chromosome 5, and is split into 15 exons (Table 4.3) while PDE4C divided into 16 exons on chromosome 16 (Table 4.4). The results for the PDE4D class are more puzzling. One large 897 bp gap in the mRNA sequence exists after the predicted location for exon 11 . There are $16.7 \mathrm{Mb}$ that separates the predicted terminal exon from the rest of the structure (Table 4.5). In addition, the most likely hits for this 897 bp region are to unassembled fragments assigned to chromosome 18 of the rat genome. The most likely explanation for this missing region is incorrect assembly of the rat genome. 
Table 4.2: PDE4A Exon Locations

\begin{tabular}{|c|c|c|c|c|c|c|c|c|c|c|c|c|c|c|}
\hline & $\begin{array}{l}\text { Region } \\
\text { Num. }\end{array}$ & CHROMOSOME 8 & $\begin{array}{c}\text { NM_013101.3 } \\
\text { (2914 BP) }\end{array}$ & $\begin{array}{l}\text { L36467.1 } \\
\text { (2778 BP) }\end{array}$ & $\begin{array}{l}\text { L27057.1 } \\
\text { (2914 BP) }\end{array}$ & $\begin{array}{l}\text { AF110461.2 } \\
\text { (2315 BP) }\end{array}$ & $\begin{array}{l}\text { L27062.1 } \\
\text { (2433 BP) }\end{array}$ & $\begin{array}{l}\text { M26715.1 } \\
\text { (2202 BP) }\end{array}$ & $\begin{array}{l}\text { M26716.1 } \\
\text { (2106 BP) }\end{array}$ & $\begin{array}{l}\text { M26717.1 } \\
\text { (2071 BP) }\end{array}$ & $\begin{array}{l}\text { M25348.1 } \\
\text { (1077 BP) }\end{array}$ & $\begin{array}{l}\text { M28411.1 } \\
\text { (1077 BP) }\end{array}$ & $\begin{array}{c}14-1 \\
\text { Testes (F) }\end{array}$ & $\begin{array}{c}14-1 \\
\text { Testes (R) }\end{array}$ \\
\hline & 1 & $\begin{array}{c}\text { CHR1 } \\
206,689,345 \\
206,689,357\end{array}$ & & & & & & & & 1-13 & & & & \\
\hline & 2 & $\begin{array}{c}\text { CHR1 } \\
206,689,447 \\
206,689,554\end{array}$ & & & & & & & & 14-121 & & & & \\
\hline & 3 & $20,176,941-20,176,964$ & & $7-30$ & & & & & & & & & & \\
\hline & 4 & $20,185,770-20,185,837$ & & $31-98$ & & & & & & & & & & \\
\hline & 5 & $20,189,322-20,189,507$ & & $99-284$ & & & & & & & & & & \\
\hline & 6 & $20,192,677-20,193,048$ & 23-394 & & 23-394 & & & & & & & & & \\
\hline & 7 & $20,210,557-20,210,642$ & & & & $1-86$ & & & & & & & & \\
\hline & 8 & $20,216,974-20,217,165$ & $395-586$ & $285-476$ & $395-586$ & $87-278$ & & & & & & & & \\
\hline & 9 & $20,218,045-20,218,081$ & $587-623$ & $477-513$ & $587-623$ & $279-315$ & & & & & & & & \\
\hline & 10 & $20,218,173-20,218,243$ & $624-694$ & 514-584 & 624-694 & $316-386$ & & & & & & & & \\
\hline & 11 & $20,221,145-20,221,194$ & $695-744$ & $585-634$ & $695-744$ & $387-436$ & & & & & & & & \\
\hline & 12 & $20,221,284-20,221,396$ & $745-857$ & $635-747$ & $745-857$ & $437-549$ & & & & & & & & \\
\hline & 13 & $x x, y x x x, x x x-x x, y x x, y x x$ & & & & & $1-266$ & & & & & & & \\
\hline & 14 & $x x, x x x, x x x-x x, x x x, y x x$ & & & & & $267-387$ & $1-121$ & & & & & & \\
\hline & 15 & $x x, x x x, x x x-x x, x x x, y x x$ & $858-901$ & $748-791$ & $858-901$ & $550-593$ & $388-431$ & $122-165$ & $26-69$ & & & & & \\
\hline & 16 & $x x, y x x, y x x-x x, y x x, y x x$ & $902-930$ & $792-820$ & $902-930$ & $594-622$ & $432-460$ & $166-194$ & $70-98$ & $134-162$ & & & & \\
\hline$a$ & 17 & $\mathrm{xx}, \mathrm{xxx}, \mathrm{xxx}-\mathrm{xx}, \mathrm{xxx}, \mathrm{xxx}$ & & & & & $461-480$ & $195-214$ & $99-118$ & 163-182 & & & & \\
\hline & 18 & $20,227,838-20,227,857$ & $931-950$ & $821-840$ & $931-950$ & $623-642$ & & & & & & & & \\
\hline & & $20,230,313-20,230,392$ & & & & & & & & & & & 3-83 & \\
\hline & 19 & $20,230,483-20,230,572$ & $951-1040$ & $841-930$ & $951-1040$ & $643-732$ & $481-570$ & $215-304$ & $119-208$ & $183-272$ & & & $\begin{array}{ll}84-172 \\
\end{array}$ & $150-240$ \\
\hline & 20 & $20,230,573-20,230,680$ & 1041-1148 & 931-1038 & 1041-1148 & $733-840$ & $571-678$ & $305-412$ & $209-316$ & $273-380$ & 1-108 & 1-108 & $173-280$ & $241-348$ \\
\hline & 21 & $20,232,551-20,232,649$ & 1149-1247 & 1039-1137 & 1149-1247 & $841-939$ & $679-777$ & $413-511$ & $317-415$ & & $109-207$ & $109-207$ & $281-379$ & $349-450$ \\
\hline & 22 & $20,232,738-20,232,902$ & $1248-1412$ & 1138-1302 & $1248-1412$ & $940-1104$ & $778-942$ & $512-676$ & $416-580$ & $381-545$ & $208-372$ & $208-372$ & $380-544$ & $451-615$ \\
\hline & 23 & $20,234,075-20,234,124$ & 1413-1512 & 1303-1352 & 1413-1512 & 1105-1154 & & $677-726$ & $581-630$ & $546-595$ & $373-422$ & $373-422$ & $545-595$ & $616-665$ \\
\hline & 24 & $20,234,125-20,234,174$ & & 1353-1402 & & 1155-1204 & 943-1042 & $727-776$ & $631-680$ & $596-645$ & $423-472$ & $423-472$ & $596-645$ & $666-715$ \\
\hline & 25 & $20,234,682-20,234,836$ & 1513-1667 & 1402-1557 & 1513-1667 & 1205-1359 & 1043-1197 & $777-931$ & $681-835$ & $646-800$ & $473-627$ & $473-627$ & $\begin{array}{l}646-711 \ldots \\
20234745\end{array}$ & \begin{tabular}{|c|c|}
$716-769 \ldots$ \\
$20,234,735$ \\
\end{tabular} \\
\hline & 26 & $20,235,014-20,235,136$ & $1668-1790$ & $1558-1680$ & $1668-1790$ & $1360-1482$ & $1198-1320$ & $932-1054$ & $836-958$ & $801-923$ & $628-750$ & $628-750$ & & \\
\hline & 27 & $20,236,456-20,236,638$ & 1791-1973 & 1681-1863 & 1791-1973 & 1483-1665 & 1321-1503 & 1055-1237 & $959-1141$ & $924-1106$ & $751-933$ & $751-933$ & & \\
\hline & 28 & $20,240,619-20,240,762$ & 1974-2117 & 1864-2007 & 1974-2117 & 1666-1809 & 1504-1647 & 1238-1381 & $1142-1285$ & 1107-1250 & 934-1077 & $934-1077$ & & \\
\hline & 29 & $20,240,763-20,241,268$ & $2118-2623$ & 2008-2513 & $2118-2623$ & 1810-2315 & $1648-2153$ & 1382-1887 & 1286-1791 & 1251-1756 & & & & \\
\hline & 30 & $20,241,269-20,241,527$ & 2624-2882 & $2514-2772$ & $2624-2882$ & & $2154-2411$ & 1888-2148 & 1792-2052 & 1757-2017 & & & & \\
\hline & 31 & $20,241,528-20,241,541$ & 2883-2896 & & 2883-2896 & & $2412-2425$ & $2149-2162$ & 2053-2066 & 2018-2031 & & & & \\
\hline & 32 & $20,241,542-20,241,557$ & 2897-2912 & & 2897-2912 & & & $2163-2178$ & 2067-2082 & 2032-2047 & & & & \\
\hline & 33 & $20,241,558-20,241,581$ & & & & & & $2179-2202$ & 2083-2106 & 2048-2071 & & & & \\
\hline
\end{tabular}

Regions 8, 9, 10 (in red) correspond to the UCR1 region. Regions 12-17 (blue) correspond to the UCR2 region. Regions 20-29 (green) correspond to the catalytic region. The last two columns indicate the mapped sequence for 14-1 from testis tissue. Abbreviations: $F$, forward strand; R: reverse strand. 
Table 4.3: PDE4B Exon Locations

\begin{tabular}{|c|c|c|c|c|c|c|c|c|c|c|c|c|c|c|c|c|c|c|}
\hline Reg & CHR 5 & $\begin{array}{c}\text { NM } 017031 \\
3133 \text { BP }\end{array}$ & $\begin{array}{c}\text { AFF202732 } \\
3022 \text { BP }\end{array}$ & \begin{tabular}{|l|} 
U95748 \\
3133 BP
\end{tabular} & $\begin{array}{c}\text { BC085704 } \\
2511 \text { BP }\end{array}$ & $\begin{array}{l}\text { AFF202733 } \\
20433 \mathrm{BP}\end{array}$ & $\begin{array}{l}J 04563 \\
2158 \mathrm{BP}\end{array}$ & $\begin{array}{l}277058 \\
2647 \mathrm{BP}\end{array}$ & $\begin{array}{l}\text { M223550 } \\
1080 \mathrm{BP}\end{array}$ & $\begin{array}{l}\text { M28413 } \\
1080 \mathrm{BP}\end{array}$ & $\begin{array}{l}15.1 \mathrm{~F} \\
\text { Cerebe. }\end{array}$ & 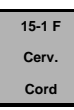 & $\begin{array}{c}23 \cdot 1 \\
\text { F } \\
\text { Brain }\end{array}$ & $\begin{array}{c}23 \cdot 1 \\
\text { R } \\
\text { Brain }\end{array}$ & 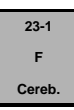 & $\begin{array}{c}23 \cdot 1 \\
\text { R } \\
\text { Cereb. }\end{array}$ & $\begin{array}{l}23.1 \mathrm{~F} \\
\text { Retina }\end{array}$ & $\begin{array}{c}\text { 23.1(R) } \\
\text { Retina }\end{array}$ \\
\hline 1 & $122,960,641-122,960,883$ & & $10-253$ & & & & & & & & & & & & & & & \\
\hline 2 & $123,067,107-123,067,219$ & & $254-3666$ & & & & & & & & & & & & & & & \\
\hline 3 & $123,079,695-123,079,933$ & & $367-605$ & & & & & & & & & & & & & & & \\
\hline 4 & $\begin{array}{l}123,158,156-123,158,005 \\
\end{array}$ & 16-465 & & 16-465 & & & & & & & & & & & & & & \\
\hline \multirow[t]{4}{*}{5} & $123,158,606-123,158,887$ & $466-747$ & & $466-747$ & $1-282$ & & & & & & & & & & & & & \\
\hline & $123,168,982-123,168,984$ & & & & & & & & & & & 1-3 & & & & & & \\
\hline & $123,168,985-123,169,154$ & & & & & & & & & & 1-172 & $4-174$ & & & & & & \\
\hline & $123,169,166-123,169,172$ & & & & & & & & & & 173-179 & 175-181 & & & & & & \\
\hline \multirow{5}{*}{$\frac{8}{9}$} & $123,351,697-123,351,999$ & & & & & $8: 311$ & & & & & & & & & & & & \\
\hline & & $748-942$ & $600-800$ & $\begin{array}{lll}748-942 \\
\end{array}$ & $283-477$ & $312-506$ & & & & & & & & & & & & \\
\hline & $123,426,399-123,426,355$ & $943-979$ & $801-837$ & $943-979$ & 478.514 & $507-543$ & & & & & & & & & & & & \\
\hline & $\begin{array}{l}123,426,452-123,426,460 \\
\end{array}$ & $980-988$ & $838-846$ & 980.988 & 515-523 & 544.552 & & & & & & & & & & & & \\
\hline & 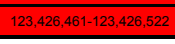 & 989-1050 & $\begin{array}{ll}84-908 \\
\end{array}$ & $989-1050$ & 524-585 & 553-614 & $\begin{array}{l}1-62 \\
\end{array}$ & & & & & & & & & & & \\
\hline 11 & 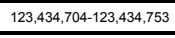 & $1051-1100$ & $\begin{array}{l}909-958 \\
\end{array}$ & 1051-1100 & $586-635$ & $\begin{array}{l}615-664 \\
\end{array}$ & $63-112$ & & & & & & & & & & & \\
\hline 12 & 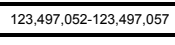 & & & & & & & $1-6$ & & & & & & & & & & \\
\hline 13 & $123,497,150-123,497,802$ & & & & & & & 7.659 & & & & & & & & & & \\
\hline \multirow{6}{*}{$\begin{array}{l}14 \\
15\end{array}$} & $123,497,803-123,497,915$ & 1101-12213 & 959-1071 & $1101-1213$ & $\begin{array}{l}636-748 \\
\end{array}$ & $6655-777$ & 113-225 & $660-772$ & & & & & & & & & & \\
\hline & $123,517,627-123,517,629$ & & & & & & & & & & & & & & & & & $2-4$ \\
\hline & $\begin{array}{l}123,517,630-123,517,648 \\
123,517,649-123,517,656\end{array}$ & & & & 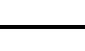 & & 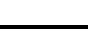 & & & & & & 8.15 & $\begin{array}{l}1-19 \\
20-27\end{array}$ & 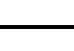 & $\frac{1-19}{20-27}$ & - & $\begin{array}{l}5.23 \\
24.31\end{array}$ \\
\hline & 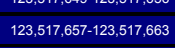 & & & & & & & & & & & & $16-22$ & 28.34 & 222.28 & & & 32.38 \\
\hline & $123,517,664-123,517,899$ & & & & & & & & & & & & 232.259 & 355270 & 29.264 & $35-272$ & $25-260$ & 39.275 \\
\hline & $122,518,627-123,518,720$ & 1214-1307 & 1072-1165 & 1214-1307 & $749 \cdot 842$ & $778-871$ & 226.319 & $773-866$ & & & & & $260-354$ & 277.365 & $265-358$ & $273-369$ & $261-354$ & $276-370$ \\
\hline 16 & $123,523,794-123,523,8661$ & $1300-1375$ & $1166-1233$ & $1300-1735$ & $843-910$ & $872-939$ & $\begin{array}{lll}320-387 & \\
\end{array}$ & $\begin{array}{ll}867-934 \\
\end{array}$ & & & & & $355-422$ & $366-433$ & $359 \cdot-426$ & $370-437$ & $355-422$ & $377-1438$ \\
\hline 18 & $123,523,862-123,523,972$ & 1376-14866 & 1234-1344 & 1376-1486 & 911-1021 & 940-1050 & 388-498 & 935-1045 & 1-111 & 1-111 & & & 423-497 & 4344486 & 427-496 & 438-487 & 423-497 & 439-489 \\
\hline 19 & 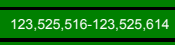 & 14877-1585 & $13455-1443$ & 14877-1585 & 1022-1120 & 1051-1149 & 499.597 & 1046-1144 & 112-210 & $112-210$ & & & & & & & & \\
\hline 20 & $123,525,751-123,525,915$ & $1586-1750$ & $1444-1608$ & $1586-1750$ & $1121-1285$ & $11150-1314$ & $598-762$ & 1145-1309 & $211-375$ & $211-375$ & & & & & & & & \\
\hline 21 & $123,527,679-123,527,778$ & 1751-1850 & $1609-1708$ & $1751-1850$ & 1286-1385 & $13151-1414$ & $\begin{array}{ll}763-862 \\
\end{array}$ & 1310-14099 & $376-475$ & $376-475$ & & & & & & & & \\
\hline 22 & $123,529,535-123,529,6899$ & 1851-2005 & $1709-1663$ & 1851-2005 & $1336-1540$ & $1415-1569$ & $863-1017$ & 1410-1564 & $476-630$ & $476-630$ & & & & & & & & \\
\hline 23 & $123,529,781-123,529,903$ & $\begin{array}{l}2006-2128 \\
\end{array}$ & $1864-1986$ & $2006-2128$ & 1541-1663 & $1570-1692$ & 1018-1140 & $\begin{array}{l}1565-16877 \\
\end{array}$ & $\begin{array}{l}631-753 \\
\end{array}$ & 631-753 & & & & & & & & \\
\hline 24 & $123,580,570-123,550,752$ & $2129 \cdot-2311$ & 1987-2169 & $2129 \cdot 2311$ & $1664-1846$ & $1693-1875$ & 1141-1323 & $\begin{array}{ll}1688-1870 \\
\end{array}$ & $754-936$ & $754 \cdot 936$ & & & & & & & & \\
\hline 25 & $123,533,054-123,533,197$ & $2312-2455$ & $2170-2313$ & $2312-2455$ & 1847-1990 & 1876-2019 & 1324-1467 & 1871-2014 & $937-1080$ & $\begin{array}{ll}937-1080 \\
\end{array}$ & & & & & & & & \\
\hline 26 & $123,533,198-123,533,335$ & $\begin{array}{ll}2456-2593 \\
2\end{array}$ & $2314-2451$ & $2456-25933$ & 1991-2128 & $2020-2157$ & $1468-1605$ & $2015-2152$ & & & & & & & & & & \\
\hline 27 & $123,53,3336-123,53,53,612$ & $2594-2870$ & $2452-2728$ & $2594-2870$ & & $2158-2433$ & $1006-1882$ & 2155-2429 & & & & & & & & & & \\
\hline 28 & $122,533,6131-123,533,832$ & $2871-3090$ & $2729-2948$ & $2871-13000$ & & & 1882-2101 & $2430-2647$ & & & & & & & & & & \\
\hline 29 & $123,533,833-123,533,877$ & 3091-3133 & $2949-2993$ & 3091-3133 & & & 210222146 & & & & & & & & & & & \\
\hline 30 & 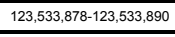 & & $2994-3006$ & & & & $2146-2158$ & & & & & & & & & & & \\
\hline 31 & $123,533,891-123,533,899$ & & $3007-3015$ & & & & & & & & & & & & & & & \\
\hline
\end{tabular}

Regions 7-10 (in red) correspond to the UCR1 region. Regions 14-16 (blue) correspond to the UCR2 region. Regions 18-26 (green) correspond to the catalytic region. Abbreviations: F, forward strand; R: reverse strand. 
Table 4.4: PDE4C Exon Locations

\begin{tabular}{|c|c|c|c|c|}
\hline Region & CHR 16 & $\begin{array}{c}\text { XM_214325.4 } \\
2140 \mathrm{BP}\end{array}$ & $\begin{array}{l}\text { L27061.1 } \\
1764 \text { BP }\end{array}$ & $\begin{array}{l}\text { M25347.1 } \\
1077 \mathrm{BP}\end{array}$ \\
\hline 1 & $19,220,990-19,220,909$ & 1-82 & & \\
\hline 2 & 19,209,993-19,209,849 & 83-227 & & \\
\hline 3 & $19,202,151-19,201,990$ & 228-389 & & \\
\hline 4 & $19,201,989-19,201,960$ & $390-419$ & 7-36 & \\
\hline 5 & $19,201,551-19,201,515$ & $420-456$ & $37-73$ & \\
\hline 6 & $19,201,430-19,201,360$ & $457-527$ & 74-144 & \\
\hline 7 & $19,201,236-19,201,184$ & $528-580$ & 145-197 & \\
\hline 8 & $19,200,897-19,200,785$ & $581-693$ & $198-310$ & \\
\hline 9 & $19,200,708-19,200,615$ & 694-787 & 311-404 & \\
\hline 10 & 19,200,243-19,200,191 & $788-840$ & $405-457$ & \\
\hline 11 & $19,200,190-19,200,083$ & 841-948 & $458-565$ & 1-108 \\
\hline 12 & $19,199,997-19,199,899$ & 949-1047 & $566-664$ & $109-207$ \\
\hline 13 & $19,199,679-19,199,515$ & 1048-1212 & $665-829$ & 208-372 \\
\hline 14 & $19,199,436-19,199,337$ & 1213-1312 & 830-929 & 373-472 \\
\hline 15 & $19,199,125-19,198,971$ & 1313-1467 & $930-1084$ & $473-627$ \\
\hline 16 & $19,198,573-19,198,451$ & $1468-1590$ & 1085-1207 & $628-750$ \\
\hline 17 & $19,198,259-19,198,077$ & 1591-1773 & $1208-1390$ & $751-933$ \\
\hline 18 & $19,197,643-19,197,500$ & 1774-1917 & 1391-1534 & 934-1077 \\
\hline 19 & $19,197,499-19,197,277$ & $1918-2140$ & 1535-1757 & \\
\hline
\end{tabular}

Regions 3-6 (in red) correspond to the UCR1 region. Regions 8-9 (blue) correspond to the UCR2 region. Regions 11-19 (green) correspond to the catalytic region. 
Table 4.5: PDE4D Exon Locations

\begin{tabular}{|c|c|c|c|c|c|c|c|c|c|c|c|c|c|}
\hline Region & CHR 2 & $\begin{array}{c}\text { NM_017032.1 } \\
2077 \text { BP }\end{array}$ & $\begin{array}{c}\text { AF536979.1 } \\
2348 \mathrm{BP}\end{array}$ & $\begin{array}{l}\text { AF031373.1 } \\
2470 \mathrm{BP}\end{array}$ & $\begin{array}{c}\text { EF102484.1 } \\
2285 \text { BP }\end{array}$ & $\begin{array}{l}\text { U09457.1 } \\
2077 \mathrm{BP}\end{array}$ & $\begin{array}{c}\text { AY388961 } \\
2157 \text { BP }\end{array}$ & $\begin{array}{c}\text { EF121818.1 } \\
2122 \mathrm{BP}\end{array}$ & $\begin{array}{l}\text { L27059.1 } \\
2142 \text { BP }\end{array}$ & $\begin{array}{l}\text { L27060.1 } \\
2834 \text { BP }\end{array}$ & $\begin{array}{l}\text { U09455.1 } \\
1963 \mathrm{BP}\end{array}$ & $\begin{array}{l}\text { U09456.1 } \\
1821 \mathrm{BP}\end{array}$ & $\begin{array}{c}\text { AF536974.1 } \\
1756 \mathrm{BP}\end{array}$ \\
\hline 1 & $40,196,097-40,196,221$ & & $1-125$ & & & & & & & & & & \\
\hline 2 & $40,394,274-40,394,503$ & & $126-355$ & & & & & & & & & & \\
\hline 3 & $40,496,293-40,496,732$ & & & $1-440$ & & & & & & & & & \\
\hline 4 & $40,644,734-40,645,025$ & & & & 1-292 & & & & & & & & \\
\hline 5 & $40,820,287-40,820,333$ & $1-47$ & & & & $1-47$ & & & & & & & \\
\hline 6 & $41,010,800-41,010,928$ & & & & & & & & & & & & \\
\hline 7 & $41,063,673-41,063,737$ & & & & & & $1-65$ & 1-129 & & & & & \\
\hline 8 & $41,114,936-41,115,127$ & $48-239$ & $356-547$ & $441-632$ & $293-484$ & $48-239$ & $66-257$ & $130-321$ & & & & & \\
\hline 9 & $41,131,201-41,131,237$ & $240-276$ & $548-584$ & $633-669$ & $485-521$ & $240-276$ & $258-294$ & $322-358$ & & & & & \\
\hline 10 & $41,136,439-41,136,449$ & $277-287$ & $585-595$ & $670-680$ & $522-532$ & $277-287$ & $295-305$ & $359-369$ & & & & & \\
\hline 11 & $41,136,450-41,136,512$ & $288-350$ & $596-658$ & $681-743$ & 533-595 & $288-350$ & $306-368$ & $370-432$ & $7-69$ & & & & \\
\hline 12 & $41,139,162-41,139,211$ & $351-400$ & $659-708$ & $744-793$ & $596-645$ & $351-400$ & $269-418$ & $433-482$ & $70-119$ & & & & \\
\hline 13 & $41,275,198-41,275,253$ & & & & & & & & & & $1-241$ & & \\
\hline 14 & $41,275,254-41,275,397$ & & & & & & & & & & $242-285$ & $1-144$ & \\
\hline 15 & $41,275,398-41,275,446$ & & & & & & & & & & $201-249$ & & \\
\hline 16 & $41,275,447-41,275,483$ & & & & & & & & & 13150 & $250-286$ & & \\
\hline 17 & $41,275,484-41,275,596$ & $401-513$ & $709-821$ & 794-906 & $646-758$ & $401-513$ & $419-531$ & $483-595$ & $120-232$ & $37-150$ & $287-399$ & $145-257$ & \\
\hline 18 & $41,299,092-41,299,320$ & & & & & & & & & & & & $1-229$ \\
\hline 19 & $41,305,886-41,305,979$ & 514-607 & $822-915$ & $907-1000$ & $759-852$ & 514-607 & $532-625$ & $596-689$ & $233-326$ & $151-244$ & $400-493$ & $258-351$ & $230-323$ \\
\hline 20 & $41,307,257-41,307,429$ & $608-780$ & $916-1088$ & 1001-1173 & $853-1025$ & $608-780$ & $626-798$ & $690-862$ & $327-499$ & $245-417$ & $\begin{array}{ll}494-666 \\
\end{array}$ & $352-524$ & $324-496$ \\
\hline 21 & $41,308,372-41,308,470$ & $781-879$ & 1089-1187 & 1174-1272 & 1026-1124 & $781-879$ & $799-897$ & $863-961$ & 500-598 & $418-516$ & $667-765$ & $525-623$ & $497-595$ \\
\hline 22 & $41,309,604-41,309,768$ & $880-1044$ & $1188-1352$ & $1273-1437$ & $1125-1289$ & $880-1044$ & $898-1062$ & $962-1126$ & $599-763$ & $517-681$ & $766-930$ & $624-788$ & $596-760$ \\
\hline \multirow[t]{2}{*}{23} & $41,310,913-41,311,012$ & $1045-1144$ & $1353-1452$ & $1438-1537$ & $1290-1389$ & $1045-1144$ & $1063-1162$ & $1127-1226$ & $764-863$ & $682-781$ & $931-1030$ & $789-888$ & $761-860$ \\
\hline & CHR 18 RANDOM & & & & & & & & & & & & \\
\hline \multirow[t]{2}{*}{24} & $266,263-266,417$ & 1145-1299 & $1453-1607$ & $1538-1692$ & $1390-1544$ & 1145-1299 & 1163-1317 & $1227-1381$ & $864-1018$ & $782-936$ & $1031-1185$ & $889-1043$ & $861-1015$ \\
\hline & CHR 2 RANDOM & & & & & & & & & & & & \\
\hline 25 & $1,582,343-1,582,465$ & $1300-1422$ & $1608-1730$ & $1693-1815$ & $1545-1667$ & $1300-1422$ & $1318-1440$ & $1382-1504$ & $1019-1141$ & $937-1059$ & $1186-1308$ & $1044-1166$ & $1016-1138$ \\
\hline 26 & $1,583,164-1,583,346$ & 1423-1605 & 1731-1913 & 1816-1998 & $1668-1850$ & $1423-1605$ & $1441-1623$ & $1505-1687$ & $1142-1324$ & $1060-1242$ & 1309-1491 & $1167-1349$ & $1139-1321$ \\
\hline 27 & $1,583,844-1,584,278$ & $1606-2040$ & 1914-2348 & $1999-2433$ & $1851-2285$ & $1606-2040$ & $1624-2058$ & $1688-2122$ & $1325-1759$ & 1243-1677 & 1492-1925 & $1350-1784$ & $1322-1756$ \\
\hline 28 & $1,584,279-1,584,308$ & $2041-2070$ & & $2434-2463$ & & $2041-2070$ & $2059-2088$ & & $1760-1789$ & $1678-1707$ & 1926-1955 & $1785-1814$ & \\
\hline 29 & $1,584,309-1,584,377$ & & & & & & $2089-2157$ & & $1790-1858$ & $1708-1776$ & & & \\
\hline 30 & $1,584,378-1,584,630$ & & & & & & & & 1859-2111 & $1777-2029$ & & & \\
\hline 31 & $\begin{array}{l}1,584,631-1,584,638 \\
\end{array}$ & $2071-2077$ & & $2464-2470$ & & 2071-2077 & & & $2112-2119$ & $2030-2037$ & 1956-1963 & $1815-1821$ & \\
\hline 32 & $1,584,639-1,584,644$ & & & & & & & & $2120-2126$ & 2038-2043 & & & \\
\hline 33 & $1,584,645-1,585,427$ & & & & & & & & & $2044-2827$ & & & \\
\hline
\end{tabular}




\subsubsection{Spliced Pattern Observation}

A total of 812,497 rat ESTs were extracted from dbEST and compared to the genomic regions for the PDE4 families (See Section 4.5). ESTs directly correlate to processed mRNA sequences expressed in the cell, and therefore yield information pertaining to the spliced exon patterns. While EST sequences contain information about adjacent exons alternatively spliced, they normally do not contain the whole isoform due to their short length (normally 200-500 bp). A breakdown of the tissue types in which ESTs were found for each of the four PDE4 families is given in Figure 4.7.
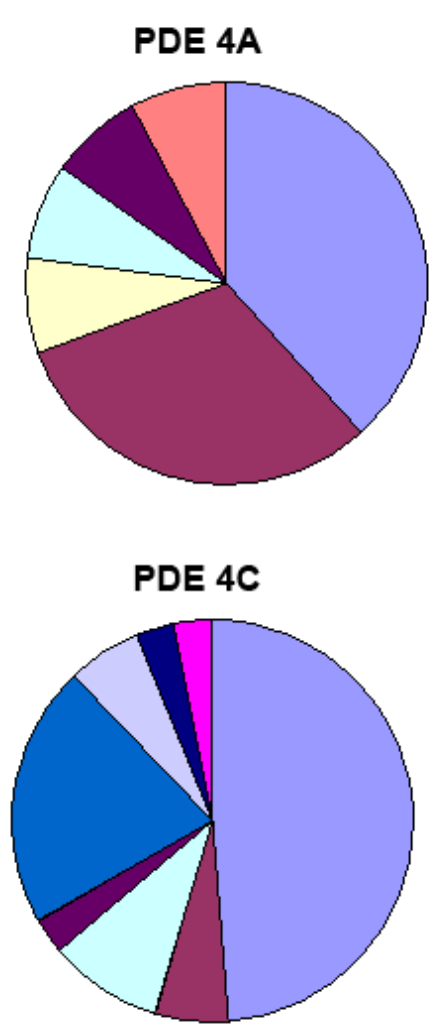
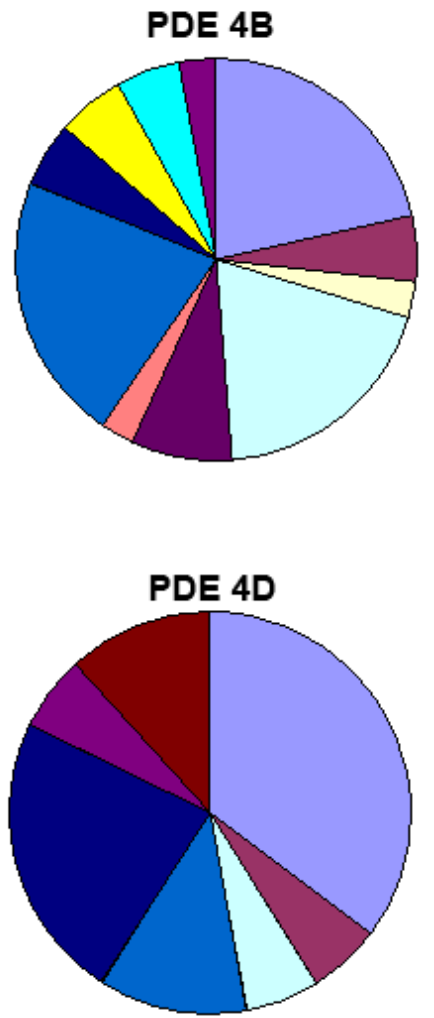

$\square$ Brain

$\square$ Genitourinary

$\square$ Kidney

$\square$ Pancreas

- Placenta

$\square$ Testis

$\square$ Heart

$\square$ Endocrine

口 Lung

$\square$ Vestibular Organs

$\square$ Liver

$\square$ Cervix

$\square$ Eye

- Cartilage

Figure 4.7: Tissue distribution of ESTs for each of the four PDE4 families. Unknown, mixed and uncharacterized tissue types have been removed. 
Variant tissue types were found in each PDE4 gene, when tracing the information from the GenBank data. Brain, genitourinary, heart and pancreas tissues were found in all PDE4 genes. Adipose and embryonic tissues were only associated with PDE4A; endocrine and vestibular end organs tissues were found specifically for PDE4B; the tissue types of cervix, dorsal root ganglia, hippocampus, hypothalamus and insulinoma were found with only PDE4C; and the cartilage tissue was only associated with PDE4D (Figure 4.7).

\subsubsection{PDE4A}

An initial filtering of EST hits with a percentage identity of $95 \%$ or greater yields 742 ESTs for the PDE4A region. After additional filtering and clustering, 27 ESTs were shown to map uniquely to the PDE4A region of chromosome 8. Eighteen of these were found to be unspliced ESTs, involving four different single-exon patterns. Another nine were spliced ESTs, representing nine separate multiple exon patterns (Tables 4.6 and 4.7). The ESTs for PDE4A were found in following tissues: four each for the brain and genitourinary system and one each for heart, adipose, embryonic, placenta, pineal gland, testis, pancreas, kidney and eye with the remainder coming from unknown, uncharacterized or mixed tissue.

Twelve of the single exon hits represented possible extensions of the $3^{\text {' }}$ untranslated region (UTR) of known PDE4A isoforms. Another EST hit, from accession BG372498.1 (pattern 1), indicated a potential new exon between regions six and seven from Table 4.2. This EST was derived from eye tissue, and therefore might have some tissue specificity. 
Table 4.6: Single Exon Splicing Patterns and Tissue Types for PDE4A

\begin{tabular}{cclllrr}
\hline Pattern & Accession & Tissue Type & Regions Covered & Notes & Chromosomal & locations \\
\hline 1 & BG372498.1 & eye & $(6-7)$ & new exon & $20,197,794$ & $20,198,264$ \\
2 & CB797062.1 & adipose & $28-29$ & & $20,240,617$ & $20,240,944$ \\
2 & CA506060.1 & embryonic & 29 & & $20,240,875$ & $20,241,552$ \\
2 & CR458483.1 & uncharacterized & $29-30-31$ & & $20,241,248$ & $20,241,539$ \\
2 & BF413211.1 & brain & $29-30-31-32$ & & $20,241,264$ & $20,241,552$ \\
3 & BG378996.1 & brain & $30-31-32-33$ & & $20,241,302$ & $20,241,574$ \\
3 & CV111386.1 & genitourinary & $30-3132-33-(33-)$ & 3' UTR extension & $20,241,477$ & $20,242,162$ \\
4 & AW507088.1 & brain & $(33-)$ & 3' UTR extension & $20,241,883$ & $20,242,178$ \\
4 & BF545214.1 & mixed & $(33-)$ & 3' UTR extension & $20,242,103$ & $20,242,242$ \\
4 & CR459446.1 & uncharacterized & $(33-)$ & 3' UTR extension & $20,242,379$ & $20,242,721$ \\
4 & Al029179.1 & mixed & $(33-)$ & 3' UTR extension & $20,242,531$ & $20,242,727$ \\
4 & AA999059.1 & unknown & $(33-)$ & 3' UTR extension & $20,242,535$ & $20,242,721$ \\
4 & BE120273.1 & mixed & $(33-)$ & 3' UTR extension & $20,242,546$ & $20,242,723$ \\
4 & BG381367.1 & heart & $(33-)$ & 3' UTR extension & $20,242,547$ & $20,242,727$ \\
4 & BQ192530.1 & uncharacterized & $(33-)$ & 3' UTR extension & $20,242,556$ & $20,243,158$ \\
4 & BF399702.1 & mixed & $(33-)$ & 3' UTR extension & $20,242,560$ & $20,242,721$ \\
4 & BE107614.1 & mixed & $(33-)$ & 3' UTR extension & $20,242,697$ & $20,243,128$ \\
4 & CR465548.1 & uncharacterized & $(33-)$ & 3' UTR extension & $20,242,792$ & $20,243,158$ \\
\hline
\end{tabular}

Five of the spliced EST patterns revealed potential new exons (Table 4.7). These occurred between regions two and three (pattern 10 from genitourinary tissue); between regions six and seven (pattern 13 from kidney tissue); between regions 18 and 19 (pattern 14 from genitourinary tissue); between regions 22 and 23 (pattern 8 from pancreas tissue); and between regions 24 and 25 (pattern 6a derived from whole brain tissue). The first three splice patterns (patterns 1, 10 and 13) occurred upstream of the UCR1 in the hypervariable N-terminal region. Pattern 14 produces a novel exon in the linker region between UCR2 and the conserved catalytic core while patterns 8 and 6a produced novel exons in the catalytical core of PDE4A. In addition, a new splicing pattern incorporating known exons was described by pattern 10, derived from genitourinary tissue. 
Table 4.7: Multiple Exon Splicing Patterns and Tissue Types for PDE4A

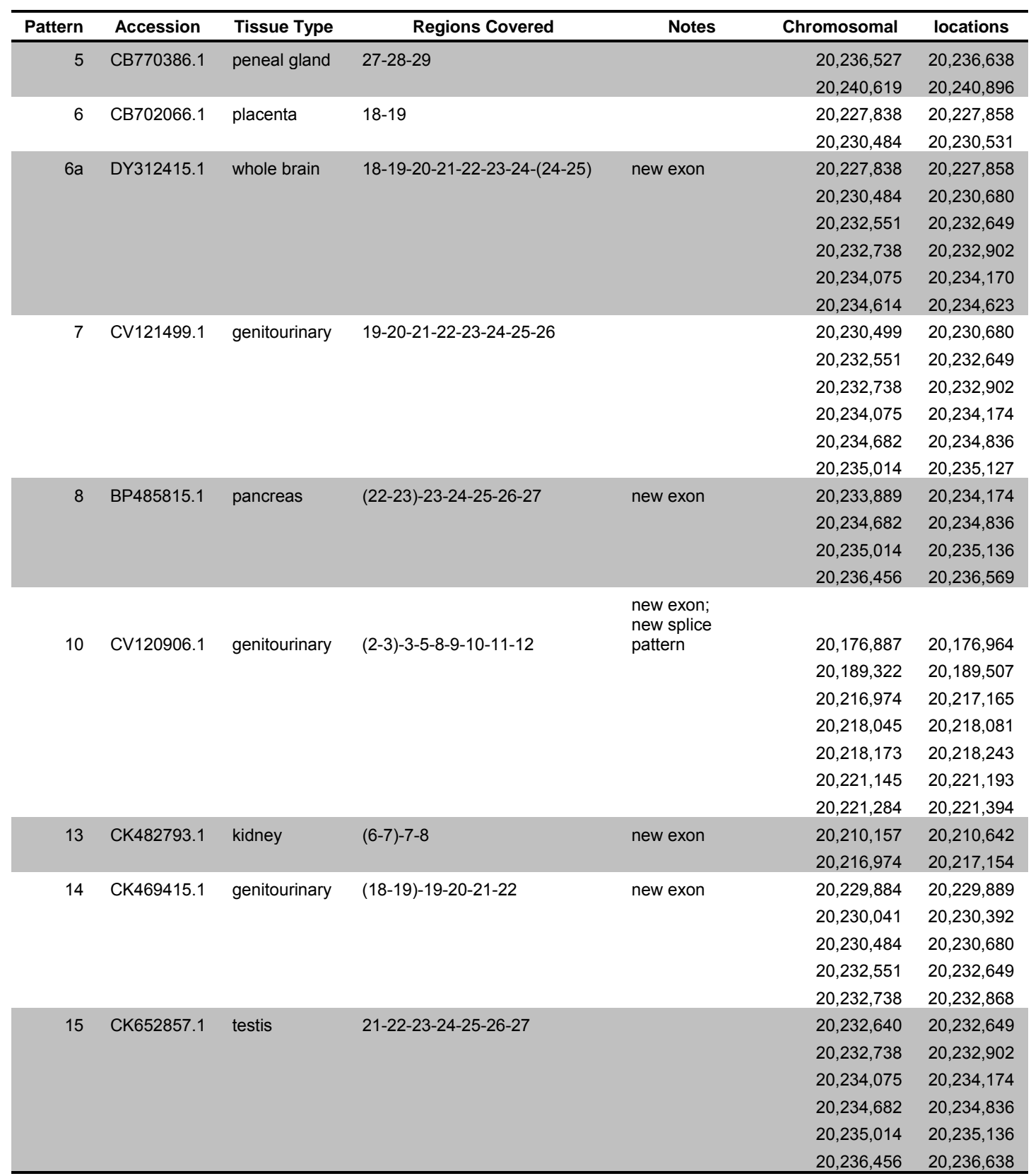




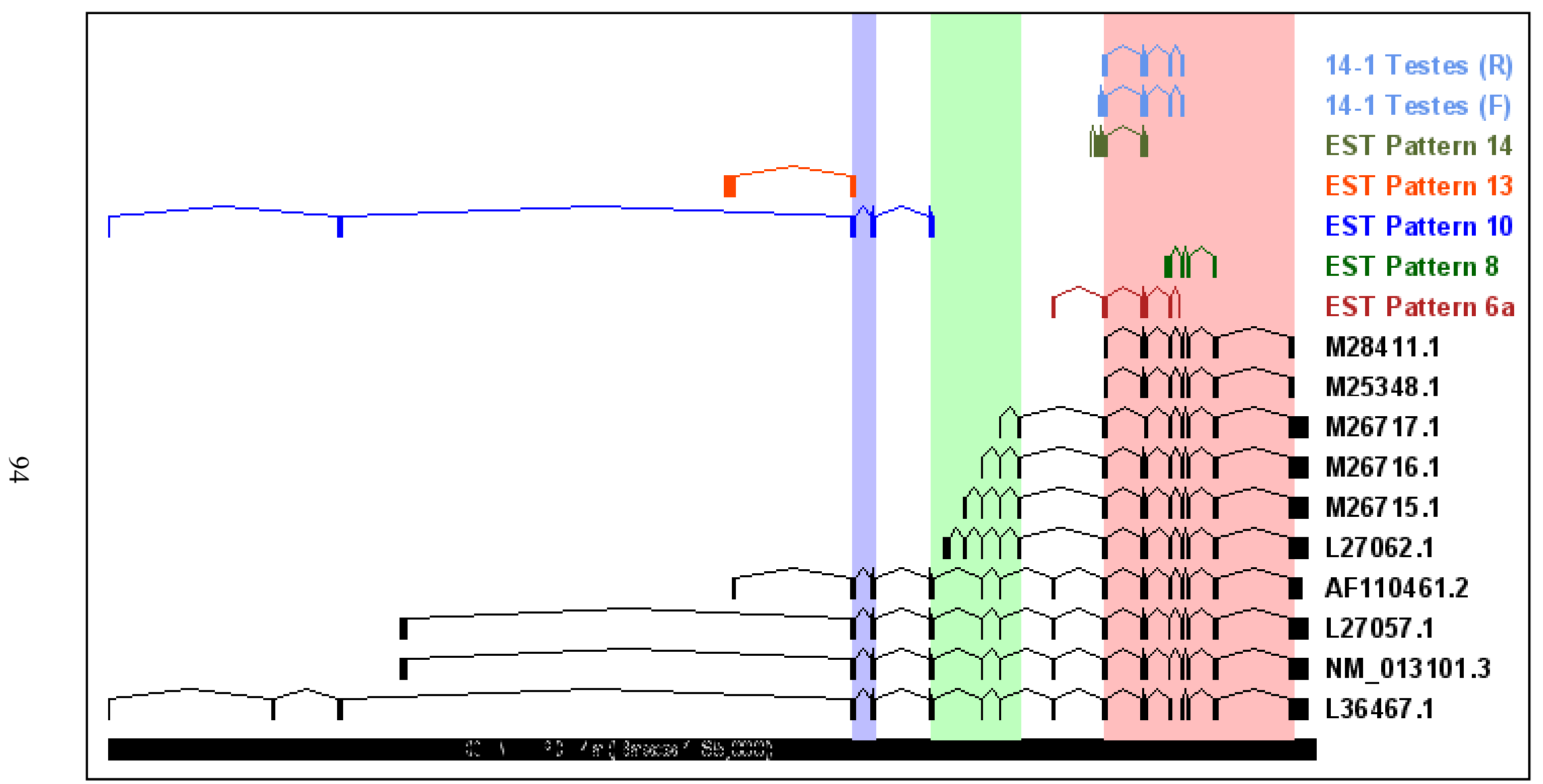

Figure 4.8: PDE4A isoforms, EST patterns and sequenced regions.

Abbreviations: $F$, forward strand; $R$, reverse strand. 


\subsubsection{PDE4B}

Initial filtering resulted in 5,112 ESTs which matched the PDE4B genomic region. After clustering and filtering again, 49 ESTs were found to map completely and uniquely to the PDE4B region of the rat chromosome 5. Thirty-eight of these ESTs were from single-exon hits covering 14 different regions of PDE4B while 11 were from multiple-exon hits representing seven different regions (Tables 4.8 and 4.9). Fifteen of the ESTs originated from brain, seven from heart, three from pancreas, two each from endocrine and genitourinary, and one each from vestibular end organs, lung, and placenta. The remaining were mixed, uncharacterized or unknown.

Eighteen of the single-exon hits indicated extensions of the 3' UTR region (pattern 10). Another 11 provide evidence for an exon between regions five and six (patterns 1, 2, 3, 4, 5, 6 and 17). Pattern 16 indicated a new exon between regions six and seven, while pattern 22 indicated a new exon between regions 11 and 12. In addition, two supported a previously discovered exon between regions 14 and 15 (patterns 7 and 8), and another indicated a novel exon between regions 20 and 21 (pattern 9).

Three of the spliced EST patterns indicated previously discovered exons. These include two exons between regions five and six (pattern 15), one exon between regions 11 and 12 (pattern 24), one exon between regions 14 and 15 (pattern 23), two exons between regions 15 and 16 (pattern 23), and one novel exon between regions 16 and 17 (pattern 23).

Of the novel predicted exons, a number were in the N-terminal domain (patterns $1,2,3,4,5,6,15,16$ and 17) while two were in the linker region between UCR1 and UCR2 (patterns 22 and 24), five were in the UCR2 region (patterns 7, 8 and three from 
Table 4.8: Single Exon Splicing Patterns and Tissue Types for PDE4B

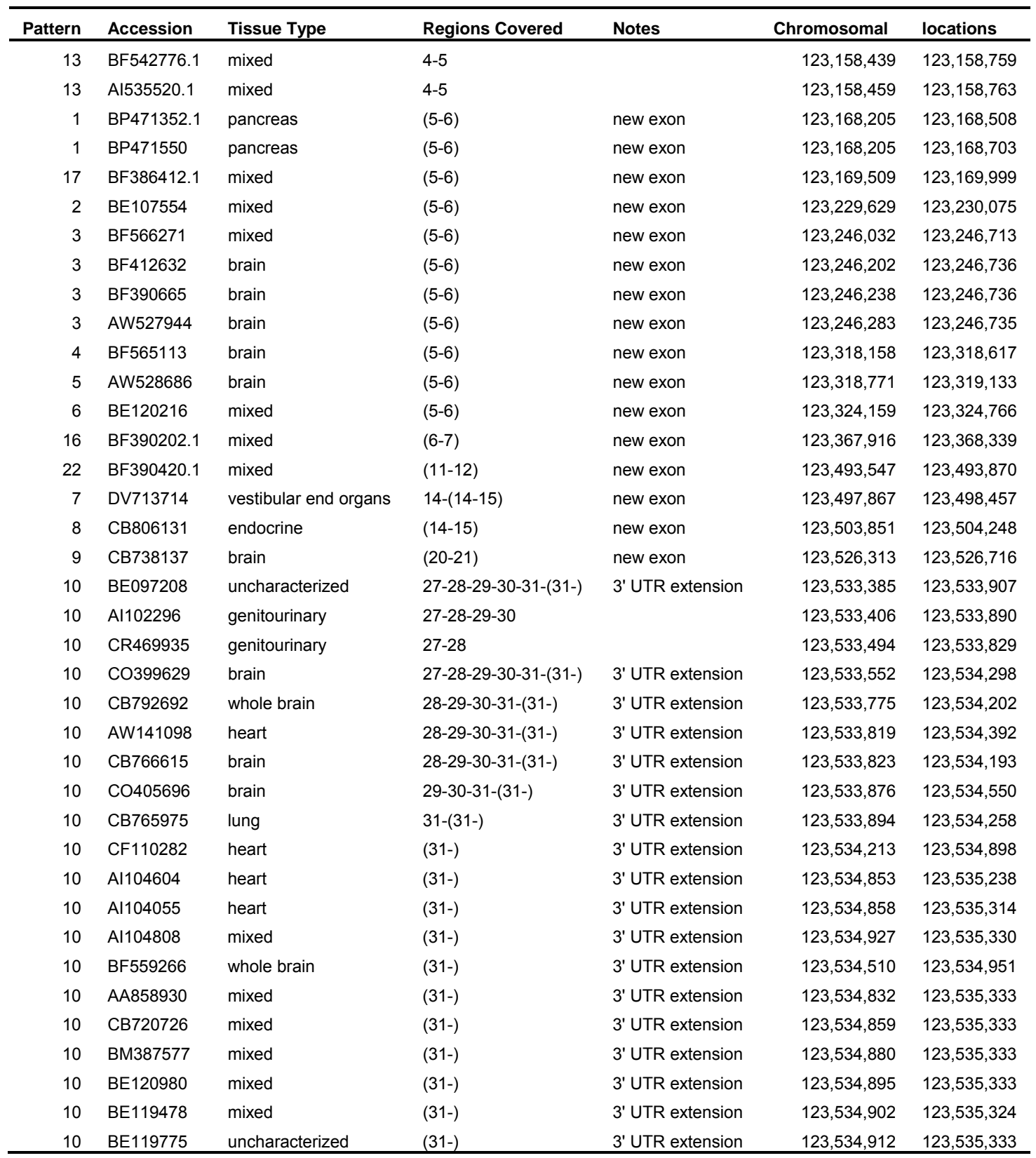


Table 4.9: Multiple Exon Splicing Patterns and Tissue Types for PDE4B

\begin{tabular}{|c|c|c|c|c|c|c|}
\hline Pattern & Accession & Tissue Type & Regions Covered & Notes & Chromosomal & locations \\
\hline \multirow[t]{3}{*}{11} & CB807697.1 & brain & $20-21-22$ & & $123,525,756$ & $123,525,915$ \\
\hline & & & & & $123,527,679$ & $123,527,778$ \\
\hline & & & & & $123,529,535$ & $123,529,598$ \\
\hline \multirow[t]{4}{*}{11} & CB773843.1 & endocrine & $20-21-22-23$ & & $123,525,822$ & $123,525,915$ \\
\hline & & & & & $123,527,679$ & $123,527,778$ \\
\hline & & & & & $123,529,535$ & $123,529,689$ \\
\hline & & & & & $123,529,781$ & $123,529,815$ \\
\hline \multirow[t]{3}{*}{12} & CB784576.1 & mixed & $5-7-8$ & & $123,158,708$ & $123,158,887$ \\
\hline & & & & & $123,416,343$ & $123,416,537$ \\
\hline & & & & & $123,426,319$ & $123,426,338$ \\
\hline \multirow[t]{5}{*}{12} & CK599899.1 & heart & $5-7-8-9-10-11$ & & $123,158,608$ & $123,158,887$ \\
\hline & & & & & $123,416,343$ & $123,416,537$ \\
\hline & & & & & $123,426,319$ & $123,426,355$ \\
\hline & & & & & $123,426,452$ & $123,426,522$ \\
\hline & & & & & $123,434,704$ & $123,434,753$ \\
\hline \multirow[t]{2}{*}{15} & BP483665.1 & pancreas & $(5-6)-(5-6)$ & $\begin{array}{l}\text { new } \\
\text { exons }\end{array}$ & $123,168,478$ & $123,169,118$ \\
\hline & & & & & $123,169,135$ & $123,169,249$ \\
\hline \multirow[t]{3}{*}{19} & CK599213..1 & heart & $13-14-15-16-17-18$ & & $123,497,581$ & $123,497,915$ \\
\hline & & & & & $123,518,627$ & $123,518,720$ \\
\hline & & & & & $123,523,794$ & $123,523,973$ \\
\hline \multirow[t]{5}{*}{19} & CV109674.1 & placenta & $13-14-15-16-17-18-19-20$ & & $123,497,681$ & $123,497,915$ \\
\hline & & & & & $123,518,627$ & $123,518,720$ \\
\hline & & & & & $123,523,794$ & $123,523,972$ \\
\hline & & & & & $123,525,516$ & $123,525,615$ \\
\hline & & & & & $123,525,753$ & $123,525,814$ \\
\hline \multirow[t]{2}{*}{20} & AA799729.1 & heart & 13-14-(31-) & $\begin{array}{l}\text { new } \\
\text { splice }\end{array}$ & $123,497,541$ & $123,497,915$ \\
\hline & & & & & $123,535,118$ & $123,535,311$ \\
\hline \multirow[t]{5}{*}{24} & CB718665.1 & brain & $(11-12)-14-16-17-18-19$ & & $123,496,114$ & $123,496,167$ \\
\hline & & & & & $123,497,803$ & $123,497,915$ \\
\hline & & & & & $123,518,627$ & $123,518,720$ \\
\hline & & & & & $123,523,794$ & $123,523,972$ \\
\hline & & & & & $123,525,516$ & $123,525,596$ \\
\hline \multirow[t]{2}{*}{23} & BF563905.1 & brain & $(14-15)-15-(15-16)-16$ & $\begin{array}{l}\text { new } \\
\text { exons }\end{array}$ & $123,517,507$ & $123,517,899$ \\
\hline & & & & & $123,518,627$ & $123,518,710$ \\
\hline \multirow[t]{2}{*}{23} & AW531150.1 & brain & $(15-16)-16-(16-17)-17$ & $\begin{array}{l}\text { new } \\
\text { exons }\end{array}$ & $123,517,692$ & $123,517,899$ \\
\hline & & & & & $123,518,627$ & $123,518,836$ \\
\hline
\end{tabular}

pattern 23), one in the linker region between UCR2 and the catalytic core (pattern 23), and one was inside the catalytic core itself (pattern 23). In addition, a novel splicing pattern of known exons was found in pattern 20 derived from heart EST tissue. 


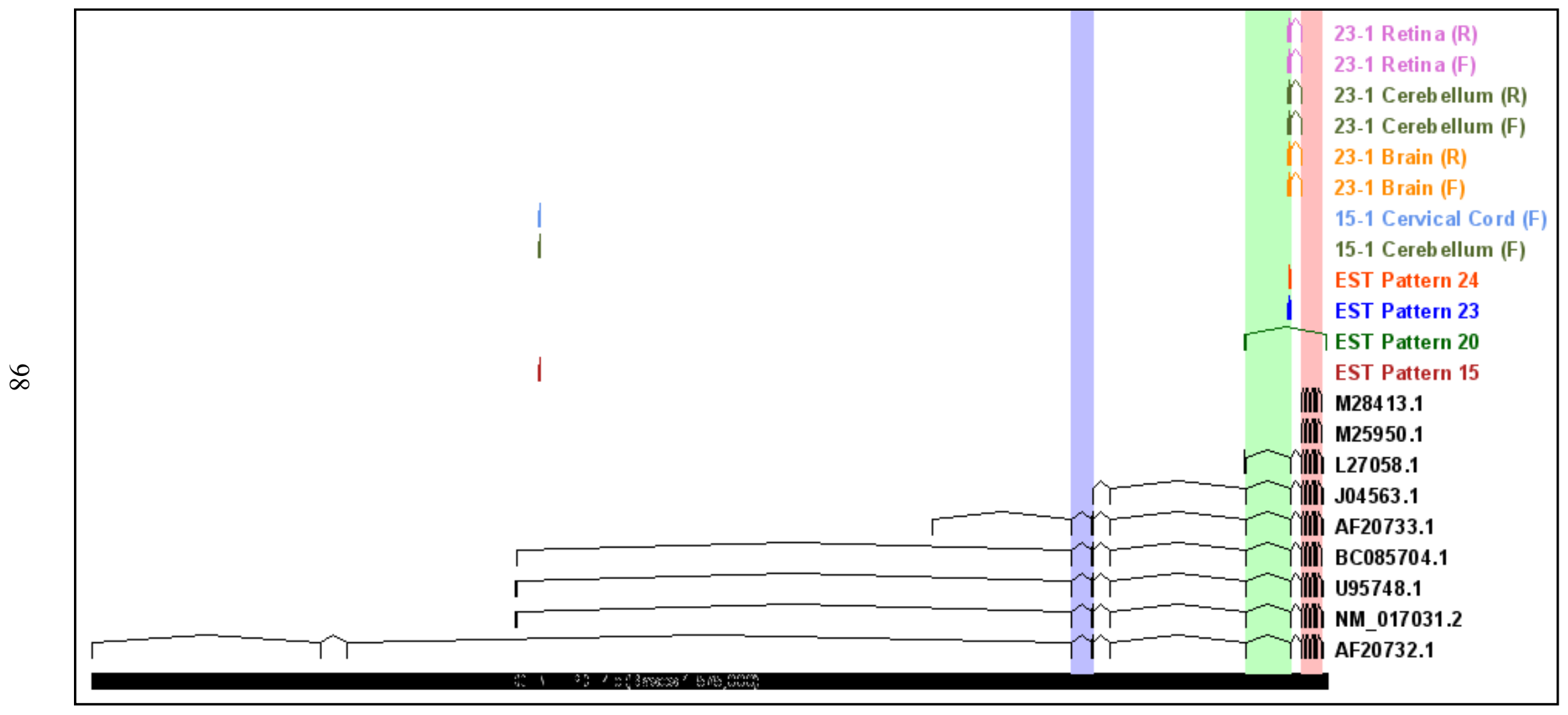

Figure 4.9: PDE4B isoforms, multiple EST patterns and sequence hits. Abbreviations: F, forward strand; $\mathrm{R}$, reverse strand. 


\subsubsection{PDE4C}

A total of 50 ESTs initially mapped to the PDE4C region. Clustering and filtering reduced this number to twelve ESTs, four representing single-exon hits and eight representing spliced exons (Table 4.10). Four of these ESTs orginate from pancreas tissue, two from insulinoma, and one each from kidney, lung and placenta. The remaining ESTs came from mixed or unknown tissue types.

A total of three separate single-exon patterns were detected. Each represented a potential novel exon between regions one and two. Seven separate multiple exon patterns appeared. Up to eleven previously uncharacterized exons between regions one and two exist, as indicated by patterns 2, 23, 24, 26, 27 and 28. In addition, there appear to be two new exons between patterns four and five (pattern 1), one between regions five and six, and one between regions six and seven (pattern 1). The new exons predicted between regions one and two occur in the N-terminal domain, while the exons predicted between regions four and five, and between five and six occur in UCR1. The exon predicted between regions six and seven occur in the linker region between UCR2 and the conserved catalytic core. The detection of a large number of potentially uncharacterized exons in the $\mathrm{N}$-terminal domain provides great insight since only three isoforms were previously described for the rat PDE4C family of genes. 


\section{Table 4.10: Splicing Patterns and Tissue Types for PDE4C}

\begin{tabular}{|c|c|c|c|c|c|c|c|}
\hline & Pattern & Accession & Tissue Type & Regions Covered & Notes & Chromosomal & locations \\
\hline \multirow[t]{4}{*}{$\begin{array}{l}\text { single } \\
\text { exon }\end{array}$} & 3 & AW921872.1 & $\operatorname{mix}$ & $(1-2)$ & new exon & $19,216,126$ & $19,216,545$ \\
\hline & 4 & BP498358.1 & insulinoma & $(1-2)$ & new exon & $19,217,290$ & $19,217,705$ \\
\hline & 4 & BP502440.1 & insulinoma & $(1-2)$ & new exon & $19,217,292$ & $19,217,839$ \\
\hline & 25 & BP484004.1 & pancreas & $(1-2)$ & new exon & $19,218,256$ & $19,218,972$ \\
\hline \multirow[t]{19}{*}{$\begin{array}{l}\text { multi } \\
\text { exon }\end{array}$} & 1 & BP471523.1 & pancreas & $(6-7)-6-(5-6)-5-(4-5)-(4-5)$ & new exons & $19,201,287$ & $19,201,766$ \\
\hline & & & & & & $19,201,883$ & $19,201,887$ \\
\hline & 1 & BP478640.1 & pancreas & $(6-7)-6-(5-6)-5-(4-5)-(4-5)$ & new exons & $19,201,335$ & $19,201,766$ \\
\hline & & & & & & $19,201,883$ & $19,201,887$ \\
\hline & 2 & Al233747.1 & kidney & $(1-2)-(1-2)$ & new exons & $19,214,941$ & $19,215,074$ \\
\hline & & & & & & $19,215,431$ & $19,215,680$ \\
\hline & 23 & CF114754.1 & lung & $(1-2)-(1-2)$ & new exons & $19,217,002$ & $19,217,281$ \\
\hline & & & & & & $19,217,832$ & $19,218,050$ \\
\hline & 24 & BM389421.1 & $\operatorname{mix}$ & $(1-2)-(1-2)$ & new exons & $19,217,276$ & $19,217,788$ \\
\hline & & & & & & $19,217,987$ & $19,218,032$ \\
\hline & 26 & BP474083.1 & pancreas & $(1-2)-(1-2)$ & new exons & $19,218,709$ & $19,218,990$ \\
\hline & & & & & & $19,219,043$ & $19,219,241$ \\
\hline & 27 & CV126227.1 & placenta & $(1-2)-(1-2)-1-(1-)$ & new exons & $19,218,107$ & $19,218,147$ \\
\hline & & & & & & $19,218,828$ & $19,219,238$ \\
\hline & & & & & & $19,220,903$ & $19,221,016$ \\
\hline & 28 & BQ200281.1 & unknown & $(1-2)-(1-2)-(1-2)-1$ & new exons & $19,217,317$ & $19,217,783$ \\
\hline & & & & & & $19,219,088$ & $19,219,196$ \\
\hline & & & & & & $19,219,201$ & $19,219,238$ \\
\hline & & & & & & $19,220,903$ & $19,220,985$ \\
\hline
\end{tabular}

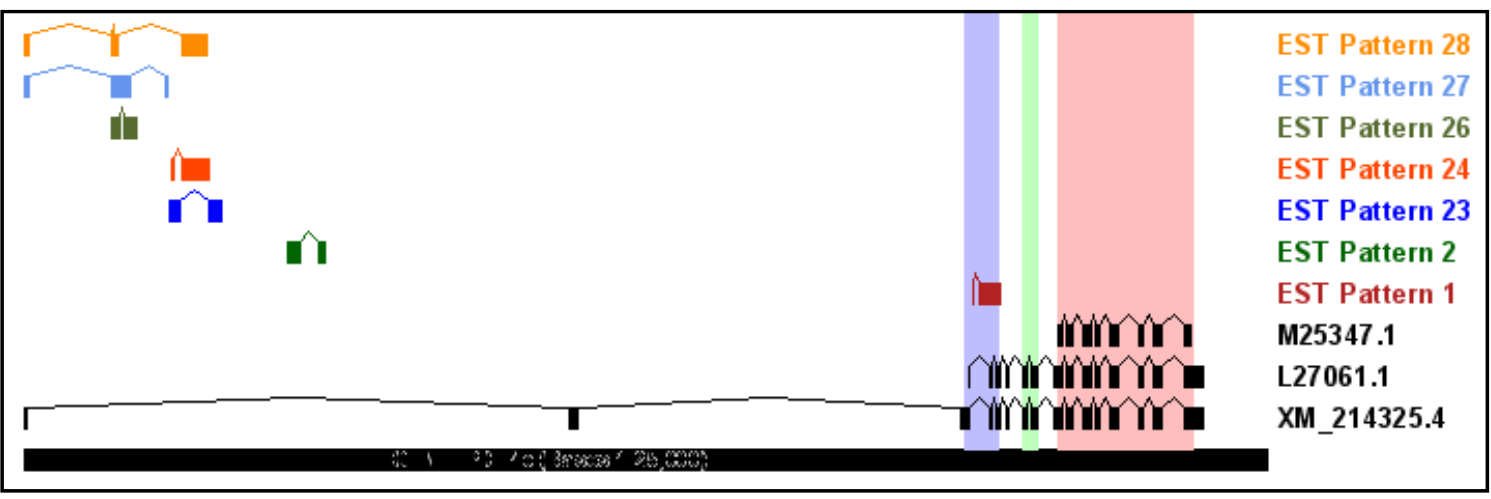

Figure 4.10: PDE4C isoforms, multiple EST patterns and sequence hits. 


\subsubsection{PDE4D}

PDE4D had an inordinately large number of EST hits $(38,742)$. However, most of these fell into a single exonic region that appeared to be a previously uncharacterized rat repetitive element. Once these hits are removed, 18 single-exon and three multiple-exon hits remain (Table 4.11).

The 18 single-exon hits produced 15 unique patterns, each of which suggested novel exons. Up to seven novel exons were detected between regions five and six (patterns 1, 2, 3, 4, 5 and 22) with an additional five between regions six and seven (patterns 6, 7, 8 and 9), three between regions 12 and 13 (patterns 11, 12 and 13), and one each between regions eight and nine (pattern 10) and between regions 17 and 18 (pattern 14).

The three spliced EST hits create three separate patterns. Pattern 21 suggested two new exons, one between regions six and seven, and one between regions seven and eight. Pattern 16 suggested a single novel exon between regions 12 and 13, while pattern 17 revealed an exon between regions 20 and 21.

Fourteen of the predicted exons occurred in the 5 , N-terminal domain (as indicated from patterns $1,2,3,4,5,6,7,8,9,21$ and 22), one was in the UCR1 region (pattern 10), five were in the linker region between UCR1 and UCR2 (patterns 11, 12, 13 and 16), one was in the UCR2 region (pattern 14), and one is in the catalytic core (pattern 17). 


\section{Table 4.11: Splicing Patterns and Tissue Types for PDE4D}

\begin{tabular}{|c|c|c|c|c|c|c|c|}
\hline & Pattern & Accession & Tissue Type & Regions Covered & Notes & Chromosomal & locations \\
\hline \multirow[t]{18}{*}{$\begin{array}{l}\text { single } \\
\text { exon }\end{array}$} & 1 & CF106982.1 & lung & $(5-6)$ & new exon & $40,821,601$ & $40,822,129$ \\
\hline & 2 & CB735250.1 & brain & $(5-6)$ & new exon & $40,844,702$ & $40,845,000$ \\
\hline & 3 & BF561343.1 & mixed & $(5-6)$ & new exon & $40,928,435$ & $40,928,658$ \\
\hline & 3 & AA99742.1 & mixed & $(5-6)$ & new exon & $40,928,541$ & $40,929,191$ \\
\hline & 4 & CR471157.1 & uncharacterized & $(5-6)$ & new exon & $40,938,872$ & $40,939,138$ \\
\hline & 22 & CF114850.1 & lung & $(5-6)$ & new exon & $40,962,055$ & $40,962,763$ \\
\hline & 5 & CR460313.1 & uncharacterized & $(5-6)$ & new exon & $40,988,404$ & $40,988,701$ \\
\hline & 6 & BF567734.1 & brain & $(6-7)$ & new exon & $41,018,068$ & $41,018,402$ \\
\hline & 6 & AW522698.1 & brain & $(6-7)$ & new exon & $41,018,068$ & $41,018,471$ \\
\hline & 7 & CF107424.1 & lung & $(6-7)$ & new exon & $41,055,789$ & $41,056,469$ \\
\hline & 8 & BG379252.1 & brain & $(6-7)$ & new exon & $41,060,267$ & $41,060,631$ \\
\hline & 9 & AW434715.1 & heart & $(6-7)$ & new exon & $41,063,099$ & $41,063,498$ \\
\hline & 10 & BF401594.1 & mixed & $(8-9)$ & new exon & $41,119,841$ & $41,120,456$ \\
\hline & 11 & BQ192875.1 & uncharacterized & $(12-13)$ & new exon & $41,145,546$ & $41,145,899$ \\
\hline & 12 & CF113676.1 & lung & $(12-13)$ & new exon & $41,272,381$ & $41,272,835$ \\
\hline & 13 & BG377764.1 & genitourinary & $(12-13)$ & new exon & $41,274,996$ & $41,275,383$ \\
\hline & 13 & BE118751.1 & mixed & $(12-13)$ & new exon & $41,274,997$ & $41,275,392$ \\
\hline & 14 & BE103213.1 & brain & $(17-18)$ & new exon & $41,284,770$ & $41,285,306$ \\
\hline \multirow[t]{11}{*}{$\begin{array}{l}\text { multi } \\
\text { exon }\end{array}$} & 16 & BP469236.1 & pancreas & $11-12-(12-13)-17-19-20$ & new exon & $41,136,455$ & $41,136,512$ \\
\hline & & & & & & $41,139,162$ & $41,139,211$ \\
\hline & & & & & & $41,153,803$ & $41.153,963$ \\
\hline & & & & & & $41,275,484$ & $41,275,548$ \\
\hline & & & & & & $41,275,555$ & $41,275,596$ \\
\hline & & & & & & $41,305,886$ & $41,305,979$ \\
\hline & & & & & & $41,307,257$ & $41,307,301$ \\
\hline & 17 & BF566977.1 & heart & $19-20-(20-21)$ & new exon & $41,305,945$ & $41,305,979$ \\
\hline & & & & & & $41,307,257$ & $41,307,451$ \\
\hline & 21 & CB322599.1 & cartilage & $(6-7)-7-(7-8)$ & new exon & $41,063,589$ & $41,063,737$ \\
\hline & & & & & & $41,065,963$ & $41,066,486$ \\
\hline
\end{tabular}




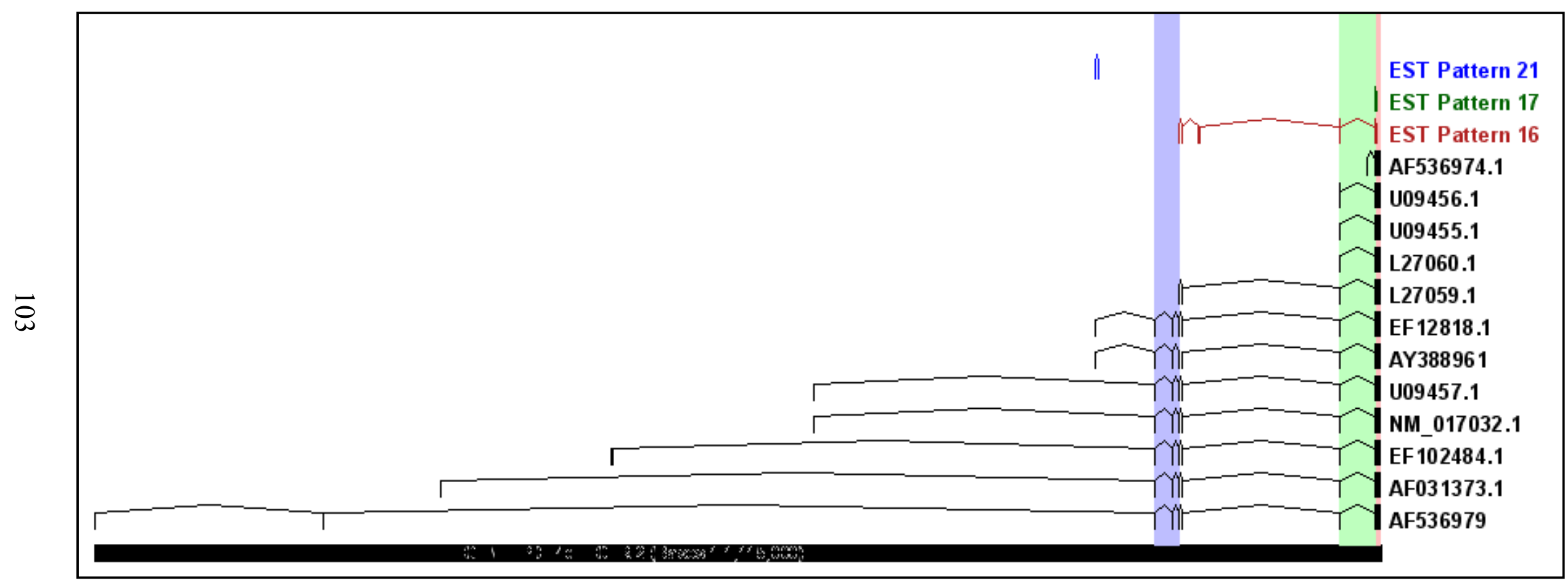

Figure 4.11: PDE4D isoforms, multiple EST patterns and sequence hits (chromosome 2). 


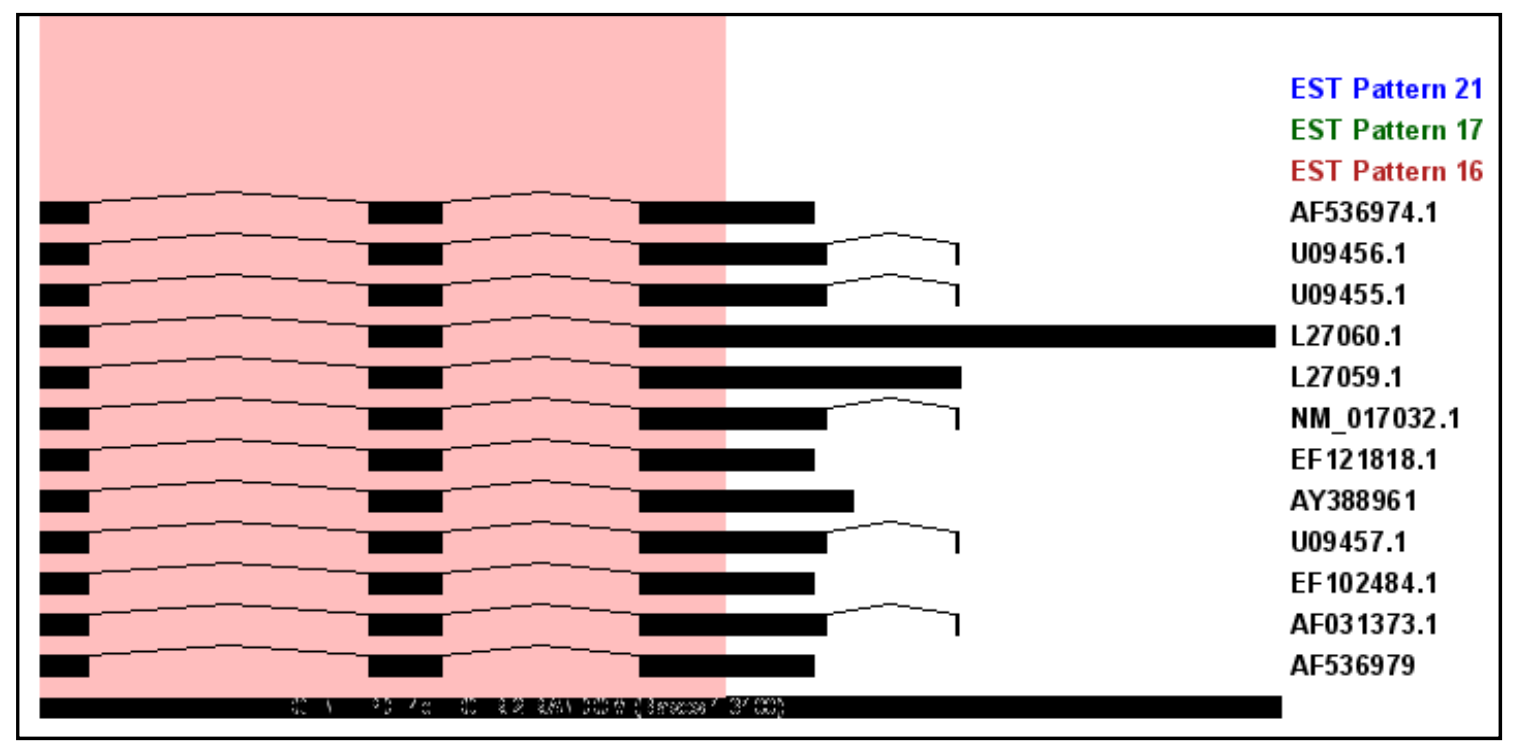

Figure 4.12: PDE4D isoforms, multiple EST patterns and sequence hits (chromosome 2 RANDOM).

\subsection{Wet Lab Methods}

Select sequence patterns found by computational methods were further tested for verification in the wet lab using reverse transcriptase polymerase chain reaction (RTPCR) and DNA sequencing.

\subsubsection{Polymerase Chain Reaction (PCR)}

PCR, or polymerase chain reaction, amplifies specific DNA segments by exploiting certain features of DNA replication in vitro ${ }^{24}$. The method was developed by the Cetus ${ }^{25}$ scientist Kary Mullis in 1983 (171;172). The PCR technique won Mullis a shared Nobel Prize in Chemistry "for his invention of the polymerase chain reaction

\footnotetext{
${ }^{24}$ In vitro is a test performing in glass or plastic vessels in the laboratory.

${ }^{25}$ Cetus Corporation was one of the first biotechnology companies. It was established in Berkeley, California in 1971. Cetus Corp. developed several significant pharmaceutical drugs as well as a revolutionary DNA amplification technique. Cetus Corp. was merged with Chiron Corporation in 1991 and Chiron Corp. was further integrated into Novartis Pharmaceuticals (http://www.novartis.com/) in 2006.
} 
(PCR) method," along with Michael Smith "for contribution to the developments of methods within DNA-based chemistry."

The PCR technique uses a pair of primers, which are DNA segments approximately $20 \mathrm{bp}$ long that complement to the DNA being synthesized and a thermo stable DNA polymerase to achieve near-exponential enzymatic amplification of target DNA. PCR proceeds in three stages: denature double-stranded DNA, anneal primer, and extend the annealed primers (171-174). Figure 4.13 shows the basic PCR cycle.

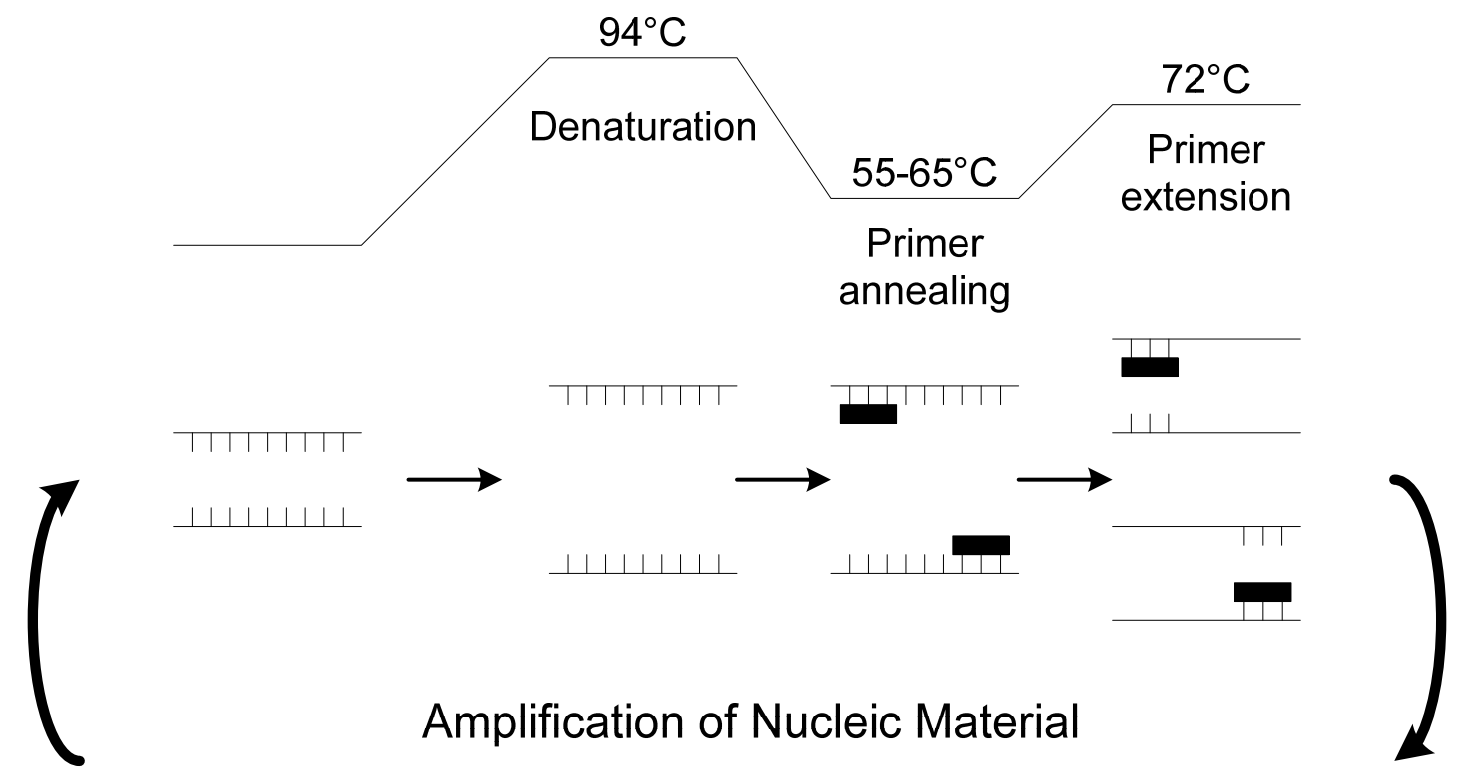

Figure 4.13: Basic steps in the PCR cycle.

Hatched lines indicate target DNA. Filled boxes represent annealed primers.

\subsubsection{RT-PCR}

RT-PCR, abbreviated for reverse transcriptase polymerase chain reaction, is a highly sensitive technique for the detection and quantization of mRNA. This technique includes two parts: the synthesis of cDNA from RNA by reverse transcript and the amplification of a specific cDNA by the polymerase chain reaction. 


\subsubsection{DNA Sequencing}

Sequences which had been amplified by the RT-PCR method were extracted and run through DNA sequencing. If the target sequences found from computation matched the sequences from the wet lab, it would highly to be verified the presence of novel exons and which had not been reported.

\subsubsection{Splice Exon Verification}

The novel exons predicted from computation with a length of at least 25 bases and a sequence similarity of at least $98 \%$ to the genomic regions were studied using RT-PCR and DNA sequencing techniques. PCR primer pairs were detected using MPrime 1.3 (175). Since each of the predicted exons were located upstream of the catalytic core which should be expressed in all viable variants, one primer was localized within the predicted exon and the other anchored inside of the catalytic core. A primer min, max, and opt size of $20 \mathrm{bp}$ was incorporated with a primer $\mathrm{GC} \%{ }^{26} \min$ of $30 \%$, $\max$ of $60 \%$ and opt of $50 \%$. The melting temperature was set to a minimum of 57 , max of 63 , and optimum of 60 . A product minimum size of 395 bases was used with a maximum of 405 and an optimum of 400 , except for those cases where one primer could not be found anchored in the predicted exon. For those cases, a min value of 195 was used with a max of 205 and an opt of 200. A GC clamp of 2 was incorporated.

${ }^{26} \mathrm{GC} \%$ represents the percentage of total number of G's and C's in the sequence. For instance, GC\% $30 \%$ means that the number of G's and C's occur $30 \%$ in the sequence 


\subsection{Results in Verified Predicted Exons}

\subsubsection{Tissue Types}

The tissue types were found for the splice patterns in each PDE4 gene in computational methods were sequenced using RT-PCR. Tissues from rat liver, lung, sciatic nerve, testes, heart, brain (cortex), cerebellum, spinal cord, skeletal muscle, spleen, optic nerve, kidney, small intestine, retina, aorta and seminal vesicle were extracted and verified.

\subsubsection{Predicted Exons Verification}

In order to validate the proposed computational approach as an accurate predictor of novel isoforms, three novel splice patterns showing the potential for tissue specificity were chosen for further study. These included pattern 14 from PDE4A, patterns 15 and 23 from PDE4B, and patterns 21 and 22 from PDE4D. PCR primers were designed using MPrime (175). (See Section 4.7.4). All primers (Shown in Table 4.12) were then used to perform RT-PCR on RNA extracted from rat liver, lung, sciatic nerve, testes, heart, brain (cortex), cerebellum, spinal cord, skeletal muscle, spleen, optic nerve, kidney, small intestine, retina, aorta and seminal vesicle. 


\section{Table 4.12: PCR Primer Pairs for Selected Predicted Exons}

\begin{tabular}{cccc}
\hline Gene Family & Pattern & Forward Primer & Reverse Primer \\
\hline PDE4A & $14-1$ & CCAGAACCATCCCTAAGACC & TGTCGCAGTTCTCTTCTTGC \\
PDE4B & $15-1$ & GTGTGCTCTGGAATTTTTGG & TACATGATGCATGTGAGGGG \\
PDE4B & $23-1$ & ATAAGCAGGGAGCCCATAGG & GACTCCAAAGCGTGAGATGC \\
PDE4D & $21-1$ & CACAACAACATCCATGCTGC & TCACCTTCTTCGTTTCAACC \\
PDE4D & $21-2$ & CTCCTTATGCCACTGACACG & CACAGTTTTCTTCCTGGAGC \\
PDE4D & $22-1$ & TCAGTCCAAGAAGATTGCCG & CAGCCAGCAGATTCATGTGC \\
PDE4D & $22-2$ & CACAACAACATCCATGCTGC & TCACCTTCTTCGTTTCAACC \\
\hline
\end{tabular}

Reverse transcriptase PCR results indicate that PDE4A pattern 14 is tissue specific to testes with a single band occurring at approximately $900 \mathrm{bp}$ (Figure 4.14). PDE4B pattern 23 is expressed in brain cortex, cerebellum and retina with a strong band between 500 and $600 \mathrm{bp}$ and additional two lighter bands appearing for brain and cerebellum (Figure 4.15, lower panel). PDE4B pattern 15 appears for cerebellum and spinal cord at approximately 300 bp (Figure 4.15, upper panel). PDE4D patterns 21 and 22 show very strong banding patterns at 400 bp for every tissue type, with the exception of seminal vesicle for pattern 22 (Figure 4.16). The results for the PDE4D patterns were expected since the PCR primers were designed within the conserved catalytic core. 


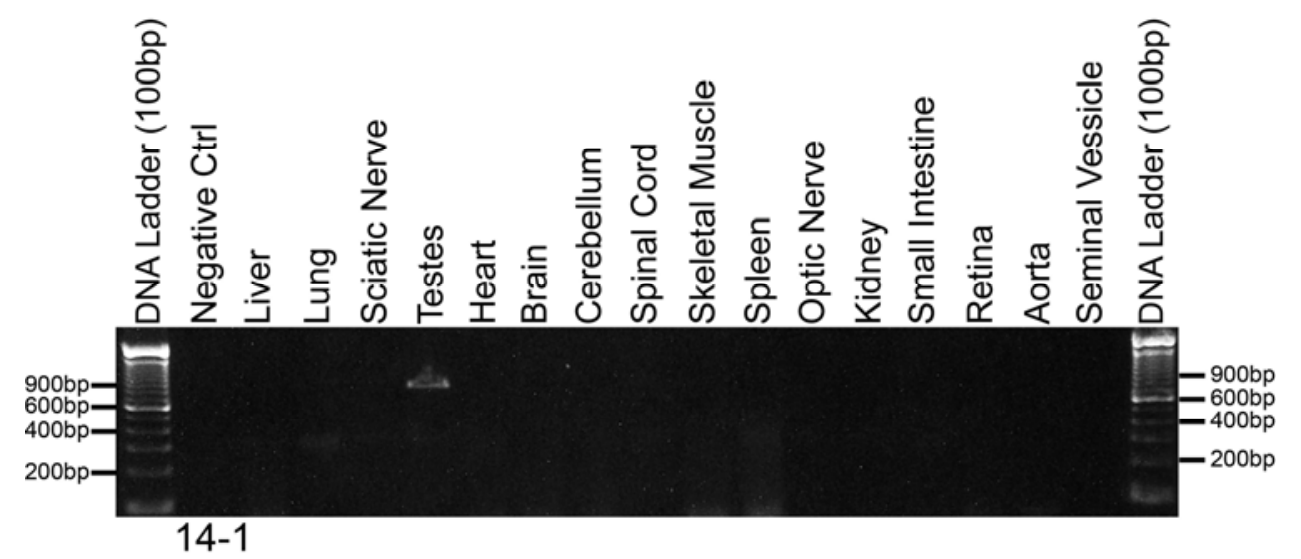

Figure 4.14: RT-PCR for PDE4A pattern 14.

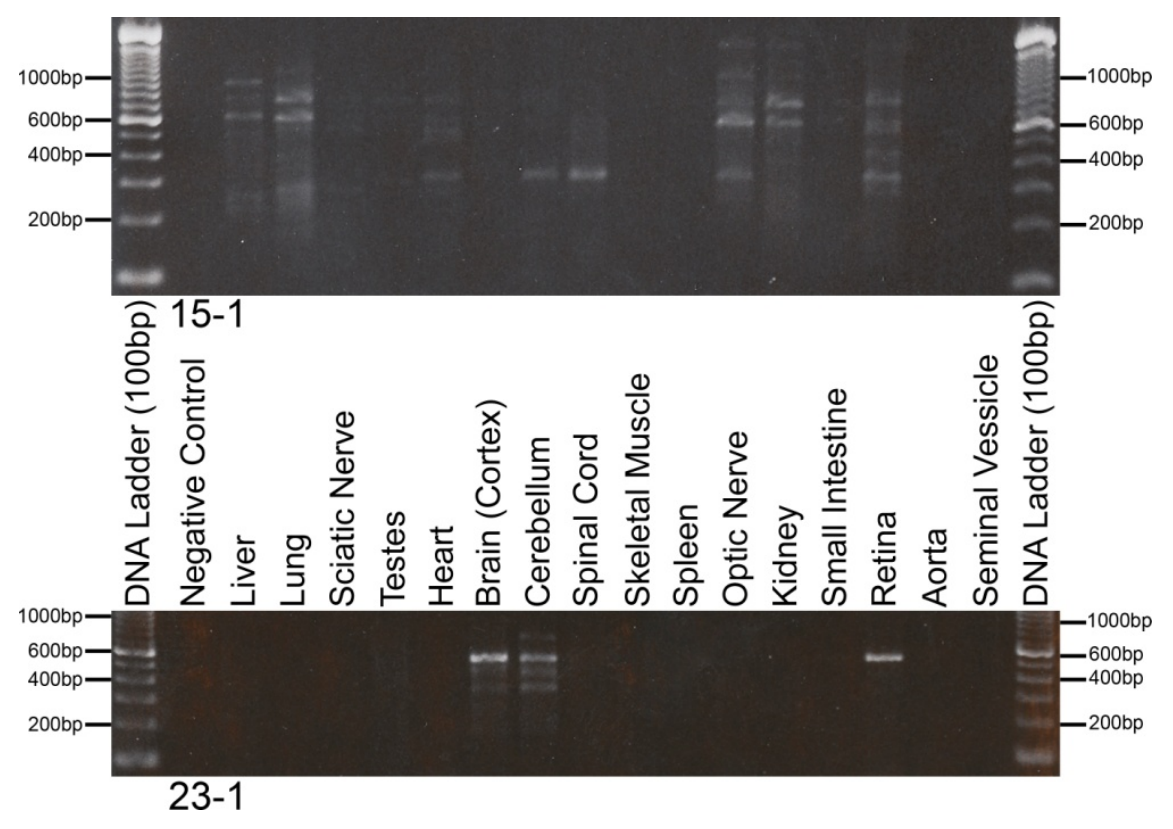

Figure 4.15: RT-PCR for PDE4B patterns 15 and 23. 


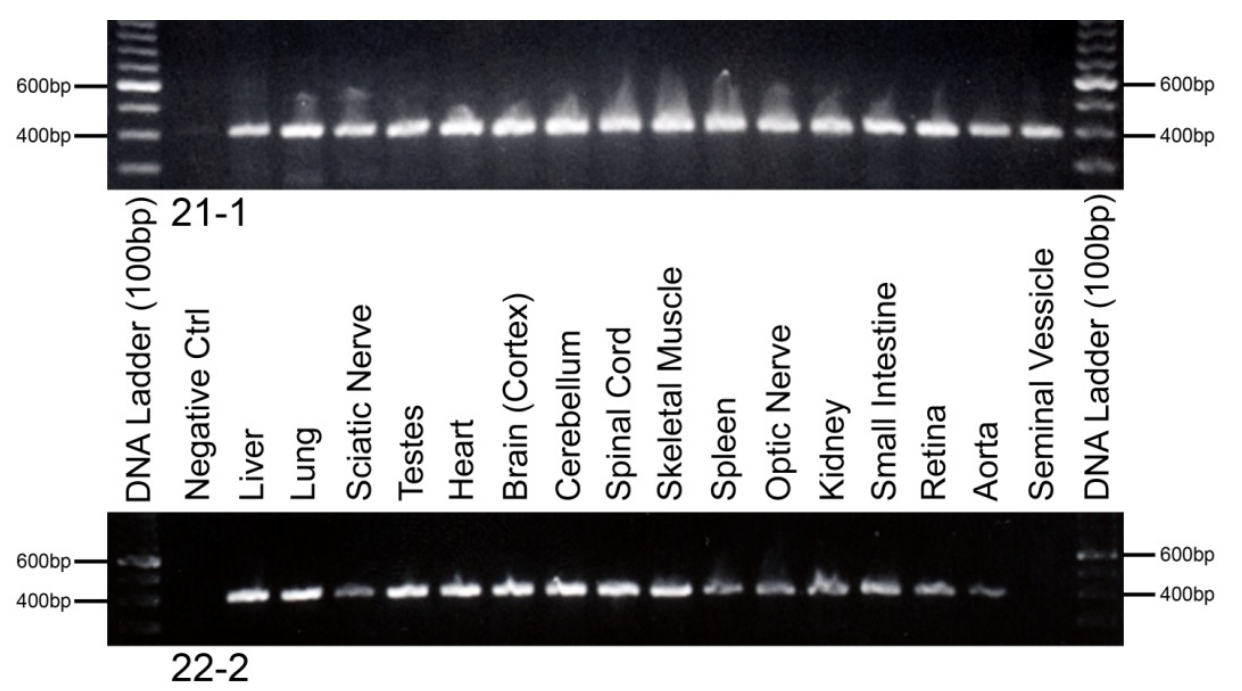

Figure 4.16: RT-PCR for PDE4D patterns 21 and 22.

Next, DNA sequencing was used to confirm that the observed bands corresponded to the gene region of interest using the forward and reverse PCR primers as independent sequencing primers (See Section 4.7.4). The resulting sequences are shown in Tables 4.13-4.15.

For PDE4A pattern 14, the band found in testes corresponded to the PDE4A region of chromosome 8. Mapping of the sequence is shown in Table 4.2 and Figure 4.3. The sequencing confirmed additional exons for PDE4A pattern 14 that were not found in any known isoforms.

Two patterns were considered for PDE4B. The resulting sequence maps are provided in Table 4.3 and Figure 4.4. For PDE4B pattern 15, two bands were extracted and sequenced: the cerebellum and spinal cord bands occurring at $300 \mathrm{bp}$. Pattern 23 had three bands extracted and sequenced, for the brain, cerebellum, and retina. Sequencing confirmed that all of these bands were unique to PDE4B and revealed new exons previously uncharacterized for PDE4B isoforms. 
The strong banding patterns for patterns 21 and 22 of PDE4D were extracted and sequenced as well (results not shown). The resulting sequences map uniquely back onto the catalytic region for PDE4D.

Table 4.13: Sequencing Results (Primer 14-1 in PDE4A)

>14-1_PDE4A_TestesForwardPrimer
AGGAAAGTACTTACATGTGTCCCATGTTTCAAACCTCAGAGAAGAGACCAGCATTGCCGTC
CGCAGGATGACCTCATATCAAACAAGCAGAATGAAGTGGAGATCCCTCACCCACACCTCGG
CAGAGAGCCTTCCAGCAGCCCCCGCCGTCAGTGCTGCGACAGTCCCAGCCCATGTCTCAGAT
CACAGGGCTGAAAAAGTGGTACACACTGGAAGCTTGAACACCAACGTCCCACGGTTTGGAG
TCAAGACAGATCAAGAGGACCTCTTAGCACAAGAACTGGAGACTTGAGCAAATGGGGCCTG
AACATCTTTTGTGTTCGGAGTACGCTGGAGGCCGCTCACTCAGCTGTATCATGTATACAAT
ATTCCAGGAGCGGGCCTACTGAAGAAATTCCACATCCCTGTGGACACCATGATGATGTACA
TGCTGACCCTGGAGGACCACTACCATGCCGACGTGGCCTACCACAACAGCCTGCACGCAGCG
GATGTGCTGCAGTCCACACACGTGCTGCTGGCCACGCCCGCACTGGACGCTGTGTTCTACTG
ACCTGGAGATTCTTGCTGCCCTCTTCGCTGCTGCCATCCACGATGTGGACCACCCTGGCGTC
TCCACCCAGTTCCTAATCAACACCAATTCGGAGCTGGCGTTGATGTACAACAATGAGTCTGT
GCTTGGAAAACCACCACCTGGGCTGTGGGGATCCAAGCCGCCCCAAAAAAAAAAACCTGCAA
AACANGAANACTCNGTGGNGGNN
>14-1_PDE4A_TestesReversePrimer
GGTGGTGGTTCTCAAGCACAGACTCATCGTTGTACATCAACGCCAGCTCCGAATTGGTGTTG
ATTAGGAACTGGTTGGAGACGCCAGGGTGGTCCACATCGTGGATGGCAGCAGCGAAGAGGGC
AGCAAGAATCTCCAGGTCTGTGAACACAGCGTCCAGTGCGGGCGTGGCCAGCAGCACGTGTG
TGGACTGCAGCACATCCGCTGCGTGCAGGCTGTTGTGGTAGGCCACGTCGGCATGGTAGTGG
TCCTCCAGGGTCAGCATGTACATCATCATGGTGTCCACAGGGATGTGGAATTTCTTCAGTAG
GTCCCGCTCCTGGAATATCGTATACATGATACAGCTGAGTGAGCGGCCTCCAGCGTACTCCG
ACACACAAAAGATGTTCAGGCCCCATTTGGCTCAAGTCTACTCCAGTTCTTGTGCTAAGAGG
TCCTCTTGATCTGTCTTGACTCCAAACCGTGGGACGTTGGTGTTCAAGCTTCCAGTGTGTAC
CAGCTTTTCAGCCCTGTGATCTGAGACATGGGCTGGGACTGTCNCACCACTGACGGCGGGG
GCTGCTGGAAGGCTCTCTGCCAAGGTGGGGTAGGGATCCCCCACTTCATTTCTGCTTGT
TTTGATATTAAAGGTCTTCCCTGCCGGACNGCAAATGCCTGGTCTTCTTCCTCTAAAGGGTT
TGAAACCTGGGACCCCTTNTAAAAAACCTTTCCTGGGAAGGTGGGTGGCCCCCCCTTGG
GGGTCTTAAGGGATGGTTATGGGGA


Table 4.14: Sequencing Results (Primer 15-1 in PDE4B)

>15-1_PDE4B_CerebellumForwardPrimer

TGTACATGAAGTTTTATTCTAATTTGTAAGAATGTGTCTACCTATCTAACATGGCAAGCATCT CTCTATGGGATNACAGTATGTGGCAGAGAGTATGATGTCACATAGATGTGCTTAATGTCTCTA TCCAATCTCTACACACACACACACACACACACACACACACACACTCTTTTTCTACTATCATCA TGAGAGATATATATCACATGATGATAAATAATACTAGTAGTAACTTATATCTACTCTATCATC ATCACCTACCTCCTCTCGCTCGCTCGTTCCTCTATCATCATCACCGCCCCTCCTCTCATCGAT CGTCGTCCCCTATCTATCGTCGTCGATCTATATAGATAGATAAAGAGGGCGTGGGCCCTTTCA TTTAGATGGAGGAGGGGGGGGGGGTATGGGGGGGAGTTTAGTAAGTTGTGTATGGGATGAAAA GAAACTAACCCACAGTATGGTAGGGCATTGTAGGTGAAAGGAAAAATAGTNNANNNNNNNNNN NNNNATAA

>15-1_PDE4B_CerebellumReverse

TGGAACGAAGGAACAGTGTAAATGAAGTACTNGTCATTTGCATTTTCAAGCTAGTCATTCAAA ATAGACAAAGACAACAGATGTGTGTGTGTGTGTGTGTGTGTGTGTGTGTGTAGTATATATACG ATCATCATCATCATCAGCAGTCATCACTACTGTACTTCATCAGTCCATTCATNCATGCTCGCC GCACCTTCATCATCCTNGTCTCCTCCACTAACGATGACCATCATCGCCGCCTGGTCGTAATTA GCTGAGTAGTAGCAGCATCATCTTCTGCTATAANATAATNATACTACTACTCACTCGTAGGTC AGTCAGTCAGTCAGTCAGTCAGTCAGTGATGTGTGTGTGTCAGNCAGCTCAGACACATTANAC TCACTCACACCCACCTGACTGAATTCATNCAACAACTTACTTCCTTCTGCCCTACGATCGTGG TCGTTGACTGATGATGTCTATCATTNAGTAGTCATGATTGAAAGAAGATTGACTTACTACAAA ATATTATTTATTTTATCAAAAGAAGNCAACAACTNCANGCAGCTAGCAGTTAGTTANGTAAGT AACANACACGACGAGCCTGANAATCAATTTATATNTATANCAGGTCAATACGNCAATCACGTN ATTCATGAATTCCATNAAGA

>15-1_PDE4B_CervicalCordForwardPrimer

TCATGTACATTAAGTTTTATTTTAATTTTAAGAATGTGTCTACCTATCTAACATGGCAAGCAT CTCTCTATTGGATTACAGTATGTGGCAGAGAGTATGATGTCACATAGATGTGCTTAATGTCTC TATTCAATCTCTACACACACACACACACACACACACACACACACACTCTTTTTCTACTATTCA TCATGTAGAAGAATATATATCAATCCATGCATATATATAAATAAATACCNAGATAGATAACAT AAATATCTACCTCTATCAATCAATCAGCTACCTCTTCTCGCTCGCTCGCTCTCTCTATCATCA TCACCGTCCTCCTCTCGATCGATCGTCGCTCCTCTACTATCGATCGATCGATCTATATAGATA GATAAGAAAGAGGGGCGCGGGGCCCCCCCGGTGGCGGAGGGGGGGNCGGGGGGGGTGAAA 
Table 4.15: Sequencing Results (Primer 23-1 in PDE4B)

>23-1_PDE4B_BrainForwardPrimer

TATCNTAGCAATGAATAGAGTATGAACGGAGATGGGCTTGCTGTGGATTAGCAAATACCGCCC ATTTGGAAGGCTTTGTCTTTTCTAAGTTTTGGTTCTTTAACCACCGGAGCTGAGAGCTGCGGA GGTTTCCTGATCCTCTGGAGACCGCTCTTACAGAACTGAATTCTTTCAAAGGGATTTGTGGAT TGTGGCAAGAAGAGCTTCCAAAATGCCTGAGGCAAACTATTTGTTATCTCTATCTTGGGGTTA TATCAAGTTCAAAAGGATGCTAGAACCGGGAGCTGACACACCTCTCAGAGATGAGCAGATCAG GGAACCAAGTGTCTGAATACATTTCGAACACGTTCTTAGACAAGCAGAACGATGTGGAAATCC CATCTCCCACCCAGAAGGACAGGGAGAAGAAGAAGAAGCAGCAGCTCATGACCCAGATAAGTG GAGTGAANAAACTGATGCACAGCTCAAGCCTGAACAACCACAAGCATCTCACGCTTA

>23-1_PDE4B_BrainReversePrimer

TNGNACTGATGTAATTATACGGCTTGAGCTGTAGCATCAGTTTCTTCACTCCACTTATTGCTA GGGTCATGAGCTGCTGCTTCTTCTTCTTCTCCCTGTCCTTCTGGGTGGGAGATGGGATTTCCA CATCGTTCTGCTTGTCTAAGAACGTGTTCGAAATGTATTCAGACACTTGGTTCCCTGATCTGC TCATCTCTGAAGAGGTGTGTCAGCTCCCGGTTCAGCATCCTTTTGAACTTGATATAACCCCAA GATAGAGATAACAAATAGTTTGCCTCAGGCATTTTGGAAGCTCTTCTTGCCACAATCCACAAA TCCCTTTGAAAGAATTCAGTTCTGTAAGAGCGGTCTCCAGAGGATCAGGAAACCTCCGCAGCT CTCAGCTCCGGTGGTTAAAGAACCAAAACTTAGAAAAGACAAAGCCTTCCAAATGGGCGGTAT TTGCTAATCCACAGCAAGCCCATCTCCGTTCATACTCTATTCTTGCAAGAAGCCTATGGGCTC CC

>23-1_PDE4B_CerebellumForwardPrimer CNGCTATGCTAGGCTTTGATCAGAGTATGAACGGAGATGGGCTTGCTGTGGATTAGCAAATAC CGCCCATTTGGAAGGCTTTGTCTTTTCTAAGTTTTGGTTCTTTAACCACCGGAGCTGAGAGCT GCGGAGGTTTCCTGATCCTCTGGAGACCGCTCTTACAGAACTGAATTCTTTCAAAGGGATTTG TGGATTGTGGCAAGAAGAGCTTCCAAAATGCCTGAGGCAAACTATTTGTTATCTCTATCTTGG GGTTATATCAAGTTCAAAAGGATGCTGAACCGGGAGCTGACACACCTCTCAGAGATGAGCAGA TCAGGGAACCAAGTGTCTGAATACATTTCGAACACGTTCTTAGACAAGCAGAACGATGTGGAA ATCCCATCTCCCACCCAGAAGGACAGGGAGAAGAAGATAAAGCAGCAGCTCATGACCCAGATA AGTGGAGTGAANAACCTGATGCACAGCTTCATGCCTGAACAACCACAAGCTTCTCCCGCNAAA GAATAA

>23-1_PDE4B_CerebellumReversePrimer

TACTGTAGGGTANTTATAGCGCTTGAGCTGTAGCTCAGGTTTCTTCACTCCACTTATCTAGGG TCATGAGCTGCTGCTTCTTCTTCTTCTCCCTGTCCTTCTGGGTGGGAGATGGGATTTCCACAT CGTTCTGCTTGTCTAAGAACGTGTTCAAAATGTATTCAGACACTTGGTTCCCTGAATCTGCTC ATCTCTGAAGAGGTGTGTCAGCTCCCGGTTCAGCATCCTTTTAGAACTTGAATATAACCCCAA GATAGAGATAACAAATAGTTTGCCTCAGGCATTTTGGAAGCTCTTCTTGCCACAATCCACAAA TCCCTTTGAAAAGAATTCAGTTCTGTAAGAGCGGTCTCCAGAGGATCAGGAAACCTCCGCAGC TCTCAGCTCCGGTGGTTAAAGAACCAAAACTTAGAAAAGACAAAGCCTTCCAAATGGGCGGTA TTTGCTAATCCACAGCAAGCCCATCTCCGTTCATACTCTATTCTTGCAAGAAGCCTATGGGCT $\mathrm{CCC}$

>23-1_PDE4B_RetinaForwardPrimer

AATCTAAGANCTTTAGACATATAGGAACGGAGTAGGGCTTGCTGTGGATTAGCAAATACCGCC CATTTGGAAGGCTTTGTCTTTTCTAAGTTTTGGTTCTTTAACCACCGGAGCTGAGAGCTGCGG AGGTTTCCTGATCCTCTGGAGACCGCTCTTACAGAACTGAATTCTTTCAAAGGGATTTGTGGA TTGTGGCAAGAAGAGCTTCCAAAATGCCTGAGGCAAACTATTTGTTATCTCTATCTTGGGGTT ATATCAAGTTCAAAAGGATGCTAAACCGGGAGCTGACACACCTCTCAGAGATGAGCAGATCAG 


\begin{tabular}{|l}
\hline GGAACCAAGTGTCTGAATACATTTCGAACACGTTCTTAGACAAGCAGAACGATGTGGAAATCC \\
CATCTCCCACCCAGAAGGACAGGGAGAAGAAGAAGAAGCAGCAGCTCATGACCCAGATAAGTG \\
GAGTGAAGAAACTGATGCACAGCTCAAGCCTGAACAACACAAGCATCTCACGCTTA \\
>23-1_PDE4B_RetinaReversePrimer \\
TTTTCGGCTTGAGTGTAGCATCAGTTTCTTCACTCCACTTATCTAGGGTCATGAGCTGCTGC \\
TTCTTCTTCTTCTCCCTGTCCTTCTGGGTGGGAGATGGGATTTCCACATCGTTCTGCTTGTCT \\
AAGAACGTGTTCGAAATGTATTCAGACACTTGGTTCCCTGATCTGCTCATCTCTGAAGAGGTG \\
TGTCAGCTCCCGGTTCAGCATCCTTTTGAACTTGATATAACCCCAAGATAGAGATAACAAATA \\
GTTTGCCTCAGGCATTTTGGAACTCTTCTTGCCACAATCCACAAATCCCTTTGAAAAGATT \\
CAGTTCTGTAAGAGCGGTCTCCAGAGGATCAGGAACCTCCGCAGCTCTCAGCTCCGGTGGTT \\
AAAGAACCAAAACTTAGAAAAGACAAAGCCTTCCAAATGGGCGTATTTGCTAATCCACAGCA \\
AGCCCATCTCCGTTCATACTCTATTCTTGCAAGAAGCCTATGGGCTCCCTGCC \\
\hline
\end{tabular}

\subsection{Discussion}

Computational mapping of publicly available rat ESTs to genomic regions for the PDE4A, PDE4B, PDE4C and PDE4D genes yields more than 25 previously unreported exonic regions. In actuality, the number may be smaller since a number of these regions could be non-overlapping ESTs that would be merged into a single exon if the complete sequence was known. The majority of the novel found patterns are located in the Nterminal region (32 patterns) with additional seven occurring in the UCR1-UCR2 linker region, four mapping to the UCR1 region, six occurring in the UCR2 region, two occurring in the linker region between UCR2 and the catalytic core, and four occurring in the catalytic core itself. The locations of these previously undetected patterns is in line with previous research showing that the aminyl end of the protein is more susceptible to variability, producing long and short isoforms of PDE4 proteins $(157 ; 163)$. However, this study indicates additional exons in the carboxyl end, which was typically thought to be highly conserved due to the presence of a catalytic region. Molecular techniques verify that three of these exons are present with a degree of tissue specificity. 
A potential shortcoming of looking at ESTs for alternative splicing is that the 5' ends of the mRNAs are underrepresented, due to the fact that the majority of EST libraries are constructed using poly-T primers that anneal to the 3 ' end of the mRNAs. As a result, a number of exons in the variable region of the PDE4 family of genes are potentially missed. 


\section{CHAPTER V}

\section{COMPUTATIONAL METHOD FOR PSEUDOGENE DETECTION AND CHARACTERIZATION}

\subsection{Pseudogenes}

Genes are the basic unit to transfer hereditary information. They provide structure, function and regulation to a biological system. The genetic blueprint of an organism can be understood by studying the architecture of these genes. Pseudogenes, on the other hand, are regions sharing a level of sequence homology to functional genes that have somehow lost their ability to operate as genes. Pseudogenes may contain stop $\operatorname{codons}^{27}$, have frame shifts ${ }^{28}$ and a lack of transcription. However, they might retain gene-like features, such as promoters, $\mathrm{CpG}$ islands ${ }^{29}$ and splice sites, and are therefore of interest to biologists who wish to study the effects of evolutionary processes.

As discussed in Section 1.6, a gene must go through several steps from a genetic DNA sequence to a fully-functional protein: transcription, pre-mRNA processing, translation and protein folding (Figure 5.1). If any of these steps fails, the sequence may be considered nonfunctional. The most commonly identified disablements are stop codons and frame shifts, which almost universally stop the translation of a functional protein product.

\footnotetext{
${ }^{27}$ Stop codons are TAA, TAG and TGA.

${ }^{28}$ A frame shifting is a genetic mutation that inserts or deletes a number of nucleotides that is not evenly divisible by three from a DNA sequence.

${ }^{29} \mathrm{CpG}$ islands are short genomic regions that contain a high frequency of CG dinucleotides than in other regions. ' $\mathrm{p}$ ' indicates that ' $\mathrm{C}$ ' and ' $\mathrm{G}$ ' are connected by a phosphodiester bond.
} 


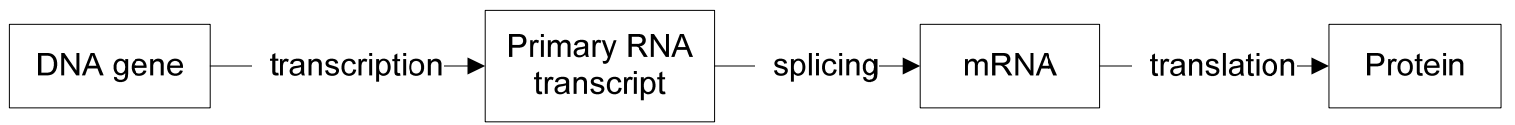

Figure 5.1: Central Dogma.

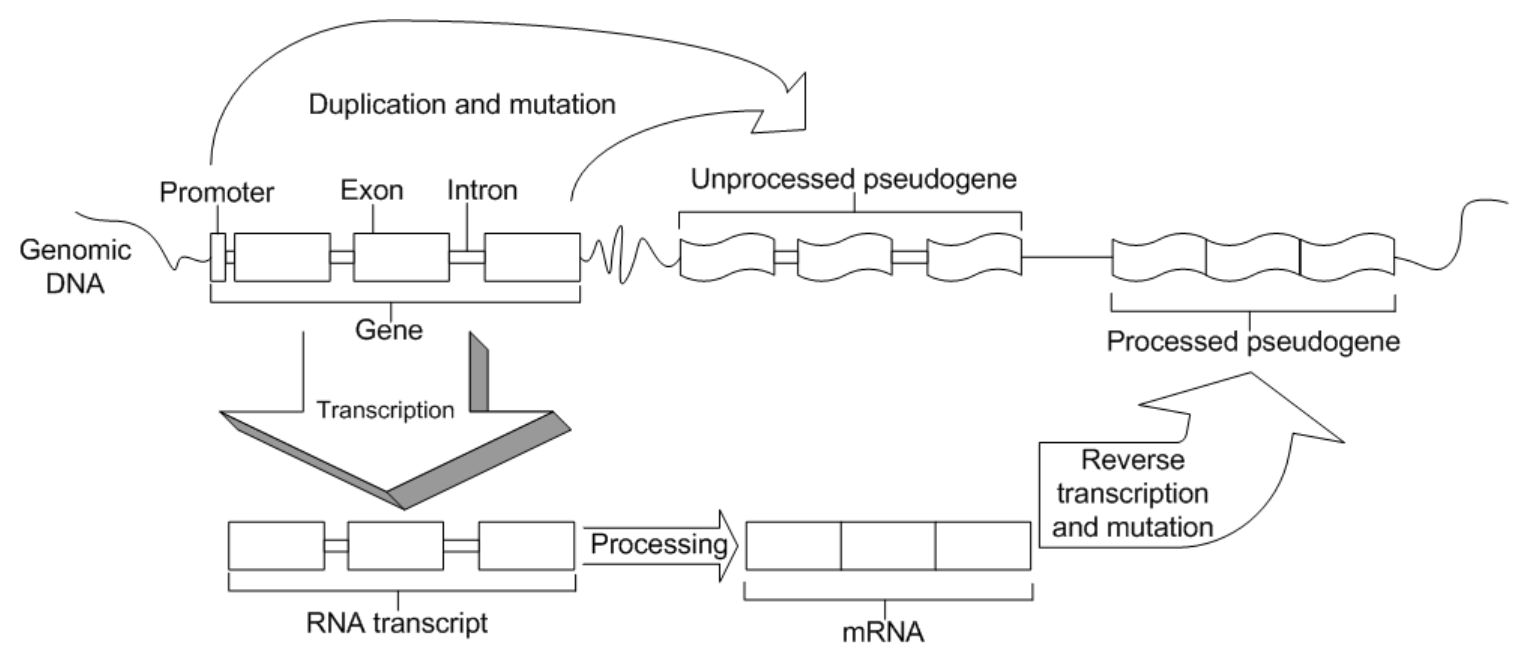

Figure 5.2: Pseudogene formation.

\subsubsection{Processed Pseudogenes}

Processed pseudogenes are generated by retrotransposition, which involves reverse transcription of an mRNA transcript and subsequent re-integration into genomic DNA. For human chromosome 22, $19 \%$ of the coding sequence corresponds to pseudogenes, $82 \%$ of which are processed pseudogenes (176;177). Processed pseudogenes represent the vast majority of inactivated gene sequences found in the human genome.

There are three basic features of a processed pseudogene. They are

1. A lack of non-coding intervening sequences (introns),

2. The presence of a poly-A tail at the 3' end, and 
3. Homological extension (flanking direct repeats, which are associated with movable genetic elements) $(176 ; 178)$.

In the human genome, there are a number of different estimations for the total number of processed pseudogenes. Zhang et al. predicts $\sim 7,800$ processed pseudogenes (179) while Torrents et al. estimates there are $\sim 13,800$ (180). Ohshima et al. report 3,664 processed pseudogenes in the human genome and estimate a total number $\sim 7,000$ processed pseudogenes, based on an estimation of $\sim 35,000$ human genes (181-183).

\subsubsection{Unprocessed Pseudogenes}

In the process of duplication, genetic sequences can go through various modifications, including mutation ${ }^{30}$, frame shifting, insertions and deletions. Any significant modification during the translational or transcriptional stage could result in a loss of genetic function. Hence, if the duplication of a gene is incomplete, the new sequence could be a pseudogene. Those pseudogenes derived from duplication are called unprocessed pseudogenes $(65 ; 176 ; 184)$. It arises when a cell is replicating its own DNA and inserts an extra copy of a gene into a genome in a new location (185).

Unprocessed pseudogenes may have introns retained (as shown in Figure 5.2). They can be a complete or partial copy from the parent gene. Sometimes, the unprocessed pseudogenes contain an extra copy of the gene. The unnecessary extra copy "could accumulate mutations without harming the organism" (178).

\footnotetext{
${ }^{30}$ A mutation is a change of the DNA sequence within a gene or chromosome of an organism resulting in the creation of a new character or trait not found in the parental type. Mutation can also mean the process by which such a change occurs in a chromosome, either through an alternation in the nucleotide sequence of the DNA coding for a gene or through a change in the physical arrangement of a chromosome.
} 
In addition, the unprocessed pseudogene can be created secondarily from preexisting processed pseudogenes. In other words, the parental source gene may be intronless. In this case, the duplication occurred after transcription (176).

\subsubsection{Disabled Pseudogenes}

A third type of pseudogenes, known as disabled or unitary pseudogenes, are becoming more widely studied $(186 ; 187)$. When various mutations occur, they can disrupt transcription or translation of a gene. Moreover, if these mutations become fixed in a population, it is possible the gene may become nonfunctional or deactivated in a mechanism similar to unprocessed pseudogenes. The difference between the two is that disabled pseudogenes are not duplicated before becoming disabled (188).

\subsubsection{Functional Pseudogenes}

Up to this point, pseudogenes have been defined as having no function in Sections 1.8.3 and 5.1.1-5.1.3. However, in 2003, Hirotsune et al. in Japan found that a retrotransposed pseudogene whose transcript plays a trans-regulatory role in the expression of its homologous gene, Makorin1. They suggested a general model that pseudogenes may play an important biological role $(189 ; 190)$. In 2004, Hirotsune and his team reported that about $2-3 \%$ of human processed pseudogenes are expressed using the strict criteria based on their algorithm and wet laboratory experimentation (191). However, their result has been strong contested by Gray's team due to the inability to reproduce Hirotsune's experiment (192). Therefore, the functional role of pseudogenes is of current interest. 


\subsubsection{Motivations for Pseudogene Detection and Characterization}

Computational sequence analysis can be complicated by the presence of pseudogenes. For instance, it is possible that a researcher wants to amplify a certain gene by using polymerase chain reaction (PCR) but accidently and simultaneously amplifies a pseudogene due to their share similar sequences. Another example is in $a b$ initio ${ }^{31}$ gene prediction where processed pseudogenes are often misidentified as real genes or exons. Identification of pseudogenes will help to improve the accuracy of gene prediction (183). In addition, identifying pseudogenes may lead to a better understanding of gene evolution.

\subsection{Current Automatic Pseudogene Detecting Methods}

\subsubsection{Harrison's Approach}

Harrison et al. from Yale University developed a pseudogene annotation pipeline in 2001 (193). They used Caenorhabditis elegans genome to test their seven-step process. First, they downloaded annotated pseudogene sequences from Sanger Centre and analyzed those sequences. They found about $6 \%$ of the annotated pseudogenes are not detectable when considering simple disablement, such as a frameshift or premature stop codon. Second, they aligned protein sequences (Wormpep18, SCOP and PROTOMAP) to the genome sequences using FASTA in order to find potential pseudogenes. All sixframe sequences (three forward and three backward strands) were analyzed. Third, alignment results were filtered for overlapping matches in genomic sequences, if the size

\footnotetext{
${ }^{31}$ In computation, $a b$ initio means an extra, transform, load tool used to manipulate data. In Bioinformatics, $a b$ initio is a term used to define methods for making predictions about biological features using only a computational model without extrinsic comparison to existing data. In this context, it may be sometimes interchangeable with the Latin term de novo.
} 
of the overlap is over 30 nucleotides. Fourth, to avoid over-counting for worm protein hits, the initial matches were further filtered for adjacent matches. Fifth, the potential pseudogenes were filtered for overlap with any other annotations in the Sanger Centre files such as exons of genes, tandem or inverted repetitive and transposable elements. Sixth, they refined the data for additional repeat elements and any match to proteins. Seventh, they increased the threshold e-value from 0.01 to 0.001 (193).

In 2002, using the same methodology, Harrison et al. released their results for detecting pseudogenes in human chromosome 21 and 22. They found 77 processed pseudogenes on chromosome 21 and 112 on chromosome 22 (194). For unprocessed pseudogenes, they found 72 on chromosome 21 and 123 on chromosome 22 (194).

\subsubsection{Sakai’s Approach}

In 2003, Sakai et al. from Japan introduced a method to detect processed pseudogenes based on cDNA mapping to the human genome (195). Their methods were divided into two sections. The first section is the cDNA mapping. They aligned all cDNA sequences against human genome using blastn. Then they extracted corresponding genomic regions for each query sequence. Next, they set up cut values of $\geq 95 \%$ identity and $\geq 80 \%$ coverage to filter those sequence hits based on the est2genome.

The second stage of the experiment is pseudogene detection. All the candidate sequences collected from the first stage were classified into six classes according to the way in which the introns are processed (Figure 5.3) before checking the 3' terminator. 


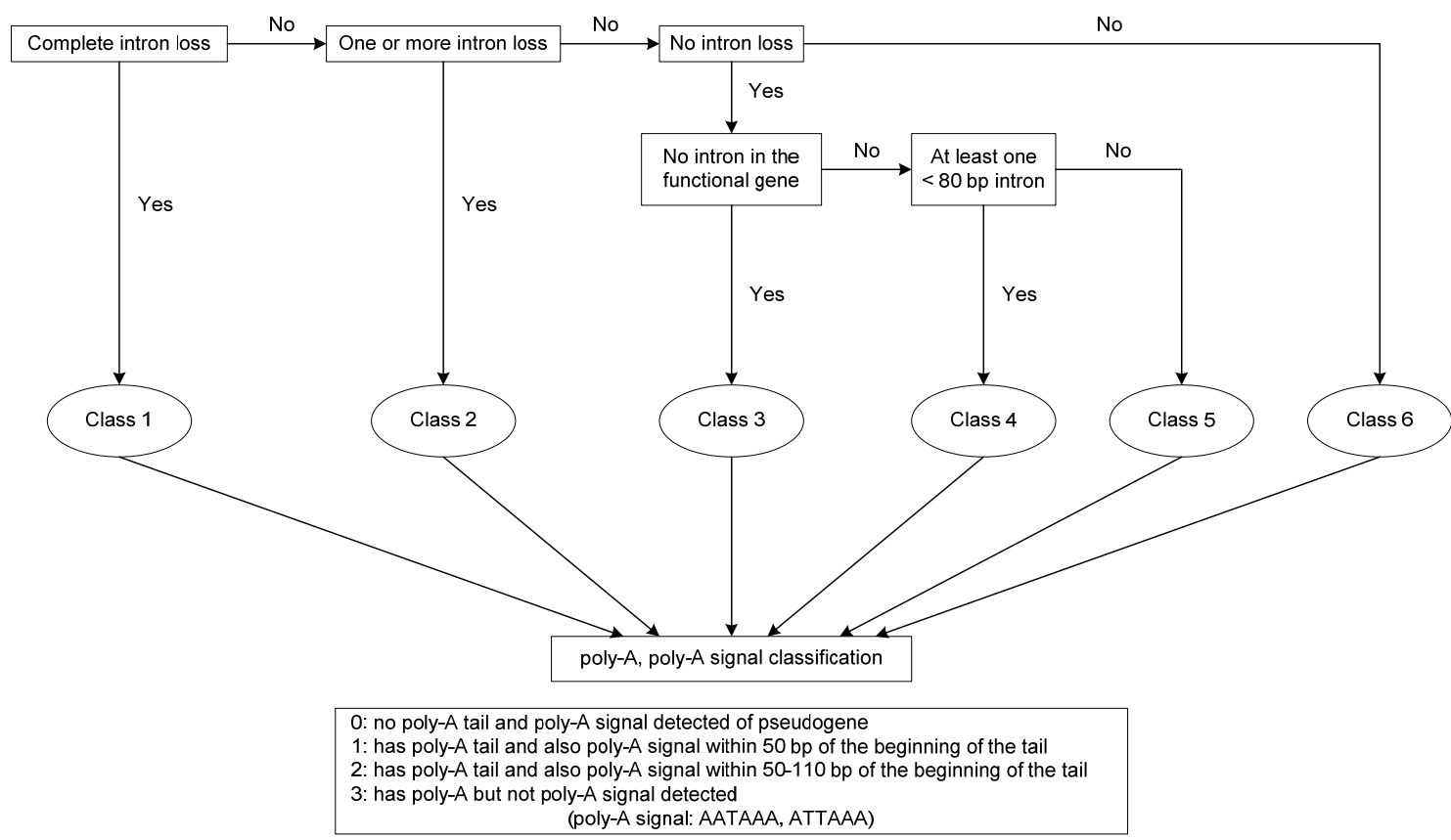

Figure 5.3: Flow chart of Sakai et al. method for processed pseudogene detection.

Sakai and his team found 6,549 possible processed pseudogenes using 113,196 human cDNA with their automated pipeline including novel intron-containing pseudogenes. They believed their approach will help aid the accuracy of gene prediction (195).

\subsubsection{PPFINDER}

PPFINDER was released in 2006 to find processed pseudogenes (183). The purpose for the program is to improve the accuracy of gene prediction methods, if processed pseudogenes can be identified. A gene model was first established in order to collect the gene information. Two methods were used in order to find pseudogenes: intron location and conserved synteny. In the intron location method, it started to align pre-established gene model to cDNA database using blastn. The best scoring hits were 
kept. Then, these transcripts were further aligned to genome locus. If the transcript hits more than one location in the genome, the transcript will be marked as pseudogenederived for further testing. The segments of predicted gene will only hit themselves in the genome, if they are not pseudogene-derived. But if they are pseudogene-derived, the best hit score will be with the parental gene location. The other hit locations in the genome can be potential pseudogenes.

In the synteny method, the candidate pseudogene-derived sequences were split into exons and translated into amino acids. Then these translated sequences were aligned to the protein database using blastp. If the sequence identity of the hit is $\geq 65 \%$ and the length of the hit is at least 10 amino acids, these sequences were kept. Then the sequences were examined if their hit location is the same as the gene model. If the answer is no, the information of conserved syntenic region will be recorded. Once again, they aligned the original created gene model to the recorded information to find orthologous region. If the orthologous regions were found, the gene model is said to contain a pseudogene.

However, if it is a single-exon gene, without locating introns, the sequence might be counted as a repeat and would be failed to identify as pseudogene-derived sequence (183).

\subsubsection{PseudoPipe}

Zhang et al. from Yale University proposed an automated pseudogene identification pipeline in 2006 (196). Their method was based on Harrison's approach (both Harrison and Zhang were post-docs training in Gerstein's laboratory at Yale University). This pipeline began by aligning protein sequences to genomic sequences 
using tblastn. Then the sequences overlapping with the gene sequences in ENSEMBL database were removed. Smith-Waterman was used to optimize the alignment to merge and extend candidate sequences. Fourth, false positive, repeats, low complexity sequences and potential functional gene candidates were removed. The pseudogene candidate is considered a false positive if its BLAST e-value is $\leq 10^{-10}$ or an amino acid sequences identity is $\leq 40 \%$. Finally, sequences are separated into different classes retrotransposed pseudogenes, duplicated pseudogenes (or unprocessed pseudogenes in our definition), or pseudogeneic fragments based upon exon structure $(179 ; 182 ; 196 ; 197)$.

\subsubsection{Pseudogenes Analysis in ENCODE Project}

In Section 1.7.4, the ENCODE project was discussed. It brought dozen of laboratories together to participate to analyze and identify the functional elements in the human genome. Studies were focused on transcripts and gene models, protein-binding sites, epigenetic markers, and promoters and enhancers, evaluating the existing methods and creating new methods if necessary. One group focused on methods for detecting pseudogenes.

As a subgroup of ENCODE, the pseudogene research team focused on establishing methods to decide consensus annotation, analyze transcription and study evolution. The first goal was to "obtain an accurate list of pseudogenes in order to facilitate the creation of a comprehensive catalog of structural and functional elements in the ENCODE regions" (47;56). They evaluated five developed methods: GIS-PET (198), HAVANA (199), PseudoPipe (55;196), pseudoFinder (unpublished), and retroFinder $(200 ; 201)$. 
The pseudogene research team found that all five methods detect pseudogenes based on sequence similarity to "at least one entry in a collection of query sequences representing known human genes (referred to as the parent genes)." The main two differences among these five methods are in (1) type of queries, either nucleotides or proteins, used to search for pseudogenes and (2) strategies used to assess a sequence's coding potential and methods for distinguishing processed from unprocessed pseudogenes (56).

The second task was to establish a consensus procedure including manual curation in order to get the correct and reliable list of pseudogenes. The research team only considered those pseudogenes that match known proteins from the UniProt database (202).

After evaluating the five computational methods, they characterized the sequences they found and sequenced these pseudogene transcripts. They realized that in the ENCODE gene-rich regions, more pseudogenes were found. In addition, most sequences are decayed gene copies that contain frame shift mutations (56).

\subsection{Characteristics for Pseudogene Detection}

In the previous sections, different types of pseudogenes and the current computational methods have been discussed. Based on the biological factors and other's work, there are some features that need to be investigated when evaluating pseudogene candidate sequences. They are start and stop codons, poly-A signals and poly-A tails, $\mathrm{G}+\mathrm{C}$ composition, and mutational events. Each of these features is considered in a newly developed method to score sequences for their likelihood of being a pseudogene. The rest 
of Section 5.3 covers in detail the features considered for the method constructed in Section 5.5.

\subsubsection{Start Codon}

When a fully-functional protein is translated from a genetic sequence, it goes through transcription, pre-mRNA processing, translation and protein folding (Figure 5.1). By default, ATG, or AUG in RNA sequences, serves as a start codon or initiator to translate genes to proteins (203). There are rare cases in eukaryotes initiated by codons other than ATG/AUG (204-209), such as GTG/GUG. The standard code currently allows initiation from TTG/UUG and CTG/CUG in addition to ATG/AUG (203).

When detecting pseudogenes, locating a conserved or mutated ATG in an open reading frame is a good starting point. It is possible that ATG will be located in a different or a later place other than the first position in a sequence. The alternative patterns of ATG from Table 5.1 are examined. 
Table 5.1: Start Codon Alternative Patterns and Their Scores

\begin{tabular}{l|lllll}
\hline Main pattern & \multicolumn{5}{|c}{ Alternative patterns } \\
\hline \multirow{4}{*}{ ATG 1.00 } & ATN & ATA & ATC & ATT & 0.25 \\
\cline { 2 - 6 } & ANG & AAG & ACG & AGG & 0.25 \\
\cline { 2 - 6 } & & AAN & ACN & AGN & \\
& ANN & AAA & ACA & AGA & 0.0625 \\
& & AAC & ACC & AGC & \\
& & AAT & ACT & AGT & \\
\cline { 2 - 6 } & NTG & CTG & GTG & TTG & 0.25 \\
\cline { 2 - 6 } & & CNG & GNG & TNG & \\
& NNG & CAG & GAG & & 0.0625 \\
& & CCG & GCG & TCG & \\
& & CGG & GGG & TGG & \\
\cline { 2 - 6 } & & CTN & GTN & TTN & \\
& NTN & CTA & GTA & TTA & 0.0625 \\
& CTC & GTC & TTC & \\
& & CCT & GGT & TTT & \\
\cline { 2 - 6 } & NNN & & & & 0.015625 \\
\hline
\end{tabular}

\subsubsection{Stop Codons}

A terminator is present to tell the cell to end the translation process and release the amino acid chain. TGA, TAG and TAA (or UGA, UAG, and UAA in RNAs) are three well-known stop codons (210-212). Stop codon insertion and mutation is one of the common features differentiating pseudogenes from regular genes. Pseudogenes are caused by either retrotranscription or duplication. Frame shift, mutation, insertion or deletions may cause the terminating signal relocate earlier in the sequences. In such a case, in the open reading frame, the premature stop codon causes translation to terminate. Finding premature stop codons is the most common feature to consider a sequence being a pseudogene sequence. Two conditions are considered:

- Presence of a start codon near the beginning of a sequence 
- Presence of a stop codon located downstream the start codon

Besides the most widely found stop codons, TGA/UGA, TAG/UAG, and TAA/UAA, an additional thirteen mutational patterns were inspected as well (Table 5.2).

Table 5.2: Stop Codon Alternative Patterns and Their Scores

\begin{tabular}{|c|c|c|c|c|c|c|}
\hline \multicolumn{2}{|c|}{ Main pattern } & \multicolumn{5}{|c|}{ Alternative patterns } \\
\hline \multirow{2}{*}{$\begin{array}{l}\text { TGA } \\
\text { TAG }\end{array}$} & \multirow{2}{*}{$\begin{array}{l}1.00 \\
1.00\end{array}$} & TGN & TGC & TGG & TGT & 0.25 \\
\hline & & TNG & TCG & & TTG & 0.25 \\
\hline \multirow[t]{22}{*}{ TAA } & \multirow[t]{22}{*}{1.00} & TAN & TAC & & TAT & 0.50 \\
\hline & & TNA & TCA & & TTA & 0.50 \\
\hline & & & TCN & & TTN & \\
\hline & & TNN & TCC & & TTC & 0.25 \\
\hline & & & TCT & & TTT & \\
\hline & & NAA & AAA & CAA & GAA & 0.25 \\
\hline & & NAG & AAG & CAG & GAG & 0.25 \\
\hline & & NGA & AGA & CGA & GGA & 0.25 \\
\hline & & & ANA & CNA & GNA & \\
\hline & & NNA & ACA & CCA & GCA & 0.0625 \\
\hline & & & ATA & CTA & GTA & \\
\hline & & & ANG & CNG & GNG & \\
\hline & & $N N G$ & ACG & CCG & GCG & 00625 \\
\hline & & Tive & AGG & CGG & GGG & \\
\hline & & & & CTG & GTG & \\
\hline & & & AAN & CAN & GAN & \\
\hline & & NAN & AAC & CAC & GAC & 0.0625 \\
\hline & & & AAT & CAT & GAT & \\
\hline & & & AGN & CGN & GGN & \\
\hline & & NGN & AGC & CGC & GGC & 0.0625 \\
\hline & & & AGT & CGT & GGT & \\
\hline & & NNN & & & & .015625 \\
\hline
\end{tabular}

\subsubsection{Poly-A Signals and Poly-A Tails}

Polyadenylation is the covalent linkage of a poly-A tail to an mRNA molecule in order to stabilize the molecule so a mature mRNA will be ready for translation and produce an amino acid. The process starts by finding a cleavage site which is 
characterized by the presence of a poly-A signal sequence near the cleavage site, mostly commonly found to be AATAAA (or AAUAAA in RNA). When the mRNA has been cleaved, a number of adenine (A) residues (50-250) will be attached in the 3 ' end at the cleavage site. The reaction is catalyzed by polyadenylate polymerase (213).

When pseudogenes are formed through retrotranscription or duplication, the incompletely translated sequences may be re-inserted into the genomic sequences. Those sequences may contain a poly-A site, just like processed mRNAs. It is also possible they have a number of adenines (A) in the 3 ' end of the sequence. In fact, most processed pseudogenes have shown the presence of a poly-A tail in the 3' end.

Table 5.3: Poly-A Signal Patterns

\begin{tabular}{crcr}
\hline polyA site & $\%$ sites & polyA site & $\%$ sites \\
\hline AATAAA & 58.2 & AATAGA & 0.7 \\
ATTAAA & 14.9 & AAAAAG & 0.8 \\
AGTAAA & 2.7 & ACTAAA & 0.6 \\
TATAAA & 3.2 & AAGAAA & 1.1 \\
CATAAA & 1.3 & AATGAA & 0.8 \\
GATAAA & 1.3 & TTTAAA & 1.2 \\
AATATA & 1.7 & AAAACA & 0.5 \\
AATACA & 1.2 & GGGGCT & 0.3 \\
\hline Abbreviations: poly-A, polyadenine; \\
\%, percentage. These 16 poly-A sites are collected \\
from Table 2 in (214).
\end{tabular}

AATAAA is the most common poly-A signal in human genome, found in close to $60 \%$ of gene sequences. ATTAAA is also relatively common, occurring $14.9 \%$ of the time. There are fourteen other patterns been identified as poly-A signals (Table 5.3). Based on their occurrence rate, different scores were assigned for each poly-A signal patterns. 
For poly-A tails, finding a large number of adenine (A) residues in a sequence is too difficult since these regions can also be counted as low complexity. Instead, we detect regions with a run of at least ten adenines, increasing the score according to the total number of A's found. If a candidate sequence owns a poly-A signal and a poly-A tail of length ten or more, the possibility being a pseudogene is greatly increased.

\subsubsection{G+C Composition}

In Harrison et al.'s research, they found the pseudogenes have higher $\mathrm{G}+\mathrm{C}$ composition than the background genomic DNA in general $(215 ; 216)$. The $\mathrm{G}+\mathrm{C}$ composition ranges from 36 to 54 percent in the sequences (217). Therefore, the candidate sequences will be reviewed to see how many G's and C's are involved in the sequences.

\subsubsection{Similar, Synonymous vs. Nonsynonymous Mutations}

When two sequences are counted as homologous, they share a certain percentage of similarity. Both of them have originated from a common ancestral sequence but accumulated various mutations. Figure 5.4 shows the types of mutations occurring an example pair of sequences. Although there are three differences between these two sequences, a single substitution, sequential substitution, coincidental substitution, parallel substitution, convergent substitution and back substitution have also occurred.

In Section 1.4, the genetic code was discussed. The $4 \times 4 \times 4$ or 64 different combinations of codons from $\mathrm{A}, \mathrm{C}, \mathrm{G}$ and $\mathrm{T}$ represent the twenty common amino acids. Based on Table 5.4, we can translate these two descendant sequences into amino acids. 
Although there are only three nucleotides changed in each descendant sequence, some of the amino acids have been changed.

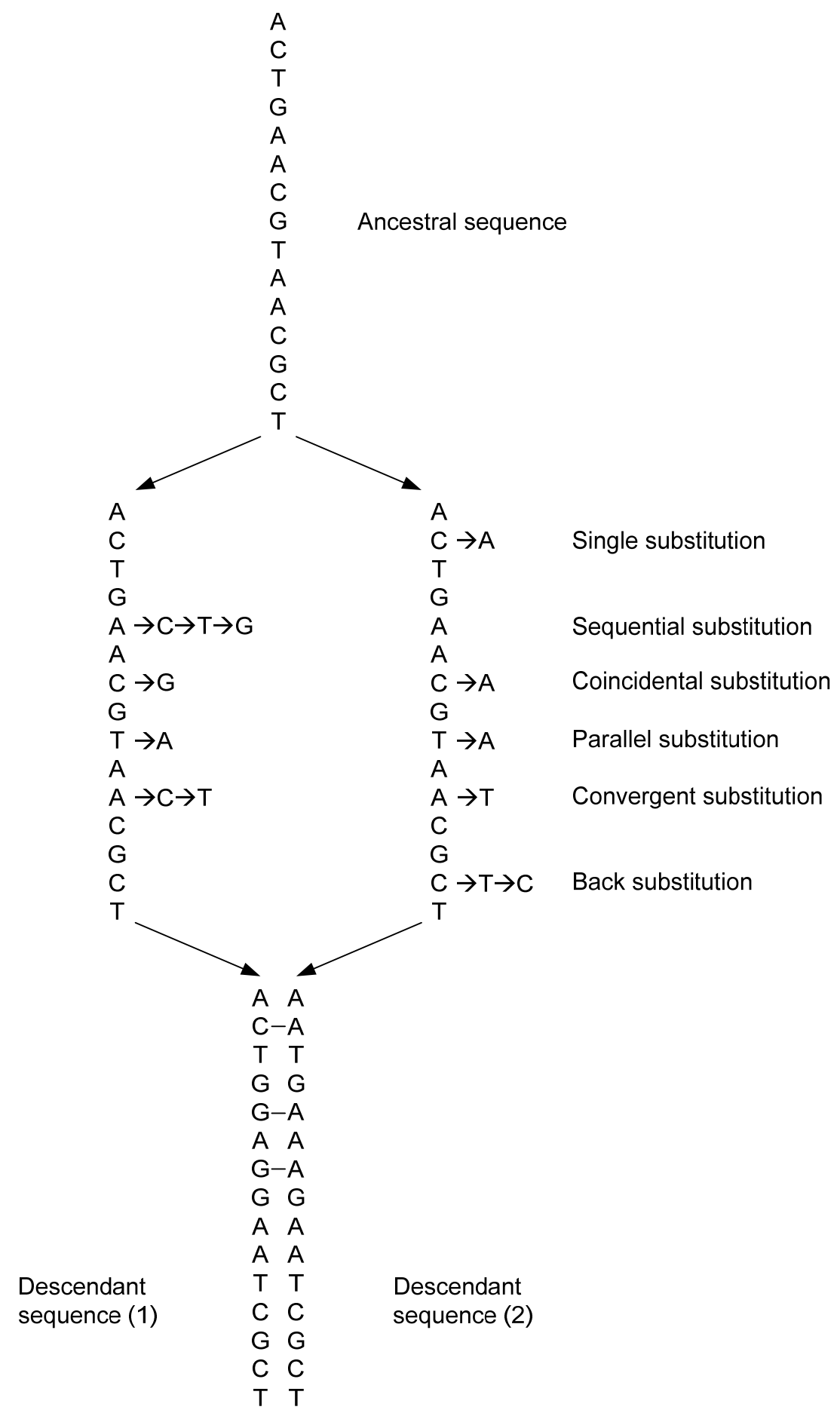

Figure 5.4: Mutations occurring in two homologous sequences. Image adopted and modified from Figure 3.6 in (218). 
Table 5.4: The Genetic Code (RNA to Amino Acids)

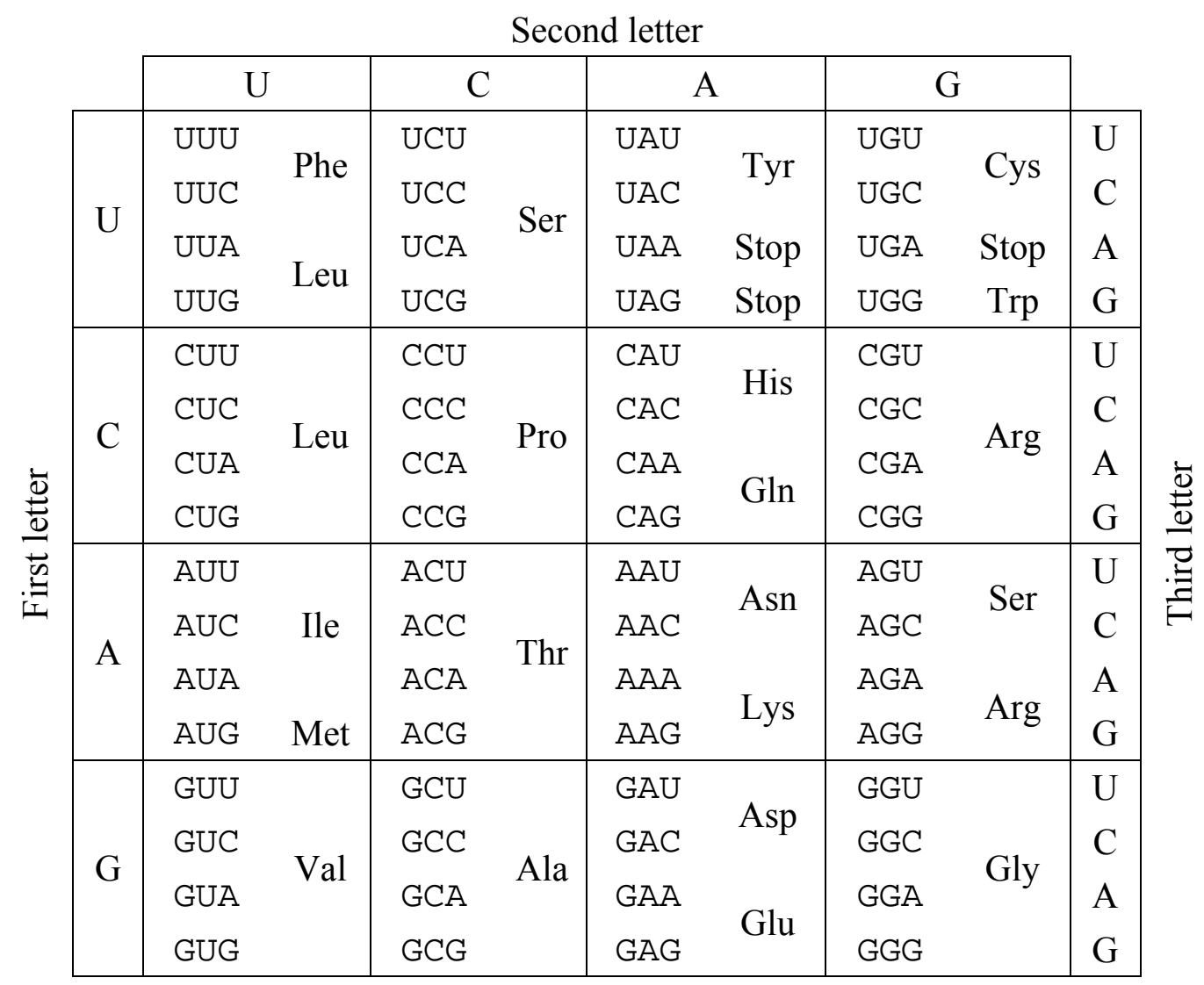

In descendant sequence (1), the $5^{\text {th }}, 7^{\text {th }}, 9^{\text {th }}$ and $11^{\text {th }}$ bases have been changed after mutations. Although it does not look like a lot of changes in the nucleotide acid sequence, the amino acids which contain these four mutational nucleotides respectively have been changed (Figure 5.5).

\begin{tabular}{|c|c|c|c|c|c|c|c|c|c|c|c|}
\hline Thr & \multicolumn{2}{|c|}{ Glu } & \multicolumn{3}{|c|}{ Arg } & \multicolumn{3}{|c|}{ Asn } & \multicolumn{3}{|c|}{ Ala } \\
\hline $\begin{array}{lll}A & C & T\end{array}$ & & $\begin{array}{l}A \\
A \\
\uparrow\end{array}$ & c & & & A & $\begin{array}{l}A \\
\uparrow\end{array}$ & C & G & C & $\mathrm{T}$ \\
\hline $\begin{array}{c}\text { A } \underset{T h r}{C} \\
T\end{array}$ & & $\begin{array}{l}\text { G A } \\
\text { Gly }\end{array}$ & & $\begin{array}{c}\text { G } \\
\text { Gly }\end{array}$ & A & $A$ & $\begin{array}{l}\text { T } \\
\text { Ile }\end{array}$ & C & & $\begin{array}{c}\text { C } \\
\text { Ala }\end{array}$ & $\mathrm{T}$ \\
\hline 123 & 4 & 56 & 7 & 8 & 9 & 10 & 11 & 12 & 13 & 14 & 15 \\
\hline
\end{tabular}

Figure 5.5: Comparison between ancestral sequence and descendant sequence (1). 
In descendant sequence (2), there are also three mutations locating in the $2^{\text {nd }}, 7^{\text {th }}$, $9^{\text {th }}$ and $11^{\text {th }}$ bases. Two of the three amino acids which contain the three mutational nucleotides respectively have been changed (Figure 5.6).

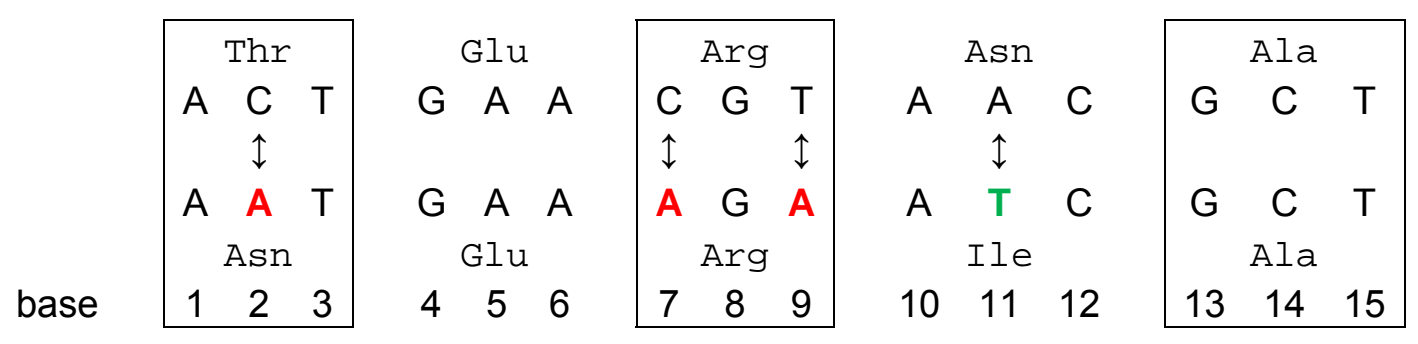

Figure 5.6: Comparison between ancestral sequence and descendant sequence (2).

The $7^{\text {th }}$ and $9^{\text {th }}$ nucleotides changed in descendant sequence (2) can be counted as synonymous mutations because the amino acid does not change. However, the other mutational nucleotides shown in Figures 5.5 and 5.6 that change the amino acids have to be studied further in order to distinguish if they produce nonsynonymous or similar synonymous mutation.

Those mutations at the nucleic level that result in a similar but not identical amino acid are called similar synonymous mutations. Although the mutational nucleotide does change the amino acid, the functionality of the amino acid is close to the original one. But if mutations at the nucleic level change the amino acid to something unrelated, it is called a nonsynonymous mutation. To find out how similar or how close the amino acids are between each other, the PAM250 matrix generated by Dayhoff (97) and the BLOSUM62 matrix generated by Henikoff $(219 ; 220)$ are the two matrices widely used. In WU BLAST, BLOSUM62 matrix is chosen by default when comparing protein sequences. If 
the comparison results a same amino acid, it will display the amino acid. But if the comparison returns a close amino acid, it will give a plus '+' sign.

\begin{tabular}{|c|c|c|c|c|c|c|c|c|c|c|c|c|c|c|c|c|c|c|c|c|c|}
\hline Subject & G & $\begin{array}{l}A \\
E\end{array}$ & G & A & $\begin{array}{l}A \\
N\end{array}$ & $\mathrm{~T}$ & C & $\begin{array}{l}C \\
P\end{array}$ & C & $A$ & $\begin{array}{l}A \\
K\end{array}$ & G & A & $\begin{array}{l}\mathrm{A} \\
\mathrm{K}\end{array}$ & G & $\mathrm{T}$ & $\begin{array}{l}A \\
Y\end{array}$ & C & A & $\begin{array}{l}\mathrm{T} \\
\mathrm{I}\end{array}$ & C \\
\hline blastx & & $E$ & & & & & & $P$ & & & K & & & K & & & + & & & I & \\
\hline Query & G & $\begin{array}{l}A \\
E\end{array}$ & G & $A$ & $\begin{array}{l}\mathrm{C} \\
\mathrm{T}\end{array}$ & $\mathrm{T}$ & C & $\begin{array}{l}C \\
P\end{array}$ & C & A & & G & A & $\begin{array}{l}\mathrm{A} \\
\mathrm{K}\end{array}$ & G & C & $\begin{array}{l}\mathrm{A} \\
\mathrm{H}\end{array}$ & C & A & $\begin{array}{l}\mathrm{T} \\
\mathrm{I}\end{array}$ & C \\
\hline base & 1 & 2 & 3 & 4 & 5 & 6 & 7 & 8 & 9 & 10 & 11 & 12 & 13 & 14 & 15 & 16 & 17 & 18 & 19 & 20 & 21 \\
\hline
\end{tabular}

\section{Figure 5.7: Similar synonymous mutation vs. nonsynonymous mutation.}

Subject, subject sequences (nucleotide and amino acid); blastx, results returned by blastx; Query, query sequences (nucleotide and amino acid).

In Figure 5.7, the nucleotides located at the $5^{\text {th }}$ and $16^{\text {th }}$ bases have mutated. Both amino acids which contain these two mutational nucleotides are changed as well. According to the blast $x$ result, the amino acid changed from $N$ (Asn) to $T$ (Thr) is not related (blank in the blastx result row). The nucleotide substitution is a nonsynonymous mutation. But the amino acid changed from $\mathrm{Y}$ (Tyr) to $\mathrm{H}$ (His) is related (plus ' + ' sign in the blastx result row). Thus, the nucleotide substitution is called a similar synonymous mutation.

To evaluate the pseudogene candidates, determining the number of similar synonymous mutations (the amino acid is changed to a close amino acid), synonymous mutations (the amino acid is not changed) and nonsynonymous mutations (the amino acid is changed to a unrelated amino acid) will be calculated. 


\subsection{Sequence Data}

All the approaches discussed in Section 5.2 begin pseudogene analysis by aligning sequences to genomic regions. Besides Sakai et al. who use cDNA sequences, all other approaches use protein sequences or RefSeq sequences for alignments.

Similar to the methods that have been discussed in Section 5.2, the pseudogene detection and characterization in this project begins with sequence alignment. Rather than using cDNA, RefSeq or protein sequences in as other approaches, EST sequences were chosen in this project. Compared to cDNA, RefSeq or protein sequences, ESTs are relatively short, ranging from 300 to $600 \mathrm{bp}$ in length. The size of cDNA, RefSeq or protein sequences could range from 1000 to $4000 \mathrm{bp}$. Aligning ESTs to genomic sequences will return more hits from different locations of genomic regions due to their smaller size. It is possible to return the high scoring pairs (HSP) from the genomic locations that are never reported before from the previous methods due to longer query sequences.

Although it will increase the amount of data for further analysis, it is possible to provide more candidate locations in genomic regions as pseudogenes. Moreover, with the choices of alternative splicing (which has been studied in Chapter 4), it will bring an additional possibility to locate genomic hits other than the parental hit itself.

In the current approaches, EST sequences have not been used for identifying pseudogenes. Hence, using ESTs for initial alignment for searching and determining candidate pseudogenes provides a novel source of data. 


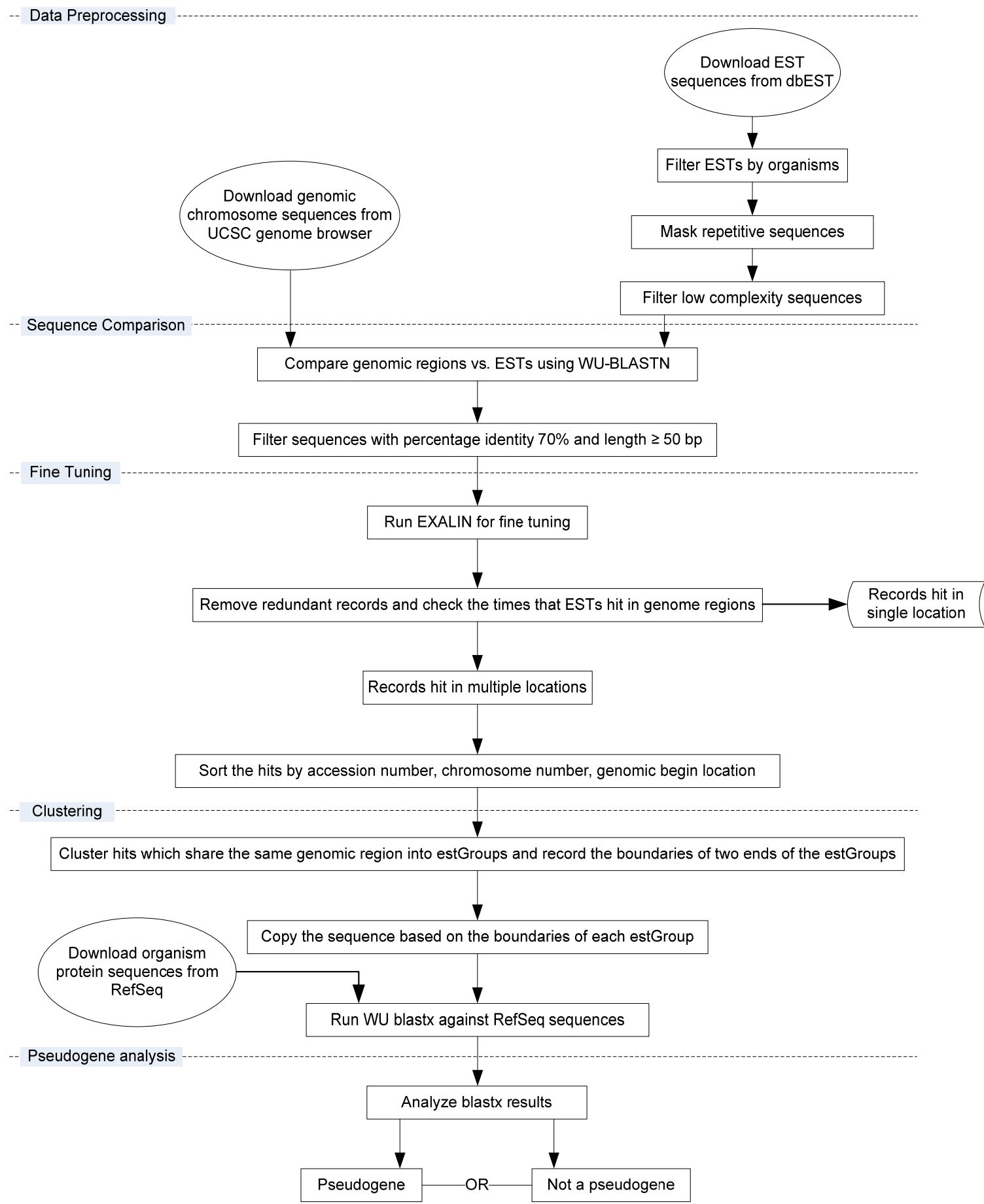

Figure 5.8: Process for pseudogene detection. 
Pseudogenes can be detected in the steps out-lined in Figure 5.8. After downloading the necessary sequence files, each sequence will go through data preprocessing, sequences alignment, clustering and pseudogene detection. The detailed information for the methodology will be discussed in Section 5.6.

Expressed sequence tags (ESTs) were downloaded from GenBank/dbEST release 158.0, dated 4-18-2007 (http://www.ncbi.nlm.nih.gov/dbEST/) (72) for the purpose of detecting and characterizing pseudogenes. RefSeq sequences were downloaded from NCBI/RefSeq release 22, dated 3-13-2007 (http://www.ncbi.nlm.nih.gov/RefSeq/) (7376). Genomic regions were downloaded from UCSC Genome Browser (http://genome.ucsc.edu) (77-81).

\subsection{Methodology}

\subsubsection{Data Preprocessing}

EST sequences downloaded from GenBank/dbEST are parsed to include only those from the species of interest. Raw EST sequences are masked for repetitive elements using RepeatMasker $(87 ; 88)$. Low complexity regions for ESTs are then masked using the DUST (70) filter available from the wuBLAST software $(70 ; 167)$.

\subsubsection{ESTs to Genome Alignment}

After organism sequences are filtered, ESTs are aligned to genomic regions using wublastn with parameters $\mathrm{W}=15$ topComboN $=5-$ nogaps - gspmax $=100-$ hspmax $=500$ filter $=$ dust - warning - notes - error - links. The results run by blastn are then filtered by a Perl script to parse out the necessary information (Figure 5.9). 


\begin{tabular}{|c|c|c|c|c|c|c|c|c|c|c|}
\hline \multicolumn{11}{|c|}{$\begin{array}{l}=g i|5555714| g b|A V 175813.1| / g \\
\text { cDNA: Strain N2 hermaphrodit } \\
3^{\prime}(300 \text { letters; record } 79)\end{array}$} \\
\hline$>$ chrIII & 741 & 5971574 & 5971723 & 33 & 182 & 1 & (1) -2 & 0.99 & 0.50 & 0.50 \\
\hline$>$ chrIII & 593 & 5972503 & 5972622 & 181 & 300 & 1 & $1-(2)$ & $\odot .99$ & 0.40 & 0.40 \\
\hline >chrIII & 136 & 12875675 & 12875730 & 36 & 91 & 2 & (3) -4 & 0.71 & 0.19 & 0.13 \\
\hline >chrIII & 95 & 13163938 & 13163974 & 84 & 120 & 2 & $3-(4)$ & 0.73 & 0.12 & 0.09 \\
\hline >chrIII & 124 & 13114293 & 13114342 & 58 & 107 & 3 & (5) & 0.72 & 0.17 & 0.12 \\
\hline >chrII & 123 & 12241150 & 12241215 & 46 & 111 & 1 & $(1)$ & 0.65 & 0.22 & 0.14 \\
\hline >chrII & 120 & 15033827 & 15033868 & 69 & 110 & 2 & $3-(2)$ & 0.76 & 0.14 & 0.11 \\
\hline >chrII & 97 & 1139631 & 1139653 & 44 & 66 & 2 & (3) -2 & 0.91 & $\odot .08$ & 0.07 \\
\hline >chrV & 130 & 19475058 & 19475101 & 76 & 119 & 1 & $2-(1)-3$ & 0.77 & 0.15 & 0.11 \\
\hline >chrV & 103 & 8552104 & 8552129 & 39 & 64 & 1 & (2) $-1-3$ & 0.88 & 0.09 & 0.08 \\
\hline >chrV & 94 & 20038590 & 20038642 & 173 & 225 & 1 & $2-1-(3)$ & 0.64 & 0.18 & 0.11 \\
\hline >chrIV & 128 & 11169253 & 11169310 & 88 & 145 & 1 & $2-(1)-3$ & 0.69 & 0.19 & 0.13 \\
\hline >chrIV & 103 & 7401906 & 7401958 & 42 & 94 & 1 & (2) $-1-3$ & 0.66 & 0.18 & 0.12 \\
\hline >chrIV & 93 & 12955563 & 12955595 & 195 & 227 & 1 & $2-1-(3)$ & 0.76 & 0.11 & $\odot . \odot 8$ \\
\hline >chrIV & 124 & 1160745 & 1160812 & 45 & 112 & 2 & $(4)-5$ & 0.65 & 0.23 & 0.15 \\
\hline >chrIV & 102 & 9483397 & 9483483 & 97 & 183 & 2 & $4-(5)$ & 0.57 & 0.29 & 0.17 \\
\hline >chrIV & 124 & 959701 & 959768 & 37 & 104 & 3 & $(6)$ & 0.65 & 0.23 & 0.15 \\
\hline
\end{tabular}

\section{Figure 5.9: Sample blastn result for GI 5555714.}

Column 1, chromosome; column 2, high scoring pair score; columns 3 and 4, begin and end locations in genomic regions; columns 5 and 6, begin and end locations in ESTs; column 7, group; column 8, links; columns 9,10 and 11, percent identity, percent coverage and match value.

Each record starts with an equal sign '=' followed by a description of the EST.

The following rows, starting a greater sign ' $>$ ' represents a sequence hit, including the chromosome number, HSP score, begin and end locations of the hits in the genomic sequences, begin and end locations of the hit in EST, the group number, the links, the percent identity of the hit, the percent coverage of the hit and the match value of the hit.

Since pseudogenes have a high similarity to the genome sequence of origin and ESTs have a 2-5\% sequence error rate (221), only sequences whose identified similarities are greater than or equal to $95 \%$ are reserved. Ideally, these sequences are closer to the target pseudogenes. However, the hit size has to be considered as well. If the HSP is greater or equal to $95 \%$ but hits fewer than 40 bases out of a 400 bp EST, the coverage is low. 
The blastn computational results were filtered based on the following criteria: if it is a single splice hit, discard hits with length less than $50 \mathrm{bp}$. If it is multiple-splice hit, the sequence hit has exactly two splices, and the gap between two hits are over $1,000,000 \mathrm{bp}$, discard the record. Otherwise, measure the size of the hit with each splice and discard the record whose percent identity is less than $70 \%$. Results are compared (single-splice hits and multiple-splice hits) and sorted by: accession number, chromosome number and genomic begin location. A flowchart of this process is provided in Figure 5.10.

As discussed in Sections 1.8 and 4.1.3 to 4.1.5, a gene sequence is derived from the combination of exons after the introns are removed. Those highly similar sequences which have been filtered might be the splices of genes themselves. Therefore, additional sequence comparison is needed. The set of spliced nucleotide sequences will be aligned to an unspliced genomic DNA sequence. Through this process, beginning and ending locations of exons and introns will be discovered.

In addition, GT/AG and CT/AG are commonly found at exon/intron boundaries known as donor and acceptor sites. Once the beginning and ending locations of exons and introns are known, the splice patterns of the gene can be detected. Most introns begin with GU (or GT in DNA) and end with AG patterns. With this information, the exact boundary between any two exons can be found by mapping ESTs back to the genome. However, the frequency of GU-AG combinations of bases is fairly common, occurring on an average about $1 / 16$ of the time. A number of programs help distinguish these combinations (222). 


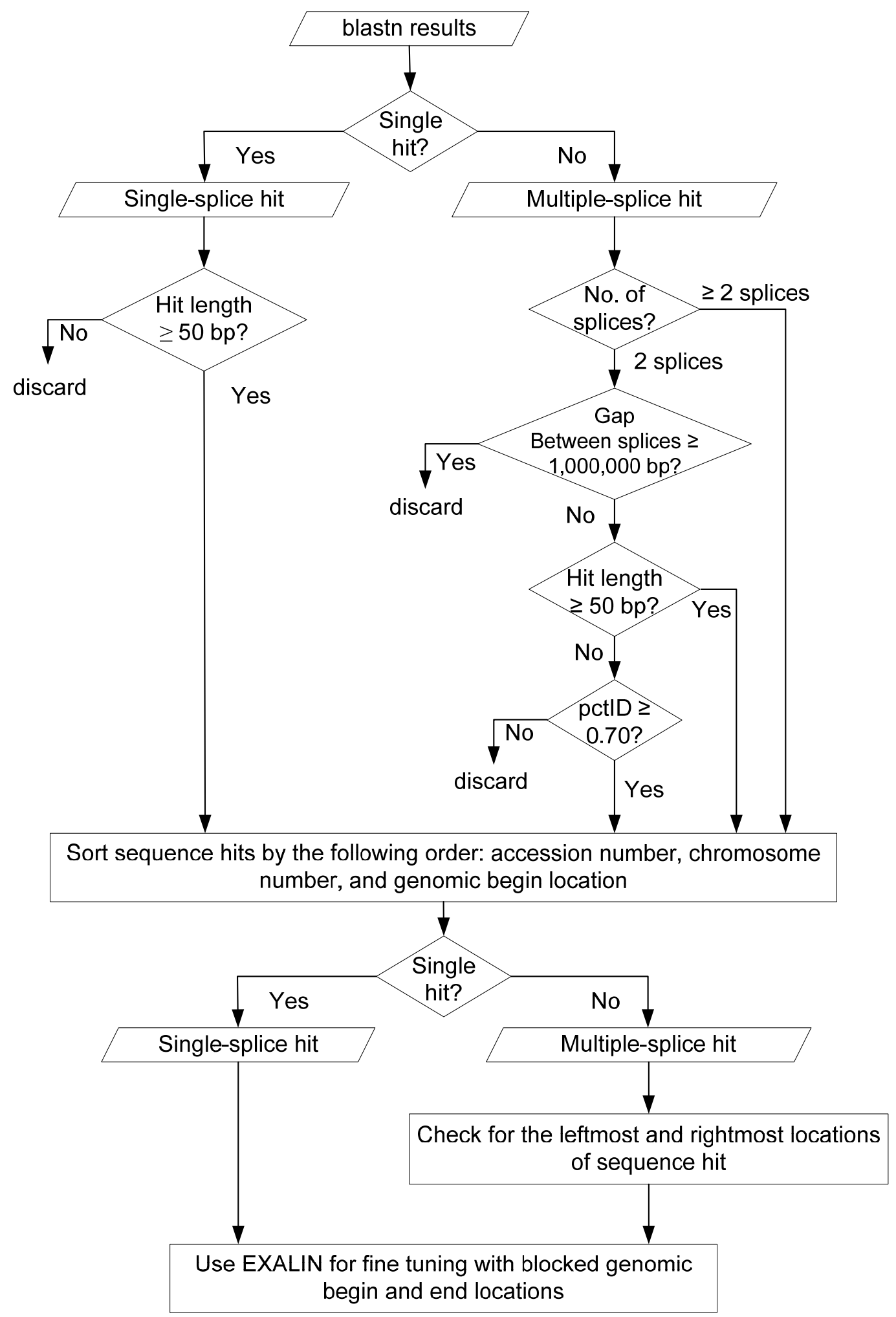

Figure 5.10: Flow chart for filtering blastn results. 
In Section 3.3.5, EXALIN (118) was discussed as a powerful method for determining sequence similarity and splice site. EXALIN can also align sequences blocked within a given range in the genome. After blastn results were filtered based on size and percent identity and sorted according to the genomic begin location, the hit is further refined to determine the leftmost and rightmost locations in genome in order to set up a searchable boundary in EXALIN. If the blocked range is over 1,000,000 bases, the search will be broken into pieces with a size of 500,000 bases. Otherwise, both boundaries will be extended to the closest thousand number. For instance, the hit after running blastn for GI 5555714 to C. elegans chromosome III were split into two pieces: 5971574-5971723 and 5972503-5972622. To use EXALIN for fine tuning, the search range will be 5971571-5972622. To extend both ends to the closest thousand number, it is actually comparing 5555714 against chromosome III between the genomic locations 5971000-5973000 when running EXALIN.

The comparison results calculated by EXALIN to find exon/intron junctions are then split into two groups: single-splice hit and multiple-splice hit. If it is a single-splice hit, sequences of length $<50 \mathrm{bp}$ are discarded. If it is a multiple-splice hit, the number of segments, the left and right ends of each segment is recorded. All the single-splice and multiple-splice hits are merged and sorted in these order: accession number, chromosome number and genomic begin location.

Before processing fine tuning in sequence comparison, the searchable boundary for running EXALIN is based on the blastn result. Thus, if the two blocked boundaries in the same chromosome generated by blastn results are different, ideally EXALIN should return different computational outcomes. For example, blastn returns two 
groups of HSP after comparing GI no. 18288550 against chromosome V for C. elegans

(Figure 5.11). The boundaries found by blastn were 2230674-9303595 and 2230017-

5705627, respectively, and the EXALIN blocked boundaries are 2230000-9304000 and

2230000-5706000 (Figure 5.12). Figures 5.12 and 5.13 show the results returned by

EXALIN that is differing from what blastn has generated in Figure 5.11.

\begin{tabular}{|c|c|c|c|c|c|c|c|c|c|c|}
\hline \multicolumn{11}{|c|}{$\begin{array}{l}=g i|18288550| \mathrm{g} \\
(722 \text { letters })\end{array}$} \\
\hline$>\operatorname{chrV}$ & 212 & 2230674 & 2230764 & 31 & 121 & 10 & $(12)-15-16-13-14$ & 0.70 & 0.13 & $\odot .09$ \\
\hline$>\operatorname{chrV}$ & 92 & 2713536 & 2713593 & 263 & 320 & 10 & $12-(15)-16-13-14$ & 0.62 & $\odot . \odot 8$ & 0.05 \\
\hline$>\operatorname{chrV}$ & 86 & 8716204 & 8716231 & 331 & 358 & 10 & $12-15-(16)-13-14$ & 0.79 & $\odot . \odot 4$ & 0.03 \\
\hline$>\operatorname{chrV}$ & 199 & 8971491 & 8971708 & 335 & 552 & 10 & $12-15-16-(13)-14$ & $\odot .55$ & 0.30 & 0.16 \\
\hline$>$ chrV & 97 & 9303573 & 9303595 & 562 & 584 & 10 & $12-15-16-13-(14)$ & 0.91 & 0.03 & 0.03 \\
\hline$>\operatorname{chrV}$ & 616 & 2230017 & 2230141 & 722 & 598 & 1 & $(18)-17-19$ & $\odot .99$ & 0.17 & 0.17 \\
\hline$>\operatorname{chrV}$ & 2938 & 2230196 & 2230793 & 599 & 2 & 1 & $18-(17)-19$ & 0.99 & 0.83 & 0.82 \\
\hline >chrv & 99 & 5705583 & 5705627 & 78 & 34 & 1 & $18-17-(19)$ & 0.69 & 0.06 & $\odot . \odot 4$ \\
\hline
\end{tabular}

Figure 5.11: Sample blastn result GI no. 18288550.

(partial result sorted by genomic begin location)

Column 1, chromosome number; column 2, high-scoring pair score; columns 3 and 4, begin and end locations in genomic regions; columns 5 and 6, begin and end locations in ESTs; column 7, group; column 8 , links; columns 9, 10 and 11, percent identity, percent coverage and match value.

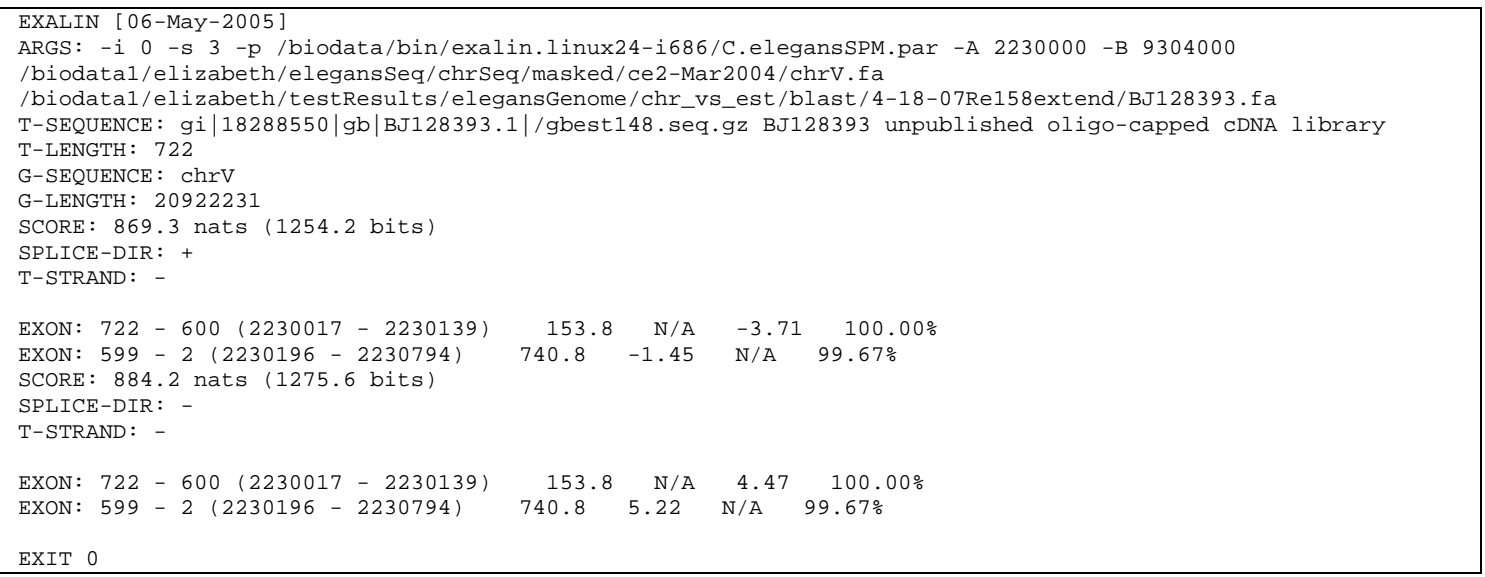

Figure 5.12: Sample EXALIN result for GI 18288550.

(Blocked search range is 2230000-9304000.) 


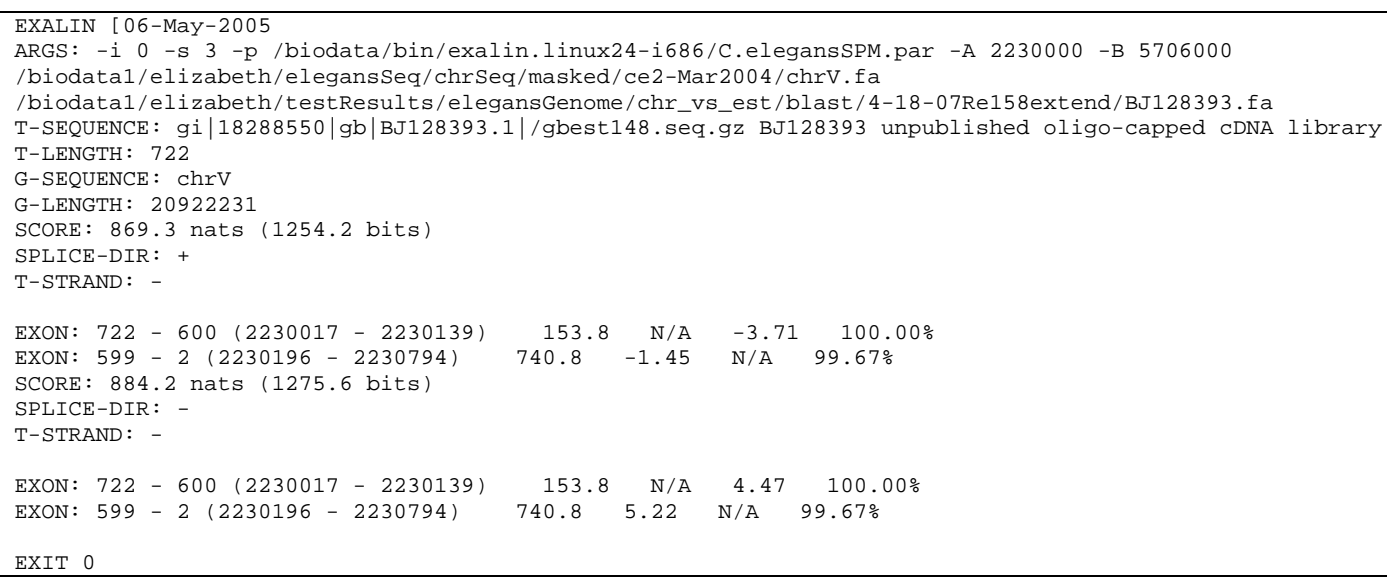

Figure 5.13: Sample EXALIN result for GI 18288550.

(Blocked search range is 2230000-5706000.)

After the fine tuning process using EXALIN, duplicate results are removed.

Figure 5.14 gives a flow chart of the process to remove redundant records generated by EXALIN.

The records are further reviewed and split into two groups: records which hit single locations and records which hit multiple locations. If the same EST accession number hits multiple locations, either in the same or different chromosome, it will be assigned into the group whose records hit multiple locations. Otherwise, it is assigned into the group whose records hit single location. Because the sequence only hits the genome once, it is highly believed to be a gene itself or can be a disabled pseudogene. Disabled pseudogenes cannot be detected using this approach. The information will be saved for further usage. Records belonging to the group whose records hit multiple locations are potentially pseudogenes. 


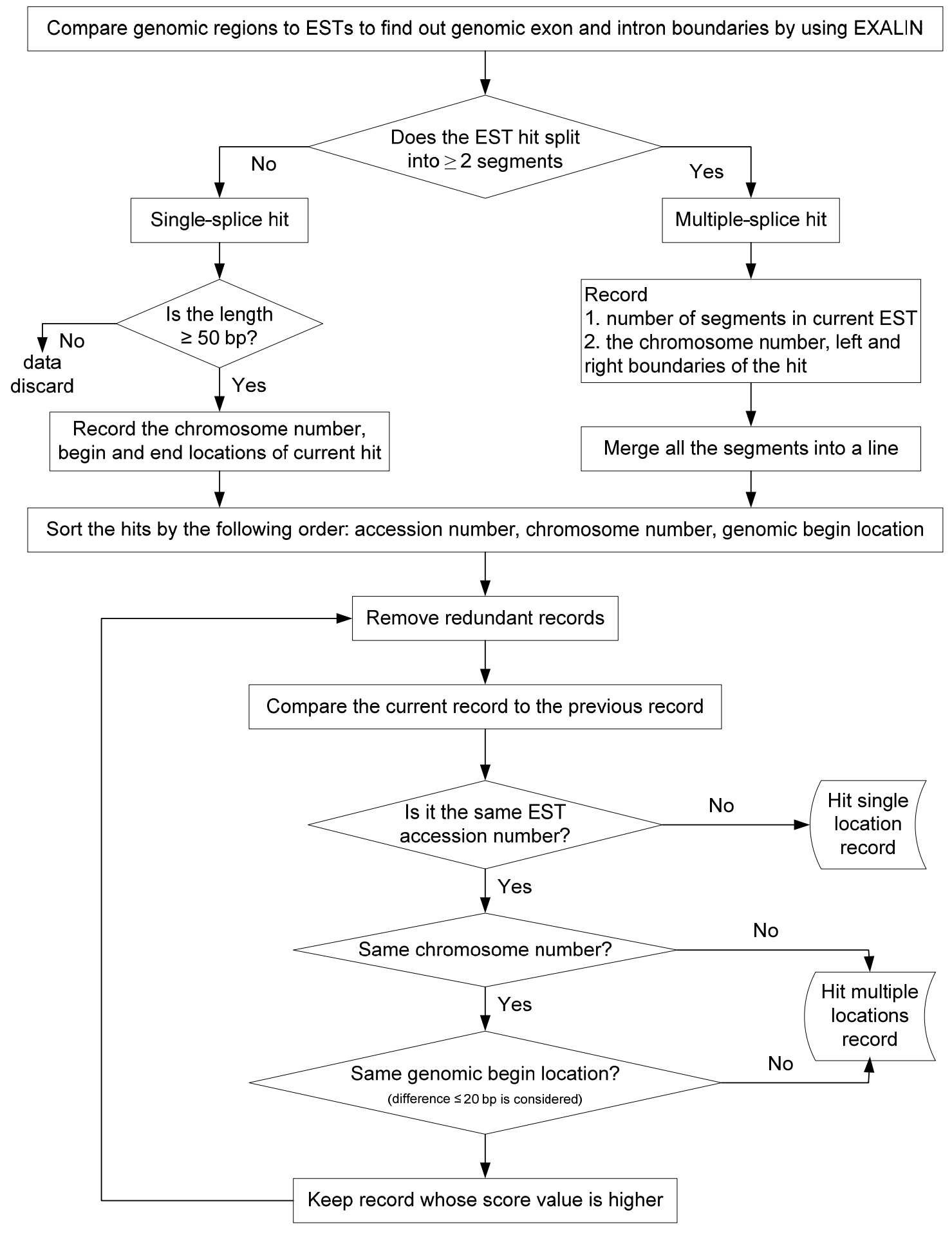

Figure 5.14: Flow chart for removing EXALIN redundant records. 


\subsubsection{Clustering}

The purpose of clustering is to incorporate overlapping ESTs that tag the same transcript of the same gene in a single cluster. Thus, measuring the similarity between any two sequences is used to cluster sequences into suitable groups. It requires a simple Boolean measurement: accept (yes) or reject (no) two sequences in the same cluster.

Multiple-location records gathered from fine tuning are further sorted by chromosome number and genomic begin location. The sequence hits are then reviewed in a "DNA walking" method: the sequence hit is reviewed base to base. If the sequences overlap each other, they are grouped together and the leftmost and rightmost boundaries will be the left end of the first sequence and the right end of the second sequence (Figure 5.15). Both single-splice and multiple-splice hits are reviewed in the same format. The ESTs belong to the same cluster will be recorded in the table for information tracing. The only exception is when a distance between left and right boundaries of a record is over 500,000 bases will be assigned in an individual group. In such group, each segment of the hit will be examined by size of the segment, distance between segments, begin and end location of the hit in EST. 


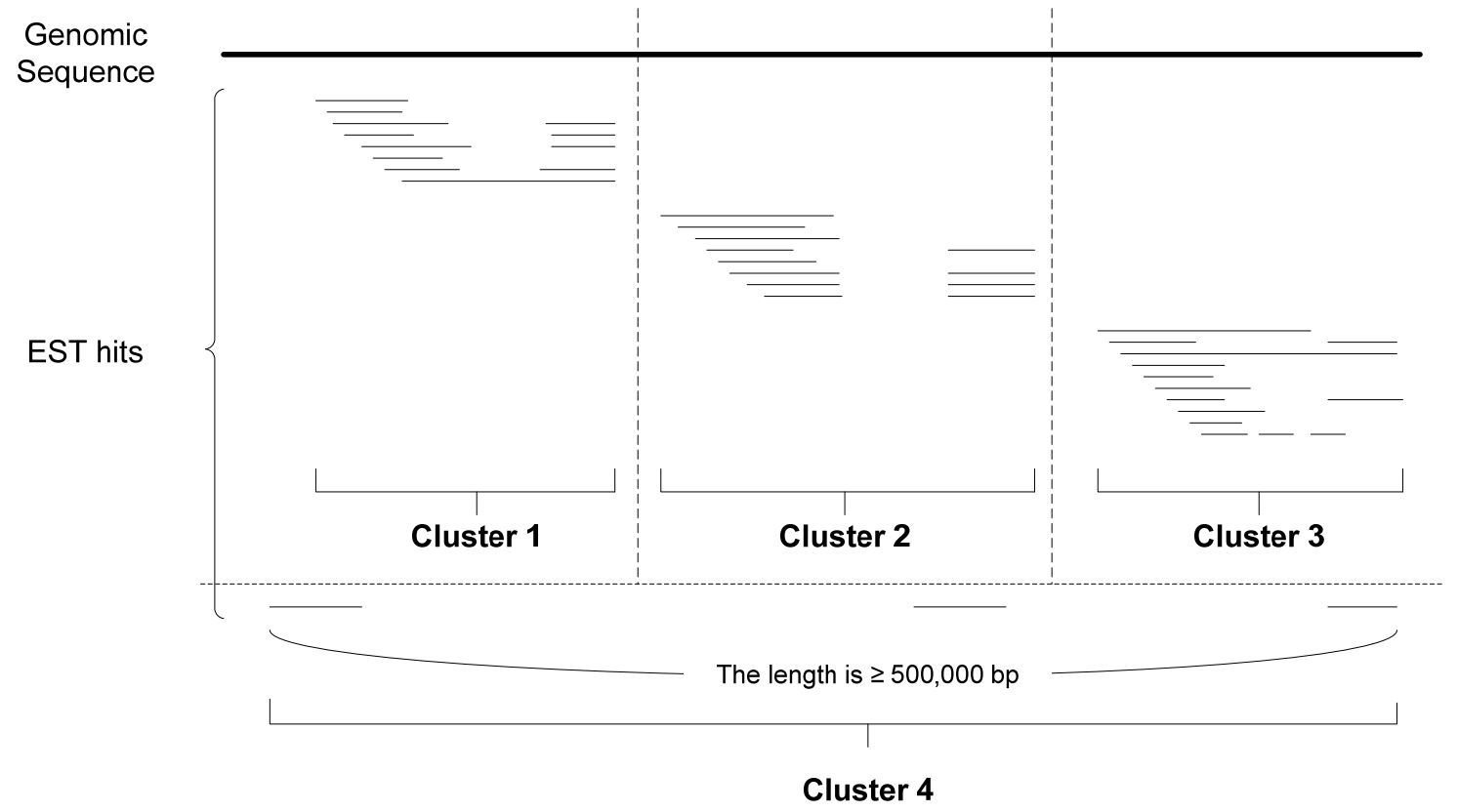

Figure 5.15: Clustering by DNA walking.

\subsubsection{Pseudogene Detection and Characterization}

After clustering EST hits into groups, each cluster will contain information pertaining to the left and right ends in genomic regions, length of the cluster and the number of ESTs in the cluster. Clusters containing gap(s) will be marked.

The left and right boundaries of each cluster are used to retrieve the corresponding genomic sequences. Each sequence is run against the RefSeq protein databases using blastx and processed to get scores for both forward and reverse directions. Each factor discussed in Section 5.3 will be examined:

1. Look for start codon (ATG),

2. Look for stop codon(s) (TGA, TAG and TAA) in open reading frames,

3. Search for poly-A signal,

4. Add up the number of adenine (A) in the poly-A tail if found, 
5. Calculate $\mathrm{G}+\mathrm{C}$ composition, and

6. Calculate values for similar synonymous mutations, synonymous mutations and nonsynonymous mutations.

\subsection{Results}

In order to demonstrate the effectiveness of this approach for pseudogene detection, a set of cytochrome ${ }^{32}$ gene and pseudogene sequences from the human genome were considered. Thirteen sequences, including one known gene and twelve pseudogenes were retrieved from different chromosomal regions and the size of sequences ranges from $\sim 1,000$ to $\sim 2,700 \mathrm{bp}$. The percentages of $\mathrm{N}$ and $\mathrm{G}+\mathrm{C}$ composition have been calculated (Table 5.5). If the Ns are included in sequences, the percentage of $\mathrm{G}+\mathrm{C}$ composition ranges from $26.23 \%$ to $41.45 \%$. If Ns are not counted in sequences, the percentage of $\mathrm{G}+\mathrm{C}$ composition ranges from $33.78 \%$ to $44.62 \%$.

\footnotetext{
${ }^{32}$ Cytochrome is a class of hemoprotein. The major biological function is for electron transport by virtue of a reversible valency change of the heme iron.
} 
Table 5.5: Percentage of $N$ and $G+C$ Composition in Sequences

\begin{tabular}{lrrrrrr}
\hline estGrouplD & length & \multicolumn{1}{c}{ N } & pctN & G+C & pctG+C & \\
\hline BC005299-1 & 2427 & 0 & $0.00 \%$ & 1006 & $41.45 \%$ & $(41.45 \%)$ \\
BC005299-2 & 1564 & 314 & $20.08 \%$ & 442 & $28.26 \%$ & $(35.36 \%)$ \\
BC005299-3 & 1219 & 0 & $0.00 \%$ & 413 & $33.88 \%$ & $(33.88 \%)$ \\
BC005299-4 & 1237 & 4 & $0.32 \%$ & 425 & $34.36 \%$ & $(34.47 \%)$ \\
BC005299-5 & 1210 & 0 & $0.00 \%$ & 419 & $34.63 \%$ & $(34.63 \%)$ \\
BC005299-6 & 1215 & 3 & $0.25 \%$ & 413 & $33.99 \%$ & $(34.08 \%)$ \\
BC005299-7 & 2686 & 436 & $16.23 \%$ & 957 & $35.63 \%$ & $(42.53 \%)$ \\
BC005299-8 & 1247 & 0 & $0.00 \%$ & 433 & $34.72 \%$ & $(34.72 \%)$ \\
BC005299-9 & 1216 & 0 & $0.00 \%$ & 414 & $34.05 \%$ & $(34.05 \%)$ \\
BC005299-10 & 1548 & 346 & $22.35 \%$ & 406 & $26.23 \%$ & $(33.78 \%)$ \\
BC005299-11 & 1784 & 233 & $13.06 \%$ & 692 & $38.79 \%$ & $(44.62 \%)$ \\
HUMCYCPS1-1 & 1060 & 0 & $0.00 \%$ & 379 & $35.75 \%$ & $(35.75 \%)$ \\
HUMCYCPS1-2 & 1366 & 310 & $22.69 \%$ & 389 & $28.48 \%$ & $(36.84 \%)$ \\
\hline
\end{tabular}

Column 1, estGroup; column 2, sequence length; column 3, number of Ns in sequence; column 4, percentage of $\mathrm{Ns}$ in sequence; column 5 , number of $\mathrm{G}+\mathrm{C}$ composition in sequence; column 6 , percent of $\mathrm{G}+\mathrm{C}$ composition in sequence; column 7, percentage of $\mathrm{G}+\mathrm{C}$ composition (without Ns) in sequence.

The start codon (ATG) and its alternative patterns (Table 5.1) were reviewed and the number of occurrences was detected. All thirteen sequences (including forward and reverse strands) contain the standard start codon ATG (Table 5.6).

The stop codons (TGA, TAG and TAA) and their alternative patterns (Table 5.2) were reviewed. Among the standard stop codon patterns, the frequency of TAA is greater than other stop patterns in most cases. The number of counts for each estGroup is listed in Table 5.7. 
Table 5.6: Frequency of Start Codon Patterns

\begin{tabular}{|c|c|c|c|c|c|c|c|c|c|c|c|c|c|c|c|}
\hline \multirow{2}{*}{ estGroupID } & \multirow{2}{*}{$\begin{array}{l}\text { ATG } \\
\text { counts }\end{array}$} & \multicolumn{2}{|c|}{ ATN } & \multicolumn{2}{|c|}{ ANG } & \multicolumn{2}{|c|}{ ANN } & \multicolumn{2}{|c|}{ NTG } & \multicolumn{2}{|c|}{ NNG } & \multicolumn{2}{|c|}{ NTN } & \multirow{2}{*}{$\begin{array}{l}\text { NNN } \\
\text { counts }\end{array}$} & \multirow[b]{2}{*}{ score } \\
\hline & & \# patterns & counts & \# patterns & counts & \# patterns & counts & \# patterns & counts & \# patterns & counts & \# patterns & counts & & \\
\hline BC005299-1F & 30 & 3 & 136 & 3 & 107 & 9 & 399 & 3 & 103 & 8 & 156 & 9 & 399 & & 4.8906 \\
\hline BC005299-1R & 41 & 3 & 124 & 3 & 116 & 9 & 387 & 3 & 132 & 8 & 243 & 9 & 379 & & 4.8906 \\
\hline BC005299-2F & 26 & 3 & 86 & 3 & 57 & 11 & 225 & 3 & 57 & 9 & 83 & 9 & 201 & 104 & 5.0781 \\
\hline BC005299-2R & 20 & 3 & 92 & 3 & 46 & 9 & 208 & 3 & 53 & 9 & 59 & 9 & 228 & 104 & 4.9531 \\
\hline BC005299-3F & 17 & 3 & 98 & 2 & 41 & 9 & 202 & 3 & 59 & 7 & 55 & 9 & 218 & & 4.5781 \\
\hline BC005299-3R & 32 & 3 & 84 & 3 & 57 & 9 & 219 & 3 & 53 & 6 & 64 & 9 & 193 & & 4.7656 \\
\hline BC005299-4F & 32 & 3 & 82 & 3 & 54 & 10 & 226 & 3 & 58 & 7 & 72 & 9 & 178 & 1 & 4.8906 \\
\hline BC005299-4R & 18 & 3 & 94 & 3 & 40 & 9 & 204 & 3 & 59 & 9 & 56 & 9 & 220 & 1 & 4.9531 \\
\hline BC005299-5F & 18 & 3 & 85 & 3 & 37 & 9 & 207 & 3 & 58 & 8 & 56 & 9 & 223 & & 4.8906 \\
\hline BC005299-5R & 20 & 3 & 84 & 2 & 54 & 9 & 226 & 3 & 62 & 9 & 74 & 8 & 186 & & 4.6406 \\
\hline BC005299-6F & 19 & 3 & 87 & 3 & 44 & 10 & 222 & 3 & 58 & 7 & 49 & 9 & 216 & 1 & 4.8906 \\
\hline BC005299-6R & 26 & 3 & 83 & 3 & 58 & 11 & 214 & 3 & 58 & 7 & 65 & 9 & 202 & 1 & 4.9531 \\
\hline BC005299-7F & 48 & 3 & 109 & 3 & 102 & 10 & 375 & 3 & 117 & 9 & 195 & 9 & 328 & 144 & 5.0156 \\
\hline BC005299-7R & 34 & 4 & 121 & 3 & 89 & 9 & 350 & 3 & 138 & 9 & 168 & 10 & 339 & 144 & 5.2656 \\
\hline BC005299-8F & 23 & 3 & 87 & 3 & 54 & 9 & 222 & 3 & 62 & 8 & 78 & 9 & 197 & & 4.8906 \\
\hline BC005299-8R & 17 & 3 & 93 & 3 & 48 & 9 & 206 & 3 & 65 & 8 & 258 & 9 & 222 & & 4.8906 \\
\hline BC005299-9F & 23 & 3 & 90 & 3 & 41 & 9 & 210 & 3 & 55 & 5 & 57 & 9 & 216 & & 4.7031 \\
\hline BC005299-9R & 24 & 3 & 89 & 3 & 53 & 9 & 217 & 3 & 63 & 6 & 64 & 9 & 194 & & 4.7656 \\
\hline BC005299-10F & 20 & 3 & 91 & 3 & 42 & 9 & 204 & 3 & 49 & 7 & 55 & 10 & 222 & 114 & 4.8906 \\
\hline BC005299-10R & 30 & 3 & 82 & 3 & 58 & 11 & 212 & 3 & 56 & 7 & 57 & 9 & 196 & 114 & 4.9531 \\
\hline BC005299-11F & 37 & 3 & 76 & 3 & 103 & 9 & 240 & 3 & 66 & 8 & 155 & 10 & 180 & 77 & 4.9531 \\
\hline BC005299-11R & 28 & 3 & 82 & 3 & 41 & 11 & 204 & 3 & 88 & 8 & 110 & 9 & 257 & 77 & 5.0156 \\
\hline HUMCYCPS1-1F & 17 & 3 & 82 & 3 & 38 & 9 & 179 & 3 & 51 & 7 & 53 & 9 & 174 & & 4.8281 \\
\hline HUMCYCPS1-1R & 28 & 3 & 69 & 3 & 47 & 9 & 178 & 3 & 48 & 3 & 66 & 9 & 172 & & 4.5781 \\
\hline HUMCYCPS1-2F & 23 & 3 & 71 & 3 & 48 & 10 & 180 & 3 & 47 & 9 & 76 & 9 & 171 & 103 & 5.0156 \\
\hline HUMCYCPS1-2R & 18 & 3 & 75 & 3 & 42 & 9 & 173 & 3 & 45 & 8 & 54 & 9 & 181 & 103 & 4.8906 \\
\hline
\end{tabular}

$F$, forward strand; $R$, reverse strand; \#, number. The patterns of start codon were listed in Table 5.1.

Formula to calculate score:

score $=$ ATG $\times 1.00+$ \# of ATN patterns $\times 0.25+\#$ of ANG patterns $\times 0.25+\#$ of ANN patterns $\times 0.0625+\#$ of NTG patterns $\times 0.25$ + \# of NNG patterns $\times 0.0625+$ \# of NTN patterns $\times 0.0625+\mathrm{NNN} \times 0.015625$ 
Table 5.7: Frequency of Stop Codon Patterns

\begin{tabular}{|c|c|c|c|c|c|c|c|c|c|c|c|c|c|c|c|}
\hline \multirow{2}{*}{ estGroupID } & \multirow{2}{*}{$\begin{array}{c}\text { TGA } \\
\text { counts }\end{array}$} & \multirow{2}{*}{$\begin{array}{l}\text { TAG } \\
\text { counts }\end{array}$} & \multirow{2}{*}{$\begin{array}{c}\text { TAA } \\
\text { counts }\end{array}$} & \multicolumn{2}{|c|}{ TGN } & \multicolumn{2}{|c|}{ TNG } & \multicolumn{2}{|c|}{ TAN } & \multicolumn{2}{|c|}{ TNA } & \multicolumn{2}{|c|}{ TNN } & \multicolumn{2}{|c|}{ NAA } \\
\hline & & & & \# patterns & counts & \# patterns & counts & \# patterns & counts & \# patterns & counts & \# patterns & counts & \# patterns & counts \\
\hline BC005299-1F & 42 & 21 & 71 & 3 & 89 & 2 & 51 & 2 & 68 & 2 & 111 & 4 & 215 & 3 & 154 \\
\hline BC005299-1R & 44 & 29 & 67 & 3 & 125 & 2 & 64 & 2 & 65 & 2 & 113 & 4 & 165 & 3 & 146 \\
\hline BC005299-2F & 23 & 16 & 44 & 3 & 56 & 2 & 27 & 2 & 45 & 2 & 69 & 4 & 86 & 3 & 89 \\
\hline BC005299-2R & 21 & 15 & 48 & 3 & 51 & 2 & 24 & 2 & 42 & 2 & 67 & 5 & 125 & 3 & 75 \\
\hline BC005299-3F & 25 & 12 & 49 & 3 & 51 & 1 & 25 & 2 & 41 & 2 & 67 & 4 & 124 & 3 & 70 \\
\hline BC005299-3R & 25 & 13 & 42 & 3 & 57 & 1 & 20 & 2 & 46 & 2 & 74 & 4 & 81 & 3 & 97 \\
\hline BC005299-4F & 26 & 14 & 40 & 3 & 61 & 2 & 24 & 2 & 43 & 2 & 69 & 4 & 79 & 3 & 94 \\
\hline BC005299-4R & 28 & 13 & 41 & 3 & 49 & 2 & 25 & 2 & 45 & 2 & 66 & 4 & 126 & 3 & 76 \\
\hline BC005299-5F & 23 & 13 & 42 & 3 & 51 & 2 & 21 & 2 & 44 & 2 & 62 & 4 & 128 & 3 & 76 \\
\hline BC005299-5R & 20 & 17 & 42 & 3 & 60 & 2 & 27 & 2 & 39 & 2 & 65 & 4 & 77 & 3 & 91 \\
\hline BC005299-6F & 25 & 12 & 46 & 3 & 50 & 1 & 22 & 2 & 42 & 2 & 65 & 4 & 118 & 3 & 81 \\
\hline BC005299-6R & 23 & 15 & 42 & 3 & 58 & 2 & 27 & 2 & 40 & 2 & 71 & 4 & 85 & 3 & 87 \\
\hline BC005299-7F & 40 & 28 & 50 & 3 & 123 & 2 & 31 & 2 & 53 & 2 & 105 & 5 & 164 & 3 & 132 \\
\hline BC005299-7R & 48 & 20 & 57 & 3 & 124 & 2 & 41 & 2 & 56 & 2 & 90 & 6 & 198 & 3 & 119 \\
\hline BC005299-8F & 25 & 17 & 42 & 3 & 57 & 2 & 25 & 2 & 43 & 2 & 72 & 4 & 83 & 3 & 92 \\
\hline BC005299-8R & 25 & 10 & 47 & 3 & 45 & 2 & 23 & 2 & 45 & 2 & 67 & 4 & 124 & 3 & 78 \\
\hline BC005299-9F & 29 & 15 & 43 & 3 & 49 & 1 & 24 & 2 & 40 & 2 & 66 & 4 & 117 & 3 & 79 \\
\hline BC005299-9R & 24 & 13 & 42 & 3 & 60 & 2 & 21 & 2 & 43 & 2 & 72 & 4 & 89 & 3 & 88 \\
\hline BC005299-10F & 23 & 14 & 47 & 3 & 44 & 2 & 22 & 3 & 39 & 2 & 72 & 5 & 121 & 3 & 76 \\
\hline BC005299-10R & 26 & 15 & 46 & 3 & 57 & 2 & 23 & 2 & 38 & 2 & 70 & 4 & 82 & 3 & 85 \\
\hline BC005299-11F & 28 & 24 & 29 & 4 & 74 & 2 & 28 & 2 & 30 & 2 & 50 & 5 & 93 & 3 & 103 \\
\hline BC005299-11R & 29 & 20 & 21 & 3 & 85 & 2 & 32 & 2 & 45 & 2 & 47 & 4 & 167 & 3 & 78 \\
\hline HUMCYCPS1-1F & 24 & 9 & 40 & 3 & 44 & 1 & 19 & 2 & 33 & 2 & 54 & 4 & 101 & 3 & 66 \\
\hline HUMCYCPS1-1R & 23 & 11 & 31 & 3 & 50 & 2 & 19 & 2 & 39 & 2 & 64 & 2 & 78 & 3 & 75 \\
\hline HUMCYCPS1-2F & 21 & 13 & 33 & 3 & 46 & 2 & 23 & 2 & 38 & 3 & 57 & 4 & 76 & 3 & 129 \\
\hline HUMCYCPS1-2R & 19 & 12 & 38 & 3 & 44 & 2 & 19 & 2 & 35 & 2 & 54 & 5 & 100 & 3 & 64 \\
\hline
\end{tabular}

F, forward strand; R, reverse strand. The patterns of stop codon were listed in Table 5.2. 
Table 5.7: Frequency of Stop Codon Patterns (Continued)

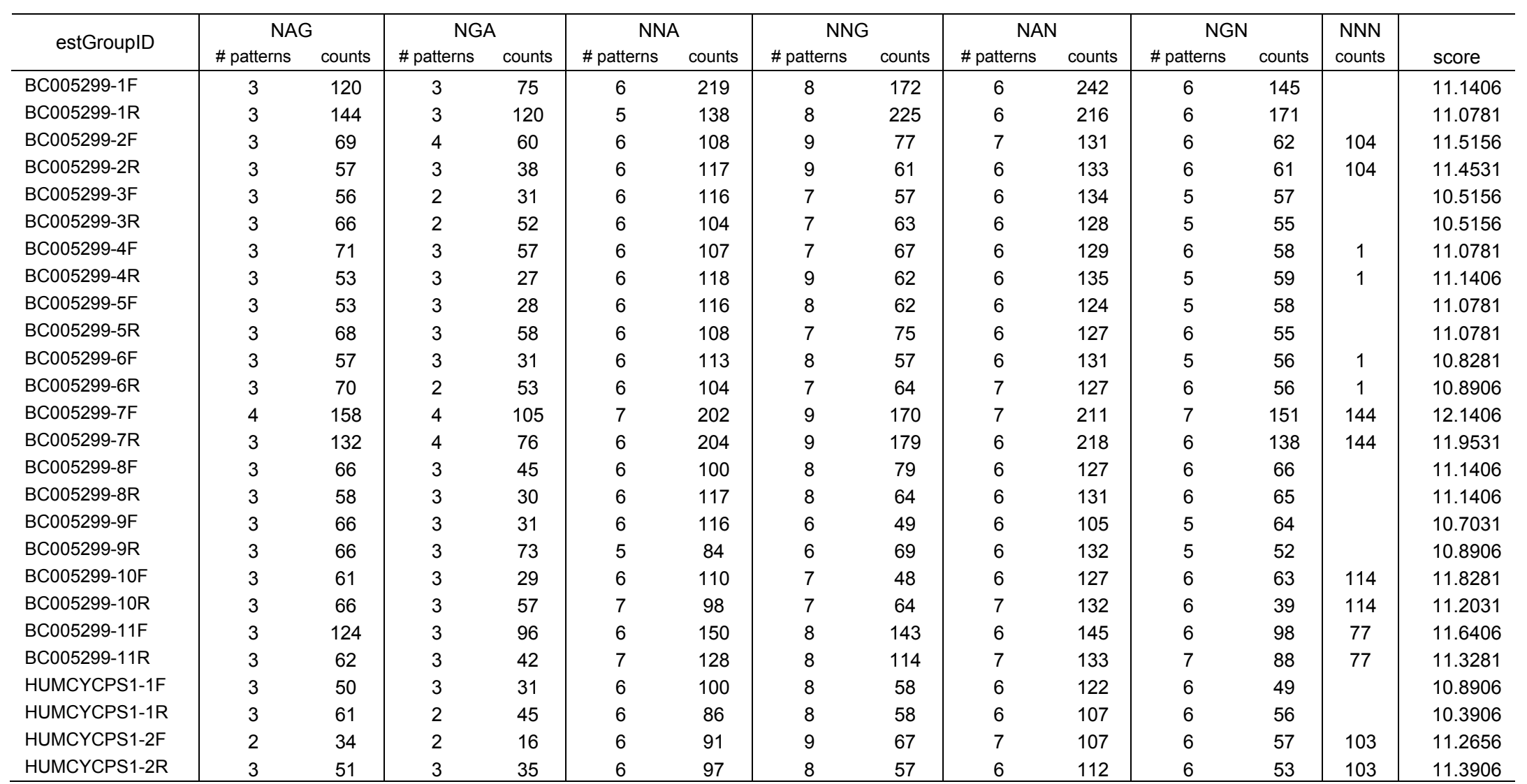

F, forward strand; $R$, reverse strand. The patterns of stop codon were listed in Table 5.2.

Formula to calculate score:

score $=$ TGA $\times 1.00+$ TAG $\times 1.00+$ TAA $\times 1.00+\#$ of TGN patterns $\times 0.25+\#$ of TNG patterns $\times 0.50$

+ \# of TAN patterns $\times 0.50+$ \# of TNA patterns $\times 0.25+$ \# of TNN patterns $\times 0.25+$ \# of NAA patterns $\times 0.25$

+ \# of NAG patterns $\times 0.25+$ \# of NGA patterns $\times 0.25+$ \# of NNA patterns $\times 0.0625$

+ \# of NNG patterns $\times 0.0625+$ \# of NAN patterns $\times 0.0625+$ \# of NGN patterns $\times 0.0625+\mathrm{NNN} \times 0.015625$ 
Table 5.8: Frequency of Poly-A Signal Patterns

\begin{tabular}{|c|c|c|c|c|c|c|c|c|c|c|c|c|c|c|c|c|c|}
\hline estGroupID & AATAAA & ATTAAA & AGTAAA & TATAAA & CATAAA & GATAAA & AATATA & AATACA & AATAGA & AAAAAG & ACTAAA & AAGAAA & AATGAA & TTTAAA & AAAACA & GGGGCT & score \\
\hline BC005299-1F & 2 & 3 & 2 & 2 & 2 & 2 & 2 & 3 & & 3 & 1 & 3 & 3 & 9 & 2 & & 6.80 \\
\hline BC005299-1R & 1 & 7 & 1 & 1 & 1 & 1 & 2 & 1 & & 3 & 2 & 2 & 1 & 9 & 3 & & 7.05 \\
\hline BC005299-2F & & 4 & 1 & & 1 & 1 & 1 & & 1 & 3 & 2 & 2 & 1 & 6 & 1 & & 3.95 \\
\hline BC005299-2R & 2 & 2 & 1 & 1 & 2 & & 2 & 2 & & 1 & 1 & & 1 & 6 & 1 & & 4.75 \\
\hline BC005299-3F & 3 & 2 & 1 & 2 & 2 & & 2 & 1 & & 1 & 1 & & 2 & 7 & 1 & & 5.95 \\
\hline BC005299-3R & 3 & & 1 & 1 & & 1 & 2 & & & 3 & 2 & 2 & 1 & 7 & & & 5.00 \\
\hline BC005299-4F & & 3 & 1 & 1 & 2 & 1 & 1 & & & 4 & & 1 & 1 & 5 & 1 & & 3.25 \\
\hline BC005299-4R & 1 & 1 & 1 & 4 & 1 & & 3 & 2 & & & 1 & 5 & & 5 & 2 & & 3.80 \\
\hline BC005299-5F & 2 & 1 & & 4 & 2 & & 3 & 1 & & 1 & 1 & & 1 & 5 & 1 & & 4.35 \\
\hline BC005299-5R & & 2 & 1 & & 1 & 1 & 1 & & 1 & 3 & 2 & 2 & 1 & 5 & 1 & & 2.85 \\
\hline BC005299-6F & 2 & 1 & 1 & 3 & 2 & & 4 & 2 & & & 1 & 1 & 2 & 6 & 1 & & 4.75 \\
\hline BC005299-6R & & 3 & 1 & & 1 & 1 & & & & 2 & & 1 & 1 & 6 & 2 & & 2.90 \\
\hline BC005299-7F & & 3 & 1 & & 1 & 1 & 1 & & 1 & 2 & & & & 4 & 1 & & 2.65 \\
\hline BC005299-7R & 1 & 1 & 1 & 4 & 3 & 4 & 2 & 2 & & 3 & 1 & & 2 & 4 & 1 & & 4.15 \\
\hline BC005299-8F & 1 & 1 & 1 & & & 2 & 2 & & 1 & 2 & & 2 & 1 & 7 & 1 & & 3.35 \\
\hline BC005299-8R & 2 & 2 & 1 & 5 & 1 & & 3 & 2 & & 1 & 1 & & 2 & 7 & 1 & & 5.35 \\
\hline BC005299-9F & 1 & 3 & 2 & 3 & 1 & & 3 & 1 & & 1 & 1 & & 1 & 5 & 1 & & 4.35 \\
\hline BC005299-9R & & 2 & 1 & & 2 & 1 & 2 & & 1 & 3 & 1 & & 2 & 5 & 1 & & 2.85 \\
\hline BC005299-10F & 1 & 2 & & 5 & 2 & & 2 & 2 & & 1 & 1 & & 2 & 5 & 2 & & 4.10 \\
\hline BC005299-10R & & 3 & 1 & 1 & 1 & 1 & 1 & 1 & 1 & 3 & & 1 & 1 & 5 & 1 & & 3.25 \\
\hline BC005299-11F & 3 & & 1 & & & & 1 & 1 & 2 & & 1 & & & 2 & 3 & & 3.95 \\
\hline BC005299-11R & & & & & 1 & & 4 & & 2 & 1 & & 1 & & 1 & 1 & 1 & 1.10 \\
\hline HUMCYCPS1-1F & 3 & 2 & 1 & 2 & 2 & & 2 & 1 & & 1 & 1 & & 3 & 5 & 1 & & 5.85 \\
\hline HUMCYCPS1-1R & & 2 & 1 & & & 1 & 1 & & & 2 & 1 & 1 & 1 & 5 & & & 2.30 \\
\hline HUMCYCPS1-2F & & 2 & 1 & & & & 1 & & 1 & 2 & 1 & 1 & 1 & 4 & 1 & & 2.25 \\
\hline HUMCYCPS1-2R & 2 & 2 & 1 & 1 & 2 & & 2 & 1 & & 1 & 1 & & 1 & 4 & 1 & & 4.45 \\
\hline & 30 & 54 & 25 & 40 & 33 & 18 & 50 & 23 & 11 & 47 & 24 & 25 & 32 & 139 & 32 & 1 & \\
\hline
\end{tabular}

Formula to calculate score:

score $=$ \# of AATAAA $\times 1.00+$ \# of ATTAAA $\times 0.50+$ \# of AGTAAA $\times 0.1+$ \# of TATAAA $\times 0.1+\#$ of GATAAA $\times 0.1$

+ \# of AATATA $\times 0.1+\#$ of AATACA $\times 0.1+\#$ of AATAGA $\times 0.1+\#$ of AAAAAG $\times 0.1+\#$ of ACTAAA $\times 0.1$

+ \# of AAGAAA $\times 0.1+$ \# of AATGAA $\times 0.1+$ \# of TTTAAA $\times 0.1+\#$ of AAAACA $\times 0.05+\#$ of GGGGCT $\times 0.05$ 
Table 5.9: Poly-A Site Information for Each Sequence

\begin{tabular}{lrrrrrrrr}
\hline estGrouplD & length & $\begin{array}{c}\text { start } \\
\text { codon } \\
\text { location }\end{array}$ & $\begin{array}{c}\text { stop } \\
\text { codon }\end{array}$ & $\begin{array}{c}\text { stop } \\
\text { codon } \\
\text { location }\end{array}$ & $\begin{array}{c}\% \text { finding } \\
\text { poly-A site }\end{array}$ & $\begin{array}{c}\text { strand } \\
\text { direction }\end{array}$ & $\begin{array}{c}\text { poly-A } \\
\text { site }\end{array}$ & $\begin{array}{c}\text { poly-A } \\
\text { site } \\
\text { location }\end{array}$ \\
\hline BC005299-1 & 2427 & 1140 & TAA & 1556 & $59.25 \%$ & Reverse & ATTAAA & 2137 \\
BC005299-2 & 1564 & 68 & TAA & 698 & $38.18 \%$ & Forward & ATTAAA & 1272 \\
BC005299-3 & 1219 & 27 & TAA & 342 & $29.76 \%$ & Reverse & ATTAAA & 919 \\
BC005299-4 & 1237 & 64 & TAA & 382 & $30.20 \%$ & Forward & ATTAAA & 1160 \\
BC005299-5 & 1210 & 17 & TAA & 323 & $29.54 \%$ & Reverse & ATTAAA & 1114 \\
BC005299-6 & 1215 & 21 & TTG & 333 & $29.66 \%$ & Reverse & ATTAAA & 1120 \\
BC005299-8 & 1247 & 65 & TAC & 368 & $30.44 \%$ & Forward & AGTAAA & 585 \\
BC005299-9 & 1216 & 17 & TAC & 315 & $29.69 \%$ & Reverse & ATTAAA & 1123 \\
BC005299-10 & 1548 & 27 & TAC & 318 & $37.79 \%$ & Reverse & ATTAAA & 1455 \\
HUMCYCPS1-1 & 1060 & 76 & TAA & 391 & $25.88 \%$ & Reverse & ATTAAA & 968 \\
HUMCYCPS1-2 & 1366 & 70 & TAA & 700 & $33.35 \%$ & Forward & ATTAAA & 1274 \\
\hline
\end{tabular}


Sixteen patterns of poly-A signals were reviewed as well. The number of times each of the patterns was occurred were accumulated and listed in Table 5.8. In most cases, AATAAA, ATTAAA and AGTAAA were found. CATAAA was only found in BC005299-11 (marked in blue). In addition, that was the only sequence found the GGGGCT poly-A signal (marked in pink).

After running blastx, BC005299-7 and BC005299-11 were found to relate to other protein sequences. These two sequences were removed from pseudogene candidates. Based on the blastx results, the strand direction for each sequence was found. In addition, the start and stop codon locations for remain eleven sequences were recognized. Since the poly-A site is a six-base pattern, it has $4^{6}=4096$ combinations. The percentage of finding a poly-A signal is calculated $(\%$ of finding poly-A site $=$ sequence length $\div$ 4096). With the information where the stop codons were located, the poly-A sites were found in the 3' UTR. It is surprising to find that ten out of eleven sequences have ATTAAA as the poly-A sites. Only BC005299-8 has AGTAAA as its poly-A signal. None of the sequences has found the most common poly-A signal AATAAA in the $3^{\text {' }}$ UTR, using the stop codon location as a reference (Table 5.9). In addition, seven out of thirteen sequences have found TAA as stop codons. One has TTG and three have TAC as stop codons.

Based on the comparison results provided by blastx, the nucleotide sequences of each estGroup were retrieved. Each was compared to the cytochrome sequence at the nucleotide level in order to calculate the number of mutational events (similar synonymous, synonymous and nonsynonymous mutations) defined in Section 5.3.5. The similar synonymous mutation is marked with lower case ' $s$ '. The synonymous mutation is 
marked with upper case ' $\mathrm{S}$ '. The nonsynonymous mutation is marked with upper case ' $N$ '. In addition, if a gap was inserted in the blastx alignment result, either in the subject or query sequence, the number of gaps for each estGroup was also counted. If the insertion occurs in the subject sequence, it is marked with caret $\operatorname{sign}^{` \wedge}$. If the insertion occurs in the query sequence, it is marked with minus sign '-' sign. If a premature stop codon was found in the query sequence, the nonsynonymous mutation was marked as lower case ' $p$ '. But if a premature stop codon was found in the query sequence and there is an insertion found in the subject sequence, the nonsynonymous mutation was marked as lower case ' $q$ '. In addition, the number of non-mutational nucleotide is marked as a blank ' '. The results are shown in Table 5.10.

Table 5.10: Counts of Similar Synonymous Mutations, Synonymous Mutations, Nonsynonymous Mutations, and Insertion for Each Sequence

\begin{tabular}{l|cccccccc|c}
\hline \multicolumn{1}{c|}{ estGroupID } & $\mathrm{N}$ & $\mathrm{S}$ & $\mathrm{s}$ & - & $\wedge$ & $\mathrm{p}$ & $\mathrm{q}$ & & \\
\hline BC005299-1 & 0 & 0 & 0 & 0 & 0 & 0 & 0 & 315 & 315 \\
BC005299-2 & 7 & 3 & 2 & 0 & 0 & 0 & 0 & 303 & 315 \\
BC005299-3 & 5 & 5 & 0 & 0 & 0 & 0 & 0 & 305 & 315 \\
BC005299-4 & 11 & 10 & 5 & 0 & 0 & 0 & 0 & 289 & 315 \\
BC005299-5 & 11 & 7 & 5 & 9 & 0 & 0 & 0 & 283 & 315 \\
BC005299-6 & 18 & 6 & 5 & 3 & 0 & 2 & 0 & 281 & 315 \\
BC005299-8 & 14 & 6 & 9 & 3 & 0 & 1 & 0 & 273 & 306 \\
BC005299-9 & 15 & 5 & 12 & 0 & 0 & 0 & 0 & 262 & 294 \\
BC005299-10 & 52 & 6 & 13 & 15 & 0 & 0 & 0 & 220 & 306 \\
HUMCYCPS1-1 & 5 & 5 & 0 & 0 & 0 & 0 & 0 & 305 & 315 \\
HUMCYCPS1-2 & 7 & 3 & 2 & 0 & 0 & 0 & 0 & 303 & 315 \\
\hline
\end{tabular}

$\mathrm{N}$, nonsynonymous mutation; S, synonymous mutation; s, similar synonymous mutation;

-, insertion in query sequence; $\wedge$, insertion in subject sequence; $p$, nonsynonymous mutation when a stop codon appears in the query sequence; $q$, insertion occurs in the subject sequence when a stop codon occurs in the query sequence; blank ' ', the number of non-mutational nucleotides; the last column represents the total number of nucleotides within the sequence. 
In Section 5.3.3, a poly-A tail was defined as having a length of ten or more adenine (A) residues. However, the computational program did not return results for eleven candidate sequences. It is possible that none of the sequences have a poly-A tail. It is necessary to study the sequence file manually in order to check if the sequence does not have a poly-A tail in the 3' UTR. In fact, there are poly-A tails in the 3' UTR. However, the longest size of adenine (A) residues is seven bases. Since the program requires having ten adenines (A) as a minimum number, if a sequence pattern is like AAAAAAAGAAA, the program will not count it as a poly-A tail because the nucleotide $\mathrm{G}$ has broken the length. Therefore, eight out of eleven pseudogene candidate sequences have poly-A tails, after reviewing the sequence file. The size of poly-A tails ranges from eleven to 29 bases (Table 5.11).

A score was generated and each pseudogene factor was assigned a value as defined in Section 5.3. Table 5.11 provides the scoring results for each candidate pseudogene sequence. The unit score for each column is shown in the bottom of the table. The score for start codon, stop codon and poly-A sites have been defined in Tables 5.1 to 5.3. For poly-A tails, if the size is less than twenty adenines (A), each base is giving a 0.1. If the poly-A tail has more than twenty adenines (A), then 2.0 is given. 
Table 5.11: Score for Pseudogene Candidate Sequences

\begin{tabular}{|c|c|c|c|c|c|c|c|c|c|c|c|c|c|}
\hline estGroupID & $\begin{array}{c}\text { start } \\
\text { codon }\end{array}$ & $\begin{array}{l}\text { stop } \\
\text { codon }\end{array}$ & $\begin{array}{l}\text { poly-A } \\
\text { site }\end{array}$ & $\begin{array}{c}\text { poly-A } \\
\text { tails }\end{array}$ & $\mathrm{N}$ & $S$ & $\mathrm{~s}$ & - & $\wedge$ & $p$ & $q$ & & score \\
\hline BC005299-1 & ATG & TAA & ATTAAA & 0 & 0 & 0 & 0 & 0 & 0 & 0 & 0 & 315 & 5.65 \\
\hline BC005299-2 & ATG & TAA & ATTAAA & 15 & 7 & 3 & 2 & 0 & 0 & 0 & 0 & 303 & 7.86 \\
\hline BC005299-3 & ATG & TAA & ATTAAA & 23 & 5 & 5 & 0 & 0 & 0 & 0 & 0 & 305 & 8.10 \\
\hline BC005299-4 & ATG & TAA & ATTAAA & 15 & 11 & 10 & 5 & 0 & 0 & 0 & 0 & 289 & 8.34 \\
\hline BC005299-5 & ATG & TAA & ATTAAA & 13 & 11 & 7 & 5 & 9 & 0 & 0 & 0 & 283 & 8.95 \\
\hline BC005299-6 & ATG & TTG & ATTAAA & 11 & 18 & 6 & 5 & 3 & 0 & 2 & 0 & 281 & 8.27 \\
\hline BC005299-8 & ATG & TAC & AGTAAA & 26 & 14 & 6 & 9 & 3 & 0 & 1 & 0 & 273 & 8.64 \\
\hline ВC005299-9 & ATG & TAC & ATTAAA & 29 & 15 & 5 & 12 & 0 & 0 & 0 & 0 & 262 & 8.77 \\
\hline BC005299-10 & ATG & TAC & ATTAAA & 29 & 52 & 6 & 13 & 15 & 0 & 0 & 0 & 220 & 13.61 \\
\hline HUMCYCPS1-1 & ATG & TAA & ATTAAA & 0 & 5 & 5 & 0 & 0 & 0 & 0 & 0 & 305 & 6.10 \\
\hline HUMCYCPS1-2 & ATG & TAA & ATTAAA & 0 & 7 & 3 & 2 & 0 & 0 & 0 & 0 & 303 & 6.36 \\
\hline unit score & Table 5.1 & Table 5.2 & Table 5.3 & $1.0 \sim 2.0$ & 0.1 & 0.01 & 0.05 & 0.1 & 0.05 & 0.1 & 0.05 & 0.01 & \\
\hline
\end{tabular}

$\mathrm{N}$ : nonsynonymous mutation; S: synonymous mutation; s: similar synonymous mutation; -: gap insertion in the query sequence; ^: gap insertion in the subject sequence; $p$ : nonsynonymous mutation occurred to make the coding codon becomes stop codon in query sequence; gap insertion in the subject sequence because stop codon is found in query sequence.

Formula to calculate score: \#, number

score $=$ start codon + stop codon + poly-A site + score of poly-A tail $(1.0 \sim 2.0)+\#$ of N's $\times 0.1+\#$ of S's $\times 0.01$

$$
+\# \text { of s's } \times 0.05+\# \text { of }- \text { 's } \times 0.1+\# \text { of }{ }^{\wedge} \text { 's } \times 0.05+\# \text { of p's } \times 0.1+\# \text { of q's } \times 0.05+\# \text { of blank ' ' } \times 0.01
$$




\subsection{Discussion}

BC005299-1 was retrieved from chromosome 7. It is the only sequence that does not contain any mutation in the 315-base aligning to the cytochrome protein sequence, when running blastx. The blastx returned the alignment results in two pieces of $100 \%$ sequence identity, with a gap of 100 bases. It is most likely to be a gene itself. In fact, these 100 bases represent an intron.

BC005299-2 and HUMCYCPS1-2 are another two sequences whose blastx results return two pieces. Both of them were retrieved from chromosome 6. After reviewing the sequences themselves, the sequences in between the two segments are mainly Ns. The size ranges from $\sim 50$ to $\sim 310$ bases. In fact, both sequences were retrieved from overlapping regions in chromosome 6 with a $\sim 1100$ - base overlap. BC005299-3 and HUMCYCPS1-1 yield similar blastx results. Both of them came from chromosome 8 . The only difference is that $\mathrm{BC} 005299-3$ has a poly-A tail but HUMCYCPS1-1 does not. After reviewing the sequences in genome, they were found have a $\sim 1000$-base overlap. BC005299-9 which was retrieved from chromosome 2 is another sequence that has four bases inserted between two segments. Otherwise, the rest of five sequences are all in one piece. The size ranges from 291 to 315.

Besides BC005299-1, the sequence similarity for the rest of the sequences in Table 5.11 ranges from $63 \%$ to $95 \%$. All of them contain poly-A signals. Eight out of the ten sequences have poly-A tails. BC005299-10 from chromosome 3 has been found the most number of nonsynonymous mutations. In addition, when compared to the cytochrome protein sequence, the sequence has to carry more gaps in order to receive the 
best scoring results. BC005299-6 from chromosome 8 and BC005299-8 from chromosome 9 have premature stop codons.

The nonsynonymous mutations (marked as upper case ' $\mathrm{N}$ ' in Table 5.11) are assigned a higher score $(0.1)$ than the synonymous mutation (marked as upper case ' $\mathrm{S}$ ' and lower case 's', scored 0.05) because of corresponding changes to the amino acid. Similar synonymous mutations have higher score $(0.05)$ than the synonymous mutation (0.01). Although the amino acid is changed to a close genetic code, the amino acid is not the same one anymore. If the error occurs again when sequences are duplicated, the correct nucleotide(s) would not only be copied.

Insertions occurred in the query sequence in blastx result (marked as minus sign '-`) are assigned a higher score (0.1) than the insertion in subject sequence (marked as caret sign '^’, scored 0.05 ). Gap insertions are only for the computational purpose in order to calculate the best comparison result. In addition, when comparison made between the query and subject sequence, if gaps are inserted to the query sequence, there are a number of nucleotides deleted in the query sequence.

The premature stop codon which is found in the query sequence causes the nonsynonymous mutation (marked as lower case ' $p$ '). It gets assigned a score of 0.1 . If the premature stop codon is found in the query sequence, but the subject sequence is inserted a gap in order to get a better score in sequence comparison, this nonsynonymous mutation (marked as lower case 'q') has a score of 0.05 .

The scores for start codons, stop codons and poly-A sites are given in Tables 5.1 to 5.3. After adding up single score for each sequence, the score ranges from 5.65 to 13.61. BC005299-1, the cytochrome gene itself has the lowest score 5.65. BC005299-10 
has the highest score due to having the highest number of nonsynonymous mutations occurring in the sequence. 


\section{CHAPTER VI}

\section{CONCLUSIONS AND DIRECTIONS FOR FUTURE RESEARCH}

To understand gene regulation and expression, expressed sequence tags (ESTs) have been used widely in sequence alignment. This dissertation has discussed the implementation of using EST sequences for finding the efficiency of current BLAST tools, determining undetected alternative splicing events, and detecting and characterizing pseudogenes in genomic sequences in Chapters 3 to 5. These projects yield many interesting research directions to explore upon completion of doctoral work. A few of these are described in Sections 6.1 to 6.4.

\subsection{BLAST Comparison Project}

In the BLAST comparison project (Chapter 3), ESTs serve as short sequences as both query and database targets for sequence alignment. Tests were made under default settings for all three chosen BLAST tools. In general, without knowing the sequence length and file size, WU BLAST was found to be the best for sequence comparison at a nucleotide level. Results may vary when comparing amino acid sequences. The BLAST comparison project was completed in 2004. All three search tools have since released updated versions. New features have been added to provide better and accurate search results. There are additional versions, such as PSI-BLAST (101), PHI-BLAST (102) and megaBLAST (223) in ncbiBLAST, as well as other parallel implementations (224-227). 
These were not tested, and may yield more efficient results. In addition, fine tuning the parameters may result in varying efficiencies.

\subsection{Rat Phosphodiesterase 4 (PDE4) Project}

In Chapter 4, computational detection of alternative splicing events was discussed. Different combinations of exons can produce different mRNA sequences. ESTs can provide partial information of the mRNA. Moreover, after high-throughput analysis was established, an increasing number of ESTs have been produced from different tissue types in the wet lab. In order to find alternative splicing variants, ESTs become a good resource. Phosphodiesterase 4 (PDE4) genes were chosen to study in the project in order to find the relationship between tissue specificity and isoforms. A computational method has been designed and implemented into programs to align rat ESTs to the corresponding chromosomal and mRNA sequences. In addition, the splice patterns and tissue type for each EST was examined to gain more insight into PDE4 isoforms tissue specificity.

Some undetected isoforms were found by the computational method. After using primers to sequence these patterns found in the PCR experiments, a number of novel isoforms were experimentally verified. Results are shown in Sections 4.8. Further collaboration with web lab biologists could result in confirmation of novel tissue specific isoforms. Additional primers can be designed and run in gels in order to verify more of the predicted patterns. These patterns could be further localized within tissues using in 
situ hybridization ${ }^{33}$. The complete sequence of the isoform is desired, and could be determined using techniques such as RACE sequencing ${ }^{34}$.

The methodology for finding alternative splicing events in PDE4 has been designed and implemented in several scripts. In the future, this process could be automated, allowing users to input a gene of interest. The results provided will allow for users conduct wet lab experiments for confirmation of predicted isoforms.

This PDE4 project focused in rat genome. Future extensions could look at incorporation of EST information from comparative genomes (such as mouse, chimpanzee and human) for detection of rare splicing events.

\subsection{Computational Method of Detecting Alternative Splicing Events}

The method developed for finding splicing variants concentrates on finding functional alternative splices. Future studies could distinguish non-functional splices from functional ones. However, this would require more biological information on the difference between functional and non-functional alternative splicing events.

\subsection{Computational Method for Pseudogene Detection and Characterization}

In addition to finding alternative splicing sequences in genomic regions, ESTs were also used to determine pseudogenes in genome. Pseudogenes have been defined as

\footnotetext{
${ }^{33}$ In situ hybridization is a technique in which single-stranded DNA are permitted to interact so that hybrids are formed by molecules with sufficiently similar, complementary sequences. It involves hybridizing a labeled nucleic acid in fluorescent dye to suitably prepared cells or histological sections. In situ hybridization is used particularly to look for specific transcription or localization of genes to specific chromosomes.

${ }^{34}$ RACE, or rapid amplification of cDNA ends, is a technique used in molecular biology to obtain the partial sequence of an RNA transcript found within a cell. RACE begins through RT-PCR to produce a cDNA copy of a region of the RNA transcript. An unknown end portion of a transcript is copied using a known sequence from the center of the transcript. The copied region is bounded by the known sequence, and either the 5' or 3' end.
} 
sequences which look like genes (having similar genomic features) but are not able to produce proteins as genes. Instead of using cDNA or mRNA sequences to find genes themselves in the genomic regions. ESTs can help to locate pseudogene sequences that have never been reported before. A computational method was developed to detect and characterize pseudogenes in Chapter 5. The biological factors for pseudogenes were evaluated, such as finding premature stop codons and calculating the number of synonymous and nonsynonymous mutations within sequences. A scoring scheme has been established in order to evaluate sequences or not. Based on the scoring scheme presented in Section 5.3, it would be easier for users to distinguish pseudogenes from gene sequences.

However, the developed computational method for pseudogene detection and characterization still requires manual review. Therefore, it is necessary to modify the detecting method to calculate the percentage of the adenine (A) in the sequences.

In pseudogene detection and characterization, ESTs are used to locate the candidate pseudogene locates in genomic regions. For better evaluation, having blastx results will help to locate the exact locations of start and stop codons in the candidate regions. blastx results also help to calculate the synonymous and nonsynonymous mutation with the sequences. Therefore, to detect and characterize pseudogenes, the known information from protein sequences is necessary. In addition, based on where the start codons of pseudogene candidate sequences are located, it would be necessary to retrieve either upstream or downstream sequence from the genomic sequences to further analyze for the presence of a TATA box and transcription factors. 
The methodology discussed in Chapter 5 was developed step-by-step and implemented into computer programs, including data preprocessing, sequence alignment, clustering and pseudogene evaluation. To extend the research in pseudogene detection and characterization, a pipeline process is planned from data preprocessing all the way to evaluation so pseudogene analysis can be performed in an automated fashion. The pipeline could be implemented to accommodate multiple genomes. The bottleneck in this process is currently the time it takes for sequence comparison. Future research could look into parallel and distributed computing applications to speed up this process. 


\section{REFERENCES}

1. Dunn,P.M. (2003) Gregor Mendel, OSA (1822-1884), founder of scientific genetics. Arch. Dis. Child Fetal Neonatal Ed, 88, F537-F539.

2. Fairbanks,D.J. and Rytting,B. (2001) Mendelian controversies: a botanical and historical review. Am. J Bot., 88, 737-752.

3. Orel,V. and Wood,R.J. (2000) Essence and origin of Mendel's discovery. C. R. Acad Sci III, 323, 1037-1041.

4. Weiling,F. (1991) Historical study: Johann Gregor Mendel 1822-1884. Am. J Med Genet, 40, 1-25.

5. Dahm,R. (2008) Discovering DNA: Friedrich Miescher and the early years of nucleic acid research. Hum. Genet, 122, 565-581.

6. Crick,F. (1970) Central dogma of molecular biology. Nature, 227, 561-563.

7. Avery,O.T., MacLeod,C.M. and McCarty,M. (1979) Studies on the chemical nature of the substance inducing transformation of pneumococcal types. Inductions of transformation by a desoxyribonucleic acid fraction isolated from pneumococcus type III. J Exp. Med, 149, 297-326.

8. Avery,O.T., MacLeod,C.M. and McCarty,M. (1995) Studies on the chemical nature of the substance inducing transformation of pneumococcal types. Induction of transformation by a desoxyribonucleic acid fraction isolated from Pneumococcus type III. 1944. Mol Med, 1, 344-365.

9. Chargaff,E. and Vischer,E. (1949) The composition of the desoxypentose nucleic acids of thymus and spleen. J Biol. Chem., 177, 405-416.

10. Chargaff,E. (1951) Structure and function of nucleic acids as cell constituents. Fed. Proc., 10, 654-659.

11. Watson,J.D. and Crick,F.H. (1953) Molecular structure of nucleic acids; a structure for deoxyribose nucleic acid. Nature, 171, 737-738.

12. Cornish-Bowden,A. (1985) Nomenclature for incompletely specified bases in nucleic acid sequences: recommendations 1984. Nucleic Acids Res., 13, 30213030 . 
13. Klug,W.S. and Cummings,M.R. (1991) Mendelian Genetics. Concepts of Genetics. Maxwell Macmillan International Pub. Group, pp. 44-72.

14. Matthaei,J.H., Jones,O.W., Martin,R.G. and Nirenberg,M.W. (1962) Characteristics and composition of RNA coding units. Proc. Natl Acad Sci U. S. A, 48, 666-677.

15. Nirenberg,M.W., Matthaei,J.H. and Jones,O.W. (1962) An intermediate in the biosynthesis of polyphenylalanine directed by synthetic template RNA. Proc. Natl Acad Sci U. S. A, 48, 104-109.

16. Nirenberg,M.W. (1963) The Genetic Code: II. The Molecular Basis of Life - An Introduction to Molecular Biology. pp. 206-216.

17. Karp,G. (2002) Cell and Molecular Biology - Concepts and Experiments. John Wiley \& Sons, Inc..

18. Klug,W.S. and Cummings,M.R. (1991) Synthesis of RNA and Protein: Transcription and Translation. Concepts of Genetics. Maxwell Macmillan International Pub. Group, pp. 312-342.

19. Klug,W.S. and Cummings,M.R. (1991) DNA - The Genetic Material. Concepts of Genetics. Maxwell Macmillan International Pub. Group, pp. 218-233.

20. Henikoff,S. (2002) Beyond the central dogma. Comput. Appl. Biosci., 18, 223225 .

21. Carthew,R.W. (2001) Gene silencing by double-stranded RNA. Curr Opin Cell Biol., 13, 244-248.

22. Fire,A., Xu,S., Montgomery,M.K., Kostas,S.A., Driver,S.E. and Mello,C.C. (1998) Potent and specific genetic interference by double-stranded RNA in Caenorhabditis elegans. Nature, 391, 806-811.

23. Fire,A.Z. (2007) Gene silencing by double-stranded RNA (Nobel Lecture). Angew. Chem. Int. Ed Engl., 46, 6966-6984.

24. Fire,A.Z. (2007) Gene silencing by double-stranded RNA. Cell Death. Differ., 14, 1998-2012.

25. Mello,C.C. (2007) Return to the RNAi world: rethinking gene expression and evolution. Cell Death. Differ., 14, 2013-2020.

26. Mello,C.C. (2007) Return to the RNAi world: rethinking gene expression and evolution (Nobel Lecture). Angew. Chem. Int. Ed Engl., 46, 6985-6994.

27. Sanger,F., Nicklen,S. and Coulson,A.R. (1977) DNA sequencing with chainterminating inhibitors. Proc. Natl. Acad. Sci. U. S. A., 74, 5463-5467. 
28. Maxam,A.M. and Gilbert,W. (1977) A new method for sequencing DNA. Proc. Natl Acad Sci U. S. A, 74, 560-564.

29. Kyle,R.A. and Shampo,M.A. (2005) Arne Tiselius--father of electrophoresis. Mayo Clin. Proc., 80, 302.

30. Tiselius,A. (1937) Electrophoresis of serum globulin: Electrophoretic analysis of normal and immune sera. Biochem. J., 31, 1464-1477.

31. Tiselius,A. (1937) Electrophoresis of serum globulin. I. Biochem. J., 31, 313-317.

32. Graham,C.A. and Hill,A.J.M. (2000) Introduction to DNA Sequencing. In Graham,C.A. and Hill,A.J.M. (eds.), DNA Sequencing Protocols. pp. 1-12.

33. Metzker,M.L. (2005) Emerging technologies in DNA sequencing. Genome Res., 15, 1767-1776.

34. Marziali,A. and Akeson,M. (2001) New DNA sequencing methods. Annu. Rev. Biomed. Eng., 3:195-223., 195-223.

35. Schuster,S.C. (2008) Next-generation sequencing transforms today's biology. Nat. Methods., 5, 16-18.

36. Mardis,E.R. (2008) The impact of next-generation sequencing technology on genetics. Trends Genet., 24, 133-141.

37. Expressed Sequence Tags database (http://www.ncbi.nlm.nih.gov/dbEST/). NCBI . 7-11-2000.

Ref Type: Electronic Citation

38. ESTs: Gene Discovery Made Easier

(http://www.ncbi.nlm.nih.gov/About/primer/est.html). 3-29-2004. A Science

Primer. NCBI.

Ref Type: Generic

39. Wolfsberg,T.G. and Landsman,D. (2001) Expressed Sequence Tags (ESTs). In Baxevanis,A.D. and Ouellette,B.F.F. (eds.), Bioinformatics - A Practical Guide to the Analysis of Genes and Proteins. Wiley-Interscience, pp. 283-301.

40. (2002) The Human Genome. Nature Publishing Group, New York.

41. U.S. Human Genome Project Research Goals (http://www.ornl.gov/sci/techresources/Human_Genome/hg5yp/index.shtml). Human Genome Project . 9-9-2004.

Ref Type: Electronic Citation 
42. Lander,E.S., Linton,L.M., Birren,B., Nusbaum,C., Zody,M.C., Baldwin,J., Devon,K., Dewar,K., Doyle,M., FitzHugh,W. et al. (2001) Initial sequencing and analysis of the human genome. Nature, 409, 860-921.

43. Roberts,L., Davenport,R.J., Pennisi,E. and Marshall,E. (2001) A History of the Human Genome Project. Science, 291, 1195.

44. Venter,J.C., Adams,M.D., Myers,E.W., Li,P.W., Mural,R.J., Sutton,G.G., Smith,H.O., Yandell,M., Evans,C.A., Holt,R.A. et al. (2001) The Sequence of the Human Genome. Science, 291, 1304-1351.

45. Wade,N. Genome of DNA Discover is Deciphered. The New York Times . 6-12007.

Ref Type: Newspaper

46. Zeidlickis,D. Watson Genotype View now On Line - Cold Spring Harbor Laboratory Releases Sequences Browser. Cold Spring Harbor Laboratory News Release . 6-28-2007.

Ref Type: Electronic Citation

47. (2004) The ENCODE (ENCyclopedia Of DNA Elements) Project. Science, 306, 636-640.

48. (2007) Identification and analysis of functional elements in $1 \%$ of the human genome by the ENCODE pilot project. Nature, 447, 799-816.

49. The ENCODE Project: ENCyclopedia Of DNA Elements (http://www.genome.gov/10005107). NHGRI-NIH . 3-11-2008. National Human Genome Research Institute - National Institutes of Health.

Ref Type: Electronic Citation

50. Denoeud,F., Kapranov,P., Ucla,C., Frankish,A., Castelo,R., Drenkow,J., Lagarde,J., Alioto,T., Manzano,C., Chrast,J. et al. (2007) Prominent use of distal 5 ' transcription start sites and discovery of a large number of additional exons in ENCODE regions. Genome Res., 17, 746-759.

51. Gerstein,M.B., Bruce,C., Rozowsky,J.S., Zheng,D., Du,J., Korbel,J.O., Emanuelsson,O., Zhang,Z.D., Weissman,S. and Snyder,M. (2007) What is a gene, post-ENCODE? History and updated definition. Genome Res., 17, 669-681.

52. Rozowsky,J.S., Newburger,D., Sayward,F., Wu,J., Jordan,G., Korbel,J.O., Nagalakshmi,U., Yang,J., Zheng,D., Guigo,R. et al. (2007) The DART classification of unannotated transcription within the ENCODE regions: Associating transcription with known and novel loci. Genome Res., 17, 732-745.

53. Thomas,D.J., Rosenbloom,K.R., Clawson,H., Hinrichs,A.S., Trumbower,H., Raney,B.J., Karolchik,D., Barber,G.P., Harte,R.A., Hillman-Jackson,J. et al. 
(2007) The ENCODE Project at UC Santa Cruz. Nucleic Acids Res., 35, D663D667.

54. Zhang,Z.D., Paccanaro,A., Fu,Y., Weissman,S., Weng,Z., Chang,J., Snyder,M. and Gerstein,M.B. (2007) Statistical analysis of the genomic distribution and correlation of regulatory elements in the ENCODE regions. Genome Res., 17, 787-797.

55. Zheng,D. and Gerstein,M.B. (2006) A computational approach for identifying pseudogenes in the ENCODE regions. Genome Biol., 7 Suppl 1, S13-10.

56. Zheng,D., Frankish,A., Baertsch,R., Kapranov,P., Reymond,A., Choo,S.W., Lu,Y., Denoeud,F., Antonarakis,S.E., Snyder,M. et al. (2007) Pseudogenes in the ENCODE regions: Consensus annotation, analysis of transcription, and evolution. Genome Res., 17, 839-851.

57. (2004) Dictionary of Bioinformatics and Computational Biology. Wiley-Liss, New Jersey.

58. Lewin,B. (1997) Genes. Oxford Univ Pr (Sd).

59. Harwood,J. (2000) The rediscovery of Mendelism in agricultural context: Erich von Tschermak as plant-breeder. C. R. Acad Sci III, 323, 1061-1067.

60. Lenay,C. (2000) Hugo De Vries: from the theory of intracellular pangenesis to the rediscovery of Mendel. C. R. Acad Sci III, 323, 1053-1060.

61. Rheinberger,H.-J. (1995) When Did Carl Correns Read Gregor Mendel's Paper? A Research Note. Isis, 86, 612-616.

62. Allen,G.E. (1978) Thomas Hunt Morgan - The man and his science. Princeton University Press.

63. Sanger,F. and Coulson,A.R. (1975) A rapid method for determining sequences in DNA by primed synthesis with DNA polymerase. J. Mol. Biol., 94, 441-448.

64. Wain,H.M., Lush,M., Ducluzeau,F. and Povey,S. (2002) Genew: the human gene nomenclature database. Nucleic Acids Res., 30, 169-171.

65. Vanin,E.F. (1985) Processed Pseudogenes: Characteristics and Evolution. Annual Reviews Genetics, 1985, 253-272.

66. Gamow,G. (1954) Possible Relation between Deoxyribonucleic Acid and Protein Structures. Nature, 173, 318.

67. Luscombe,N.M., Greenbaum,D. and Gerstein,M. (2001) What is bioinformatics? A proposed definition and overview of the field. Methods Inf. Med, 40, 346-358. 
68. Mizrachi,I. (2007) GenBank: The Nucleotide Sequence Database. pp. 1-1-1-17.

69. Altschul,S.F., Gish,W., Miller,W., Meyers,E.W. and Lipman,D.J. (1990) Basic Local Alignment Search Tool. Journal of Molecular Biology, 215, 403-410.

70. Korf,I., Yandell,M. and Bedell,J. (2003) BLAST-An Essential Guide to the Basic Local Alignment Search Tool. O'Reilly \& Associates, Inc., Sebastopol, CA.

71. Cravedi,K. GenBank Celebrate 25 Years of Service with Two-Days Conference; Leading Scientists Will Discuss the DNA Database at April 7-8 Meeting. NIH . 43-2008.

Ref Type: Electronic Citation

72. Boguski,M.S., Lowe,T.M.J. and Tolstoshev,C.M. (1993) dbEST - database for "expressed sequence tags". Nat Genet, 4, 332-333.

73. Pruitt,K.D., Tatusova,T. and Maglott,D.R. (2007) NCBI reference sequences (RefSeq): a curated non-redundant sequence database of genomes, transcripts and proteins. Nucleic Acids Res., 35, D61-D65.

74. Pruitt,K.D., Katz,K.S., Sicotte,H. and Maglott,D.R. (2000) Introducing RefSeq and LocusLink: curated human genome resources at the NCBI. Trends in Genetics, 16, 44-47.

75. Pruitt,K.D., Tatusova,T. and Maglott,D.R. (2005) NCBI Reference Sequence (RefSeq): a curated non-redundant sequence database of genomes, transcripts and proteins. Nucl. Acids Res., 33, D501-D504.

76. Pruitt,K.D., Tatusova,T. and Maglott,D. (2007) The Reference Sequence (RefSeq) Project. NCBI Handbook. pp. 18-1-18-25.

77. Hinrichs,A.S., Karolchik,D., Baertsch,R., Barber,G.P., Bejerano,G., Clawson,H., Diekhans,M., Furey,T.S., Harte,R.A., Hsu,F. et al. (2006) The UCSC Genome Browser Database: update 2006. Nucleic Acids Res., 34, D590-D598.

78. Karolchik,D., Bejerano,G., Hinrichs,A.S., Kuhn,R.M., Miller,W., Rosenbloom,K.R., Zweig,A.S., Haussler,D. and Kent,W.J. (2007) Comparative genomic analysis using the UCSC genome browser. Methods Mol Biol., 395, 1734.

79. Karolchik,D., Hinrichs,A.S. and Kent,W.J. (2007) The UCSC Genome Browser. Curr Protoc. Bioinformatics, Chapter 1, Unit.

80. Karolchik,D., Kuhn,R.M., Baertsch,R., Barber,G.P., Clawson,H., Diekhans,M., Giardine,B., Harte,R.A., Hinrichs,A.S., Hsu,F. et al. (2008) The UCSC Genome Browser Database: 2008 update. Nucleic Acids Res., 36, D773-D779. 
81. Kuhn,R.M., Karolchik,D., Zweig,A.S., Trumbower,H., Thomas,D.J., Thakkapallayil,A., Sugnet,C.W., Stanke,M., Smith,K.E., Siepel,A. et al. (2007) The UCSC genome browser database: update 2007. Nucleic Acids Res., 35, D668D673.

82. Dubuisson,O. (2000) ASN.1 - Communication between heterogeneous systems. Morgan Kaufmann Publishers.

83. FASTA format description (http://www.ncbi.nlm.nih.gov/blast/fasta.shtml). NCBI . 2004.

Ref Type: Electronic Citation

84. NCBI Data in XML

(http://www.ncbi.nlm.nih.gov/IEB/ToolBox/XML/ncbixml.txt). NCBI . 2000.

Ref Type: Electronic Citation

85. GenBank Flat File Format

(http://www.ncbi.nlm.nih.gov/Sitemap/samplerecord.html). NCBI . 1999.

Ref Type: Electronic Citation

86. Karsch-Mizrachi,I. and Ouellette,B.F.F. (2001) The GenBank Sequence Database. In Baxevanis,A.D. and Ouellette,B.F.F. (eds.), Bioinformatics - A Practical Guide to the Analysis of Genes and Proteins. Wiley-Interscience, pp. 45-63.

87. Bedell,J.A., Korf,I. and Gish,W. (2000) MaskerAid: a performance enhancement to RepeatMasker. Comput. Appl. Biosci., 16, 1040-1041.

88. Smit,A. RepeatMasker documentation (http://ftp.genome.washington.edu/RM/RepeatMasker.html). RepeatMasker . 2003.

Ref Type: Electronic Citation

89. Pevsner,J. (2003) Glossary. Bioinformatics and Functional Genomics. WileyLiss, pp. 717-736.

90. Wootton,J.C. and Federhen,S. (1996) Analysis of compositionally biased regions in sequence databases. Methods Enzymol., 266, 554-571.

91. Dreyfus,S. (2002) Richard Bellman on the Birth of Dynamic Programming. Operations Research, 50, 48-51.

92. Korf,I., Flicek,P., Duan,D. and Brent,M.R. (2001) Integrating genomic homology into gene structure prediction. Comput. Appl. Biosci., 17 Suppl 1, S140-S148.

93. Needleman,S.B. and Wunsch,C.D. (1970) A general method applicable to the search for similarities in the amino acid sequence of two proteins. Journal of Molecular Biology, 48, 443-453. 
94. Smith,T.F. and Waterman,M.S. (1981) Identification of common molecular subsequences. J Mol. Biol., 147, 195-197.

95. Ukkonen,E. On Approximate String Matching. International Foundations of Computationa Theory. Proceedings of the 1983 Foundations of computation thoery , 487-495. 8-21-1983. 8-21-1983.

Ref Type: Conference Proceeding

96. Powell,D.R., Allison,L. and Dix,T.I. (2000) Fast, optimal alignment of three sequences using linear gap costs. J Theor. Biol., 207, 325-336.

97. Pevsner,J. (2003) Pairwise Sequence Alignment. Bioinformatics and Functional Genomics. Wiley-Liss, pp. 41-86.

98. Lipman,D.J. and Pearson,W.R. (1985) Rapid and Sensitive Protein Similarity Search. Science, 227, 1435-1441.

99. Pearson,W.R. and Lipman,D.J. (1988) Improved tools for biological sequence comparison. Proceedings of the National Academy of Sciences of the United States of America (Pro Natl Acad Sci USA), 85, 2444-2448.

100. Pevsner,J. (2003) Bioinformatics and Functional Genomics. Wiley-Liss.

101. Altschul,S.F., Madden,T.L., Schaffer,A.A., Zhang,J., Zhang,Z., Miller,W. and Lipman,D.J. (1997) Gapped BLAST and PSI-BLAST: a new generation of protein database search programs. Nucl. Acids Res., 25, 3389-3402.

102. Zhang,Z., Schaffer,A.A., Miller,W., Madden,T.L., Lipman,D.J., Koonin,E.V. and Altschul,S.F. (1998) Protein sequence similarity searches using patterns as seeds. Nucl. Acids Res., 26, 3986-3990.

103. Gropp,W., Lusk,E. and Skjellum,A. (1999) Using MPI - Portable Parallel Programming with Message Passing Interface. The MIT Press.

104. Gropp,W. and Lusk,E. (2003) Parallel Programming with MPI. In Gropp,W., Lusk,E. and Sterling,T. (eds.), Beowulf Cluster Computing with Linux. Massachusetts Institute of Technology, London, England, pp. 207-244.

105. Darling,A.E., Carey,L. and Feng,W. The Design, Implementation, and Evaluation of mpiBLAST. ClusterWorld Conference \& Expo in conjuction with the 4th International Conference on Linux Clusters: The HPC Revolution 2003. LA-UR 03-2862. 6-23-2003. 2003.

Ref Type: Conference Proceeding

106. The mpiBLAST Team. The mpiBLAST User Guide. http://www.mpiblast.org/Docs/Guide . 2004.

Ref Type: Electronic Citation 
107. Florea,L., Hartzell,G., Zhang,Z., Rubin,G.M. and Miller,W. (1998) A computer program for aligning a cDNA sequence with a genomic DNA sequence. Genome Res., 8, 967-974.

108. Wheelan,S.J., Church,D.M. and Ostell,J.M. (2001) Spidey: a tool for mRNA-togenomic alignments. Genome Res., 11, 1952-1957.

109. Breathnach,R., Benoist,C., O'Hare,K., Gannon,F. and Chambon,P. (1978) Ovalbumin gene: evidence for a leader sequence in mRNA and DNA sequences at the exon-intron boundaries. Proc. Natl Acad Sci U. S. A, 75, 4853-4857.

110. Mount,S.M. (1982) A catalogue of splice junction sequences. Nucl. Acids Res., 10, $459-472$.

111. Mount,S.M. (1983) RNA processing. Sequences that signal where to splice. Nature, 304, 309-310.

112. Rogan,P.K., Faux,B.M. and Schneider,T.D. (1998) Information analysis of human splice site mutations. Hum. Mutat., 12, 153-171.

113. Watt,R., Nishikura,K., Sorrentino,J., ar-Rushdi,A., Croce,C.M. and Rovera,G. (1983) The structure and nucleotide sequence of the 5 ' end of the human c-myc oncogene. Proc. Natl Acad Sci U. S. A, 80, 6307-6311.

114. Mott,R. (1997) EST_GENOME: a program to align spliced DNA sequences to unspliced genomic DNA. Comput. Appl. Biosci., 13, 477-478.

115. Maizel,J.V., Jr. and Lenk,R.P. (1981) Enhanced graphic matrix analysis of nucleic acid and protein sequences. Proc. Natl Acad Sci U. S. A, 78, 7665-7669.

116. Schuler,G.D. (2001) Sequence Alignment and Database Searching. In Baxevanis,A.D. and Ouellette,B.F.F. (eds.), Bioinformatics - A Practical Guide to the Analysis of Genes and Proteins. Wiley-Interscience, pp. 187-214.

117. Wu,T.D. and Watanabe,C.K. (2005) GMAP: a genomic mapping and alignment program for mRNA and EST sequences. Comput. Appl. Biosci., 21, 1859-1875.

118. Zhang,M. and Gish,W. (2006) Improved spliced alignment from an information theoretic approach. Comput. Appl. Biosci., 22, 13-20.

119. Cha,I.E. and Rouchka,E.C. Comparison of Current BLAST Software on Nucleotide Sequences. Proc.19th International Parallel and Distributed Processing Symposium, IPDPS 2005, 4th IEEE International Workshop on High Performance Computational Biology, HiCOMB 2005. Proc.19th International Parallel and Distributed Processing Symposium, IPDPS 2005, 4th IEEE International Workshop on High Performance Computational Biology, HiCOMB 2005 . 2005. IEEE. 4-4-2005.

Ref Type: Conference Proceeding 
120. Ball,B. and Duff,H. (2006) Red Hat Linux 9 Unleashed.

121. (2003) Beowulf Cluster Computing with Linux. Massachusetts Institute of Technology.

122. Davies,J., Whittaker,R. and von Hagen,W. (2005) Suse Linux 9 Bible. John Wiley $\&$ Sons.

123. Roberts,R.J. An Amazing Distortion in DNA Induced by a Methyltransferase. Nobel Lectures, Physiology or Medicine 1991-1995 . 12-8-1993.

Ref Type: In Press

124. Sharp,P.A. (1994) Split genes and RNA splicing. Cell., 77, 805-815.

125. Wall,R., Choi,E., Carter,C., Kuehl,M. and Rogers,J. (1981) RNA processing in immunoglobulin gene expression. Cold Spring Harb. Symp. Quant. Biol., 45 Pt 2:879-85., 879-885.

126. Krainer,A.R., Maniatis,T., Ruskin,B. and Green,M.R. (1984) Normal and mutant human beta-globin pre-mRNAs are faithfully and efficiently spliced in vitro. Cell, 36, 993-1005.

127. Melton,D.A., Krieg,P.A., Rebagliati,M.R., Maniatis,T., Zinn,K. and Green,M.R. (1984) Efficient in vitro synthesis of biologically active RNA and RNA hybridization probes from plasmids containing a bacteriophage SP6 promoter. Nucleic Acids Res., 12, 7035-7056.

128. Ruskin,B., Krainer,A.R., Maniatis,T. and Green,M.R. (1984) Excision of an intact intron as a novel lariat structure during pre-mRNA splicing in vitro. Cell, 38, 317331.

129. Burset,M., Seledtsov,I.A. and Solovyev,V.V. (2000) Analysis of canonical and non-canonical splice sites in mammalian genomes. Nucleic Acids Res., 28, 43644375 .

130. Burset,M., Seledtsov,I.A. and Solovyev,V.V. (2001) SpliceDB: database of canonical and non-canonical mammalian splice sites. Nucleic Acids Res., 29, 255259.

131. Jamison,S.F. and Garcia-Blanco,M.A. (1992) An ATP-independent U2 small nuclear ribonucleoprotein particle/precursor mRNA complex requires both splice sites and the polypyrimidine tract. Proc. Natl. Acad. Sci. U. S. A., 89, 5482-5486.

132. Jamison,S.F., Pasman,Z., Wang,J., Will,C., Luhrmann,R., Manley,J.L. and Garcia-Blanco,M.A. (1995) U1 snRNP-ASF/SF2 interaction and 5' splice site recognition: characterization of required elements. Nucleic Acids Res., 23, 32603267. 
133. Seraphin,B., Kretzner,L. and Rosbash,M. (1988) A U1 snRNA:pre-mRNA base pairing interaction is required early in yeast spliceosome assembly but does not uniquely define the 5' cleavage site. EMBO J., 7, 2533-2538.

134. Gupta,S., Zink,D., Korn,B., Vingron,M. and Haas,S.A. (2004) Genome wide identification and classification of alternative splicing based on EST data. Comput. Appl. Biosci., 20, 2579-2585.

135. Kan,Z., States,D. and Gish,W. (2002) Selecting for functional alternative splices in ESTs. Genome Res., 12, 1837-1845.

136. Sharp,P.A. (2005) The discovery of split genes and RNA splicing. Trends Biochem. Sci., 30, 279-281.

137. Modrek,B. and Lee,C. (2002) A genomic view of alternative splicing. Nat. Genet., 30, 13-19.

138. Sharp,P.A. Split Genes and RNA Splicing. Nobel Lectures, Physiology or Medicine 1991-1995 . 12-8-1993.

Ref Type: In Press

139. Fackenthal,J.D., Cartegni,L., Krainer,A.R. and Olopade,O.I. (2002) BRCA2 T2722R is a deleterious allele that causes exon skipping. Am. J Hum. Genet, 71, $625-631$.

140. Chen,F.C., Chen,C.J., Ho,J.Y. and Chuang,T.J. (2006) Identification and evolutionary analysis of novel exons and alternative splicing events using crossspecies EST-to-genome comparisons in human, mouse and rat. $B M C$ Bioinformatics, 7, 136.

141. Brett,D., Hanke,J., Lehmann,G., Haase,S., Delbruck,S., Krueger,S., Reich,J. and Bork,P. (2000) EST comparison indicates $38 \%$ of human mRNAs contain possible alternative splice forms. FEBS Lett., 474, 83-86.

142. Mironov,A.A., Fickett,J.W. and Gelfand,M.S. (1999) Frequent alternative splicing of human genes. Genome Res., 9, 1288-1293.

143. Blaustein,M., Pelisch,F. and Srebrow,A. (2007) Signals, pathways and splicing regulation. Int. J. Biochem. Cell Biol., 39, 2031-2048.

144. Zipursky,S.L., Wojtowicz,W.M. and Hattori,D. (2006) Got diversity? Wiring the fly brain with Dscam. Trends Biochem. Sci., 31, 581-588.

145. Kidd,K.K., Pakstis,A.J., Speed,W.C. and Kidd,J.R. (2004) Understanding human DNA sequence variation. J. Hered., 95, 406-420. 
146. Kan,Z., Rouchka,E.C., Gish,W.R. and States,D.J. (2001) Gene Structure Prediction and Alternative Splicing Analysis Using Genomically Aligned ESTs. Genome Res., 11, 889-900.

147. Johnson,J.M., Castle,J., Garrett-Engele,P., Kan,Z., Loerch,P.M., Armour,C.D., Santos,R., Schadt,E.E., Stoughton,R. and Shoemaker,D.D. (2003) Genome-wide survey of human alternative pre-mRNA splicing with exon junction microarrays. Science., 302, 2141-2144.

148. Kwan,T., Benovoy,D., Dias,C., Gurd,S., Provencher,C., Beaulieu,P., Hudson,T.J., Sladek,R. and Majewski,J. (2008) Genome-wide analysis of transcript isoform variation in humans. Nat Genet, 40, 225-231.

149. Mockler,T.C., Chan,S., Sundaresan,A., Chen,H., Jacobsen,S.E. and Ecker,J.R. (2005) Applications of DNA tiling arrays for whole-genome analysis. Genomics, 85, 1-15.

150. Hiller,M., Backofen,R., Heymann,S., Busch,A., Glaesser,T.M. and Freytag,J.C. (2004) Efficient prediction of alternative splice forms using protein domain homology. In Silico. Biol., 4, 195-208.

151. Florea,L., Di,F., V, Miller,J., Turner,R., Yao,A., Harris,M., Walenz,B., Mobarry,C., Merkulov,G.V., Charlab,R. et al. (2005) Gene and alternative splicing annotation with AIR. Genome Res., 15, 54-66.

152. Croft,L., Schandorff,S., Clark,F., Burrage,K., Arctander,P. and Mattick,J.S. (2000) ISIS, the intron information system, reveals the high frequency of alternative splicing in the human genome. Nat Genet, 24, 340-341.

153. Modrek,B., Resch,A., Grasso,C. and Lee,C. (2001) Genome-wide detection of alternative splicing in expressed sequences of human genes. Nucleic Acids Res., 29, 2850-2859.

154. Lee,C., Atanelov,L., Modrek,B. and Xing,Y. (2003) ASAP: the Alternative Splicing Annotation Project. Nucleic Acids Res., 31, 101-105.

155. Sorek,R., Shamir,R. and Ast,G. (2004) How prevalent is functional alternative splicing in the human genome? Trends Genet., 20, 68-71.

156. Castrignano,T., D'Antonio,M., Anselmo,A., Carrabino,D., Onorio De,M.A., D'Erchia,A.M., Licciulli,F., Mangiulli,M., Mignone,F., Pavesi,G. et al. (2008) ASPicDB: a database resource for alternative splicing analysis. Comput. Appl. Biosci., 24, 1300-1304.

157. Dousa,T.P. (1999) Cyclic-3',5'-nucleotide phosphodiesterase isozymes in cell biology and pathophysiology of the kidney. Kidney Int., 55, 29-62. 
158. Bolger,G.B. (1994) Molecular biology of the cyclic AMP-specific cyclic nucleotide phosphodiesterases: a diverse family of regulatory enzymes. Cell Signal., 6, 851-859.

159. Marchmont,R.J. and Houslay,M.D. (1980) A peripheral and an intrinsic enzyme constitute the cyclic AMP phosphodiesterase activity of rat liver plasma membranes. Biochem. J., 187, 381-392.

160. Wachtel,H. (1982) Characteristic behavioural alterations in rats induced by rolipram and other selective adenosine cyclic 3', 5'-monophosphate phosphodiesterase inhibitors. Psychopharmacology (Berl)., 77, 309-316.

161. Doherty,A.M. (1999) Phosphodiesterase 4 inhibitors as novel anti-inflammatory agents. Curr. Opin. Chem. Biol., 3, 466-473.

162. Boswell-Smith,V., Spina,D. and Page,C.P. (2006) Phosphodiesterase inhibitors. Br. J. Pharmacol., 147 Suppl 1:S252-7., S252-S257.

163. Lugnier,C. (2006) Cyclic nucleotide phosphodiesterase (PDE) superfamily: a new target for the development of specific therapeutic agents. Pharmacol Ther., 109, 366-398.

164. Pearse,D.D., Pereira,F.C., Marcillo,A.E., Bates,M.L., Berrocal,Y.A., Filbin,M.T. and Bunge,M.B. (2004) cAMP and Schwann cells promote axonal growth and functional recovery after spinal cord injury. Nat Med, 10, 610-616.

165. Song,Q., Cole,J.W., O'Connell,J.R., Stine,O.C., Gallagher,M., Giles,W.H., Mitchell,B.D., Wozniak,M.A., Stern,B.J., Sorkin,J.D. et al. (2006) Phosphodiesterase 4D polymorphisms and the risk of cerebral infarction in a biracial population: the Stroke Prevention in Young Women Study. Hum. Mol Genet., 15, 2468-2478.

166. Houslay,M.D., Baillie,G.S. and Maurice,D.H. (2007) cAMP-Specific phosphodiesterase-4 enzymes in the cardiovascular system: a molecular toolbox for generating compartmentalized cAMP signaling. Circ. Res., 100, 950-966.

167. Gish,W. 1996-2006 Personal Communication to Author's Advisor. 2006. Ref Type: Unpublished Work

168. Kent,W.J. (2002) BLAT---The BLAST-Like Alignment Tool. Genome Res., 12, 656-664.

169. Wheeler,D.L., Barrett,T., Benson,D.A., Bryant,S.H., Canese,K., Church,D.M., DiCuccio,M., Edgar,R., Federhen,S., Helmberg,W. et al. (2005) Database resources of the National Center for Biotechnology Information. Nucleic Acids Res., 33, D39-D45. 
170. Larkin,M.A., Blackshields,G., Brown,N.P., Chenna,R., McGettigan,P.A., McWilliam,H., Valentin,F., Wallace,I.M., Wilm,A., Lopez,R. et al. (2007) Clustal W and Clustal X version 2.0. Bioinformatics., 23, 2947-2948.

171. Saiki,R.K., Scharf,S., Faloona,F., Mullis,K.B., Horn,G.T., Erlich,H.A. and Arnheim,N. (1985) Enzymatic amplification of beta-globin genomic sequences and restriction site analysis for diagnosis of sickle cell anemia. Science, 230, $1350-1354$.

172. Saiki,R.K., Gelfand,D.H., Stoffel,S., Scharf,S.J., Higuchi,R., Horn,G.T., Mullis,K.B. and Erlich,H.A. (1988) Primer-directed enzymatic amplification of DNA with a thermostable DNA polymerase. Science, 239, 487-491.

173. Joens,N.L. (2002) PCR: Principles, Procedures, and Parameters. In Theophilus,B.D.M. and Rapley,R. (eds.), PCR Mutation Detection Protocols. Humana Press Inc., pp. 37-46.

174. Mullis,K.B. and Faloona,F.A. (1987) Specific synthesis of DNA in vitro via a polymerase-catalyzed chain reaction. Methods Enzymol., 155, 335-350.

175. Rouchka,E.C., Khalyfa,A. and Cooper,N.G. (2005) MPrime: efficient large scale multiple primer and oligonucleotide design for customized gene microarrays. BMC. Bioinformatics., 6:175., 175.

176. Cooper,D.N. (1999) Pseudogenes and their formation. Human Gene Evolution. BIOS Scientific Publishers, Oxford, UK, pp. 265-285.

177. Dunham,I., Shimizu,N., Roe,B.A., Chissoe,S., Hunt,A.R., Collins,J.E., Bruskiewich,R., Beare,D.M., Clamp,M., Smink,L.J. et al. (1999) The DNA sequence of human chromosome 22. Nature, 402, 489-495.

178. Gibson,L.J. (1994) Pseudogenes and Origins. Origins, 21, 91-108.

179. Zhang,Z., Harrison,P.M., Liu,Y. and Gerstein,M. (2003) Millions of years of evolution preserved: a comprehensive catalog of the processed pseudogenes in the human genome. Genome Res., 13, 2541-2558.

180. Torrents,D., Suyama,M., Zdobnov,E. and Bork,P. (2003) A Genome-Wide Survey of Human Pseudogenes. Genome Res., 13, 2559-2567.

181. Ohshima,K., Hattori,M., Yada,T., Gojobori,T., Sakaki,Y. and Okada,N. (2003) Whole-genome screening indicates a possible burst of formation of processed pseudogenes and Alu repeats by particular L1 subfamilies in ancestral primates. Genome Biol., 4, R74.

182. Zhang,Z. and Gerstein,M. (2004) Large-scale Analysis of Pseudogenes in the Human Genome. Curr Opin Genet Dev, 14, 328-335. 
183. van Baren,M.J. and Brent,M.R. (2006) Iterative gene prediction and pseudogene removal improves genome annotation. Genome Res., 16, 678-685.

184. Mighell,A.J., Smith,N.R., Robinson,P.A. and Markham,A.F. (2000) Vertebrate pseudogenes. FEBS Letters, 468, 109-114.

185. Gerstein,M. and Zheng,D. (2006) The real life of pseudogenes. Sci Am., 295, 4855.

186. Nishikimi,M., Kawai,T. and Yagi,K. (1992) Guinea pigs possess a highly mutated gene for L-gulono-gamma-lactone oxidase, the key enzyme for L-ascorbic acid biosynthesis missing in this species. J Biol. Chem., 267, 21967-21972.

187. Nishikimi,M., Fukuyama,R., Minoshima,S., Shimizu,N. and Yagi,K. (1994) Cloning and chromosomal mapping of the human nonfunctional gene for Lgulono-gamma-lactone oxidase, the enzyme for L-ascorbic acid biosynthesis missing in man. J Biol. Chem., 269, 13685-13688.

188. Xue,Y., Daly,A., Yngvadottir,B., Liu,M., Coop,G., Kim,Y., Sabeti,P., Chen,Y., Stalker,J., Huckle,E. et al. (2006) Spread of an inactive form of caspase-12 in humans is due to recent positive selection. Am. J Hum. Genet, 78, 659-670.

189. Hirotsune,S., Yoshida,N., Chen,A., Garrett,L., Sugiyama,F., Takahashi,S., Yagami,K., Wynshaw-Boris,A. and Yoshiki,A. (2003) An expressed pseudogene regulates the messenger-RNA stability of its homologous coding gene. Nature, 423, 91-96.

190. Hirotsune,S. (2003) An expressed pseudogene regulates mRNA stability of its homologous coding gene. PNE, 48, 1908-1912.

191. Yano,Y., Saito,R., Yoshida,N., Yoshiki,A., Wynshaw-Boris,A., Tomita,M. and Hirotsune,S. (2004) A new role for expressed pseudogenes as ncRNA: regulation of mRNA stability of its homologous coding gene. J Mol Med, 82, 414-422.

192. Gray,T.A., Wilson,A., Fortin,P.J. and Nicholls,R.D. (2006) The putatively functional Mkrn1-p1 pseudogene is neither expressed nor imprinted, nor does it regulate its source gene in trans. Proc. Natl Acad Sci U. S. A, 103, 12039-12044.

193. Harrison,P.M., Echols,N. and Gerstein,M.B. (2001) Digging for dead genes: an analysis of the characteristics of the pseudogene population in the Caenorhabditis elegans genome. Nucleic Acids Res., 29, 818-830.

194. Harrison,P.M., Hegyi,H., Balasubramanian,S., Luscombe,N.M., Bertone,P., Echols,N., Johnson,T. and Gerstein,M. (2002) Molecular Fossils in the Human Genome: Identification and Analysis of the Pseudogenes in Chromosomes 21 and 22. Genome Res., 12, 272-280. 
195. Sakai,H., Koyanagi,K.O., Itoh,T., Imanishi,T. and Gojobori,T. (2003) Detection of Processed Pseudogenes Based on cDNA Mapping to the Human Genome. Genome Informatics, 14, 452-453.

196. Zhang,Z., Carriero,N., Zheng,D., Karro,J., Harrison,P.M. and Gerstein,M. (2006) PseudoPipe: an automated pseudogene identification pipeline. Comput. Appl. Biosci., 22, 1437-1439.

197. Zhang,Z., Harrison,P. and Gerstein,M. (2002) Identification and analysis of over 2000 ribosomal protein pseudogenes in the human genome. Genome Res., 12, 1466-1482.

198. Chiu,K.P., Ariyaratne,P., Xu,H., Tan,A., Ng,P., Liu,E.T., Ruan,Y., Wei,C.L. and Sung,W.K. (2007) Pathway aberrations of murine melanoma cells observed in Paired-End diTag transcriptomes. BMC Cancer, 7, 109.

199. Harrow,J., Denoeud,F., Frankish,A., Reymond,A., Chen,C.K., Chrast,J., Lagarde,J., Gilbert,J.G., Storey,R., Swarbreck,D. et al. (2006) GENCODE: producing a reference annotation for ENCODE. Genome Biol., 7 Suppl 1, S4-S9.

200. Kent,W.J., Baertsch,R., Hinrichs,A., Miller,W. and Haussler,D. (2003) Evolution's cauldron: duplication, deletion, and rearrangement in the mouse and human genomes. Proc. Natl Acad Sci U. S. A, 100, 11484-11489.

201. Schwartz,S., Kent,W.J., Smit,A., Zhang,Z., Baertsch,R., Hardison,R.C., Haussler,D. and Miller,W. (2003) Human-mouse alignments with BLASTZ. Genome Res., 13, 103-107.

202. Bairoch,A., Apweiler,R., Wu,C.H., Barker,W.C., Boeckmann,B., Ferro,S., Gasteiger,E., Huang,H., Lopez,R., Magrane,M. et al. (2005) The Universal Protein Resource (UniProt). Nucleic Acids Res., 33, D154-D159.

203. Elzanowski,A. and Ostell,J. The Genetic Codes (http://www.ncbi.nlm.nih.gov/Taxonomy/Utils/wprintgc.cgi). NCBI Taxonomy Browser . 2007.

Ref Type: Electronic Citation

204. Abramczyk,D., Tchorzewski,M. and Grankowski,N. (2003) Non-AUG translation initiation of mRNA encoding acidic ribosomal P2A protein in Candida albicans. Yeast, 20, 1045-1052.

205. Hann,S.R., King,M.W., Bentley,D.L., Anderson,C.W. and Eisenman,R.N. (1988) A non-AUG translational initiation in c-myc exon 1 generates an $\mathrm{N}$-terminally distinct protein whose synthesis is disrupted in Burkitt's lymphomas. Cell, 52, 185-195.

206. Peabody,D.S. (1989) Translation initiation at non-AUG triplets in mammalian cells. J Biol. Chem., 264, 5031-5035. 
207. Prats,H., Kaghad,M., Prats,A.C., Klagsbrun,M., Lelias,J.M., Liauzun,P., Chalon,P., Tauber,J.P., Amalric,F., Smith,J.A. et al. (1989) High molecular mass forms of basic fibroblast growth factor are initiated by alternative CUG codons. Proc. Natl Acad Sci U. S. A, 86, 1836-1840.

208. Sugihara,H., Andrisani,V. and Salvaterra,P.M. (1990) Drosophila choline acetyltransferase uses a non-AUG initiation codon and full length RNA is inefficiently translated. J Biol. Chem., 265, 21714-21719.

209. Takahashi,K., Maruyama,M., Tokuzawa,Y., Murakami,M., Oda,Y., Yoshikane,N., Makabe,K.W., Ichisaka,T. and Yamanaka,S. (2005) Evolutionarily conserved non-AUG translation initiation in NAT1/p97/DAP5 (EIF4G2). Genomics, 85, 360-371.

210. Brenner,S., Stretton,A.O. and Kaplan,S. (1965) Genetic code: the 'nonsense' triplets for chain termination and their suppression. Nature, 206, 994-998.

211. Brenner,S., Barnett,L., Katz,E.R. and Crick,F.H. (1967) UGA: a third nonsense triplet in the genetic code. Nature, 213, 449-450.

212. Sambrook,J.F., Fan,D.P. and Brenner,S. (1967) A strong suppressor specific for UGA. Nature, 214, 452-453.

213. Tian,B., Hu,J., Zhang,H. and Lutz,C.S. (2005) A large-scale analysis of mRNA polyadenylation of human and mouse genes. Nucleic Acids Res., 33, 201-212.

214. Beaudoing,E., Freier,S., Wyatt,J.R., Claverie,J.M. and Gautheret,D. (2000) Patterns of variant polyadenylation signal usage in human genes. Genome Res., 10, 1001-1010.

215. Harrison,P.M., Milburn,D., Zhang,Z., Bertone,P. and Gerstein,M. (2003) Identification of pseudogenes in the Drosophila melanogaster genome. Nucleic Acids Res., 31, 1033-1037.

216. Zhang,Z. and Gerstein,M. (2003) Patterns of nucleotide substitution, insertion and deletion in the human genome inferred from pseudogenes. Nucleic Acids Res., 31, 5338-5348.

217. Zhang,Z., Carriero,N. and Gerstein,M. (2004) Comparative analysis of processed pseudogenes in the mouse and human genomes. Trends Genet, 20, 62-67.

218. Graur,D. and Li,W.-H. (1999) Evolutionary Change in Nucleotide Sequences. In Sinauer,A.D. and Wigg,C.J. (eds.), Fundamentals of Molecular Evolution. Sinauer Associates, Inc., Sunderland, MA 01375, pp. 67-98.

219. Eddy,S.R. (2004) Where did the BLOSUM62 alignment score matrix come from? Nat Biotechnol., 22, 1035-1036. 
220. Henikoff,S. and Henikoff,J.G. (1992) Amino acid substitution matrices from protein blocks. Proc. Natl Acad Sci U. S. A, 89, 10915-10919.

221. Slater,G.S.C. (1998) Human EST Sequences. In Bishop,M.J. (ed.), Guide to Human Genome Computing. Academic Press, pp. 205-214.

222. Dale,J.W. and von Schantz,M. (2002) From Genes to Genomes: Concepts and Applications of DNA Technology. John Wiley \& Sons.

223. Zhang,Z., Schwartz,S., Wagner,L. and Miller,W. (2000) A greedy algorithm for aligning DNA sequences. J. Comput. Biol., 7, 203-214.

224. Costa,R.L.d.C. and Lifschitz,S. (2003) Database Allocation Strategies for Parallel BLAST Evaluation on Clusters. Distributed and Parallel Databases, 13, 99-127.

225. Mathog,D.R. (2003) Parallel BLAST on split databases. Comput. Appl. Biosci., 19, 1865-1866.

226. Osorio,E.C., de Souza,J.E., Zaiats,A.C., de Oliveira,P.S. and de Souza,S.J. (2003) pp-Blast: a "pseudo-parallel" Blast. Braz. J Med Biol. Res., 36, 463-464.

227. Rangwala,H., Lantz,E., Musselman,R., Pinnow,K., Smith,B. and Wallenfelt,B. Massively Parallel BLAST for the Blue Gene/L. High Availability and Performance Computing Workshop 2005. 10-11-2005.

Ref Type: Conference Proceeding 


\section{APPENDIX I}

\section{ABBREVIATIONS}

ASN.1 - abstract syntax notation one

BLOSUM - blocks of amino acid substitution matrix

bp - base pair

cAMP - cyclic adenosine monophosphate

cDNA - complementary DNA

CDS - coding sequence

cGMP - cyclic guanosine monophosphate

CNS - central nervous system

COPD - chronic obstructive pulmonary disease

CSHL - Cold Spring Harbor Laboratory

CPU - central process unit

dbEST - expressed sequence tags database

DDBJ - DNA DataBank of Japan

ddNTP - dideoxynucleotide triphosphate

DNA - deoxyribonucleic acid

ELSI - ethical, legal, and social issues

EMBL - European Molecular Biology Laboratory

ENCODE - ENCyclopedia of DNA Elements

EST - expressed sequence tag

GI - GenInfo Identifier

GMAP - genomic mapping and alignment program

HGP - Human Genome Project

HSP - high-scoring pair

INSDC - International Nucleotide Sequence Database Collaboration

IUB - International Union of Biochemistry

IUPAC - International Union of Pure and Applied Chemistry

MPI - message passing interface

mRNA - messenger RNA

miRNA - microRNA

NAS - National Academy of Science

NCBI - National Center for Biotechnology Information 
NIH - National Institutes of Health

NLM - National Library of Medicine

$\mathrm{ORF}$ - open reading frame

PAM - point accepted mutation

PBS - portable batch system

PCR - polymerase chain reaction

PDE - phosphodiesterase

PDE4 - phosphodiesterase 4

PPi - pyrophosphate

PSSM - position specific scoring matrix

RACE - rapid amplification of cDNA ends

RefSeq - Reference Sequence

RNA - ribonucleic acid

RNAi - RNA interference

rRNA - ribosomal RNA

SARS - severe acute respiratory syndrome

siRNA - silencing RNA, short interfering RNA or small interfering RNA

snRNA - small nuclear RNA

tRNA - transfer RNA

TIGR - The Institute for Genomic Research

UCSC - University of California Santa Cruz

UCR - upstream conserved region

UTR - untranslated region

WU - Washington University at Saint Louis

$\mathrm{XML}$ - extensible markup language 


\section{APPENDIX II}

\section{GLOSSARY}

3' and 5'- Directionality in molecular biology refers to the end-to-end chemical orientation of a single strand of nucleic acid. The chemical convention of naming carbon atoms in the nucleotide sugar-ring numerically gives rise to a 5 , end and a 3' end (usually pronounce "five prime end" and "three prime end"). Therefore, the 5' end designates the end of the DNA or RNA strand that has the fifth carbon in the sugar-ring of the (deoxy)ribose at its terminus. The 3' end has the third carbon in the sugar-ring as its terminus (Figure 1.1).

$\boldsymbol{a b}$ initio - In computation, $a b$ initio means an extra, transform, load tool used to manipulate data. In Bioinformatics, $a b$ initio is a term used to define methods for making predictions about biological features using only a computational model without extrinsic comparison to existing data. In this context, it may be sometimes interchangeable with the Latin term de novo.

alternative splicing - process of mRNA transcription through which the coding regions for a eukaryotic gene, called exons, are extracting and connected to produce alternative mRNA molecules.

amino acid - the basic elements of proteins. Amino acids form short polymer chains called peptides or longer sequences called polypeptides. 
BLAST - stands for basic local alignment search tool which is widely used in Bioinformatics and Computational Biology to find out the basic alignment results between two sequences.

BLOSUM - stands for blocks of amino acid substitution matrix. It is a substitution matrix used for sequence alignment of proteins. It was generated by Henikoff and Henikoff in 1992. The most commonly used matrix is BLOSUM62.

cDNA - stands for complementary DNA. It is a single-stranded DNA made in a laboratory so that its base sequence is complementary to a messenger RNA template. It is assembled by the enzyme reverse transcriptase and may be used in gene cloning or as a gene probe.

central dogma - a process to derive a genetic DNA sequence to a fully-functional protein that contains transcription, pre-mRNA processing, translation and protein folding.

cleavage - a series of mitotic cell divisions that produces a blastula from a fertilized ovum. It is the basis of the multi-cellularity of complex organisms. It can be called segmentation as well.

cleavage site - a DNA sequence that can be recognized and cut by a specific restriction enzyme.

codon - a sequence of three adjacent nucleotides constituting the genetic code that determines the insertion of a specific amino acid in a polypeptide chain during protein synthesis or the signal to stop protein synthesis. 
contig - a continuous sequence of DNA created by assembly overlapping sequenced fragments of a chromosome. It is also a group of clones representing overlapping regions of a genome.

CpG islands - short genomic regions that contain a high frequency of CG dinucleotides than in other regions. ' $p$ ' indicates that ' $C$ ' and ' $G$ ' are connected by phosphodiesterase bond.

cytochrome - a class of hemoprotein. The major biological function is for electron transport by virtue of a reversible valency change of the heme iron.

dbEST -a division of GenBank that collects ESTs submitted from around the world. It contains sequence data and its annotation information from numerous organisms.

ddNTP - stands for dideoxynucleotide, represents one of the four portions: ddATP, ddCTP, ddGTP and ddTTP.

de novo - anew, afresh, the same as if it had not been heard before and as if no decision previously had been rendered.

DNA - stands for deoxyribonucleic acid. It carries the genetic information in the cell and is capable of self-replication and synthesis of RNA. It has four bases -adenine $(A)$, cytosine $(C)$, guanine $(\mathrm{G})$ and thymine $(T)$. DNA consists of two long chains of nucleotides twisted into a double helix and joined by hydrogen bonds between the complementary bases $\mathrm{A}$ to $\mathrm{T}$ or $\mathrm{C}$ to $\mathrm{G}$. The sequence of nucleotides determines individual hereditary characteristics.

DNA sequencing - a method to determine the order of the nucleotide bases, A, C, G and $\mathrm{T}$, in a DNA oligonucleotide. 
DUST - is a computational program used for masking or filtering low complexity regions in nucleic acids.

dynamic programming - a method of solving problems when the solutions can be determined recursively by building upon known solutions of a subspace.

electrophoresis - a method of separating substances, especially proteins, and analyzing molecular structure based on the rate of movement of each component in colloidal suspension while under the influence of an electric field. This method was introduced by Arne Tiselius in 1937 for separating proteins in solution.

ENCODE - stands for encyclopedia of DNA elements. It is an international-wide research to identify the functional elements in the human genome. ENCODE project contains three phase, a pilot phase, a technology development phase and a production phase.

EST - stands for expressed sequence tag, which is a segment of cDNA. The size ranges from 300 to 600 base pair. They are used to identify gene transcripts and are instrumental in gene discovery and gene sequence determination.

exon - is coding region in the genomic sequence. It is a discontinuous sequence of DNA that codes for protein synthesis and carries the genetic code for the final messenger RNA molecule.

e-value - is the sum of all possible values for a random variable, each value multiplied by its probability of occurrence. It is also the integral of the probability density function and a continuous random variable over its range of values.

FASTA - stands for FAST-ALL. It was developed by Pearson and Lipman. The method combines local search with a heuristic algorithm to search a database for sequence 
homology. FASTA compares protein or nucleotide sequences to report the best matches of sequences for local alignment.

FASTA format - the data format begins with a greater-than symbol '>', followed by single line description. The next few lines are sequence data, the combination of A, C, G and T nucleic acids or twenty different amino acids.

frame shifting - a genetic mutation that inserts or deletes a number of nucleotides that is not evenly divisible by three.

GC\% - the percentage of total number of G's and C's in the sequence.

GenBank - is a sequence data bank which was established in 1982 by the National Institutes of Health to help advance scientific discovery worldwide. GenBank collects DNA and RNA sequences and exchanges data to European Molecular Biology Laboratory and the DNA Data Bank of Japan daily.

GenBank format - the data format contains complete sequence information including locus, definition, accession, version, keywords, source, reference, comment, features, base count, and origin.

gene - the basic unit to transfer hereditary information in genome. Genes provide structure, function and regulation to a biological system.

GI - stands for GenInfo Identifier which is used as a unique sequence identifier for nucleotide and proteins. The GI number has been used by NCBI to track sequence histories in GenBank and the other sequences databases it maintains. The VERSION system of identifiers was adopted in 1999 by the International Nucleotide Sequence Database Collaboration (GenBank, EMBL and DDBJ). 
genome annotation - a process of assigning biological information to sequences. The process is based on two main steps: identifying elements on the genome and adding the biological information to the elements. An annotation is a note added by way of commentary and explanation. Once a genome is sequenced, it needs to be annotated and clarified.

global alignment - is aligning the entire length of two sequences to find out sequence similarity.

Hamming distance - if two sequences of length $N$ differ from each other at $n$ sites, then the proportion of differences, $n / N$, is referred to as the Hamming distance or the degree of divergence.

heuristic algorithm - an algorithm that can make an approximation of the best solution without exhaustively considering every possible outcome. It tries to reduce time and space complexity in calculation.

high-throughput sequencing - a fast method of determining the order of bases in DNA.

homology - similar characteristics in two animals that are a product of descent from a common ancestor rather than a product of a similar environment.

Human Genome Project - a project was to sequence the human genome. The project began in 1990 to determine genes in the human genome. In 2001, the first draft was published. In 2003, 95\% of the gene-containing part of the human sequence was mapped to $99.99 \%$ accuracy.

in silico - an expression used to mean performing on computer or via computer simulation. 
in situ hybridization - a technique in which single-stranded DNA are permitted to interact so that hybrids are formed by molecules with sufficiently similar, complementary sequences. It involves hybridizing a labeled nucleic acid in fluorescent dye to suitably prepared cells or histological sections. In situ hybridization is used particularly to look for specific transcription or localization of genes to specific chromosomes.

in vitro - a test performing in the glass or plastic vessels in the laboratory intron - non-coding region in the genomic sequence. Introns are sections of DNA that are not expressed in the gene product.

isoform - a version of protein with only small differences to another isoform of the same protein. Different forms of a protein may be produced from different by related genes. It may also arise from the same gene by alternative splicing.

IUB - stands for International Union of Biochemistry. It provides recommendations on biochemical and organic nomenclature, symbols and terminology.

IUPAC - stands for International Union of Pure and Applied Chemistry. It serves to advance the worldwide aspects of the chemical sciences and to contribute to the application of chemistry in the service of Mankind.

local alignment - an algorithmic technique that looks for significant similarities in smaller sections of the sequences.

locus - the position that a given gene occupies on a chromosome .

low complexity region - the region of biased composition including homopolymeric runs, short-period repeats, and more subtle overrepresentation of one or a few residues. 
MALDI-TOF-MS - stands for matrix-assisted laser desorption ionization time-of-flight mass spectrometry.

MPI - stands for message passing interface which is a library specification for message passing. It is a standard application programming interface (API) that can be used to create parallel applications.

mpiBLAST - a parallel implementation using message passing interface to conduct NCBI BLAST in multi-processor cluster.

mRNA - stands for messenger RNA. It is the form of RNA that mediates the transfer of genetic information from the cell nucleus to ribosomes in the cytoplasm, where it serves as a template for protein synthesis. mRNA is synthesized from a DNA template during the process of transcription.

miRNA - stands for microRNA which are single-stranded RNA molecules about 21-23 nucleotides in length and regulates gene expression.

mutation - a change of the DNA sequence within a gene or chromosome of an organism resulting in the creation of a new character or trait not found in the parental type. Mutation can also mean the process by which such a change occurs in a chromosome, either through an alternation in the nucleotide sequence of the DNA coding for a gene or through a change in the physical arrangement of a chromosome. 
NCBI - stands for National Center for Biotechnology Information. It is established in 1988 as a national resource for molecular biology information. NCBI creates public databases, conducts research in computational biology, develops software tools for analyzing genome data and disseminates biomedical information - all for the better understanding of molecular processes affecting human health and disease.

NIH - stands for National Institute of Health, which is the nation's medical research agency.NIH provides resources and financial support for scholars to conduct researches in medical related science.

nonsynonymous mutation - a mutation that occurs in the nucleotide level but affects the amino acid in translation.

oligonucleotide (or oligo) - a short polymer segment of RNA or DNA, the length ranges from two to twenty bases.

orthologous locus - genes that have evolved directly from an ancestral gene.

PAM - shorts for point accepted mutation. It is a matrix of score to consider the replacement of one amino acid in a protein by another residue that has been accepted by natural selection. It was released by Dayhoff and her colleagues in 1970s. The common used matrix for amino acid sequence comparison is PAM250.

PBS - stands for portable batch system. It is a scheduling system to conduct processes running on multi-processors in the Unix/Linux system. PBS provides additional controls over initiating or scheduling execution of batch jobs and allows routing of those jobs between different hosts. 
PCR - stands for polymerase chain reaction. It is a method to amplify specific DNA segments by exploiting certain features of DNA replication in vitro. The PCR technique was developed by Kary Mullis in 1983. This technique uses a pair of primers, which are DNA segments approximately $20 \mathrm{bp}$ long complement the DNA being synthesized and a thermo stable DNA polymerase to achieve nearexponential enzymatic amplification of target DNA.

PDE - stands for phosphodiesterase. PDEs are enzymes which act as regulators for signal transduction. Currently, there are eleven known families of PDEs. PDEs degrade cyclic adenosine monophosphate (cAMP) and cyclic guanosine monophosphate (cGMP).

PDE4 - stands for phosphodiesterase 4 which is an enzyme being targeted for inflammatory disorders, including asthma and chronic obstructive pulmonary disease and central nervous system injury. PDE4 has a large number of potential isoforms with apparent tissue and functional specificity.

Pfam - Pfam (http://pfam.sanger.ac.uk) is a large collection of multiple sequence alignments and hidden Markov models covering many common protein domains and families. For each Pfam family, users are available to check for multiple alignments, view protein domain architectures, examines species distribution, connect to other databases, and study known protein structures.

polyadenyl (polyA) signal - a six-base sequence which appears in the 3' UTR. AATAAA is the most found pattern in mammalian genomes. 
polyadenyl (polyA) tail - a sequence of 50 to 250 adenine (A) residues that are added to the 3' end of an mRNA sequence after being cleaved. This reaction is catalyzed by polyadenylate polymerase.

polymerase - a polymerase is an enzyme that can synthesize new DNA strands using a DNA template while several such enzymes exist.

primer - a segment of DNA or RNA that is complementary to a given DNA sequence and that is needed to initiate replication by DNA polymerase.

probe - a single-stranded DNA molecule used in laboratory experiments to detect the presence of complementary sequence among a mixture of other single-stranded DNA molecules.

promoter - a sequence of DNA that specifies the sites of transcription initiation for an RNA polymerase.

protein - a linear polymer built from amino acids. Proteins carry out all of a cell's activities.

pseudogene - a genomic sequence that looks like gene but does not have any functionality as gene. Currently there are three known types of pseudogenes: processed pseudogene, unprocessed pseudogene and disable pseudogene.

RACE - short for rapid amplification of cDNA ends, is a technique used in molecular biology to obtain the partial sequence of an RNA transcript found within a cell. RACE begins through RT-PCR to produce a cDNA copy of a region of the RNA transcript. An unknown end portion of a transcript is copied using a known sequence from the center of the transcript. The copied region is bounded by the known sequence, and either the 5' or 3' end. 
RefSeq - Reference Sequence database is a collection of annotated DNA, RNA and protein sequences from major organisms, including plasmids, organelles, viruses, archaea, bacteria and eukaryotes. RefSeq contains data extracted from GenBank and involves varying levels of computational and experimental verification from authoritative groups.

repetitive element - a sequence that appears more than once in a genome.

RepeatMasker - a software package designing to screen DNA sequences for repeats.

retrotransposition - a process occurring in the intermediate RNA stage that a transposon is created by reverse transcription of an RNA molecule.

ribosome - a minute round particle composed of RNA and protein. It is found in the cytoplasm of living cells and serves as the site of assembly by polypeptides encoded by messenger RNA.

RNA - stands for ribonucleic acid. It is a polymeric constituent of all living cells and many viruses. RNA consists of a long and single-stranded chain of alternative phosphate and ribose units with the bases adenine (A), cytosine (C), guanine $(\mathrm{G})$, and uracil $(\mathrm{U})$ bonded to the ribose. The structure and bases sequence of RNA are determinants of protein synthesis and the transmission of genetic information.

RNAi - stands for RNA interference. It is a specific, potent and highly successful approach for loss-of-functional studies in eukaryotic organisms. The process by which foreign, double-stranded RNA is recognized and degraded by specialized protein complex within many eukaryotic cells. It is believed to be an evolutionarily conserved defense mechanism against RNA viruses and transposable elements. 
rRNA - stands for ribosomal RNA, which is a permanent structural part of a ribosome.

RT-PCR - reverse transcriptase polymerase chain reaction. It is a highly sensitive technique for the detection and quantization of mRNA. This technique includes two parts: the synthesis of cDNA from mRNA by reverse transcript and the amplification of a specific cDNA by the polymerase chain reaction.

SARS - stands for severe acute respiratory syndrome, is a severe form of pneumonia which appeared in outbreaks in 2003. It is transmitted especially by contact with infectious material (as respiratory droplets or body fluids), and is characterized by fever, headache, body aches, a dry cough, hypoxia and usually pneumonia.

SEG - a computational program for masking or filtering low-complexity regions in amino acids.

siRNA - stands for silencing RNA, short interfering RNA or small interfering RNA. siRNA results in high levels of knockdown for transient knockdown experiments.

Smith-Waterman Algorithm - is a well-known algorithm for performing local sequence alignment in order to determine similar regions between two nucleotide or protein sequences. Instead of looking at the total sequence, the Smith-Waterman algorithm compares segments of all possible length and optimizes the similarity measure.

snRNA - stands for small nuclear RNA and is a small RNA molecule that functions in the nucleus by guiding the assembly of macromolecular complexes on the target RNA to allow site-specific modifications or processing reactions to occur.

spliceosome - a nucleoprotein particle that aids in the splicing of messenger RNA in eukaryotes. 
splice variant - an active mRNA that results from cutting and resealing by precise breakage of phosphodiester bonds at 5' and 3' splice sites (exon/intron junction). It also indicates a recombinant DNA molecule derived from cutting and resealing of DNA from different sources.

splice site - located in either 5' or 3' ends of the intron sequence that spliceosome complex will bind on the sequence to conduct the splicing events in order to get the coding region sequences united. It is also called exon/intron junction. The most common splice site in mammalian genomes is GT/AG.

start codon - the initiating signal of translation and the first amino acid in a polypeptide chain.

stop codon - the terminating signal in genomic sequence to notify the gene to stop translation. There are three common stop codons: TGA, TAG, and TAA.

synonymous mutation - a mutation that occurs in the nucleotide level but does not affects the amino acid in translation.

synteny - the occurrence of two or more genes on the same chromosome, whether or not they are linked.

thread - a portion of a program that can run independently of and concurrently with other portions of the program.

TIGR - short for The Institute for Genomic Research. It was a non-profit genomics research institute founded in 1992 by Craig Venter in Rockville, Maryland. It is now part of the J. Craig Venter Institute (http://www.tigr.org). 
transcription - the process that messenger RNA is synthesized from a DNA template resulting in the transfer of genetic information from the DNA molecule to the messenger RNA.

translation - the process by which messenger RNA directs the sequence of amino acids assembled by a ribosome during protein synthesis.

tRNA - stands for transfer RNA which is one of a class of RNA molecules that transport amino acids to ribosomes for incorporation into a polypeptide undergoing synthesis.

UCR - stands for upstream conserved region which are the regions being protected from harm or decay.

UniGene - an organized view of the transcriptome. UniGene provides sequence information in clusters that each UniGene entry is a set of transcript sequences that appear to come from the same transcription locus (gene or expressed pseudogene), together with information on protein similarities, gene expression, cDNA clone reagents and genomic location.

UTR - stands for untranslated region. It is a non-coding RNA that does not translated into a protein. It is located either in the $5^{\prime}$ end before the start codon of a gene or 3' end after the stop codon is recognized in gene regulation.

XNU - a computational program for masking or filtering low-complexity regions in amino acids. 


\title{
CURRICULUM VITAE
}

\author{
I. Elizabeth Cha
}

\section{CONTACT INFORMATION}

3F, 52, Lane 11, Kuang-Fu N. Rd., Taipei, Taiwan, R.O.C. 10594

Home: 886-2-2368-9651

Email: icha2886@yahoo.com

\section{DOB}

Taipei, Taiwan, R.O.C. - May 19, 1973

\section{EDUCATION \& TRAINING:}

Ph.D. (2003-Expected Summer 2008)

Computer Science and Engineering University of Louisville

Louisville, Kentucky, U.S.A.

Dissertation Title: "Computational Analysis of Expressed Sequence Tags for Understanding Gene Regulation” Advisor: Eric C. Rouchka

M.S., Computer Engineering and Computer Science University of Louisville Louisville, Kentucky, U.S.A.

2000-2002

B.S., Chemistry Chung Yuan Christian University Chung-Li, Tao-Yuan, Taiwan, R.O.C. 1991-1995

\section{TEACHING EXPERIENCE}

Substitute instructor, December 29, 2006 Information Structures (CECS302) - Graphs, Department of Computer Engineering and Computer Science, Speed School of Engineering, University of Louisville

Substitute instructor, February 23, 2006

Introduction to Bioinformatics (CECS660) - Search Sequence Database, Department of Computer Engineering and Computer Science, Speed School of Engineering, University of Louisville 
Co-Instructor, Spring 2006

Introduction to Computer Science and Engineering (CECS230), Department of Computer Engineering and Computer Science, Speed School of Engineering, University of Louisville

Co-Instructor, Fall 2003

Introduction to Computer Science and Engineering (CECS230), Department of Computer Engineering and Computer Science, Speed School of Engineering, University of Louisville

\section{TRAINING ON TEACHING}

Teachers on Teaching, University of Louisville, January 29, February 5, February 12, 2005

College Teaching, University of Louisville, Fall 2003

\section{PUBLICATIONS}

\section{Refereed Articles:}

I. E. Cha, C. M. Whitaker, X. Li, N. G. F. Cooper and E. C. Rouchka, "Detection of Novel Rat PDE4 Isoforms Using Computational Prediction and Experimental Verification", in preparation.

I. E. Cha, K. I. Hoblitzell and E. C. Rouchka, “Alternative Splicing Events”, University of Louisville Bioinformatics Technical Report Series, TR-ULBL-2007-03 (2007).

C. T. Chen, I Cha and S. J. Hsieh, "Thermal Stability of Robust Unsymmetrical Copperporphurins with Multiple Diphenyl and Nitro Substituents", Journal of the Chinese Chemical Society, 45: 741-748 (1998).

\section{Conference Proceedings:}

I. E. Cha and E. C. Rouchka, "Comparison of Current BLAST Software on Nucleotide Sequences", $4^{\text {th }}$ International Workshop on High Performance Computational Biology (HiCOMB 2005), $19^{\text {th }}$ International Parallel \& Distributed Symposium Conference (IPDPS 2005), Denver, Colorado, April 4-8, 2005, DOI: 10.1109/IPDPS.2005.145.

Poster Presentations:

I. E. Cha, C. M. Whitaker, E. C. Rouchka, X. Li and N. G. F. Cooper, "Computational Method for Splicing Pattern Recognition in Rat PDE4 Sequences", KRBIN SemiAnnual Review, December 4, 2007

I. E. Cha, C. M. Whitaker, E. C. Rouchka, X. Li and N. G. F. Cooper, "Computational Method for Splicing Pattern Recognition in Rat PDE4 Sequences", UT-ORNLKY Bioinformatics Summit 2007, April 14, 2007. 
I. E. Cha, C. M. Whitaker, E. C. Rouchka, X. Li and N. G. F. Cooper, "Computational Method for Splicing Pattern Recognition in Rat PDE4 Sequences", National Engineering Week 2007, eExpo07, University of Louisville, March 3, 2007.

I. E. Cha, C. M. Whitaker, E. C. Rouchka, X. Li and N. G. F. Cooper, “Automated Method for Splicing Pattern Recognition in Rat PDE4 Sequences", Poster GRD20, Research! Louisville 2006, October 10, 2006.

I. E. Cha, Y. Rudraraju, E. P. Stutzenberger and V. Akkineni, “The Sequence Alignment Problem - How to Get a Ligament from Alignment", National Engineering Week 2006, eExpo06, University of Louisville, March 4, 2006.

I. E. Cha and E. C. Rouchka, "Comparison of Current BLAST Software on Nucleotide Sequences”, Poster D-7, ISMB 2005, June 26, 2006.

I. E. Cha and E. C. Rouchka, "Comparison of Current BLAST Software on Nucleotide Sequences”, UT-ORNL-KBRIN Bioinformatics Summit 2005, April 2, 2005.

I. E. Cha and E. C. Rouchka, "Comparison of Current BLAST Software on Nucleotide Sequences", National Engineering Days 2005, University of Louisville, February 20-21, 2005.

E. C. Rouchka and I. E. Cha, "Bioinformatics", National Engineers Days 2005, University of Louisville, February 20-21, 2005.

E. C. Rouchka and I. E. Cha, "Bioinformatics", National Engineers Days 2004, University of Louisville, March 26-27, 2004.

\section{Invited Presentations:}

I. E. Cha, "In silico Prediction of Novel Tissue Specific Isoforms", Center for Computational Biology and Bioinformatics, Indiana University - Purdue University Indianapolis, Indianapolis, Indiana, May 9, 2008.

(Dr. Sean Mooney, Host)

I. E. Cha, "Computational Method for Recognition of Alternative Splicing", Genomic Research Center, Academia Sinica, Taipei, Taiwan, R.O.C., August 24, 2007. (Dr. C. Y. Lin, Host)

I. E. Cha, "Computational Method for Splice Pattern Recognition in Rat PDE4 Sequences", Section of Computer and Information Science, Kentucky Academy of Science (KAS 2006), Morehead University, Morehead, Kentucky, November $10,2006$.

I. E. Cha, "Pseudogene Detection and Characterization", Genomic Research Center, Academia Sinica, Taipei, Taiwan, R.O.C., December 23, 2005.

(Dr. C. Y. Lin, Host) 
I. E. Cha, "Comparison of Current BLAST Software on Nucleotide Sequences", Workshop on High Performance Computational Biology, HiCOMB 2005, Denver, Colorado, April 4, 2005.

\section{PROFESSIONAL ORGANIZATIONS \& SOCIETIES}

Association of Computing Machinery (ACM)

Institute of Electrical and Electronic Engineers (IEEE)

Upsilon Pi Epsilon Honor Society

Sigma Delta Epsilon, Graduate Women in Science

Toastmasters International at University of Louisville

\section{WORK EXPERIENCE}

Graduate Research Assistant

Bioinformatics Research Laboratory, Computer Engineering and Computer Science, University of Louisville

2005 - present

Student Grader

Engineering Analysis Core (ENCORE), University of Louisville

January 2002 - August 2002

Testing Assistant

ALSTOM Signaling Inc. (Formerly General Railway Signaling) - Taiwan June 1997 - July 2000

Assistant

ALSTOM Signaling Inc. (Formerly General Railway Signaling) - Taiwan October 1996 - June 1997

Research Assistant

Dr. C. T. Chen Laboratory, Institute of Chemistry, Academia Sinica August 1995 - July 1996

\section{HONORS \& AWARDS}

Raymond I. Fields Award, Computer Engineering and Computer Science Department, J. B. Speed School of Engineering Student Honors and Awards, University of Louisville, April 22, 2008.

First Place Graduate Research Poster Competition Award, National Engineering Week 2007, eExpo07, University of Louisville, March 3, 2007.

First Place Graduate Research Competition Award, Section of Computer and Information Science, $92^{\text {nd }}$ Annual Meeting of the Kentucky Academy of Science (KAS 2006), November 10, 2006. 
Graduate Scholarship (NT\$ 30,000), Mackay-HuiChen Lin Foundation, Taiwan, R.O.C., January 2005.

University Graduate Fellowship, University of Louisville, 2003 - 2004.

Future Professor Program, University of Louisville, August 2003 - May 2004. 